\title{
Broadening the scope of the zwitterionic 1,3-diaza- Claisen \\ rearrangement through a tethering strategy
}

Supporting Information

Matthew W. Luedtke, Joseph Pisano, Lucas Paquin, Joel Walker and José S. Madalengoitia*

Department of Chemistry, University of Vermont, Burlington, VT 05405

Jose.Madalengoitia@uvm.edu

Table of Contents

Computed Energies and Cartesian Coordinates.............................................. S2-S28

${ }^{1} \mathrm{H} /{ }^{13} \mathrm{C}$ NMR Spectra

S29-S128 


$\begin{array}{llll}\text { Ms-allyl-carbo } & & \\ \text { SCF energy }=-1026.559805 & & \\ \text { Free energy correction }=0.198242 & \\ \mathrm{C} & -2.74425600 & -2.54040300 & -0.95751700 \\ \mathrm{C} & -1.69650400 & -1.90756900 & -0.43738700 \\ \mathrm{C} & -1.72960900 & -1.15897100 & 0.86366600 \\ \mathrm{~N} & -1.33187000 & 0.23943400 & 0.69140000 \\ \mathrm{C} & -1.00898800 & 0.87070300 & 1.96181200 \\ \mathrm{C} & -2.29595400 & 0.99759500 & -0.08162300 \\ \mathrm{C} & -1.68403200 & 2.28835700 & -0.63467600 \\ \mathrm{~N} & -0.35923000 & 2.07381600 & -1.18722600 \\ \mathrm{C} & 0.33974300 & 1.09700900 & -1.15430700 \\ \mathrm{~N} & 1.17224500 & 0.17334600 & -1.28482700 \\ \mathrm{~S} & 1.96005300 & -0.26612000 & 0.13739200 \\ \mathrm{O} & 1.34853200 & -1.48559400 & 0.64490800 \\ \mathrm{O} & 2.09861500 & 0.88361500 & 1.02125800 \\ \mathrm{C} & 3.55397900 & -0.67571900 & -0.53716500 \\ \mathrm{H} & -2.67594200 & -3.09395600 & -1.88885300 \\ \mathrm{H} & -3.71102000 & -2.53741700 & -0.45790200 \\ \mathrm{H} & -0.73014300 & -1.91262800 & -0.94011200 \\ \mathrm{H} & -2.72929600 & -1.24189300 & 1.32990900 \\ \mathrm{H} & -0.99932800 & -1.61096100 & 1.54287100 \\ \mathrm{H} & -0.24166500 & 0.27958700 & 2.46696000 \\ \mathrm{H} & -1.89229700 & 0.96289300 & 2.61899500 \\ \mathrm{H} & -0.58564500 & 1.86494200 & 1.79243500 \\ \mathrm{H} & -2.61745600 & 0.37257600 & -0.92095300 \\ \mathrm{H} & -3.19638100 & 1.26126900 & 0.50456800 \\ \mathrm{H} & -1.58019300 & 3.05713900 & 0.13706100 \\ \mathrm{H} & -2.32122700 & 2.70639800 & -1.41848300 \\ \mathrm{H} & 3.98734000 & 0.22543400 & -0.97098800 \\ \mathrm{H} & 4.16126300 & -1.03867100 & 0.29370400 \\ \mathrm{H} & 3.41969500 & -1.45315700 & -1.28904900\end{array}$<smiles>C=CC[N+]1(C)CCN=C1N(C)C</smiles>

\section{Ms-allyl-zwitt}

SCF energy $=-1026.56581$

Free energy correction $=0.201567$

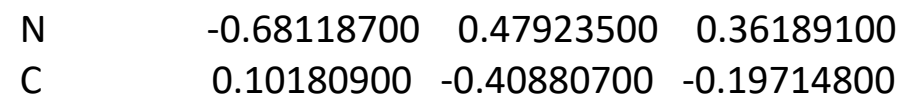




$\begin{array}{lrrr}\text { N } & 0.09036300 & -1.23848300 & -1.16711500 \\ \mathrm{C} & 1.29539000 & -2.03882800 & -1.18838200 \\ \mathrm{C} & 2.33407900 & -1.25340000 & -0.36574800 \\ \mathrm{~N} & 1.48647100 & -0.48777100 & 0.58464900 \\ \mathrm{C} & 1.20719800 & -1.25096000 & 1.83167800 \\ \mathrm{C} & 2.00506000 & 0.87876600 & 0.93102500 \\ \mathrm{C} & 2.12034500 & 1.77074200 & -0.26650600 \\ \mathrm{C} & 3.27945500 & 2.26910300 & -0.68680100 \\ \mathrm{~S} & -2.30516900 & 0.28847800 & 0.15973800 \\ \mathrm{O} & -2.69578900 & -1.07414500 & 0.51161800 \\ \mathrm{O} & -2.93704500 & 1.40900800 & 0.83928200 \\ \mathrm{C} & -2.58276100 & 0.48838000 & -1.59495800 \\ \mathrm{H} & 1.64811000 & -2.19246100 & -2.21176200 \\ \mathrm{H} & 1.11015700 & -3.03151900 & -0.75568500 \\ \mathrm{H} & 2.85980300 & -0.52908500 & -0.99007600 \\ \mathrm{H} & 3.05197400 & -1.86710600 & 0.18214900 \\ \mathrm{H} & 2.11463800 & -1.29333400 & 2.43843200 \\ \mathrm{H} & 0.87993400 & -2.25621600 & 1.55972100 \\ \mathrm{H} & 0.40052300 & -0.73809800 & 2.35669300 \\ \mathrm{H} & 2.97245200 & 0.73883300 & 1.42504600 \\ \mathrm{H} & 1.27787900 & 1.28807900 & 1.63665800 \\ \mathrm{H} & 1.18122500 & 2.02618300 & -0.75198400 \\ \mathrm{H} & 3.33111800 & 2.94971500 & -1.53024400 \\ \mathrm{H} & 4.21773800 & 2.02933800 & -0.19094200 \\ \mathrm{H} & -3.65396600 & 0.36382600 & -1.76313200 \\ \mathrm{H} & -2.01149500 & -0.27642200 & -2.12261300 \\ \mathrm{H} & -2.26769700 & 1.49286600 & -1.88018900\end{array}$<smiles></smiles>

\section{Ms-allyl-TS1}

SCF energy $=-1026.522025$

Free energy correction $=0.203558$

Imaginary frequency $=-502.87$

$\begin{array}{lrrr}\mathrm{C} & -2.50957600 & 0.62248700 & 1.02904900 \\ \mathrm{H} & -2.60612900 & 1.48616100 & 1.69499600 \\ \mathrm{H} & -3.49069800 & 0.38518500 & 0.60631300 \\ \mathrm{C} & -1.82168100 & -0.57961100 & 1.71289200 \\ \mathrm{H} & -1.68374400 & -0.39592700 & 2.78282800 \\ \mathrm{H} & -2.41040500 & -1.49470600 & 1.60439700 \\ \mathrm{C} & -2.02194200 & -0.18499900 & -1.59284300 \\ \mathrm{H} & -2.92330400 & 0.33994700 & -1.90646400 \\ \mathrm{H} & -1.10973000 & 0.10389300 & -2.11524200\end{array}$




$\begin{array}{llll}\mathrm{C} & -1.54646900 & 2.32926300 & -0.52926400 \\ \mathrm{H} & -1.25636700 & 3.00277000 & 0.28301900 \\ \mathrm{H} & -0.81165500 & 2.40555100 & -1.33011000 \\ \mathrm{H} & -2.54441400 & 2.59146200 & -0.88807200 \\ \mathrm{~N} & -1.56652400 & 0.94746100 & -0.04944200 \\ \mathrm{~N} & -0.51853000 & -0.73313500 & 1.06850400 \\ \mathrm{C} & -0.31586900 & 0.32896600 & 0.29385100 \\ \mathrm{~N} & 0.72364900 & 0.77600600 & -0.36534700 \\ \mathrm{~S} & 2.18483100 & 0.03094000 & -0.18420900 \\ \mathrm{O} & 3.15677100 & 0.87259300 & -0.86341000 \\ \mathrm{O} & 2.09889000 & -1.38386200 & -0.55262900 \\ \mathrm{C} & 2.53307500 & 0.09934600 & 1.56844700 \\ \mathrm{H} & 2.58045200 & 1.14621500 & 1.87025500 \\ \mathrm{H} & 1.75018600 & -0.44358300 & 2.09831000 \\ \mathrm{H} & 3.50288000 & -0.38088700 & 1.70903700 \\ \mathrm{C} & -2.11325100 & -1.48318900 & -1.06829800 \\ \mathrm{H} & -3.08262600 & -1.86613100 & -0.76046900 \\ \mathrm{C} & -0.94447800 & -2.04218300 & -0.57333900 \\ \mathrm{H} & -0.97244700 & -2.96233800 & 0.00379000 \\ \mathrm{H} & 0.02640300 & -1.79530300 & -0.99591700\end{array}$<smiles></smiles>

\begin{tabular}{lrcc}
\multicolumn{4}{l}{ SCF energy $=-1026.602294$} \\
Free energy correction $=0.200928$ \\
$\mathrm{~N}$ & -0.85177400 & 0.91385300 & 0.24559200 \\
$\mathrm{C}$ & 0.42614000 & 0.80502200 & 0.00534400 \\
$\mathrm{~N}$ & 1.19006300 & -0.16091100 & -0.59120900 \\
$\mathrm{C}$ & 2.60356000 & 0.18738600 & -0.46035900 \\
$\mathrm{C}$ & 2.54429800 & 1.69821300 & -0.26783100 \\
$\mathrm{~N}$ & 1.24692700 & 1.84814700 & 0.36547800 \\
$\mathrm{C}$ & 0.71265600 & 3.17081600 & 0.60017800 \\
$\mathrm{~S}$ & -1.96382900 & -0.23367700 & -0.09204300 \\
$\mathrm{O}$ & -2.02887800 & -0.51621100 & -1.52739700 \\
$\mathrm{O}$ & -1.84836800 & -1.37551500 & 0.81822300 \\
$\mathrm{C}$ & -3.43155300 & 0.66523000 & 0.35339800 \\
$\mathrm{C}$ & 0.88164400 & -1.58940300 & -0.72082900 \\
$\mathrm{C}$ & 1.09928500 & -2.33417300 & 0.56957700 \\
$\mathrm{C}$ & 2.12267100 & -3.16094600 & 0.76451900 \\
$\mathrm{H}$ & 3.15200700 & -0.11191200 & -1.35711300 \\
$\mathrm{H}$ & 3.03969700 & -0.31835800 & 0.41136900
\end{tabular}




$\begin{array}{lrrr}H & 2.57242600 & 2.22930000 & -1.23168200 \\ H & 3.34296200 & 2.08185300 & 0.37207500 \\ H & 1.43567000 & 3.74298500 & 1.18695200 \\ H & 0.51409500 & 3.70072900 & -0.34206500 \\ H & -0.21836300 & 3.07919800 & 1.15794900 \\ H & -3.37111000 & 0.93410000 & 1.40771100 \\ H & -3.50392800 & 1.55192800 & -0.27592600 \\ H & -4.27206700 & -0.00687300 & 0.17341900 \\ H & 1.55811900 & -1.96963300 & -1.49443000 \\ H & -0.13443000 & -1.70596000 & -1.09735600 \\ H & 0.36156000 & -2.15087000 & 1.34794900 \\ H & 2.26359900 & -3.68482500 & 1.70481000 \\ H & 2.85028300 & -3.35949800 & -0.02059700\end{array}$

\begin{tabular}{|c|c|c|c|}
\hline & TS2 & & \\
\hline \multicolumn{4}{|c|}{ SCF energy $=-1026.514212$} \\
\hline \multicolumn{4}{|c|}{ Free energy correction $=0.202433$} \\
\hline \multicolumn{4}{|c|}{ Imaginary frequency $=-505.59$} \\
\hline C & -2.69648100 & -1.20291800 & 0.12988100 \\
\hline $\mathrm{H}$ & -2.91680600 & -1.75367800 & 1.05118300 \\
\hline $\mathrm{H}$ & -3.63780800 & -0.84185900 & -0.29538600 \\
\hline C & -1.83538000 & -2.03536100 & -0.84686600 \\
\hline $\mathrm{H}$ & -1.90163900 & -3.10559000 & -0.63051900 \\
\hline $\mathrm{H}$ & -2.15877900 & -1.89600800 & -1.88523900 \\
\hline C & -2.14823300 & 1.40548500 & -0.74604300 \\
\hline $\mathrm{H}$ & -1.99360100 & 0.79368700 & -1.63694100 \\
\hline $\mathrm{H}$ & -3.19446600 & 1.56462000 & -0.48601900 \\
\hline C & -1.93367200 & 0.46512100 & 1.81252500 \\
\hline $\mathrm{H}$ & -1.73653300 & -0.32328400 & 2.54584000 \\
\hline $\mathrm{H}$ & -1.20462100 & 1.26357700 & 1.93942300 \\
\hline $\mathrm{H}$ & -2.94783300 & 0.85078000 & 1.94937600 \\
\hline $\mathrm{N}$ & -1.81426300 & -0.06784800 & 0.45382300 \\
\hline $\mathrm{N}$ & -0.46749400 & -1.55584400 & -0.69252300 \\
\hline C & -0.48447600 & -0.50631100 & 0.04383100 \\
\hline $\mathrm{N}$ & 0.49265400 & 0.35498000 & 0.43187800 \\
\hline$S$ & 2.06922100 & -0.03175300 & 0.02431000 \\
\hline $\mathrm{O}$ & 2.88730200 & 0.95221700 & 0.72175400 \\
\hline $\mathrm{O}$ & 2.22531700 & -0.17215400 & -1.41941700 \\
\hline C & 2.33528100 & -1.62732400 & 0.77651700 \\
\hline $\mathrm{H}$ & 2.18219900 & -1.53134300 & 1.85180500 \\
\hline $\mathrm{H}$ & 1.64645000 & -2.33915600 & 0.32198800 \\
\hline
\end{tabular}




$\begin{array}{lrrr}H & 3.37154600 & -1.89346600 & 0.55981500 \\ \mathrm{C} & -1.18532400 & 2.36990000 & -0.43329300 \\ \mathrm{H} & -1.39556700 & 3.11144800 & 0.33038100 \\ \mathrm{C} & 0.13016000 & 2.00355100 & -0.70933000 \\ \mathrm{H} & 0.96400100 & 2.58954700 & -0.33219600 \\ \mathrm{H} & 0.34743100 & 1.42639100 & -1.60673600\end{array}$<smiles>C=CCN(C)C1=NCCN1C</smiles>

\section{Ms-allyl-prod2}

SCF energy $=-1026.583696$

Free energy correction $=0.200249$

$\begin{array}{lrrr}\text { N } & 0.40915400 & 0.48194400 & -0.30578100 \\ \mathrm{C} & -0.74745100 & -0.19776600 & 0.17270100 \\ \mathrm{~N} & -1.16578600 & -0.09019400 & 1.37768400 \\ \mathrm{C} & -2.42123700 & -0.85457900 & 1.41050200 \\ \mathrm{C} & -2.40985000 & -1.71266400 & 0.12734600 \\ \mathrm{~N} & -1.49261800 & -0.94321200 & -0.70649100 \\ \mathrm{C} & -0.98668500 & -1.50934700 & -1.93833800 \\ \mathrm{~S} & 1.83902000 & -0.36389400 & -0.02021000 \\ \mathrm{O} & 1.54207600 & -1.75275100 & -0.32672300 \\ \mathrm{O} & 2.89362800 & 0.35864200 & -0.70633000 \\ \mathrm{C} & 2.11142300 & -0.23035700 & 1.74009100 \\ \mathrm{C} & 0.42938000 & 1.92767000 & 0.00809900 \\ \mathrm{C} & -0.67705000 & 2.60395300 & -0.74712500 \\ \mathrm{C} & -1.74733500 & 3.11506100 & -0.14841500 \\ \mathrm{H} & -3.26071500 & -0.14782200 & 1.40004500 \\ \mathrm{H} & -2.49185000 & -1.44982000 & 2.32327300 \\ \mathrm{H} & -3.39124600 & -1.81350000 & -0.34352200 \\ \mathrm{H} & -1.99639000 & -2.71800200 & 0.30592500 \\ \mathrm{H} & -1.81645300 & -1.63545100 & -2.63970800 \\ \mathrm{H} & -0.25586500 & -0.82426400 & -2.36944600 \\ \mathrm{H} & -0.49874500 & -2.47687300 & -1.76678600 \\ \mathrm{H} & 2.94797000 & -0.88848200 & 1.98097200 \\ \mathrm{H} & 2.36042500 & 0.80470400 & 1.97832400 \\ \mathrm{H} & 1.19619200 & -0.54089100 & 2.24719800 \\ \mathrm{H} & 0.30479900 & 2.08644600 & 1.08610900 \\ \mathrm{H} & 1.40615700 & 2.30087300 & -0.31319800 \\ \mathrm{H} & -0.57240100 & 2.62831300 & -1.82990000 \\ \mathrm{H} & -2.54202400 & 3.59561300 & -0.71011200 \\ \mathrm{H} & -1.86221200 & 3.06274500 & 0.93156300\end{array}$




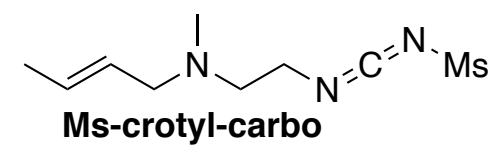

SCF energy $=-1065.867469$

Free energy correction $=0.224707$

C

$\begin{array}{lll}-3.29440900 & -1.31650600 & 0.08743700\end{array}$

C $\quad-2.02171900 \quad-1.02736100 \quad 0.35370700$

C $\quad-1.58061800-0.00952900 \quad 1.36420000$

$\begin{array}{lllll}\mathrm{N} & -0.76708600 & 1.04498100 & 0.75242500\end{array}$

$\begin{array}{llll}\text { C } & -0.03714100 & 1.81481500 & 1.74801800\end{array}$

C $\quad-1.54165200 \quad 1.88253300 \quad-0.14164000$

C $\quad-0.64233500 \quad 2.68104000-1.08957800$

$\mathrm{N} \quad 0.42442600 \quad 1.86860400 \quad-1.64591800$

C $\quad 0.75121800 \quad 0.74284800 \quad-1.38166700$

N $\quad \begin{array}{llll}\text { N } & 1.19361700 & -0.42334000 & -1.29527200\end{array}$

S $\quad 2.00876900 \quad-0.75673200 \quad 0.14057900$

$\begin{array}{lllll}\mathrm{O} & 1.12155600 & -1.51022800 & 1.01442800\end{array}$

$\begin{array}{lllll}0 & 2.65869900 & 0.44394000 & 0.64882700\end{array}$

C $\quad 3.24736600-1.86613200-0.48801000$

H $\quad-4.07794300 \quad-0.77591100 \quad 0.62215800$

$\mathrm{H} \quad-1.21438900-1.54810200-0.16168700$

H $\quad-2.45554000 \quad 0.41368200 \quad 1.89308200$

H $\quad-0.94409900 \quad-0.50293100 \quad 2.10566400$

$\begin{array}{llll}\mathrm{H} & 0.55098500 & 1.13171400 & 2.36543200\end{array}$

$\begin{array}{llll}H & -0.70941600 & 2.40621300 & 2.39538600\end{array}$

$\begin{array}{llll}\mathrm{H} & 0.67116400 & 2.48953700 & 1.25831900\end{array}$

$\begin{array}{llll}H & -2.18768000 & 1.22850800 & -0.73596500\end{array}$

$\begin{array}{llll}\mathrm{H} & -2.19272900 & 2.59300300 & 0.40256400\end{array}$

H $\quad-0.16205500 \quad 3.52514900-0.58564000$

$\begin{array}{llll}H & -1.22852000 & 3.09291600 & -1.91538700\end{array}$

$\mathrm{H} \quad 3.88337800-1.31803400-1.18306000$

H $\quad 3.82109600 \quad-2.21389200 \quad 0.37267100$

$\mathrm{H} \quad 2.74521700 \quad-2.69821600 \quad-0.98111400$

C $\quad-3.74951700 \quad-2.35648900-0.89284400$

$\mathrm{H} \quad-2.89704300-2.82912700 \quad-1.38797700$

$\mathrm{H} \quad-4.33201100-3.13937900-0.39396100$

H $\quad-4.39661600 \quad-1.92013300 \quad-1.66216500$<smiles>C/C=C\C[N+]1(C)CCN=C1[N+](C)(C)C</smiles>

Ms-crotyl-zwitt 
SCF energy $=-1065.874758$

Free energy correction $=0.227905$

C

$\mathrm{N}$

$\mathrm{C}$

0

C

$\mathrm{H}$

$\mathrm{H}$

$\mathrm{H}$

$\mathrm{H}$

$\mathrm{H}$

$\mathrm{H}$

$\mathrm{H}$

$\mathrm{H}$

$\mathrm{H}$

$\mathrm{H}$

$\mathrm{H}$

$\mathrm{H}$

$\mathrm{H}$

$\mathrm{H}$

C

$\mathrm{H}$

$\begin{array}{lll}-0.85363700 & -0.41450000 & -0.45851000\end{array}$

$\begin{array}{lllll}-0.24837100 & 0.48883000 & 0.27251500\end{array}$

$\begin{array}{llll}\mathrm{N} & -0.34022600 & 1.01345800 & 1.43217400\end{array}$

$\begin{array}{llll}\text { C } & 0.61072500 & 2.09116100 & 1.59744600\end{array}$

$\begin{array}{llll}C & 1.69326500 & 1.86122600 & 0.52580900\end{array}$

N $\quad \begin{array}{llll}0.94674600 & 1.15063000 & -0.54306800\end{array}$

$\begin{array}{llll}\text { C } & 0.30560900 & 2.08983000 & -1.50326300\end{array}$

C $\quad \begin{array}{llll}1.74226200 & 0.11810900 & -1.29398500\end{array}$

$2.25116600-0.97514000-0.40938800$

C $\quad 3.54874200-1.20155000-0.20523500$

$\begin{array}{llll}-2.45558300 & -0.65841500 & -0.17170200\end{array}$

$\begin{array}{lllll}0 & -3.15537400 & 0.62493800 & -0.16282900\end{array}$

$-2.88743500-1.69847300-1.09368100$

$\begin{array}{llll}-2.54643700 & -1.32882100 & 1.48302900\end{array}$

$\begin{array}{llll}H & 1.04769100 & 2.07972800 & 2.59968600\end{array}$

$\begin{array}{llll}0.11967700 & 3.06520700 & 1.46719600\end{array}$

$\begin{array}{llll}2.46453400 & 1.18025100 & 0.88957300\end{array}$

$\begin{array}{lllll}H & 2.15010500 & 2.76894900 & 0.12651200\end{array}$

$\begin{array}{lllll}1.07543400 & 2.54182900 & -2.13301900\end{array}$

$\begin{array}{llll}H & -0.22900300 & 2.85660200 & -0.93943400\end{array}$

$\begin{array}{llll}-0.40840400 & 1.51664700 & -2.09606300\end{array}$

$\begin{array}{llll}H & 2.55888300 & 0.64858200 & -1.79524400\end{array}$

$\begin{array}{llll}1.04677400 & -0.28529900 & -2.03417300\end{array}$

$\begin{array}{llll}H & 1.49102700 & -1.62061400 & 0.02769100\end{array}$

$\begin{array}{llll}H \quad 4.27949900 & -0.54074200 & -0.67609100\end{array}$

$\begin{array}{llll}H & -3.60197400 & -1.50428400 & 1.69796500\end{array}$

$\begin{array}{llll}-2.11978300 & -0.60060400 & 2.17369200\end{array}$

$\begin{array}{llll}H & -1.99373100 & -2.26886500 & 1.50227300\end{array}$

$\begin{array}{llll}4.09811200 & -2.32741600 & 0.61564100\end{array}$

H $\quad 3.29557000 \quad-2.91960400 \quad 1.06175500$

H $\quad 4.71483000-2.99008100-0.00105000$

H $\quad 4.74003000 \quad-1.94927500 \quad 1.41844500$<smiles></smiles>

Ms-crotyl-TS

SCF energy $=-1065.835181$

Free energy correction $=0.228628$

Imaginary frequency $=-455.74$

$\begin{array}{llll}\mathrm{C} & 2.35947500 & -1.08754700 & 1.04518100 \\ \mathrm{H} & 2.36646000 & -1.94039700 & 1.73293400\end{array}$

S8 


$\begin{array}{lrrr}\mathrm{H} & 3.36302700 & -0.96073100 & 0.62698100 \\ \mathrm{C} & 1.79088800 & 0.19241700 & 1.69594500 \\ \mathrm{H} & 1.65730200 & 0.06133100 & 2.77438200 \\ \mathrm{H} & 2.45843200 & 1.04813100 & 1.55015300 \\ \mathrm{C} & 1.98279700 & -0.19854200 & -1.62359000 \\ \mathrm{H} & 2.84758500 & -0.79670200 & -1.90514500 \\ \mathrm{H} & 1.05132800 & -0.44201700 & -2.13300500 \\ \mathrm{C} & 1.25659000 & -2.70379000 & -0.52230000 \\ \mathrm{H} & 0.92550500 & -3.36602700 & 0.28471700 \\ \mathrm{H} & 0.50653400 & -2.70959500 & -1.31305100 \\ \mathrm{H} & 2.22309600 & -3.04425400 & -0.90164900 \\ \mathrm{~N} & 1.39512600 & -1.33703500 & -0.03001700 \\ \mathrm{~N} & 0.49892100 & 0.43996600 & 1.06388200 \\ \mathrm{C} & 0.21534900 & -0.59473300 & 0.28351700 \\ \mathrm{~N} & -0.86273900 & -0.93055000 & -0.38973100 \\ \mathrm{~S} & -2.23433900 & -0.04359900 & -0.18629100 \\ \mathrm{O} & -3.28117200 & -0.73145400 & -0.92535600 \\ \mathrm{O} & -2.00386400 & 1.37824500 & -0.46521300 \\ \mathrm{C} & -2.61640300 & -0.17447000 & 1.55637300 \\ \mathrm{H} & -2.78441600 & -1.22507800 & 1.79514400 \\ \mathrm{H} & -1.78512700 & 0.24572900 & 2.12330100 \\ \mathrm{H} & -3.52782200 & 0.40316300 & 1.71981800 \\ \mathrm{C} & 2.15140000 & 1.08001400 & -1.09436500 \\ \mathrm{H} & 3.13867400 & 1.40992400 & -0.77733400 \\ \mathrm{C} & 1.01384500 & 1.74859800 & -0.65259200 \\ \mathrm{H} & 0.05115100 & 1.47833500 & -1.08189200 \\ \mathrm{C} & 1.04356300 & 3.06208000 & 0.05399500 \\ \mathrm{H} & 1.06879500 & 3.86566600 & -0.69271400 \\ \mathrm{H} & 0.13723400 & 3.17767800 & 0.64982900 \\ \mathrm{H} & 1.92778200 & 3.16726300 & 0.69081700\end{array}$<smiles>C=CCN(C)C(C)(C)CN=C=N</smiles>

\section{Ms-allyl-diMe1-carbo}

SCF Energy $=-1105.170606$

Free Energy Correction $=0.253193$

$\begin{array}{llll}\mathrm{C} & -0.78229200 & 3.78119900 & -0.35277500 \\ \mathrm{C} & -0.60479600 & 2.49066200 & -0.61515700 \\ \mathrm{C} & -1.67908600 & 1.45311300 & -0.44180300 \\ \mathrm{~N} & -1.19772400 & 0.29163500 & 0.31266800 \\ \mathrm{C} & -0.91104300 & 0.67291800 & 1.69445200\end{array}$




$\begin{array}{llrr}\text { C } & -2.03628100 & -0.91259200 & 0.16009900 \\ \mathrm{C} & -1.82972400 & -1.46618500 & -1.27159400 \\ \mathrm{~N} & -0.42552000 & -1.66492500 & -1.56595500 \\ \mathrm{C} & -1.58741900 & -1.99400700 & 1.15083500 \\ \mathrm{C} & -3.53881100 & -0.64865300 & 0.34560200 \\ \mathrm{C} & 0.51072700 & -0.94478000 & -1.34536100 \\ \mathrm{~N} & 1.58725600 & -0.31176400 & -1.29116900 \\ \mathrm{~S} & 2.26190100 & -0.16449300 & 0.24468500 \\ \mathrm{O} & 2.13319700 & 1.21864800 & 0.67081000 \\ \mathrm{O} & 1.78293000 & -1.23344300 & 1.11379800 \\ \mathrm{C} & 3.96710000 & -0.47312800 & -0.15689300 \\ \mathrm{H} & -1.72669400 & 4.15719000 & 0.03494800 \\ \mathrm{H} & 0.00724400 & 4.50809200 & -0.51268700 \\ \mathrm{H} & 0.35394800 & 2.12446900 & -0.97787300 \\ \mathrm{H} & -2.56278400 & 1.91679600 & 0.03236200 \\ \mathrm{H} & -1.99504800 & 1.11505200 & -1.43689100 \\ \mathrm{H} & -1.82736200 & 0.87261000 & 2.27766300 \\ \mathrm{H} & -0.30626200 & 1.58111300 & 1.67826500 \\ \mathrm{H} & -0.32174600 & -0.09894500 & 2.18942800 \\ \mathrm{H} & -2.25405900 & -0.79846000 & -2.02858300 \\ \mathrm{H} & -2.33893400 & -2.42805300 & -1.36633000 \\ \mathrm{H} & -0.50260400 & -2.12913500 & 1.12285100 \\ \mathrm{H} & -1.88066700 & -1.73737200 & 2.17215100 \\ \mathrm{H} & -2.07075000 & -2.94236400 & 0.89642700 \\ \mathrm{H} & -4.09944200 & -1.58802200 & 0.30322300 \\ \mathrm{H} & -3.93916500 & 0.01962400 & -0.42303200 \\ \mathrm{H} & -3.72402500 & -0.19382000 & 1.32418200 \\ \mathrm{H} & 4.53452000 & -0.34289300 & 0.76602600 \\ \mathrm{H} & 4.05783800 & -1.49363000 & -0.52856700 \\ \mathrm{H} & 4.27821700 & 0.25208300 & -0.90863800 \\ & & & \\ & & & \\ & & & \end{array}$<smiles>C=CCN1C(=NS(C)(=O)=O)[N-]CC1(C)C</smiles>

\section{Ms-allyl-diMe1-zwitt}

SCF energy $=-1105.175432$

Free energy correction $=0.256626$

$\begin{array}{llll}N & -1.22405200 & 0.67377700 & 0.18920800 \\ C & -0.43062900 & -0.36364100 & 0.13753000 \\ N & -0.48218300 & -1.62651300 & 0.32099400 \\ C & 0.77529100 & -2.24651100 & -0.02390900 \\ C & 1.85625800 & -1.14469000 & 0.08123300\end{array}$




$\begin{array}{lrrr}\mathrm{N} & 1.03647700 & 0.06511400 & -0.34819400 \\ \mathrm{~S} & -2.82826000 & 0.40145900 & -0.06300900 \\ \mathrm{O} & -3.48916900 & 1.69343200 & 0.04355900 \\ \mathrm{O} & -3.01067500 & -0.39520100 & -1.27472400 \\ \mathrm{C} & -3.35860600 & -0.59808500 & 1.31984700 \\ \mathrm{C} & 1.41314100 & 1.39674500 & 0.23277300 \\ \mathrm{C} & 2.76803300 & 1.89171700 & -0.17816700 \\ \mathrm{C} & 3.69360400 & 2.27549900 & 0.69708100 \\ \mathrm{C} & 0.88325600 & 0.18333500 & -1.82846500 \\ \mathrm{H} & 0.74175800 & -2.66996200 & -1.03962000 \\ \mathrm{H} & 1.00913500 & -3.07077100 & 0.65781400 \\ \mathrm{H} & -3.19283200 & -0.03075600 & 2.23637900 \\ \mathrm{H} & -4.42289200 & -0.79575600 & 1.18044800 \\ \mathrm{H} & -2.78249100 & -1.52397900 & 1.31310600 \\ \mathrm{H} & 1.33271800 & 1.31883500 & 1.31769700 \\ \mathrm{H} & 0.61516500 & 2.07032500 & -0.09317700 \\ \mathrm{H} & 2.96072300 & 2.01291400 & -1.24260100 \\ \mathrm{H} & 4.63977000 & 2.69773600 & 0.37490400 \\ \mathrm{H} & 3.52834000 & 2.19981700 & 1.76958100 \\ \mathrm{H} & 0.10808300 & 0.92992700 & -2.00692700 \\ \mathrm{H} & 1.82729900 & 0.47262200 & -2.28943700 \\ \mathrm{H} & 0.54664400 & -0.77428800 & -2.22788700 \\ \mathrm{C} & 3.07085200 & -1.39820100 & -0.80124300 \\ \mathrm{H} & 2.80071700 & -1.57892000 & -1.84441300 \\ \mathrm{H} & 3.57332100 & -2.29840600 & -0.43401100 \\ \mathrm{H} & 3.78581400 & -0.57132400 & -0.75074200 \\ \mathrm{C} & 2.27841300 & -0.99921200 & 1.54301600 \\ \mathrm{H} & 3.01167700 & -0.20159000 & 1.68148300 \\ \mathrm{H} & 2.74470100 & -1.93854500 & 1.85210600 \\ \mathrm{H} & 1.41389600 & -0.82545500 & 2.19052700\end{array}$

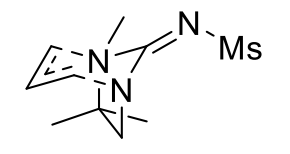

\section{Ms-allyl-diMe1-TS}

SCF energy $=-1105.135472$

Free energy correction $=0.257194$

Imaginary frequency $=-468.86$

$\begin{array}{llrr}C & 0.01418600 & -0.20771100 & -0.00225400 \\ C & -1.33732400 & 1.61778400 & 1.31856700 \\ C & -1.28083200 & 2.40391300 & 0.16073600 \\ C & -0.11089200 & 2.38107700 & -0.57229600 \\ \text { N } & -0.15420900 & 0.22492000 & -1.24399200 \\ N & -1.24231600 & -0.33493400 & 0.67429000\end{array}$




$\begin{array}{lrrr}\text { N } & 1.07469900 & -0.37467800 & 0.75670600 \\ \mathrm{~S} & 2.57497000 & -0.14509600 & 0.11977600 \\ \mathrm{O} & 2.72080500 & 1.20273500 & -0.43740700 \\ \mathrm{C} & -2.24864600 & -0.64278900 & -0.38415200 \\ \mathrm{C} & -1.55545400 & 0.02438600 & -1.60015700 \\ \mathrm{C} & -1.26630500 & -1.12104700 & 1.90754800 \\ \mathrm{H} & -0.93158300 & -2.14458100 & 1.72010400 \\ \mathrm{H} & -0.57635800 & -0.67114700 & 2.62077800 \\ \mathrm{H} & -2.28348900 & -1.12245900 & 2.30710800 \\ \mathrm{H} & -2.03664100 & 0.97612600 & -1.85234500 \\ \mathrm{H} & -1.62537200 & -0.62074500 & -2.48349200 \\ \mathrm{H} & -2.25597000 & 1.53946100 & 1.89608900 \\ \mathrm{H} & -0.41500500 & 1.44565200 & 1.87162800 \\ \mathrm{H} & -2.20100400 & 2.77589800 & -0.28133600 \\ \mathrm{C} & 2.67694800 & -1.29181600 & -1.24947900 \\ \mathrm{H} & 2.56224600 & -2.30364600 & -0.85926800 \\ \mathrm{H} & 1.89669200 & -1.04019400 & -1.96863100 \\ \mathrm{H} & 3.66690200 & -1.16409000 & -1.69053800 \\ \mathrm{O} & 3.52153000 & -0.57947500 & 1.13546300 \\ \mathrm{H} & 0.85107400 & 2.15554400 & -0.12020300 \\ \mathrm{H} & -0.06434700 & 2.82809600 & -1.56146400 \\ \mathrm{C} & -3.62762900 & -0.08217000 & -0.06128000 \\ \mathrm{H} & -4.31621400 & -0.34057800 & -0.87151500 \\ \mathrm{H} & -4.03078600 & -0.51426200 & 0.86142000 \\ \mathrm{H} & -3.61612500 & 1.00493300 & 0.03609600 \\ \mathrm{C} & -2.33462100 & -2.15874400 & -0.59345400 \\ \mathrm{H} & -2.76648300 & -2.66851700 & 0.27326400 \\ \mathrm{H} & -2.97572900 & -2.36404300 & -1.45574800 \\ \mathrm{H} & -1.34381500 & -2.58046200 & -0.79452000\end{array}$

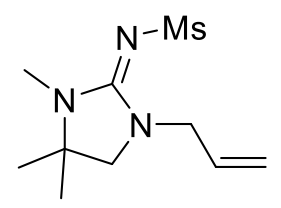

\section{Ms-allyl-diMe1-prod}

SCF energy $=-1105.219836$

Free energy correction $=0.254192$

$\begin{array}{lrrr}\text { N } & -0.86079400 & -1.24082100 & 0.33106100 \\ \mathrm{C} & 0.25857400 & -0.56545300 & 0.34914000 \\ \mathrm{~N} & 0.52628300 & 0.76812100 & 0.34004000 \\ \mathrm{C} & 1.96354200 & 0.98557800 & 0.40494500 \\ \mathrm{C} & 2.54268800 & -0.35727400 & -0.05810400 \\ \mathrm{~N} & 1.44831800 & -1.25301400 & 0.35505800 \\ \mathrm{C} & 3.84927900 & -0.69286800 & 0.64951500\end{array}$




$\begin{array}{lrrr}\text { C } & 2.71263700 & -0.37632200 & -1.58103400 \\ \mathrm{C} & 1.45390900 & -2.65646100 & -0.00615500 \\ \mathrm{C} & -0.33630000 & 1.87578700 & 0.74615600 \\ \mathrm{C} & -0.49182200 & 2.86529400 & -0.37473200 \\ \mathrm{C} & 0.00821900 & 4.09608000 & -0.32527700 \\ \mathrm{~S} & -2.30564000 & -0.55043400 & 0.00500400 \\ \mathrm{O} & -2.20014400 & 0.37502600 & -1.12710000 \\ \mathrm{O} & -2.96998900 & -0.06714200 & 1.21654700 \\ \mathrm{C} & -3.19114100 & -1.99342400 & -0.54223100 \\ \mathrm{H} & 2.26121200 & 1.21357900 & 1.44017900 \\ \mathrm{H} & 2.25731400 & 1.82012300 & -0.23966800 \\ \mathrm{H} & 3.69174600 & -0.76971100 & 1.72871800 \\ \mathrm{H} & 4.26650200 & -1.63727000 & 0.28651200 \\ \mathrm{H} & 4.58967100 & 0.08986200 & 0.45527100 \\ \mathrm{H} & 3.49420100 & 0.32840000 & -1.88213900 \\ \mathrm{H} & 1.77742700 & -0.09015800 & -2.07392200 \\ \mathrm{H} & 3.00366100 & -1.37194300 & -1.93042800 \\ \mathrm{H} & 0.74765500 & -3.18676600 & 0.63257400 \\ \mathrm{H} & 2.45605600 & -3.05761700 & 0.16038500 \\ \mathrm{H} & 1.16345700 & -2.81905700 & -1.05211100 \\ \mathrm{H} & 0.13793400 & 2.35949800 & 1.61220100 \\ \mathrm{H} & -1.30197600 & 1.49209400 & 1.07954600 \\ \mathrm{H} & -1.03785900 & 2.49793800 & -1.24018300 \\ \mathrm{H} & -0.11516900 & 4.79866600 & -1.14335300 \\ \mathrm{H} & 0.54763800 & 4.45344500 & 0.55006700 \\ \mathrm{H} & -2.70148900 & -2.39072700 & -1.43088200 \\ \mathrm{H} & -4.20705100 & -1.66755500 & -0.77087600 \\ \mathrm{H} & -3.19380100 & -2.72454700 & 0.26600200\end{array}$<smiles>C=CCN(C)CC(C)(C)N=C=N</smiles>

\section{Ms-ally-diMe2-carbo}

SCF energy $=-1105.171684$

Free energy correction $=0.25043$

$\begin{array}{llll}C & -0.02585400 & -4.05060600 & -0.60692800 \\ C & -0.28006600 & -2.75297900 & -0.73746800 \\ C & -1.45298600 & -2.06266900 & -0.10267600 \\ N & -1.04357900 & -0.84303500 & 0.59718000 \\ C & -0.27219500 & -1.18019100 & 1.79300300 \\ C & -2.15382800 & 0.04859200 & 0.88311600 \\ C & -2.29604700 & 1.19956500 & -0.13873900 \\ N & -1.01270900 & 1.91318700 & -0.17741400 \\ C & -2.60927600 & 0.70717100 & -1.55258300\end{array}$




$\begin{array}{llll}\mathrm{C} & -3.36685100 & 2.17438100 & 0.34150400 \\ \mathrm{C} & 0.06749300 & 1.49493900 & -0.50303500 \\ \mathrm{~N} & 1.20985700 & 1.26668500 & -0.94985800 \\ \mathrm{~S} & 2.32301000 & 0.52344300 & 0.06943800 \\ \mathrm{O} & 2.36543200 & -0.89720200 & -0.23713200 \\ \mathrm{O} & 2.11635200 & 0.96009500 & 1.44294300 \\ \mathrm{C} & 3.80630300 & 1.27830000 & -0.55571100 \\ \mathrm{H} & 0.82376300 & -4.51611300 & -1.09556800 \\ \mathrm{H} & -0.65981300 & -4.69499100 & -0.00175900 \\ \mathrm{H} & 0.38111600 & -2.11177600 & -1.31817900 \\ \mathrm{H} & -2.17449900 & -1.78609800 & -0.87929300 \\ \mathrm{H} & -1.97351100 & -2.75753500 & 0.58345600 \\ \mathrm{H} & 0.09007000 & -0.26648400 & 2.26791100 \\ \mathrm{H} & -0.87171000 & -1.75953200 & 2.51729300 \\ \mathrm{H} & 0.59829900 & -1.77191600 & 1.50134700 \\ \mathrm{H} & -3.11759800 & -0.48562300 & 0.93053500 \\ \mathrm{H} & -1.99584900 & 0.51122700 & 1.86318000 \\ \mathrm{H} & -2.70971600 & 1.55964800 & -2.23062100 \\ \mathrm{H} & -3.54967600 & 0.14610500 & -1.56016600 \\ \mathrm{H} & -1.80630900 & 0.06466500 & -1.92542600 \\ \mathrm{H} & -3.11313600 & 2.56947200 & 1.32906700 \\ \mathrm{H} & -4.33465800 & 1.66737900 & 0.40310200 \\ \mathrm{H} & -3.45775800 & 3.01363000 & -0.35312000 \\ \mathrm{H} & 3.89006400 & 1.04732300 & -1.61743100 \\ \mathrm{H} & 4.63593700 & 0.84214800 & 0.00294600 \\ \mathrm{H} & 3.74741800 & 2.35337500 & -0.38701700\end{array}$

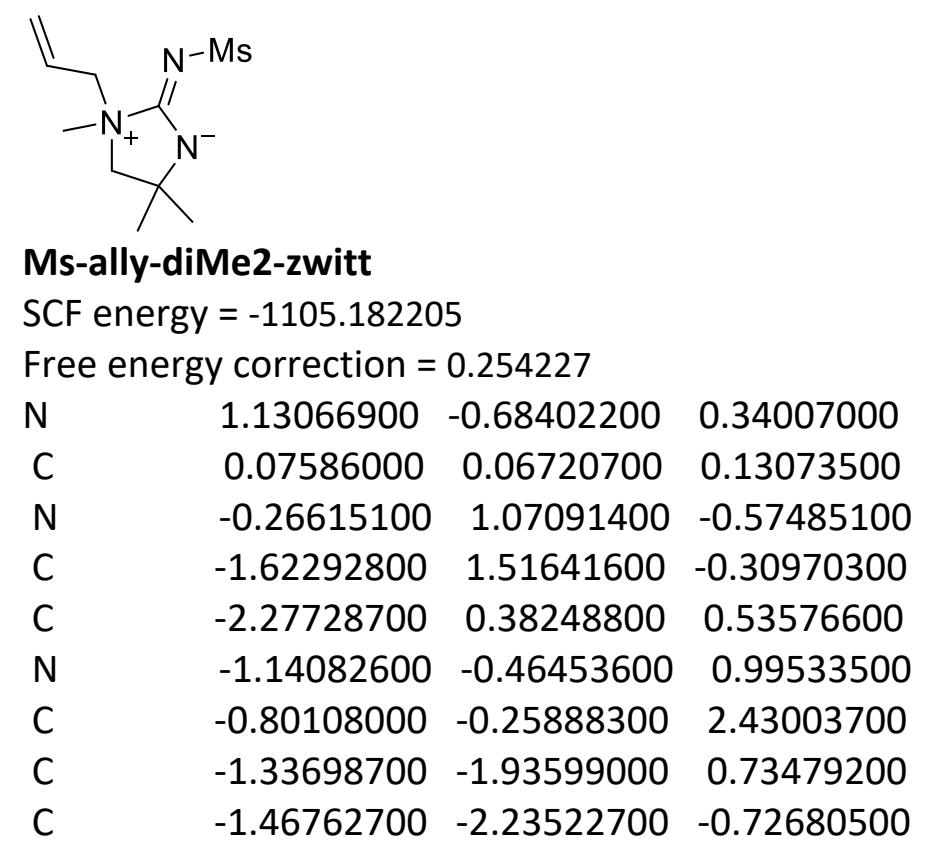




$\begin{array}{llcc}\text { C } & -2.56939800 & -2.74855900 & -1.26664800 \\ \mathrm{~S} & 2.59831900 & -0.04080800 & -0.03422300 \\ \mathrm{O} & 2.73055600 & 1.29486400 & 0.54046100 \\ \mathrm{O} & 3.58752400 & -1.05757800 & 0.29119900 \\ \mathrm{C} & 2.57437600 & 0.14411100 & -1.81302600 \\ \mathrm{H} & -2.93091700 & -0.23638200 & -0.08106200 \\ \mathrm{H} & -2.83132800 & 0.74799000 & 1.40315000 \\ \mathrm{H} & -1.60741100 & -0.66006700 & 3.04848200 \\ \mathrm{H} & -0.67874000 & 0.81029700 & 2.60730000 \\ \mathrm{H} & 0.14310900 & -0.76883600 & 2.62104700 \\ \mathrm{H} & -2.22625600 & -2.24481700 & 1.29386800 \\ \mathrm{H} & -0.44633300 & -2.41919300 & 1.14381700 \\ \mathrm{H} & -0.58223100 & -2.03697400 & -1.32717600 \\ \mathrm{H} & -3.45188500 & -2.96425000 & -0.66794900 \\ \mathrm{H} & -2.62320900 & -2.99098400 & -2.32292700 \\ \mathrm{H} & 3.53263500 & 0.57893100 & -2.10282400 \\ \mathrm{H} & 1.74962200 & 0.80729100 & -2.07749200 \\ \mathrm{H} & 2.45567100 & -0.84395100 & -2.25973500 \\ \mathrm{C} & -2.37484400 & 1.68954600 & -1.62849300 \\ \mathrm{H} & -2.37709000 & 0.74873300 & -2.18784300 \\ \mathrm{H} & -1.88037200 & 2.45158600 & -2.23628300 \\ \mathrm{H} & -3.41076500 & 2.00052300 & -1.45268400 \\ \mathrm{C} & -1.58597900 & 2.84278800 & 0.45464200 \\ \mathrm{H} & -2.59880800 & 3.20777500 & 0.65923600 \\ \mathrm{H} & -1.05549000 & 3.59008500 & -0.14049800 \\ \mathrm{H} & -1.04841000 & 2.73699700 & 1.40221200\end{array}$

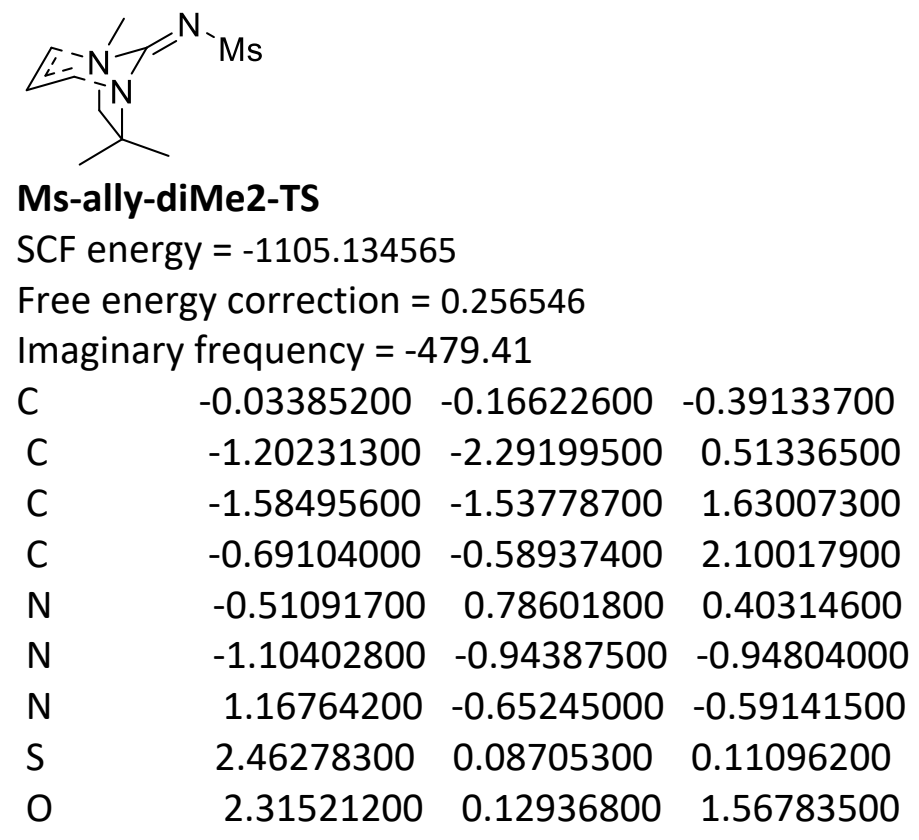




$\begin{array}{lrrr}\text { C } & -2.31969800 & -0.11756500 & -0.88110100 \\ \mathrm{C} & -1.85539100 & 1.13485200 & -0.08730000 \\ \mathrm{C} & -0.85146300 & -1.69495000 & -2.17829500 \\ \mathrm{H} & -0.75432300 & -1.00714700 & -3.02445300 \\ \mathrm{H} & 0.08181800 & -2.24424600 & -2.05731100 \\ \mathrm{H} & -1.68483000 & -2.37879800 & -2.35460500 \\ \mathrm{H} & -1.91440200 & -2.97501800 & 0.05230500 \\ \mathrm{H} & -0.15132700 & -2.54663400 & 0.37943900 \\ \mathrm{H} & -2.63708100 & -1.48340100 & 1.89583200 \\ \mathrm{C} & 2.41231900 & 1.77672500 & -0.47382700 \\ \mathrm{H} & 2.49791100 & 1.76849300 & -1.56101800 \\ \mathrm{H} & 1.47841300 & 2.22990800 & -0.14046200 \\ \mathrm{H} & 3.26991600 & 2.28388900 & -0.02862300 \\ \mathrm{O} & 3.64725200 & -0.54150300 & -0.45235800 \\ \mathrm{H} & 0.38411300 & -0.72063200 & 2.00315000 \\ \mathrm{H} & -0.99829000 & 0.14065900 & 2.84386200 \\ \mathrm{H} & -2.64888900 & 0.12845100 & -1.89614800 \\ \mathrm{H} & -3.11675900 & -0.67018300 & -0.37383700 \\ \mathrm{C} & -1.69701200 & 2.32453400 & -1.04492000 \\ \mathrm{H} & -2.66663400 & 2.63153900 & -1.45312300 \\ \mathrm{H} & -1.25545300 & 3.17220400 & -0.51322200 \\ \mathrm{H} & -1.03361200 & 2.06284700 & -1.87724200 \\ \mathrm{C} & -2.79730900 & 1.53484600 & 1.04356700 \\ \mathrm{H} & -3.02381000 & 0.70103800 & 1.71047500 \\ \mathrm{H} & -2.33492200 & 2.33305100 & 1.63165100 \\ \mathrm{H} & -3.74066300 & 1.91201200 & 0.63585800\end{array}$

$\begin{array}{llll} \\ \text { Free energy correction }=0.253039 \\ \mathrm{~N} & 0.97071800 & 0.65147600 & -0.09053600 \\ \mathrm{C} & 0.03503600 & -0.26476700 & -0.09990400 \\ \mathrm{~N} & -1.22524600 & 0.07994000 & -0.51181700 \\ \mathrm{C} & -2.19733900 & -0.96750100 & -0.16365400 \\ \mathrm{C} & -1.24545000 & -2.17444000 & -0.12101000 \\ \mathrm{~N} & 0.01959500 & -1.57321900 & 0.27061700 \\ \mathrm{C} & 1.13161800 & -2.40451800 & 0.69650100 \\ \mathrm{~S} & 2.56690900 & 0.29793300 & -0.02729700 \\ \mathrm{O} & 2.91379400 & -0.74144100 & -0.99934700\end{array}$




$\begin{array}{lrrr}\mathrm{O} & 3.03321900 & 0.12924000 & 1.35100400 \\ \mathrm{C} & 3.21923300 & 1.84482200 & -0.61630700 \\ \mathrm{C} & -1.57220400 & 1.46498000 & -0.77899100 \\ \mathrm{C} & -1.78765100 & 2.29783900 & 0.45806800 \\ \mathrm{C} & -2.85567800 & 3.06426400 & 0.65582200 \\ \mathrm{C} & -2.82018200 & -0.71965500 & 1.21454200 \\ \mathrm{C} & -3.27115500 & -1.12101100 & -1.23263700 \\ \mathrm{H} & -1.16170700 & -2.65553300 & -1.10702600 \\ \mathrm{H} & -1.55879200 & -2.92175000 & 0.61474200 \\ \mathrm{H} & 1.69041600 & -2.80803800 & -0.15261900 \\ \mathrm{H} & 1.81161200 & -1.82553700 & 1.32250800 \\ \mathrm{H} & 0.71598200 & -3.22079100 & 1.29363500 \\ \mathrm{H} & 2.86612700 & 2.00995000 & -1.63388100 \\ \mathrm{H} & 2.88610900 & 2.64034000 & 0.04990900 \\ \mathrm{H} & 4.30622700 & 1.75238600 & -0.59344200 \\ \mathrm{H} & -2.46479100 & 1.47875600 & -1.41301800 \\ \mathrm{H} & -0.74201500 & 1.88172100 & -1.35812000 \\ \mathrm{H} & -0.98195600 & 2.25750800 & 1.19009200 \\ \mathrm{H} & -3.66147300 & 3.11377400 & -0.07411400 \\ \mathrm{H} & -2.96302700 & 3.67747400 & 1.54490900 \\ \mathrm{H} & -3.49109500 & -1.54443400 & 1.47767700 \\ \mathrm{H} & -2.03837600 & -0.64662500 & 1.97777200 \\ \mathrm{H} & -3.39253200 & 0.21119100 & 1.21985300 \\ \mathrm{H} & -2.81989500 & -1.20824400 & -2.22507600 \\ \mathrm{H} & -3.95741600 & -0.26844100 & -1.22854400 \\ \mathrm{H} & -3.86288000 & -2.02047400 & -1.03418600\end{array}$<smiles>C/C=C/CN(C)CC(C)(C)N=C=NS(C)(=O)=O</smiles>

\section{Ms-crotyl-diMe2-carbo}

SCF energy $=-1144.479509$

Free energy correction $=0.27694$

$\begin{array}{llll}\mathrm{C} & -1.85064700 & 3.12728000 & -0.14193500 \\ \mathrm{C} & -0.86922600 & 2.28994000 & -0.46657900 \\ \mathrm{C} & 0.49796000 & 2.30081200 & 0.14932000 \\ \mathrm{~N} & 0.80249500 & 0.99638400 & 0.74703100 \\ \mathrm{C} & 0.06167400 & 0.82144900 & 1.99422200 \\ \mathrm{C} & 2.22046800 & 0.72832700 & 0.88843200 \\ \mathrm{C} & 2.79995800 & -0.11577900 & -0.27132000 \\ \mathrm{~N} & 1.98201500 & -1.32975900 & -0.38777500 \\ \mathrm{C} & 2.75992600 & 0.61202800 & -1.61659200 \\ \mathrm{C} & 4.22714300 & -0.53341000 & 0.06971700 \\ \mathrm{C} & 0.80442300 & -1.41716200 & -0.62215800\end{array}$




$\begin{array}{lrrr}\text { N } & -0.34819600 & -1.69209700 & -1.01429900 \\ \mathrm{~S} & -1.59525500 & -1.70347100 & 0.11496700 \\ \mathrm{O} & -2.35922500 & -0.47320600 & -0.01390500 \\ \mathrm{O} & -1.08982200 & -2.10764500 & 1.41941900 \\ \mathrm{C} & -2.55897400 & -3.03586200 & -0.56293400 \\ \mathrm{H} & -1.66038500 & 3.89530000 & 0.60996400 \\ \mathrm{H} & -1.05080100 & 1.49264700 & -1.18740700 \\ \mathrm{H} & 1.25322100 & 2.51246700 & -0.61515700 \\ \mathrm{H} & 0.57201200 & 3.10666700 & 0.90318000 \\ \mathrm{H} & 0.21636500 & -0.18842500 & 2.37851600 \\ \mathrm{H} & 0.36627700 & 1.55810900 & 2.75861400 \\ \mathrm{H} & -1.00536300 & 0.94155600 & 1.79260900 \\ \mathrm{H} & 2.81884400 & 1.65135400 & 0.96918900 \\ \mathrm{H} & 2.38327500 & 0.15854200 & 1.80948100 \\ \mathrm{H} & 3.19477900 & -0.02071700 & -2.39573300 \\ \mathrm{H} & 3.33651200 & 1.54152100 & -1.56293100 \\ \mathrm{H} & 1.73020400 & 0.84681900 & -1.90136500 \\ \mathrm{H} & 4.24821100 & -1.10909000 & 0.99914700 \\ \mathrm{H} & 4.85795900 & 0.35270600 & 0.19038100 \\ \mathrm{H} & 4.64546700 & -1.15162800 & -0.72900400 \\ \mathrm{H} & -2.82834600 & -2.78043600 & -1.58754700 \\ \mathrm{H} & -3.44925400 & -3.12464100 & 0.06170300 \\ \mathrm{H} & -1.96551700 & -3.94914700 & -0.52495600 \\ \mathrm{C} & -3.22971600 & 3.07968700 & -0.72757600 \\ \mathrm{H} & -3.98134400 & 2.95838600 & 0.05999600 \\ \mathrm{H} & -3.47048700 & 4.00539300 & -1.26288200 \\ \mathrm{H} & -3.32731100 & 2.23940800 & -1.41965600 \\ & & & \\ & & & \end{array}$

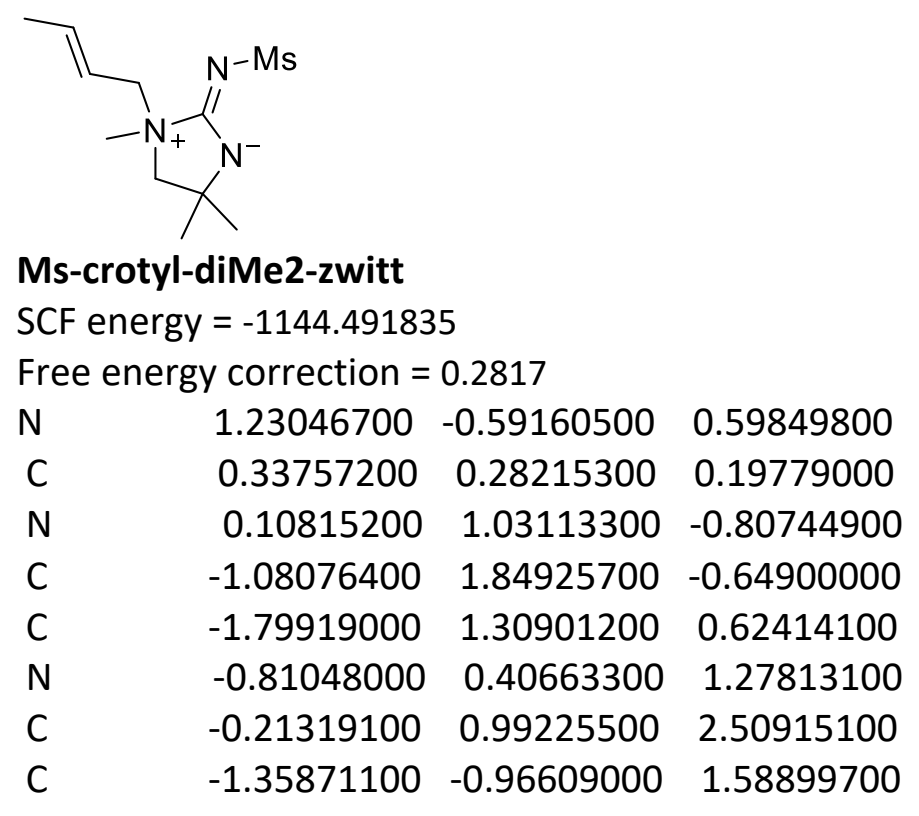




$\begin{array}{lrrr}\text { C } & -1.77057000 & -1.69787600 & 0.35154900 \\ \mathrm{C} & -3.03626500 & -2.00404400 & 0.06679800 \\ \mathrm{~S} & 2.70134900 & -0.56537100 & -0.13727200 \\ \mathrm{O} & 3.25489100 & 0.78593200 & -0.13146400 \\ \mathrm{O} & 3.47311900 & -1.65237800 & 0.44728000 \\ \mathrm{C} & 2.36695200 & -0.99628500 & -1.84064100 \\ \mathrm{H} & -2.67566400 & 0.71658300 & 0.35801900 \\ \mathrm{H} & -2.08934900 & 2.09474500 & 1.32501000 \\ \mathrm{H} & -0.97997200 & 1.04949700 & 3.28528700 \\ \mathrm{H} & 0.16351900 & 1.98781600 & 2.27028900 \\ \mathrm{H} & 0.61611500 & 0.35222300 & 2.80975400 \\ \mathrm{H} & -2.19762000 & -0.82222300 & 2.27802200 \\ \mathrm{H} & -0.54318100 & -1.48925400 & 2.09323000 \\ \mathrm{H} & -0.95969400 & -1.99955000 & -0.30988500 \\ \mathrm{H} & -3.82205400 & -1.69741900 & 0.76046500 \\ \mathrm{H} & 3.32192000 & -0.97385800 & -2.36857400 \\ \mathrm{H} & 1.67605900 & -0.25974300 & -2.25247300 \\ \mathrm{H} & 1.94471700 & -2.00194700 & -1.86407900 \\ \mathrm{C} & -1.98140300 & 1.68344700 & -1.87266000 \\ \mathrm{H} & -2.24850100 & 0.62927100 & -2.00128300 \\ \mathrm{H} & -1.45045500 & 2.01593600 & -2.76838800 \\ \mathrm{H} & -2.89951400 & 2.27299700 & -1.77073600 \\ \mathrm{C} & -0.67440800 & 3.31626600 & -0.48446300 \\ \mathrm{H} & -1.55489400 & 3.95936700 & -0.37384700 \\ \mathrm{H} & -0.11064100 & 3.63799600 & -1.36351200 \\ \mathrm{H} & -0.02682900 & 3.44744200 & 0.38857400 \\ \mathrm{C} & -3.47526300 & -2.76458200 & -1.14643400 \\ \mathrm{H} & -2.62164600 & -3.03812800 & -1.77129900 \\ \mathrm{H} & -4.00420600 & -3.68033200 & -0.86171900 \\ \mathrm{H} & -4.16930100 & -2.16810700 & -1.74866800 \\ & & & \\ & & & \end{array}$

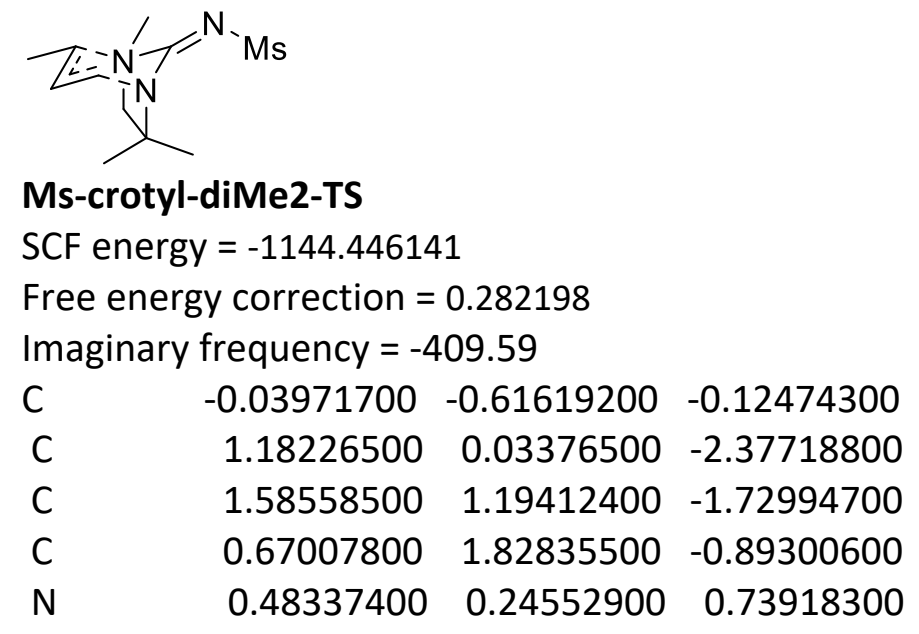




$\begin{array}{lccc}\mathrm{N} & 0.97704500 & -1.35118600 & -0.79610400 \\ \mathrm{~N} & -1.25931900 & -0.76743100 & -0.59848000 \\ \mathrm{~S} & -2.48546900 & 0.09331300 & 0.08038500 \\ \mathrm{O} & -2.22295300 & 1.53657000 & 0.03323800 \\ \mathrm{C} & 2.20137400 & -1.25383400 & 0.00317500 \\ \mathrm{C} & 1.79053500 & -0.29539400 & 1.15244900 \\ \mathrm{C} & 0.64392900 & -2.62382000 & -1.42605500 \\ \mathrm{H} & 0.52386500 & -3.40583200 & -0.66775200 \\ \mathrm{H} & -0.29693700 & -2.50227700 & -1.96277400 \\ \mathrm{H} & 1.44608500 & -2.90531800 & -2.11264100 \\ \mathrm{H} & 1.89015000 & -0.54178600 & -2.97069400 \\ \mathrm{H} & 0.12804300 & -0.12065900 & -2.59929800 \\ \mathrm{H} & 2.63925900 & 1.46209600 & -1.69510300 \\ \mathrm{C} & -2.48613500 & -0.37777300 & 1.80615200 \\ \mathrm{H} & -2.66397600 & -1.45180600 & 1.87064100 \\ \mathrm{H} & -1.52613200 & -0.09609600 & 2.24002500 \\ \mathrm{H} & -3.30126200 & 0.17185000 & 2.27988700 \\ \mathrm{O} & -3.71640300 & -0.40309400 & -0.51547100 \\ \mathrm{H} & -0.38832500 & 1.62586700 & -1.04118000 \\ \mathrm{H} & 2.48943200 & -2.24673500 & 0.36641500 \\ \mathrm{H} & 3.01744700 & -0.85176500 & -0.60777100 \\ \mathrm{C} & 1.56100200 & -1.09740200 & 2.44177000 \\ \mathrm{H} & 2.49804000 & -1.53555600 & 2.80405000 \\ \mathrm{H} & 1.15534300 & -0.44268100 & 3.21846300 \\ \mathrm{H} & 0.84056000 & -1.90483500 & 2.26742400 \\ \mathrm{C} & 2.81783000 & 0.79674700 & 1.42346500 \\ \mathrm{H} & 3.08785400 & 1.33663200 & 0.51415900 \\ \mathrm{H} & 2.41344100 & 1.51289000 & 2.14490900 \\ \mathrm{H} & 3.73005700 & 0.36186700 & 1.84503700 \\ \mathrm{C} & 0.94551900 & 3.06387200 & -0.10599300 \\ \mathrm{H} & 2.01044500 & 3.24380100 & 0.05650800 \\ \mathrm{H} & 0.52912900 & 3.91457500 & -0.66035900 \\ \mathrm{H} & 0.41856300 & 3.01188200 & 0.84910300\end{array}$<smiles>C=CC(C)N1C(=NS(=O)(=O)O)N(C)CC1(C)C</smiles>

\section{Ms-crotyl-diMe2-prod}

SCF energy $=-1144.522268$

Free energy correction $=0.281188$
$\mathrm{N}$
$\begin{array}{llll}0.99592000 & 0.53706700 & 0.07299200\end{array}$
C
$0.05573100-0.37090700-0.02380500$ 


$\begin{array}{lrrr}\text { N } & -1.21505100 & -0.00110200 & -0.38025800 \\ \mathrm{C} & -2.16305000 & -1.10659400 & -0.15752600 \\ \mathrm{C} & -1.19101000 & -2.29509400 & -0.22101600 \\ \mathrm{~N} & 0.06520900 & -1.71011600 & 0.21019900 \\ \mathrm{C} & 1.18398700 & -2.55679700 & 0.58268400 \\ \mathrm{~S} & 2.59188100 & 0.18623200 & 0.03341200 \\ \mathrm{O} & 2.89380600 & -0.77566600 & -1.02952400 \\ \mathrm{O} & 3.12755900 & -0.08033000 & 1.36999800 \\ \mathrm{C} & 3.21431400 & 1.77601700 & -0.47179200 \\ \mathrm{C} & -1.66275500 & 1.39525500 & -0.45467800 \\ \mathrm{C} & -1.48188400 & 2.14473400 & 0.84472400 \\ \mathrm{C} & -2.47962900 & 2.75848800 & 1.47312400 \\ \mathrm{C} & -2.81329800 & -0.99935400 & 1.22477800 \\ \mathrm{C} & -3.21367600 & -1.19344000 & -1.25934200 \\ \mathrm{H} & -1.10834200 & -2.69192300 & -1.24389000 \\ \mathrm{H} & -1.48798600 & -3.10755500 & 0.44999400 \\ \mathrm{H} & 1.74815200 & -2.89875200 & -0.28915300 \\ \mathrm{H} & 1.85810100 & -2.01420900 & 1.24671200 \\ \mathrm{H} & 0.77513800 & -3.41409600 & 1.12494600 \\ \mathrm{H} & 2.81874200 & 2.00880800 & -1.46057800 \\ \mathrm{H} & 2.90655000 & 2.52143300 & 0.26156100 \\ \mathrm{H} & 4.30174100 & 1.69051400 & -0.50115700 \\ \mathrm{H} & -0.46581700 & 2.18306200 & 1.23009000 \\ \mathrm{H} & -3.49859700 & 2.72904600 & 1.09027400 \\ \mathrm{H} & -3.49038700 & -1.84351000 & 1.39344800 \\ \mathrm{H} & -2.04711300 & -0.99789800 & 2.00682100 \\ \mathrm{H} & -3.38817200 & -0.07271300 & 1.31178700 \\ \mathrm{H} & -2.74162300 & -1.14653200 & -2.24489400 \\ \mathrm{H} & -3.95064200 & -0.38846700 & -1.18198200 \\ \mathrm{H} & -3.75529700 & -2.14139000 & -1.17434900 \\ \mathrm{H} & -2.31986000 & 3.31718700 & 2.38993800 \\ \mathrm{H} & -2.73900800 & 1.33414200 & -0.65338000 \\ \mathrm{C} & -1.01487300 & 2.12943400 & -1.63258700 \\ \mathrm{H} & 0.05330500 & 2.26498200 & -1.45823500 \\ \mathrm{H} & -1.15197100 & 1.55444600 & -2.55331800 \\ \mathrm{H} & -1.48360700 & 3.11028700 & -1.75094000\end{array}$

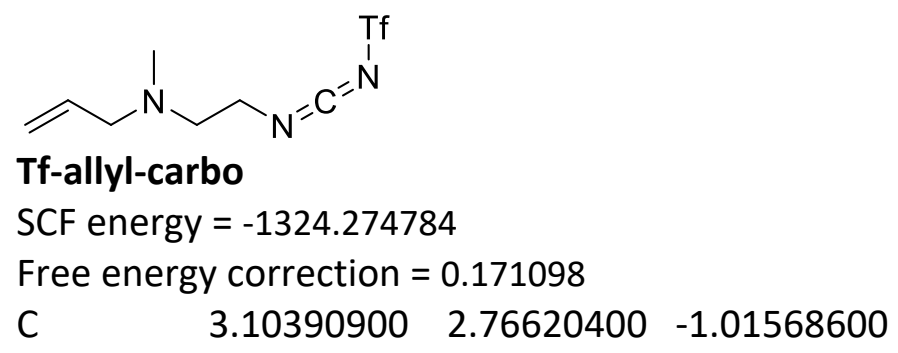




$\begin{array}{llcc}\mathrm{C} & 2.16237700 & 2.02544900 & -0.43834700 \\ \mathrm{C} & 2.36965400 & 1.24756100 & 0.82907700 \\ \mathrm{~N} & 2.09285100 & -0.18100200 & 0.64342600 \\ \mathrm{C} & 3.05494800 & -0.81154400 & -0.24269300 \\ \mathrm{C} & 1.98141100 & -0.87485600 & 1.91943800 \\ \mathrm{C} & 2.53908600 & -2.15163200 & -0.77698100 \\ \mathrm{~N} & 1.15289200 & -2.05993500 & -1.19787700 \\ \mathrm{C} & 0.36131300 & -1.16970500 & -1.09209400 \\ \mathrm{~N} & -0.56554200 & -0.32484400 & -1.13125900 \\ \mathrm{~S} & -1.21352700 & 0.04666500 & 0.35375000 \\ \mathrm{O} & -0.79786000 & 1.37493100 & 0.75734800 \\ \mathrm{O} & -1.13831200 & -1.06866600 & 1.27909200 \\ \mathrm{C} & -2.96993100 & 0.18778800 & -0.17668600 \\ \mathrm{~F} & -3.39176700 & -0.97653900 & -0.64210100 \\ \mathrm{~F} & -3.68813800 & 0.53213200 & 0.88129200 \\ \mathrm{~F} & -3.08486300 & 1.11227900 & -1.11501900 \\ \mathrm{H} & 2.90895300 & 3.33897600 & -1.91677100 \\ \mathrm{H} & 4.10469700 & 2.83765300 & -0.59487200 \\ \mathrm{H} & 1.16165900 & 1.96208100 & -0.86307300 \\ \mathrm{H} & 3.39146000 & 1.41228500 & 1.21784300 \\ \mathrm{H} & 1.66030600 & 1.60926000 & 1.58112900 \\ \mathrm{H} & 3.22495200 & -0.13740500 & -1.08783500 \\ \mathrm{H} & 4.02956100 & -0.98545300 & 0.24962400 \\ \mathrm{H} & 1.22905900 & -0.37638400 & 2.53445700 \\ \mathrm{H} & 2.94078700 & -0.89717300 & 2.46552900 \\ \mathrm{H} & 1.63716100 & -1.90119800 & 1.76301000 \\ \mathrm{H} & 2.58512400 & -2.94392900 & -0.02435400 \\ \mathrm{H} & 3.13697400 & -2.48146100 & -1.62996300\end{array}$

SCF energy =




$\begin{array}{lrrr}\text { O } & 1.01288700 & 1.54159400 & -0.85372700 \\ \mathrm{C} & 2.73669300 & -0.35711600 & -0.26209600 \\ \mathrm{~F} & 2.28384400 & -1.12961800 & -1.24121000 \\ \mathrm{~F} & 3.76383200 & 0.35553500 & -0.70937200 \\ \mathrm{~F} & 3.14972700 & -1.12860300 & 0.73848100 \\ \mathrm{C} & -2.01770200 & -1.42306200 & 1.95900400 \\ \mathrm{C} & -2.48591600 & 0.74635500 & 0.92693600 \\ \mathrm{C} & -2.43881900 & 1.57906900 & -0.31913400 \\ \mathrm{C} & -3.53349500 & 2.11412000 & -0.85287000 \\ \mathrm{H} & -2.09065100 & -2.21544500 & -2.21066000 \\ \mathrm{H} & -2.37759300 & -0.47145900 & -2.29652700 \\ \mathrm{H} & -3.05918900 & -2.39382500 & -0.04793400 \\ \mathrm{H} & -3.87006400 & -0.85829300 & -0.45314000 \\ \mathrm{H} & -1.67610700 & -2.43973700 & 1.75967400 \\ \mathrm{H} & -3.01456400 & -1.43577400 & 2.40590900 \\ \mathrm{H} & -1.29792800 & -0.91796900 & 2.60242500 \\ \mathrm{H} & -1.77323500 & 1.11665600 & 1.66877100 \\ \mathrm{H} & -3.49161000 & 0.71547600 & 1.35858100 \\ \mathrm{H} & -1.45120400 & 1.75439700 & -0.74864200 \\ \mathrm{H} & -3.48307100 & 2.74435200 & -1.73459500 \\ \mathrm{H} & -4.51778000 & 1.96102100 & -0.41416800\end{array}$

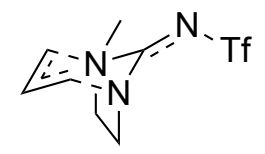

\section{Tf-allyl-TS}

SCF energy $=-1324.244643$

Free energy correction $=0.175512$

Imaginary frequency $=-498.44$

$\begin{array}{lrrr}\mathrm{C} & 3.19557000 & 0.69776300 & -0.95769200 \\ \mathrm{H} & 3.25808000 & 1.37437300 & -1.81568600 \\ \mathrm{H} & 4.10808200 & 0.78871200 & -0.36102000 \\ \mathrm{C} & 2.86101800 & -0.75289100 & -1.36993400 \\ \mathrm{H} & 2.88085400 & -0.86956800 & -2.45697400 \\ \mathrm{H} & 3.56815100 & -1.46810300 & -0.94165700 \\ \mathrm{C} & 2.38435200 & 0.47265200 & 1.70483500 \\ \mathrm{H} & 3.11583800 & 1.23336500 & 1.97403900 \\ \mathrm{H} & 1.35894800 & 0.68399200 & 2.00750200 \\ \mathrm{C} & 1.67620800 & 2.48603500 & -0.06746200 \\ \mathrm{H} & 1.42182400 & 2.85988700 & -1.06358400 \\ \mathrm{H} & 0.81018400 & 2.59359200 & 0.58528600 \\ \mathrm{H} & 2.52995800 & 3.03956800 & 0.32982300 \\ \mathrm{~N} & 2.02765500 & 1.06691300 & -0.14071300\end{array}$




$\begin{array}{llll}N & 1.50951600 & -1.01743900 & -0.88013600 \\ \mathrm{C} & 0.99999900 & 0.12575700 & -0.45736100 \\ \mathrm{~N} & -0.20952100 & 0.44343500 & -0.04163500 \\ \mathrm{~S} & -1.39337600 & -0.63087900 & -0.35151900 \\ \mathrm{O} & -1.36298600 & -1.76972600 & 0.56018700 \\ \mathrm{O} & -1.62970500 & -0.84649400 & -1.76845700 \\ \mathrm{C} & -2.78255400 & 0.41771000 & 0.23470500 \\ \mathrm{C} & 2.78326200 & -0.86699100 & 1.57411000 \\ \mathrm{H} & 3.84225400 & -1.10329900 & 1.51628000 \\ \mathrm{C} & 1.83756000 & -1.76818200 & 1.11386600 \\ \mathrm{H} & 2.12410700 & -2.77541100 & 0.82532100 \\ \mathrm{H} & 0.77839000 & -1.63181800 & 1.31890000 \\ \mathrm{~F} & -2.60846500 & 0.76487700 & 1.50526400 \\ \mathrm{~F} & -3.90472300 & -0.28323100 & 0.12738100 \\ \mathrm{~F} & -2.88538600 & 1.51580700 & -0.50384100\end{array}$

$\begin{array}{lrrr} & & \\ \text { Free energy correction }=0.173585 \\ \mathrm{~N} & -0.24110100 & 0.02216000 & 0.55011700 \\ \mathrm{C} & 0.84788300 & 0.67955900 & 0.22505800 \\ \mathrm{~N} & 2.03626200 & 0.02193500 & 0.10478600 \\ \mathrm{C} & 3.13814600 & 0.96981900 & 0.05987600 \\ \mathrm{C} & 2.42276800 & 2.23305000 & -0.42965900 \\ \mathrm{~N} & 1.05685200 & 2.00081200 & 0.02495700 \\ \mathrm{~S} & -1.71161000 & 0.47384300 & 0.07117800 \\ \mathrm{O} & -1.70861300 & 1.05655300 & -1.26692000 \\ \mathrm{O} & -2.49336900 & 1.10912000 & 1.12159200 \\ \mathrm{C} & -2.42035400 & -1.21056500 & -0.10632700 \\ \mathrm{~F} & -3.67425300 & -1.09624900 & -0.52527800 \\ \mathrm{~F} & -1.72723000 & -1.91307400 & -0.99533700 \\ \mathrm{~F} & -2.40392400 & -1.84530100 & 1.05748200 \\ \mathrm{C} & 0.06715500 & 3.06077700 & 0.03436300 \\ \mathrm{C} & 2.22793200 & -1.35514400 & 0.52187400 \\ \mathrm{C} & 3.17901600 & -2.06088400 & -0.40265800 \\ \mathrm{C} & 4.32384600 & -2.60587400 & -0.00477100 \\ \mathrm{H} & 3.92102500 & 0.62728100 & -0.61940000 \\ \mathrm{H} & 3.57167700 & 1.10777000 & 1.06131300 \\ \mathrm{H} & 2.45583900 & 2.32176700 & -1.52321100 \\ \mathrm{H} & 2.82121500 & 3.14893700 & 0.01320200\end{array}$




$\begin{array}{lrrr}H & -0.71335200 & 2.82953800 & 0.76293700 \\ H & 0.56842100 & 3.98053500 & 0.34707500 \\ H & -0.39498900 & 3.19854700 & -0.94670300 \\ H & 1.23862000 & -1.82217300 & 0.50353700 \\ H & 2.60452100 & -1.39179100 & 1.55501900 \\ H & 2.87670700 & -2.10502600 & -1.44805200 \\ H & 4.98115800 & -3.12421800 & -0.69534500 \\ H & 4.63699000 & -2.56520400 & 1.03605400\end{array}$<smiles>C/C=C/CN(C)CCN=C=N[Tl]</smiles>

\section{Tf-crotyl-carbo}

SCF energy $=-1363.582718$

Free energy correction $=0.197443$

$\begin{array}{lccc}\mathrm{C} & 3.39740600 & 2.10794700 & 0.05733100 \\ \mathrm{C} & 2.29445100 & 1.42715500 & 0.36504200 \\ \mathrm{C} & 2.25803800 & 0.27578500 & 1.32656300 \\ \mathrm{~N} & 1.76846300 & -0.95440000 & 0.69159500 \\ \mathrm{C} & 2.69177700 & -1.45484500 & -0.31023700 \\ \mathrm{C} & 1.42424800 & -1.96762400 & 1.67957800 \\ \mathrm{C} & 2.01063100 & -2.43833600 & -1.26730800 \\ \mathrm{~N} & 0.71138000 & -1.95211700 & -1.69440700 \\ \mathrm{C} & 0.07477000 & -1.00584700 & -1.33410100 \\ \mathrm{~N} & -0.69031000 & -0.03158300 & -1.13254700 \\ \mathrm{~S} & -1.41407400 & -0.03419800 & 0.36364300 \\ \mathrm{O} & -0.83665000 & 1.00200600 & 1.19578100 \\ \mathrm{O} & -1.60747200 & -1.37809900 & 0.87628200 \\ \mathrm{C} & -3.06643100 & 0.58112000 & -0.16304800 \\ \mathrm{~F} & -3.61468800 & -0.27540300 & -1.00946700 \\ \mathrm{~F} & -3.82431200 & 0.68690200 & 0.91775500 \\ \mathrm{~F} & -2.94491600 & 1.76445600 & -0.74066200 \\ \mathrm{H} & 4.34228900 & 1.80843000 & 0.51496300 \\ \mathrm{H} & 1.33451900 & 1.70308400 & -0.07199000 \\ \mathrm{H} & 3.25450600 & 0.12137900 & 1.78048800 \\ \mathrm{H} & 1.55519400 & 0.51037200 & 2.13302800 \\ \mathrm{H} & 3.05592200 & -0.59861800 & -0.88631100 \\ \mathrm{H} & 3.56901100 & -1.95664200 & 0.13852000 \\ \mathrm{H} & 0.70636700 & -1.54860100 & 2.38811200 \\ \mathrm{H} & 2.31021900 & -2.32907100 & 2.23068800 \\ \mathrm{H} & 0.93560400 & -2.81795000 & 1.19513900 \\ \mathrm{H} & 1.84199400 & -3.41637100 & -0.80743900 \\ \mathrm{H} & 2.62662000 & -2.60098700 & -2.15500400 \\ \mathrm{C} & 3.44535500 & 3.28653100 & -0.86854000\end{array}$




$\begin{array}{llll}H & 2.45708600 & 3.50025300 & -1.28393600 \\ H & 3.79753200 & 4.18237600 & -0.34512000 \\ H & 4.13833000 & 3.11003600 & -1.69872000\end{array}$<smiles>C/C=C/C[N+]1(C)CC[N-]/C1=N/[Tl]</smiles>

\section{Tf-crotyl-zwitt}

SCF energy $=-1363.598485$

Free energy correction $=0.202105$

$\begin{array}{lrrr}\text { N } & -0.56574000 & -0.58488100 & -0.75407400 \\ \mathrm{C} & 0.32093900 & -0.81590400 & 0.20454400 \\ \mathrm{~N} & 0.42539300 & -0.67144600 & 1.45728800 \\ \mathrm{C} & 1.75848700 & -0.98418900 & 1.92265300 \\ \mathrm{C} & 2.45865000 & -1.74519100 & 0.77576300 \\ \mathrm{~N} & 1.64458100 & -1.40671400 & -0.42693200 \\ \mathrm{~S} & -1.54964900 & 0.68386500 & -0.60330800 \\ \mathrm{O} & -2.14871000 & 0.95208400 & -1.89653400 \\ \mathrm{O} & -0.95390400 & 1.77071400 & 0.16556800 \\ \mathrm{C} & -2.93998200 & 0.05965200 & 0.42960800 \\ \mathrm{~F} & -2.50780800 & -0.30568900 & 1.62986000 \\ \mathrm{~F} & -3.84755900 & 1.01970800 & 0.56714100 \\ \mathrm{~F} & -3.50680000 & -0.98914100 & -0.15950100 \\ \mathrm{C} & 1.32852600 & -2.58181900 & -1.27883600 \\ \mathrm{C} & 2.25940000 & -0.31479100 & -1.27701600 \\ \mathrm{C} & 2.45758600 & 0.95134700 & -0.50431400 \\ \mathrm{C} & 3.66714200 & 1.43352800 & -0.21733200 \\ \mathrm{H} & 1.71682400 & -1.59163700 & 2.83062800 \\ \mathrm{H} & 2.29210100 & -0.05752600 & 2.16060700 \\ \mathrm{H} & 2.40592400 & -2.82545700 & 0.92032500 \\ \mathrm{H} & 3.49610200 & -1.44761700 & 0.61508200 \\ \mathrm{H} & 0.84764200 & -3.33966500 & -0.65881000 \\ \mathrm{H} & 2.25581800 & -2.97000400 & -1.70636400 \\ \mathrm{H} & 0.63658000 & -2.25459600 & -2.05428700 \\ \mathrm{H} & 1.55137400 & -0.16868900 & -2.09683000 \\ \mathrm{H} & 3.20294800 & -0.71202600 & -1.66486100 \\ \mathrm{H} & 1.55338300 & 1.48323300 & -0.20111100 \\ \mathrm{H} & 4.54926700 & 0.88197300 & -0.55082200 \\ \mathrm{C} & 3.91659600 & 2.70959200 & 0.52541600 \\ \mathrm{H} & 4.48638700 & 3.41230500 & -0.09197100 \\ \mathrm{H} & 2.97735800 & 3.18531100 & 0.81670500 \\ \mathrm{H} & 4.50939500 & 2.52422700 & 1.42797500\end{array}$




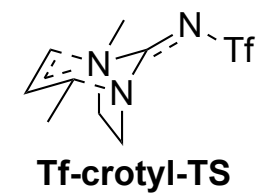

SCF energy $=-1363.558334$

Free energy correction $=0.201411$

Imaginary frequency $=-448.03$

$\begin{array}{lccc}\mathrm{C} & -2.99946200 & -1.31344100 & -0.83384000 \\ \mathrm{H} & -3.02386400 & -2.11232300 & -1.58265400 \\ \mathrm{H} & -3.89546600 & -1.38270400 & -0.20938800 \\ \mathrm{C} & -2.78412500 & 0.08075100 & -1.46441700 \\ \mathrm{H} & -2.85095100 & 0.03829000 & -2.55526600 \\ \mathrm{H} & -3.52932300 & 0.80235800 & -1.11438900 \\ \mathrm{C} & -2.19738600 & -0.55138600 & 1.78411500 \\ \mathrm{H} & -2.87950500 & -1.30585700 & 2.17163200 \\ \mathrm{H} & -1.15330600 & -0.66206100 & 2.07331900 \\ \mathrm{C} & -1.34360500 & -2.81787500 & 0.30347800 \\ \mathrm{H} & -1.09027900 & -3.34990200 & -0.61919600 \\ \mathrm{H} & -0.45454000 & -2.75153800 & 0.93029700 \\ \mathrm{H} & -2.14275800 & -3.34871300 & 0.82583500 \\ \mathrm{~N} & -1.79755300 & -1.46423900 & -0.00372800 \\ \mathrm{~N} & -1.44327400 & 0.50443800 & -1.07401600 \\ \mathrm{C} & -0.85448500 & -0.50846900 & -0.47042200 \\ \mathrm{~N} & 0.38433800 & -0.65212800 & -0.03018000 \\ \mathrm{~S} & 1.45924200 & 0.45128400 & -0.54806000 \\ \mathrm{O} & 1.24039100 & 1.77834400 & 0.02298600 \\ \mathrm{O} & 1.78709300 & 0.32960600 & -1.95898900 \\ \mathrm{C} & 2.90170700 & -0.22609000 & 0.36406300 \\ \mathrm{C} & -2.67210000 & 0.71044700 & 1.42668400 \\ \mathrm{H} & -3.74368300 & 0.88179100 & 1.34631500 \\ \mathrm{C} & -1.78031900 & 1.60170200 & 0.84061300 \\ \mathrm{H} & -0.71700100 & 1.48102200 & 1.03941200 \\ \mathrm{C} & -2.17471400 & 2.92800800 & 0.28449800 \\ \mathrm{H} & -2.12626900 & 3.67612100 & 1.08576800 \\ \mathrm{H} & -1.47353300 & 3.22202500 & -0.49801100 \\ \mathrm{H} & -3.19552800 & 2.92169900 & -0.10984600 \\ \mathrm{~F} & 3.95125300 & 0.54862500 & 0.11887400 \\ \mathrm{~F} & 3.17314100 & -1.46271400 & -0.03395800 \\ \mathrm{~F} & 2.66789700 & -0.23399200 & 1.67286200\end{array}$




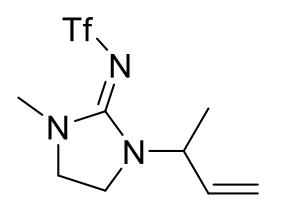

\section{Tf-crotyl-prod}

SCF energy $=-1363.63124$

Free energy correction $=0.201646$

$\begin{array}{llcc}\text { N } & 0.50221700 & 0.39498900 & 0.91969400 \\ \mathrm{C} & -0.49958700 & 0.99946900 & 0.30070400 \\ \mathrm{~N} & -1.67723300 & 0.37739100 & 0.07048800 \\ \mathrm{C} & -2.69416800 & 1.33393100 & -0.34622500 \\ \mathrm{C} & -1.82919500 & 2.51586600 & -0.81361900 \\ \mathrm{~N} & -0.58371800 & 2.28488400 & -0.09241600 \\ \mathrm{~S} & 1.95582200 & 0.33005300 & 0.25523700 \\ \mathrm{O} & 2.99855100 & 0.42063600 & 1.25714200 \\ \mathrm{O} & 2.06702500 & 1.08402400 & -0.99048700 \\ \mathrm{C} & 1.93737500 & -1.42671000 & -0.29024300 \\ \mathrm{~F} & 3.06236800 & -1.72556900 & -0.92378500 \\ \mathrm{~F} & 1.78699900 & -2.24302800 & 0.74622100 \\ \mathrm{~F} & 0.90787900 & -1.61474100 & -1.12500100 \\ \mathrm{C} & 0.45829700 & 3.28416000 & 0.01209400 \\ \mathrm{C} & -1.97171400 & -0.99338900 & 0.48305900 \\ \mathrm{C} & -2.57153400 & -1.72529400 & -0.69262900 \\ \mathrm{C} & -3.75344300 & -2.33322800 & -0.70645200 \\ \mathrm{C} & -2.81257600 & -1.01422800 & 1.75548600 \\ \mathrm{H} & -3.33517700 & 1.61282800 & 0.50048400 \\ \mathrm{H} & -3.31718800 & 0.91572700 & -1.13970100 \\ \mathrm{H} & -2.25469100 & 3.48547100 & -0.54339200 \\ \mathrm{H} & -1.65260500 & 2.49362900 & -1.89641700 \\ \mathrm{H} & 1.12707700 & 3.00913400 & 0.83051100 \\ \mathrm{H} & 1.04195200 & 3.35800300 & -0.90824500 \\ \mathrm{H} & -0.01077600 & 4.24487600 & 0.24388000 \\ \mathrm{H} & -0.99116300 & -1.43143300 & 0.69901100 \\ \mathrm{H} & -1.94375800 & -1.72782000 & -1.58294300 \\ \mathrm{H} & -4.40279800 & -2.35614300 & 0.16422100 \\ \mathrm{H} & -4.10874900 & -2.84867500 & -1.59285000 \\ \mathrm{H} & -2.96780200 & -2.04320900 & 2.09040600 \\ \mathrm{H} & -3.79483500 & -0.55648400 & 1.59716300 \\ \mathrm{H} & -2.29204300 & -0.46830000 & 2.54651000\end{array}$


<smiles>C=CCN(CC=C)CCN1C(=O)c2ccccc2C1=O</smiles>

$11 \mathrm{a}$

${ }^{1} \mathrm{H} ; \mathrm{CDCl}_{3} ; 500 \mathrm{MHz}$

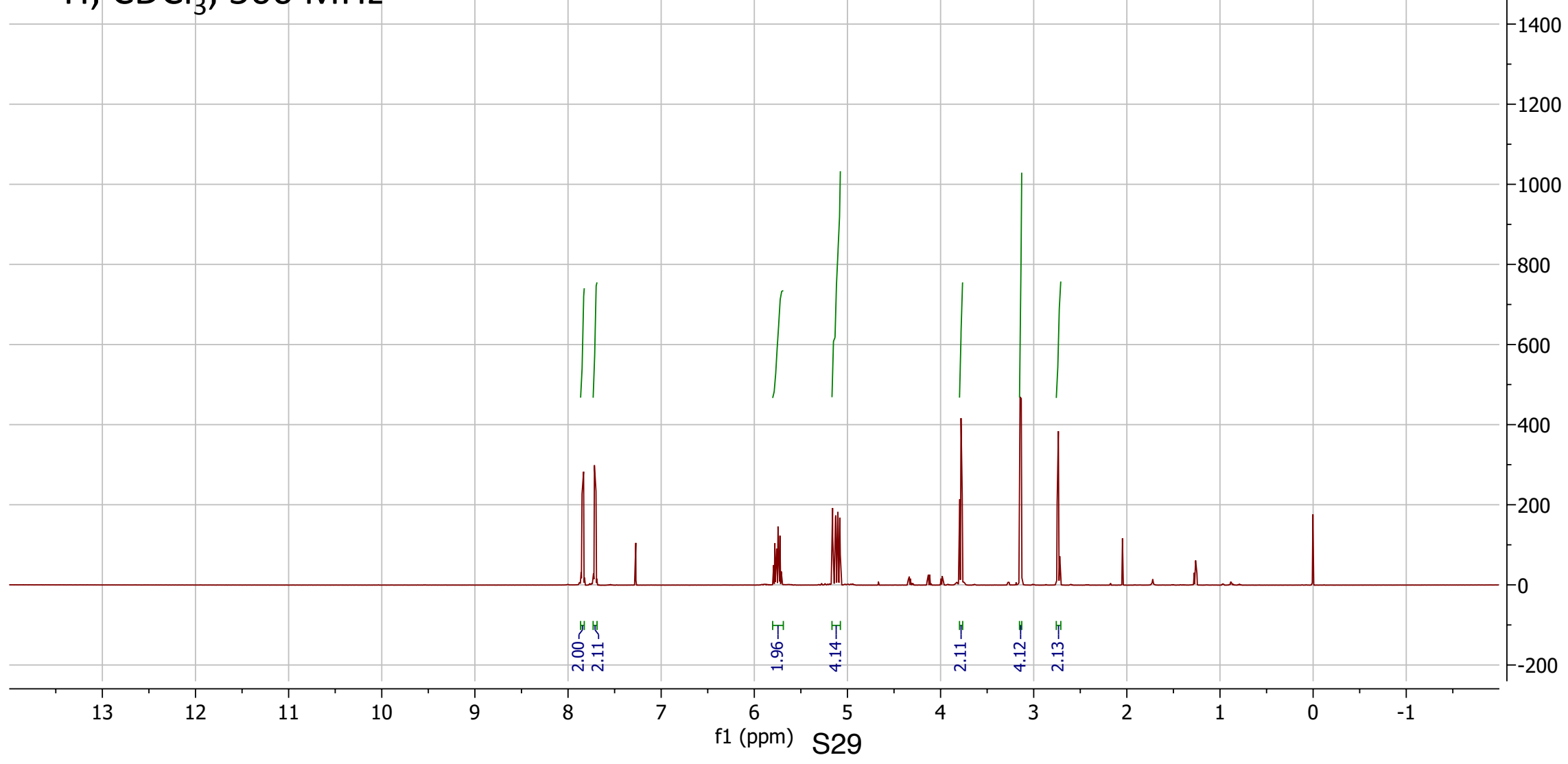



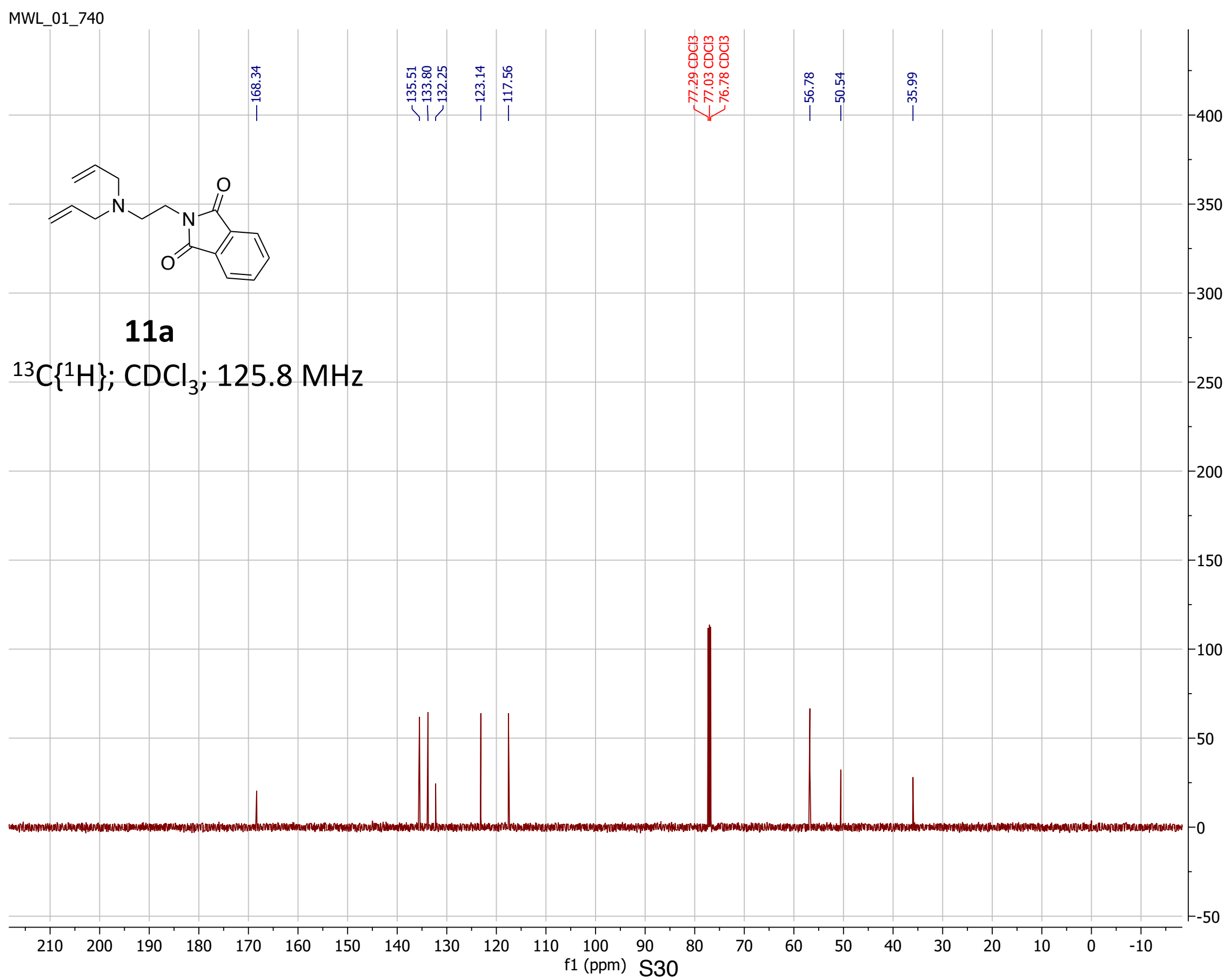


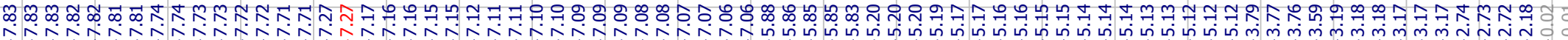

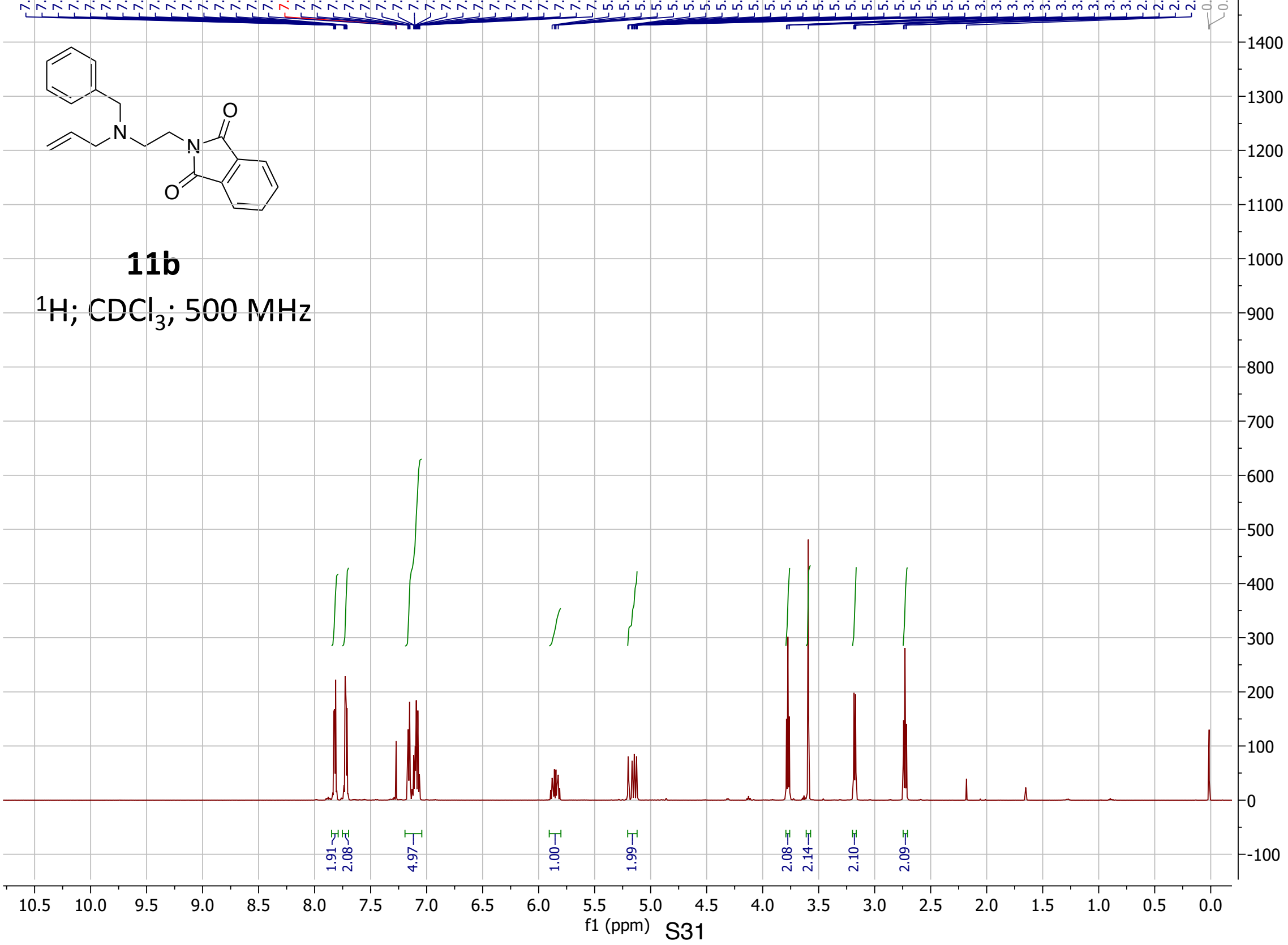


<smiles>C=CCN(CCc1ccccc1)CCN1C(=O)c2ccccc2C1=O</smiles>

\section{1c}

\section{${ }^{1} \mathrm{H} ; \mathrm{CDCl}_{3} ; 500 \mathrm{MHz}$}

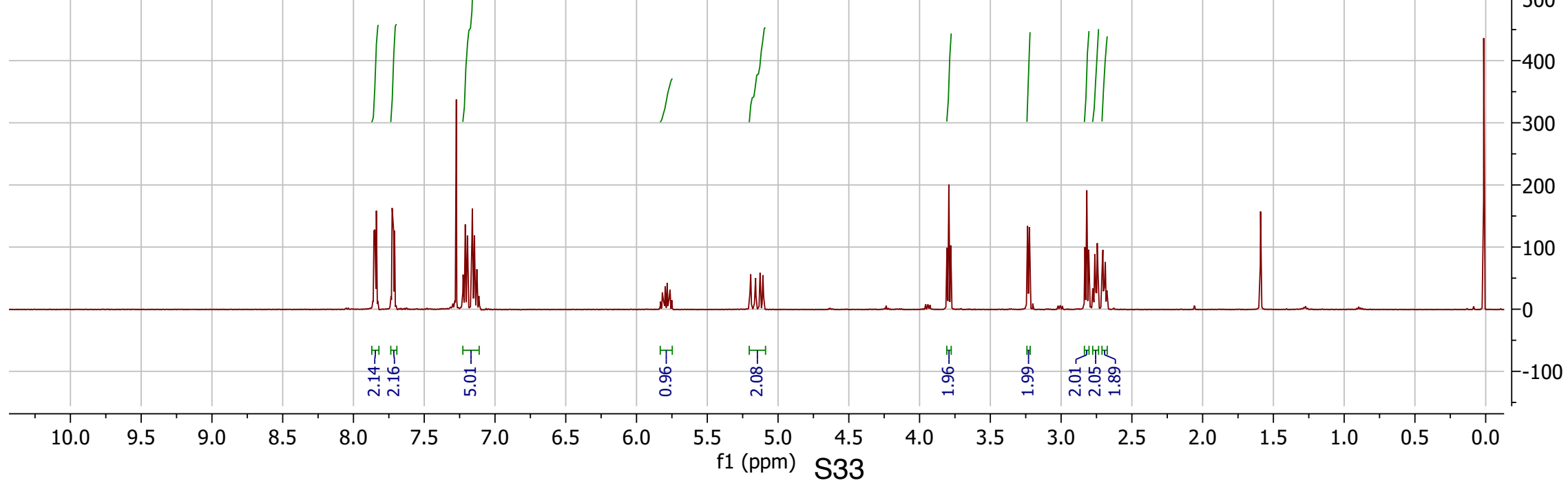




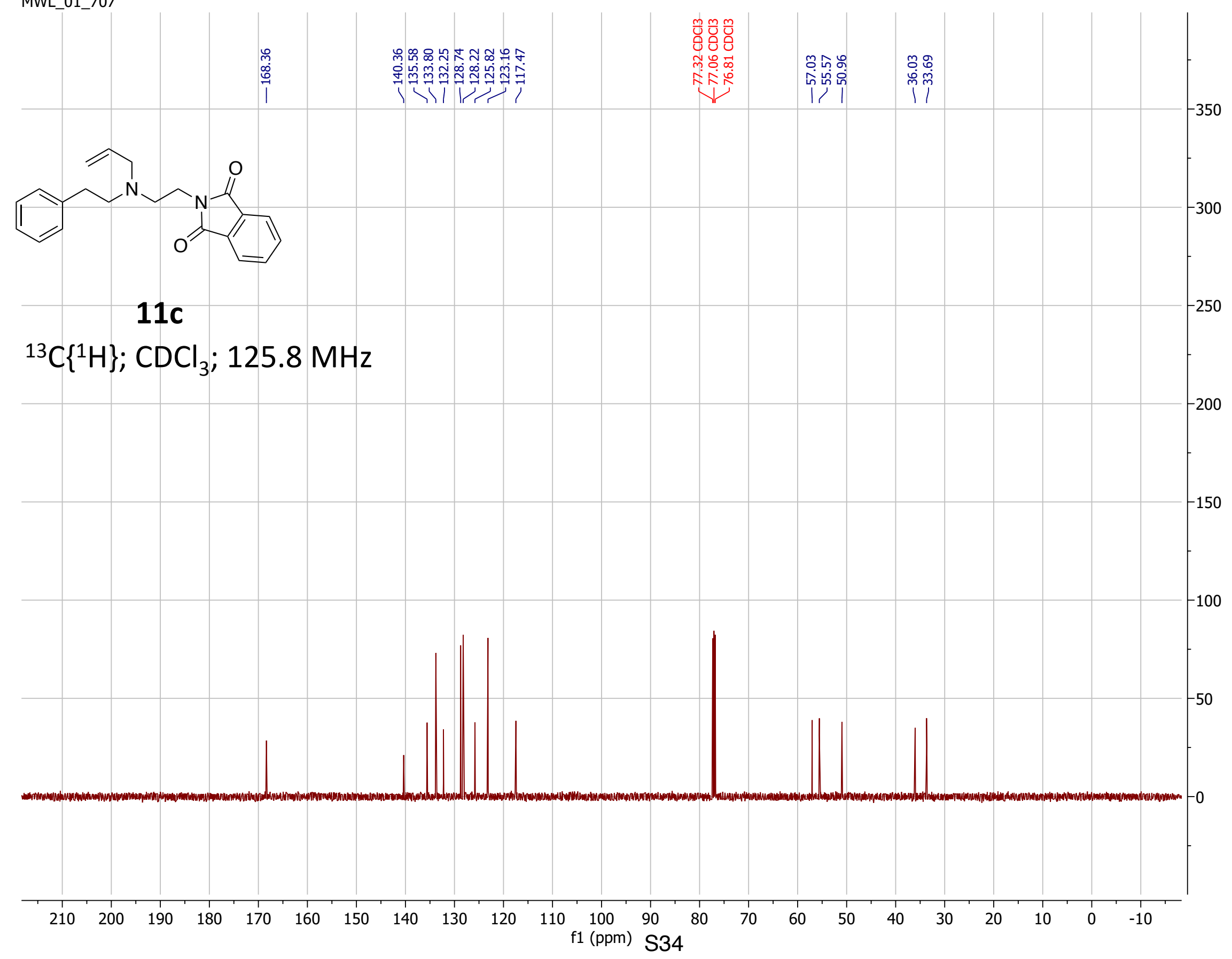




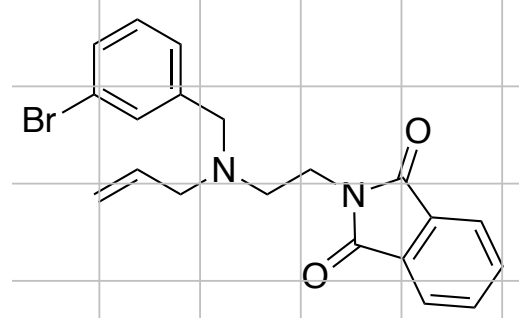

\section{1d}

\section{${ }^{1} \mathrm{H} ; \mathrm{CDCl}_{3} ; 500 \mathrm{MHz}$}

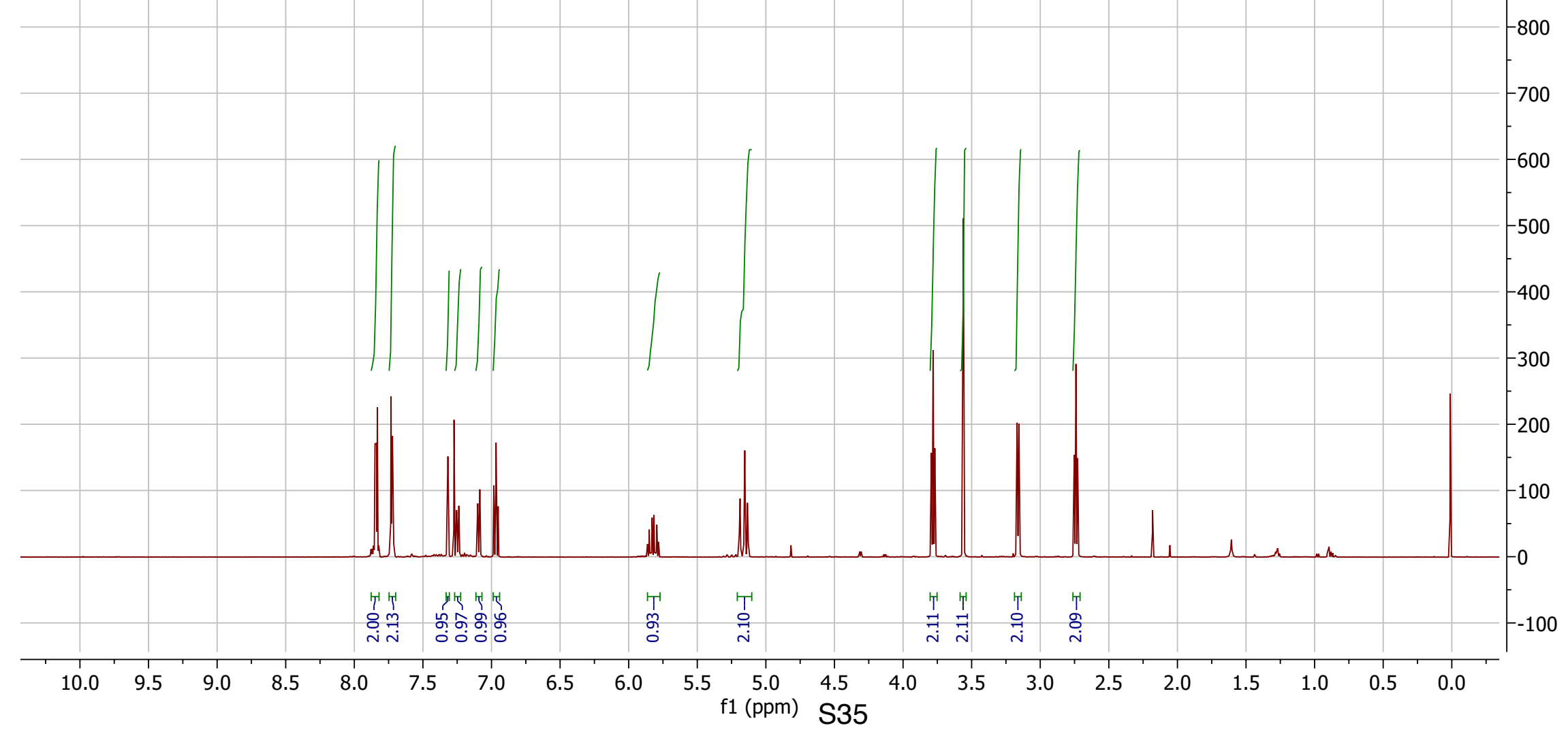




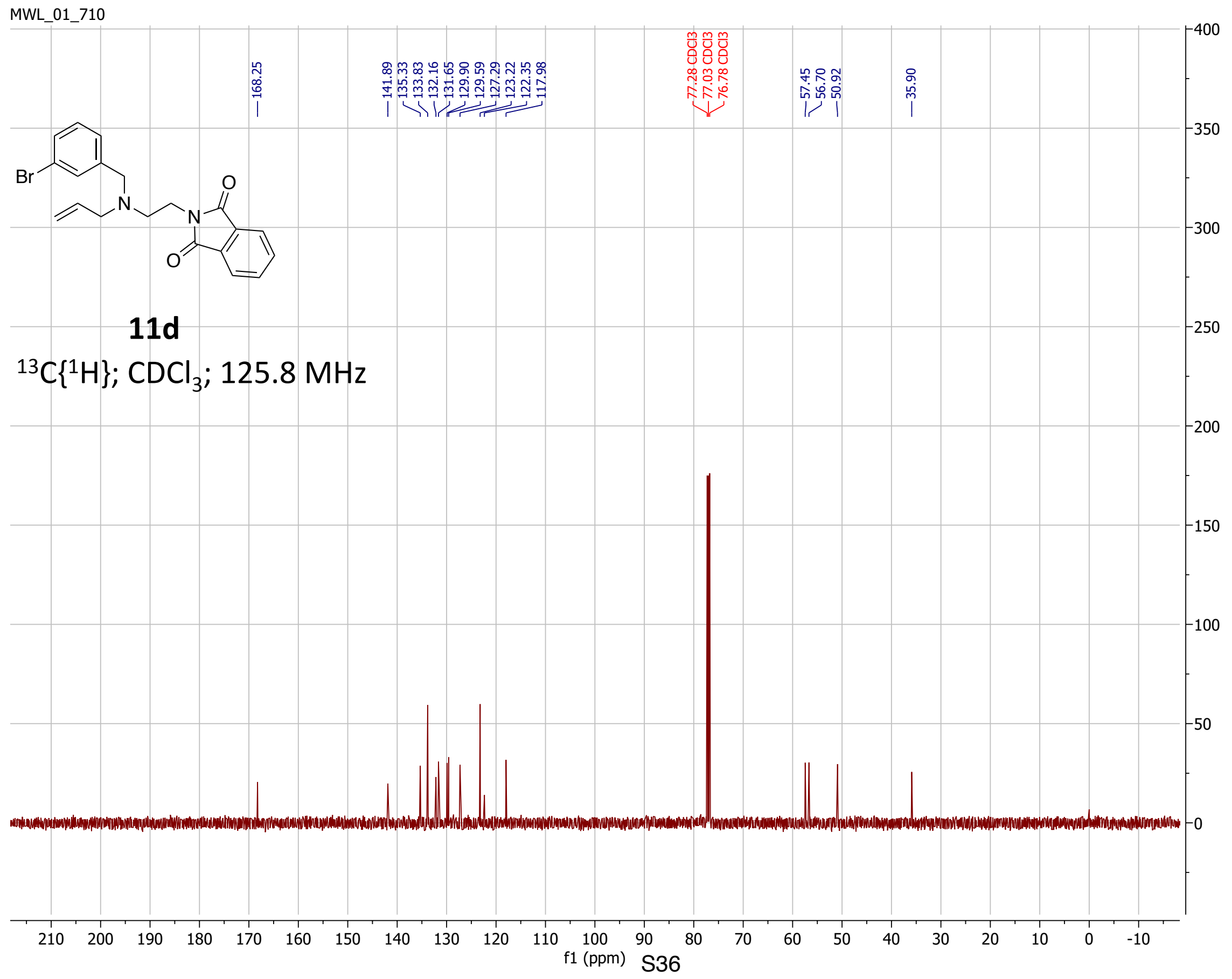


๘
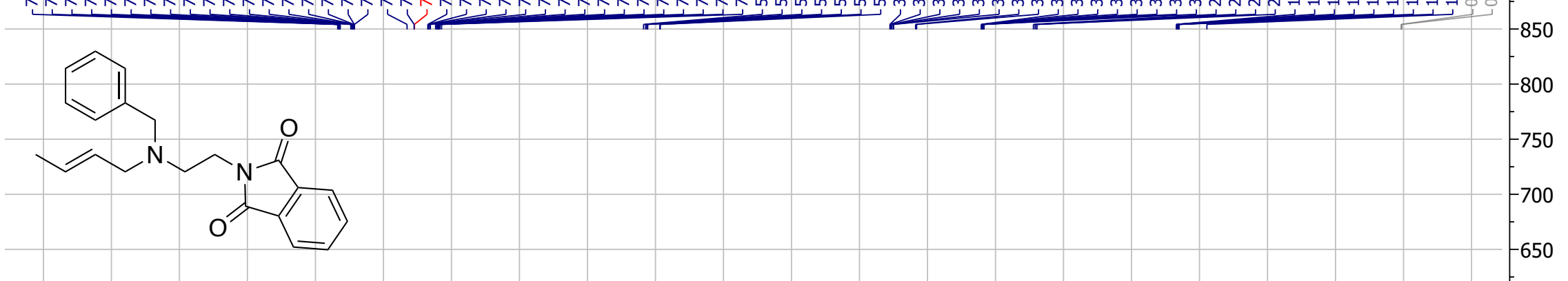

$11 \mathrm{e}$

${ }^{1} \mathrm{H} ; \mathrm{CDCl}_{3} ; 500 \mathrm{MHz}$

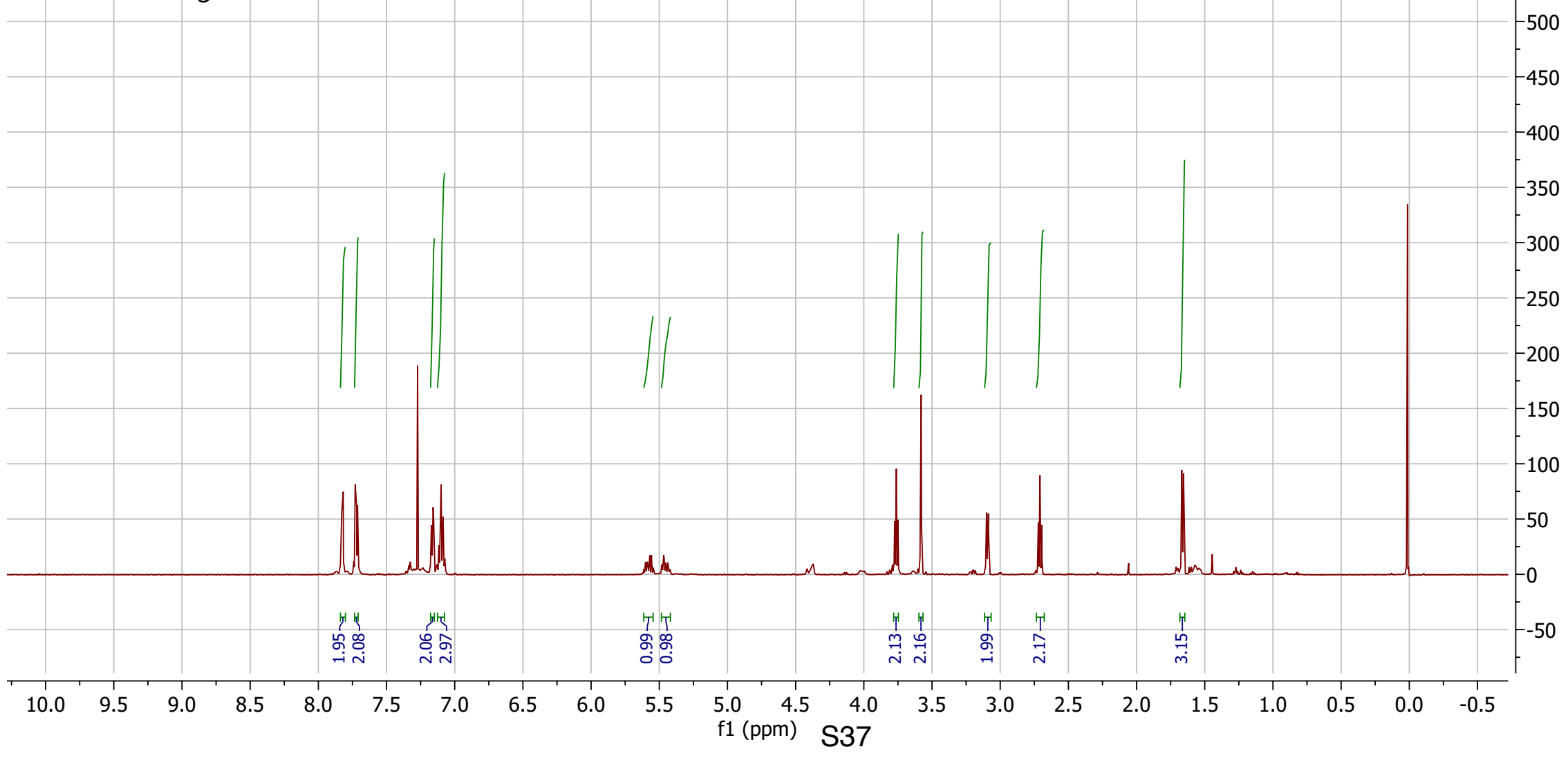




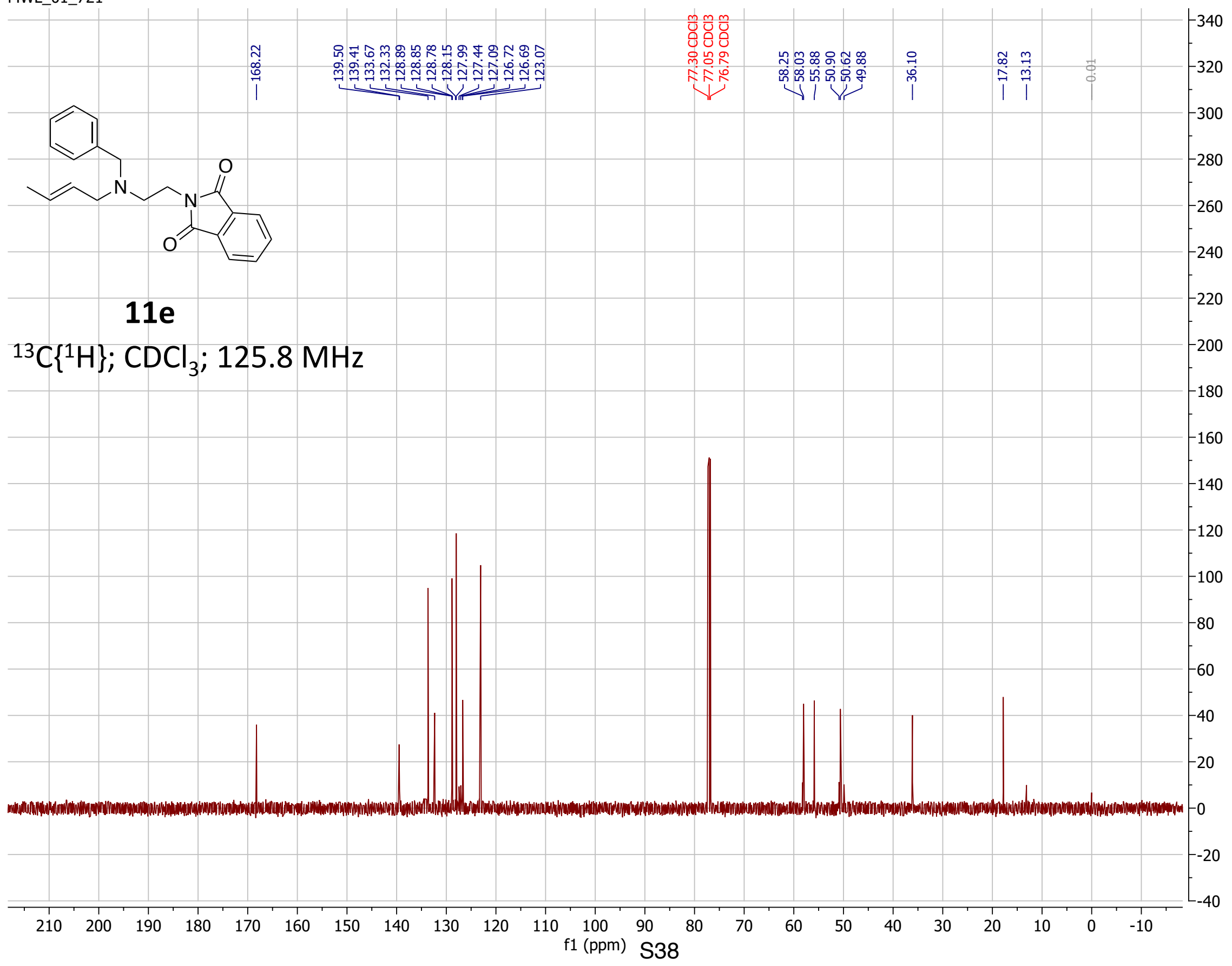




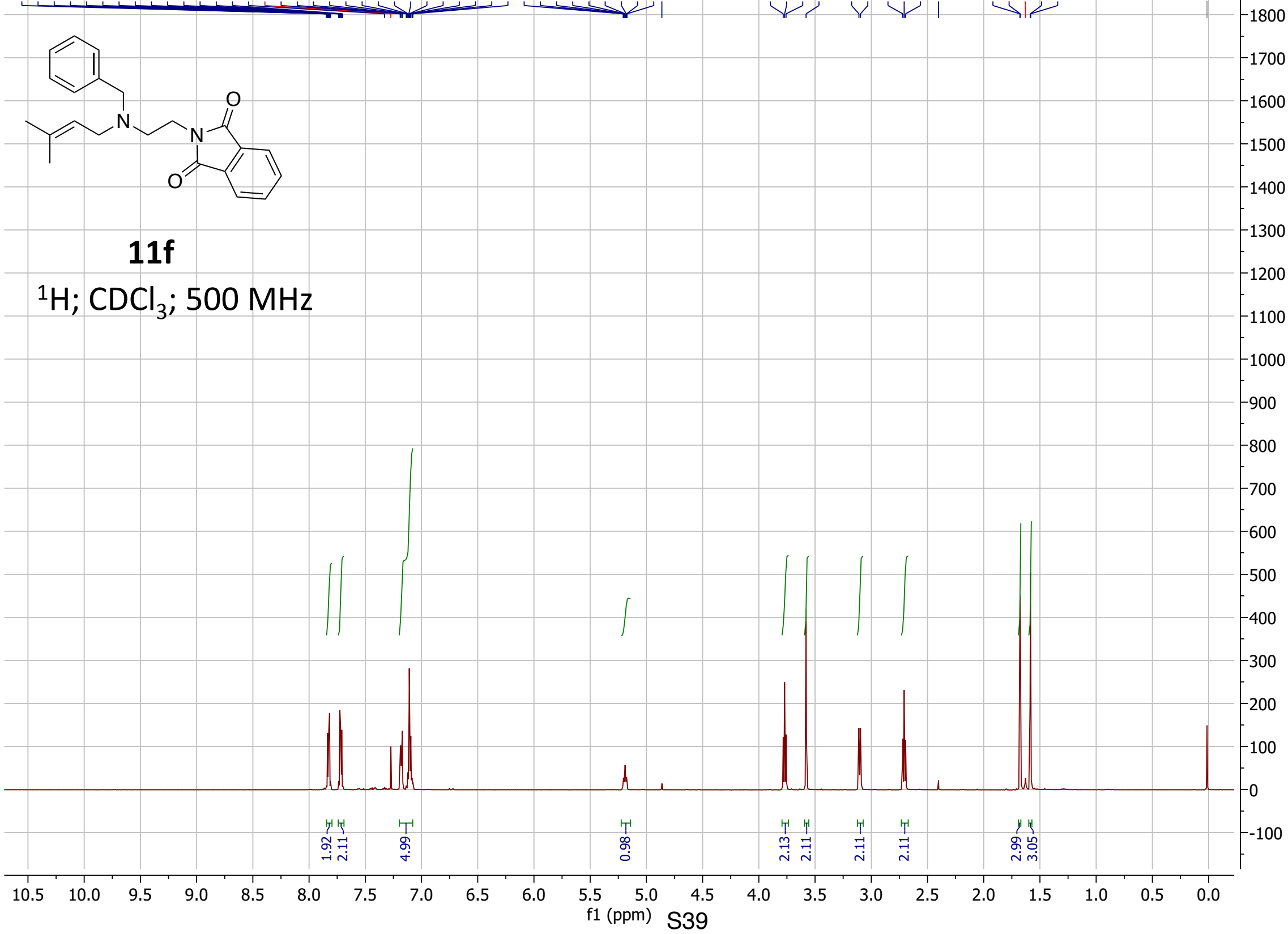




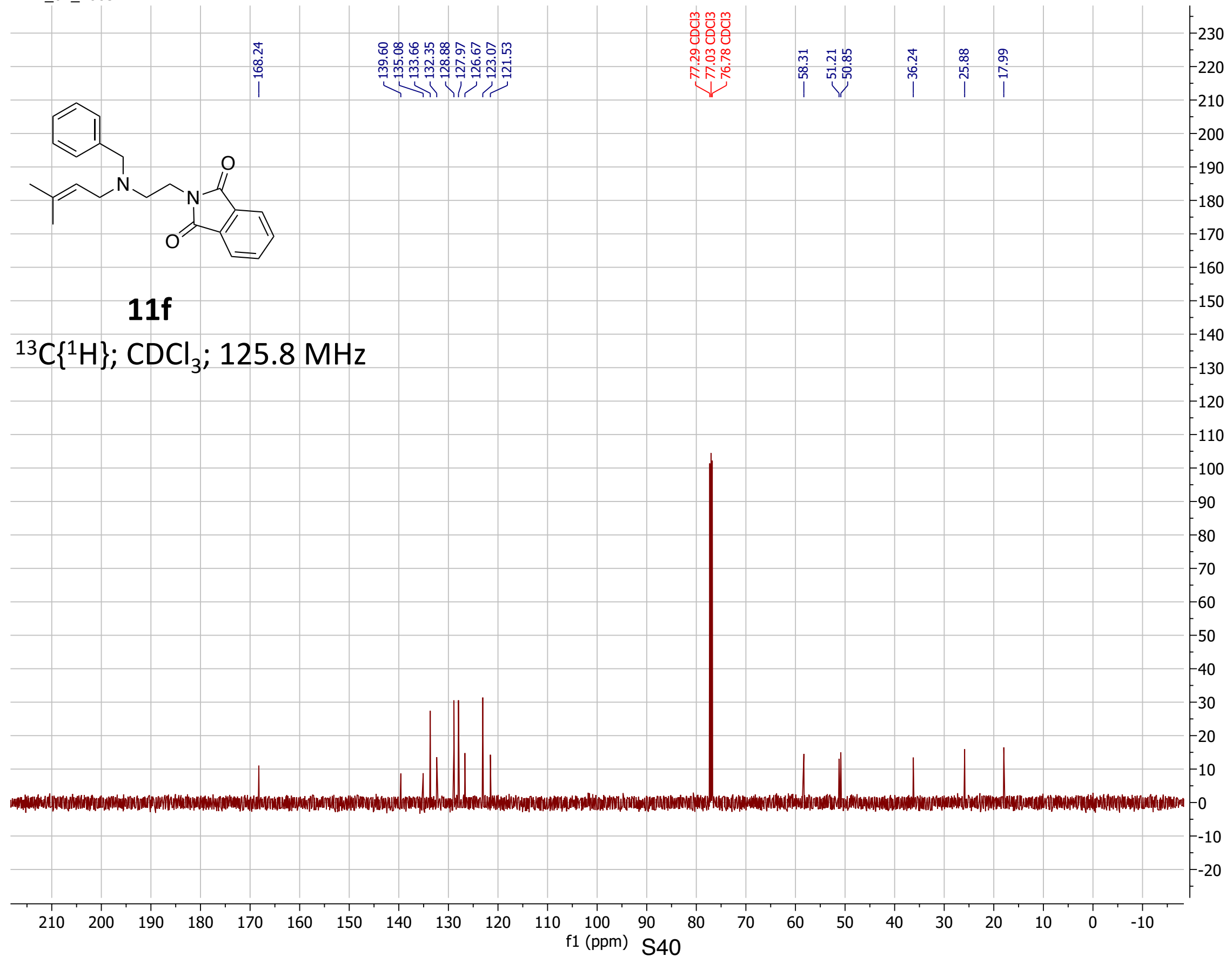




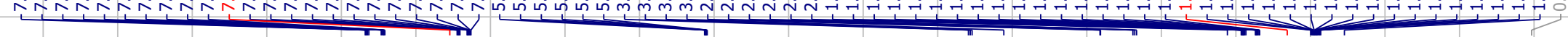

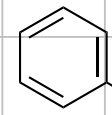<smiles>CCCCN(CCC)CCN1C(=O)c2ccccc2C1=O</smiles>

\section{$11 \mathrm{~g}$}

${ }^{1} \mathrm{H} ; \mathrm{CDCl}_{3} ; 500 \mathrm{MHz}$

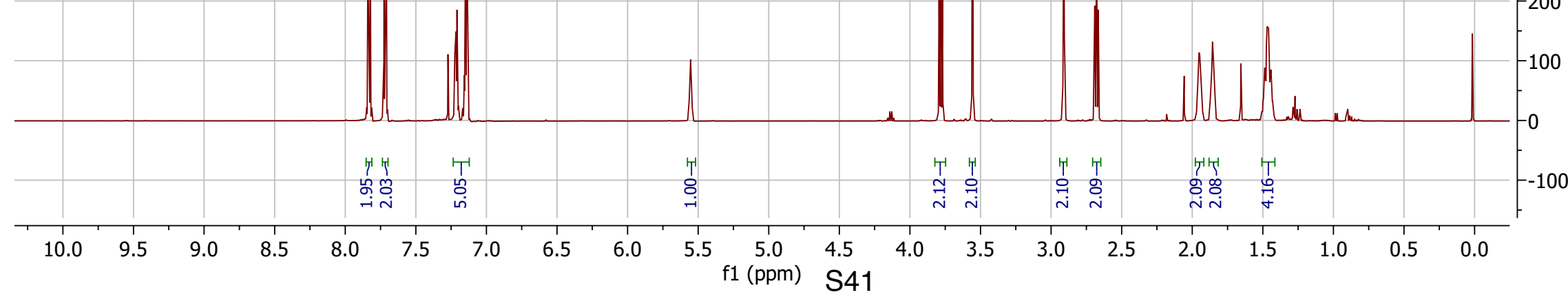




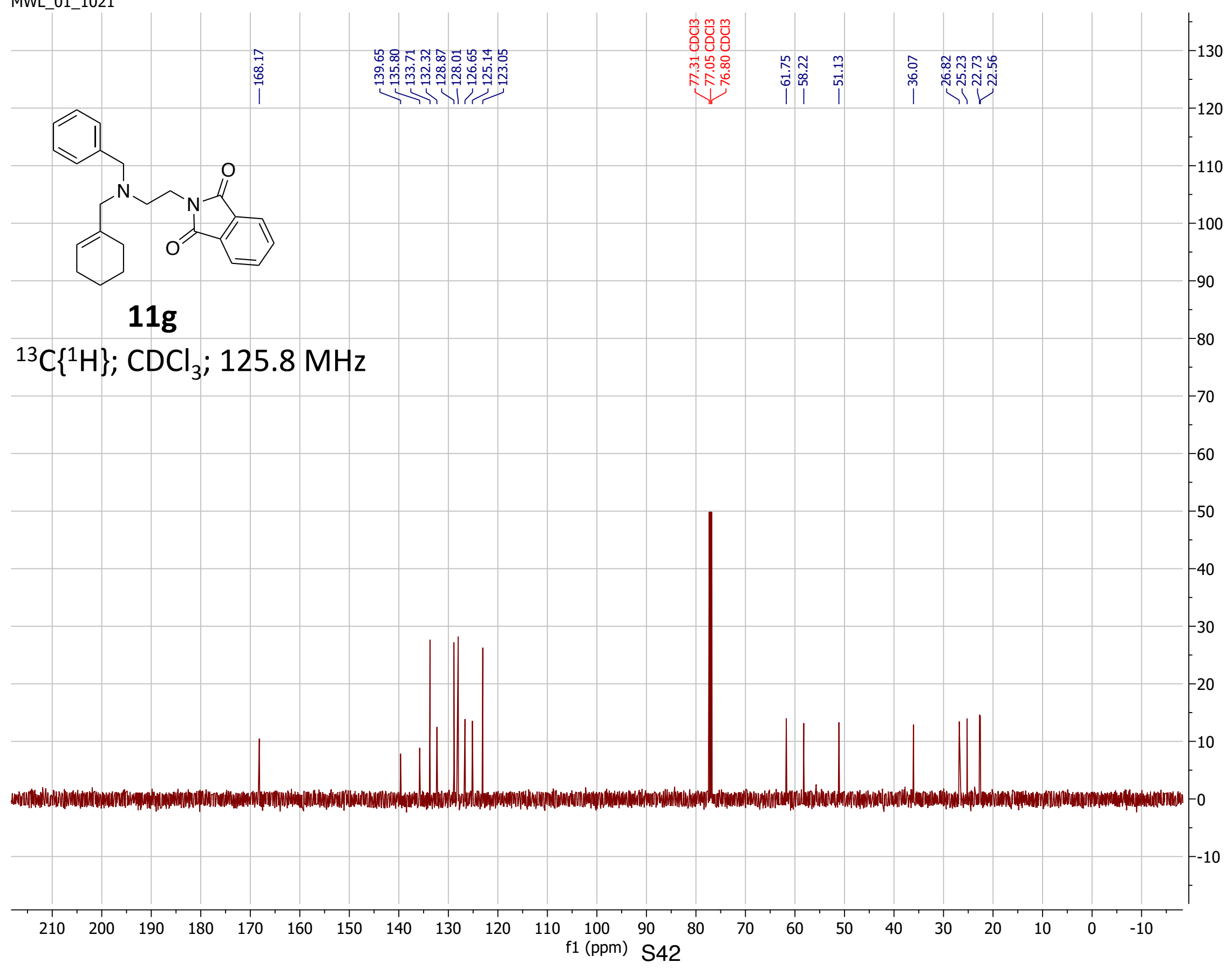




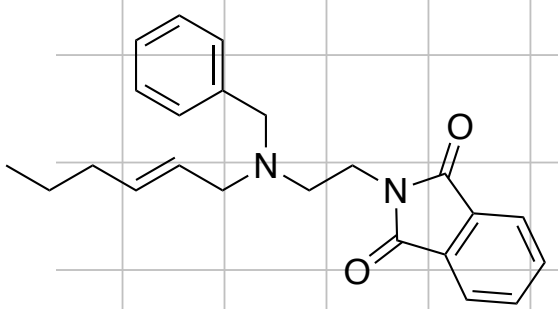

$11 \mathrm{~h}$

\section{${ }^{1} \mathrm{H} ; \mathrm{CDCl}_{3} ; 500 \mathrm{MHz}$}

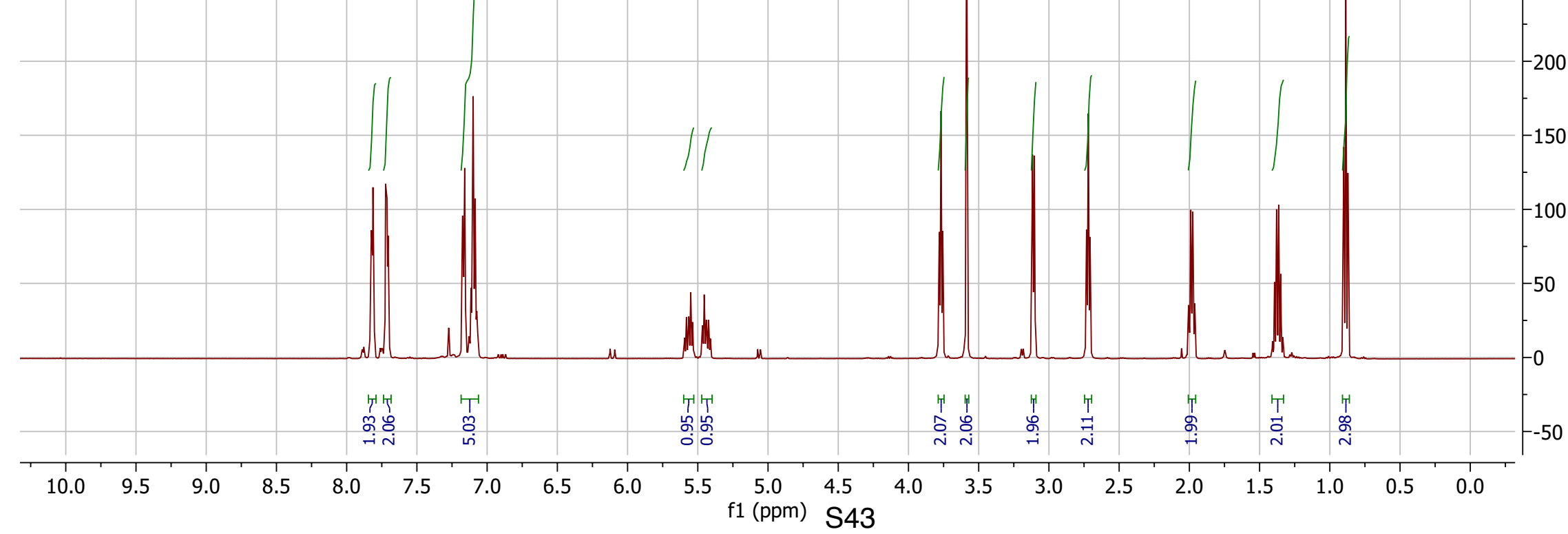




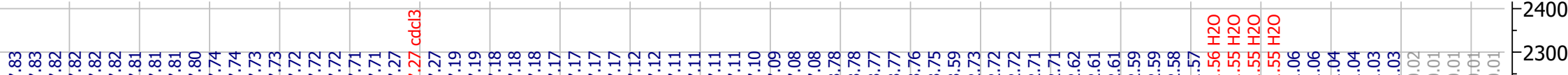
$\underbrace{\infty}$

- -2100

102000

$\sim \sim_{N} \mu$ 011

\section{1i}

${ }^{1} \mathrm{H} ; \mathrm{CDCl}_{3} ; 500 \mathrm{MHz}$

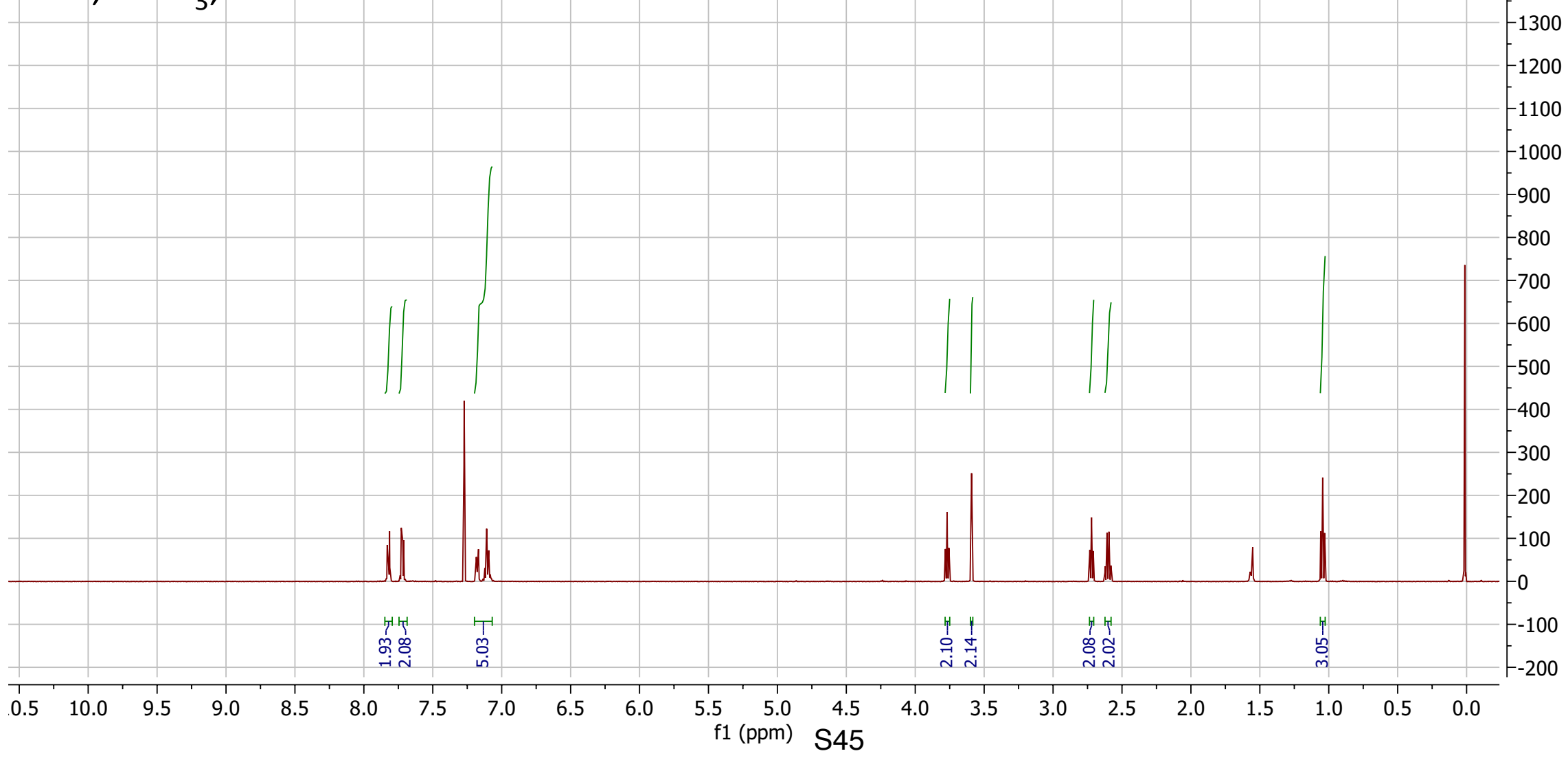



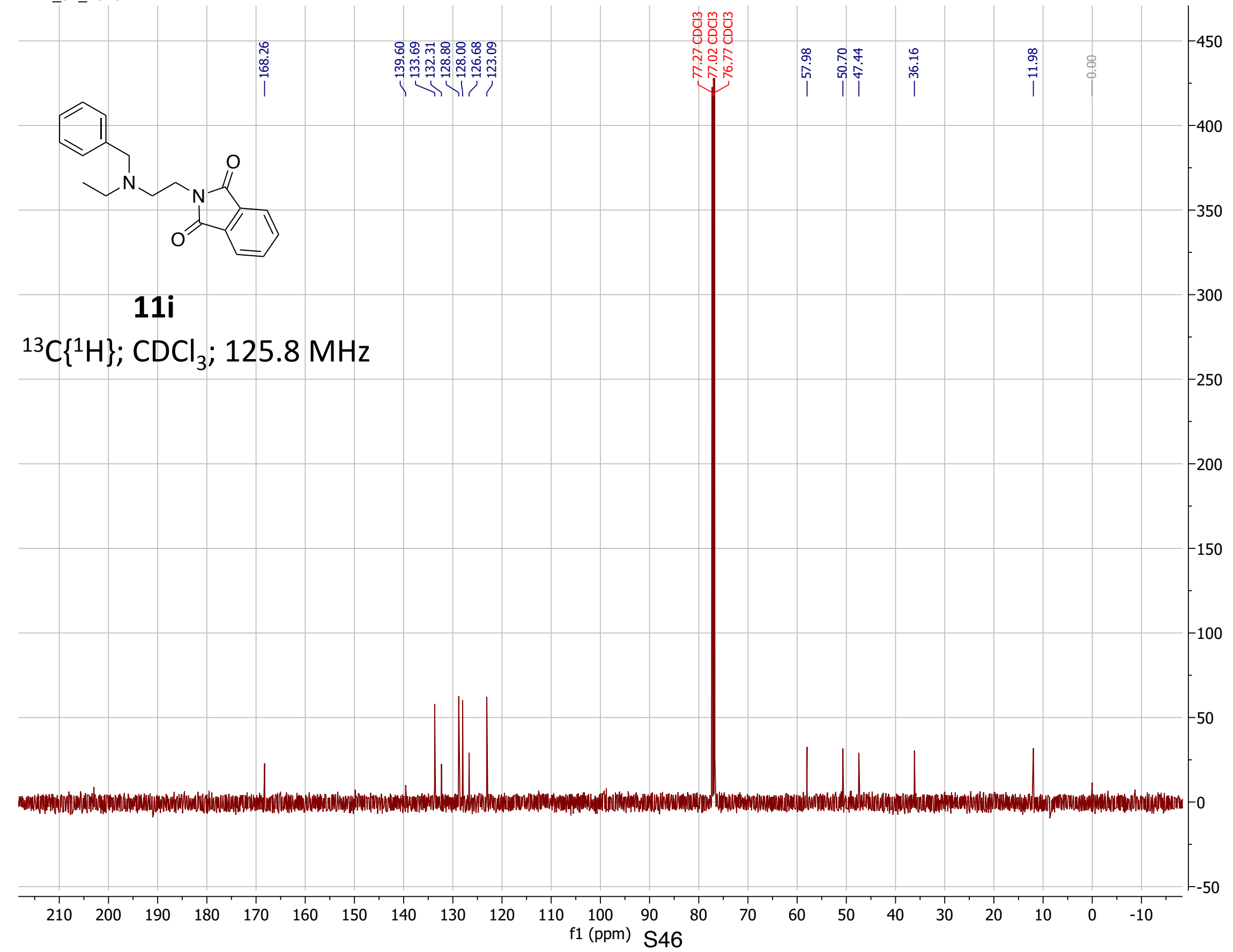


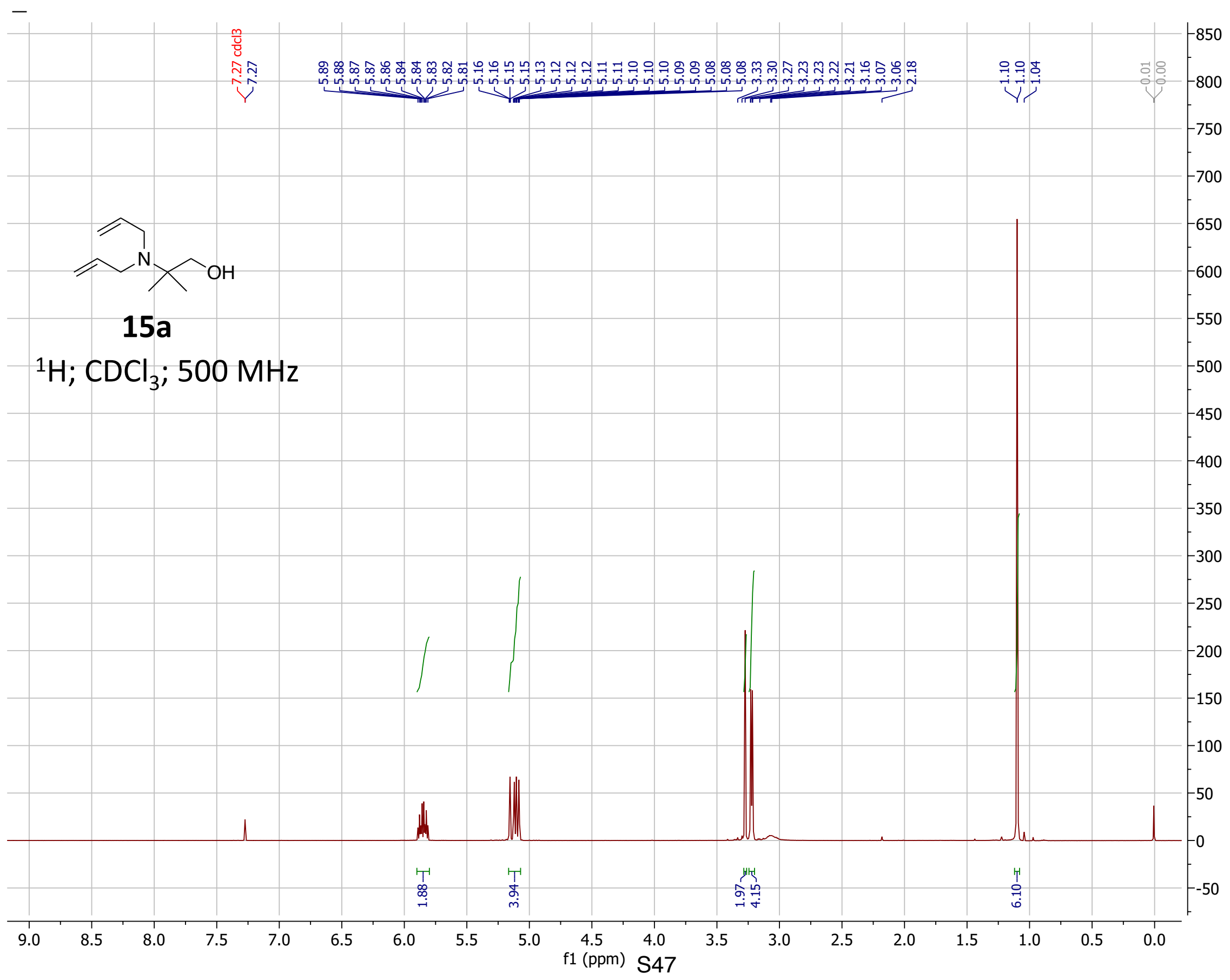




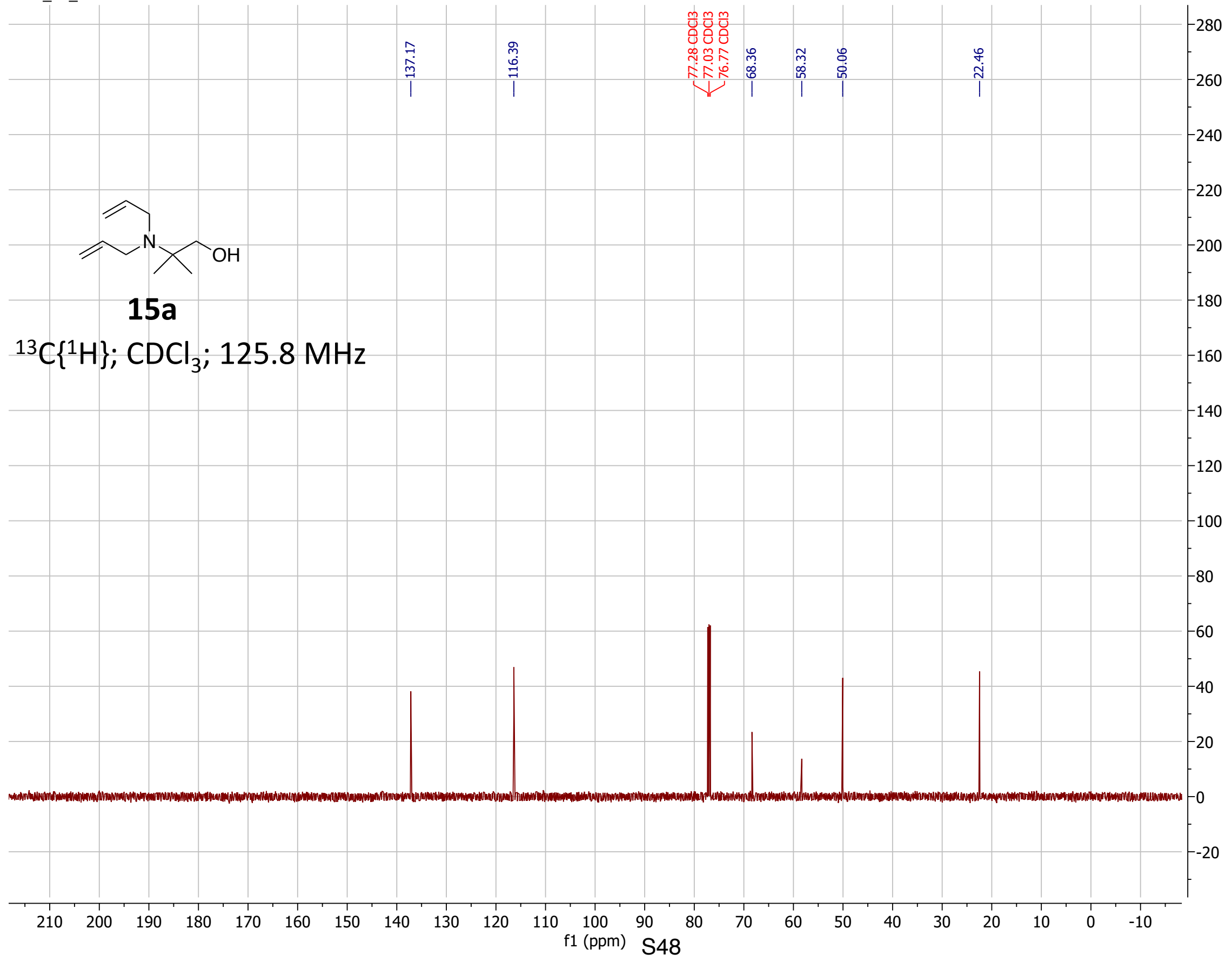




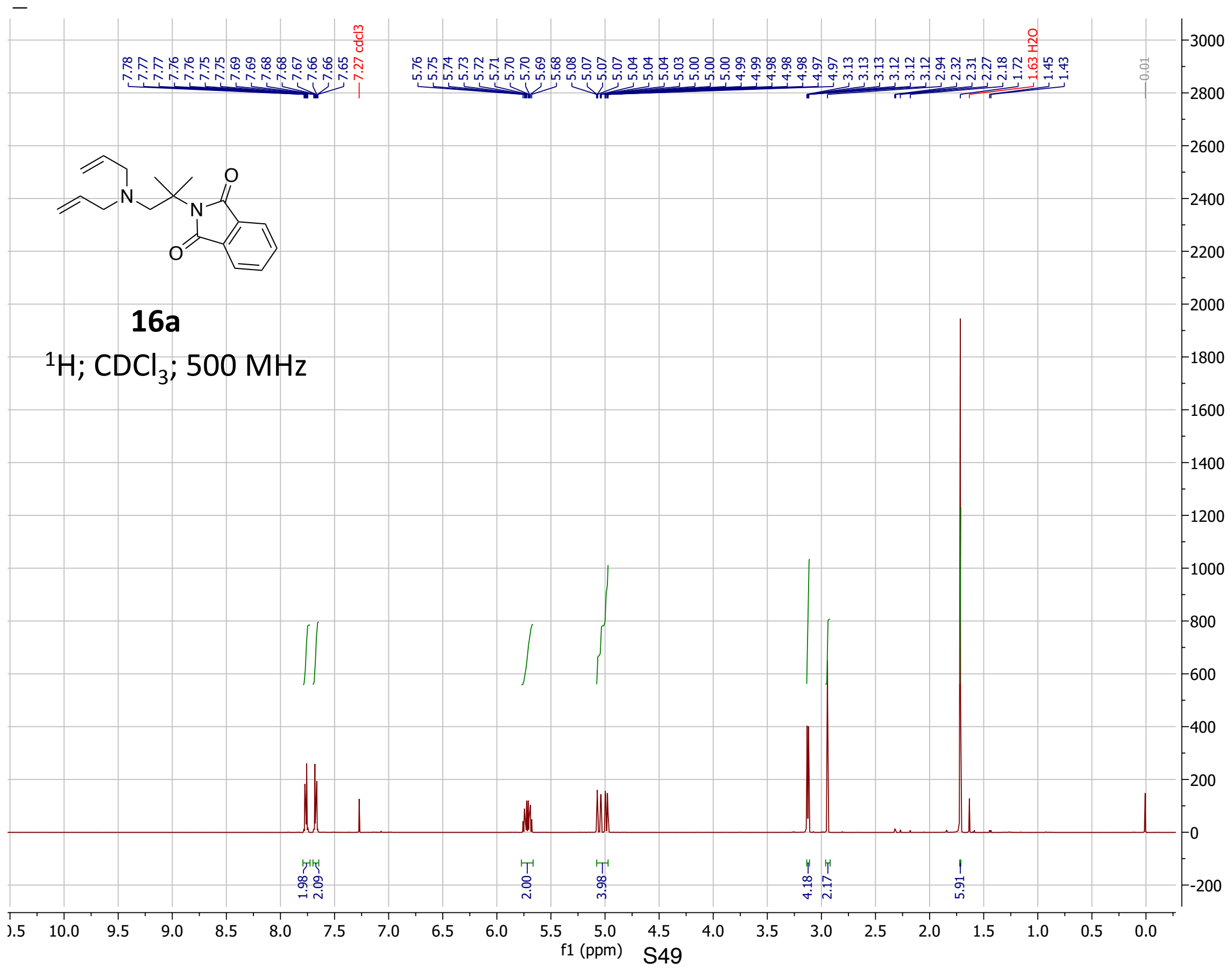




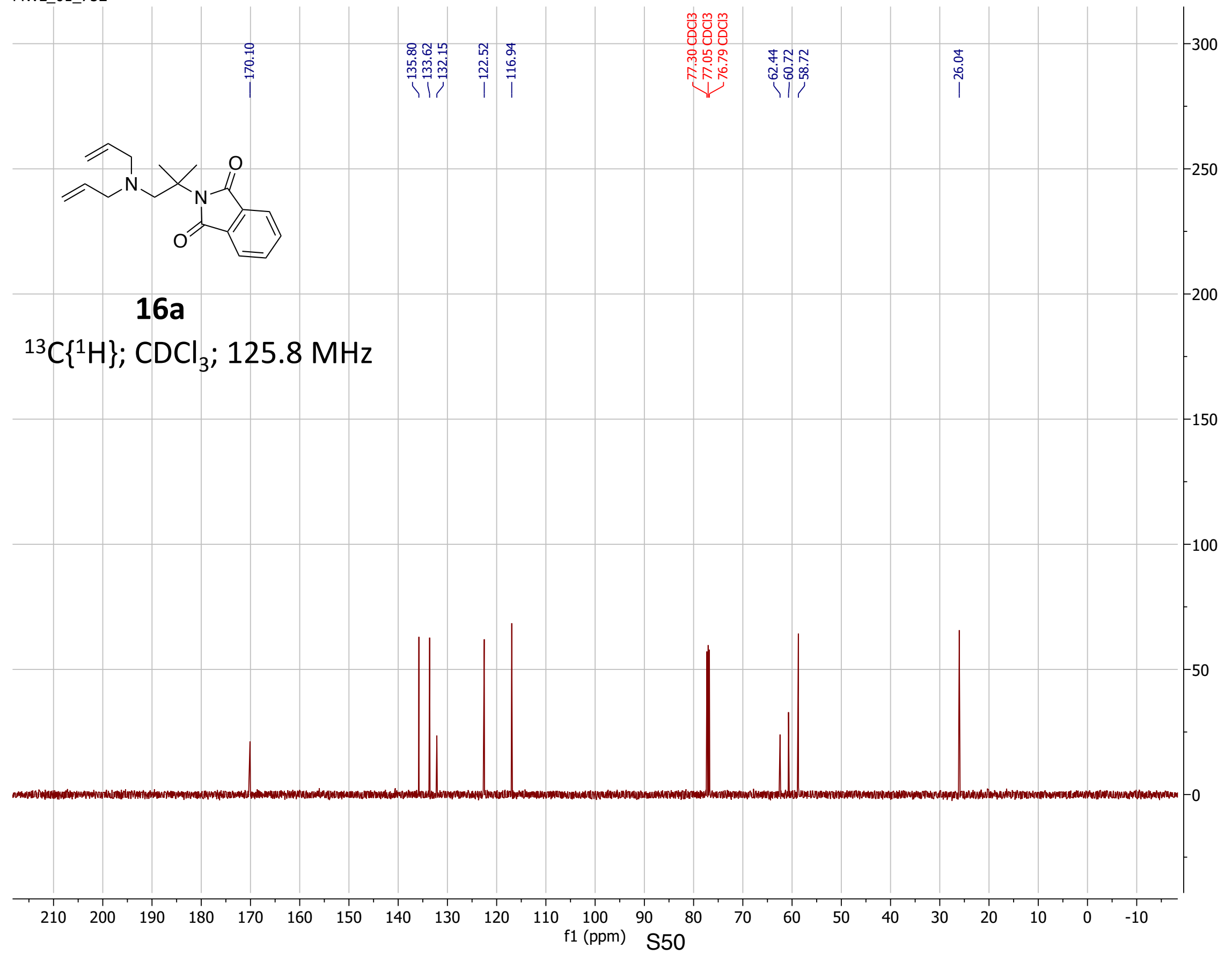




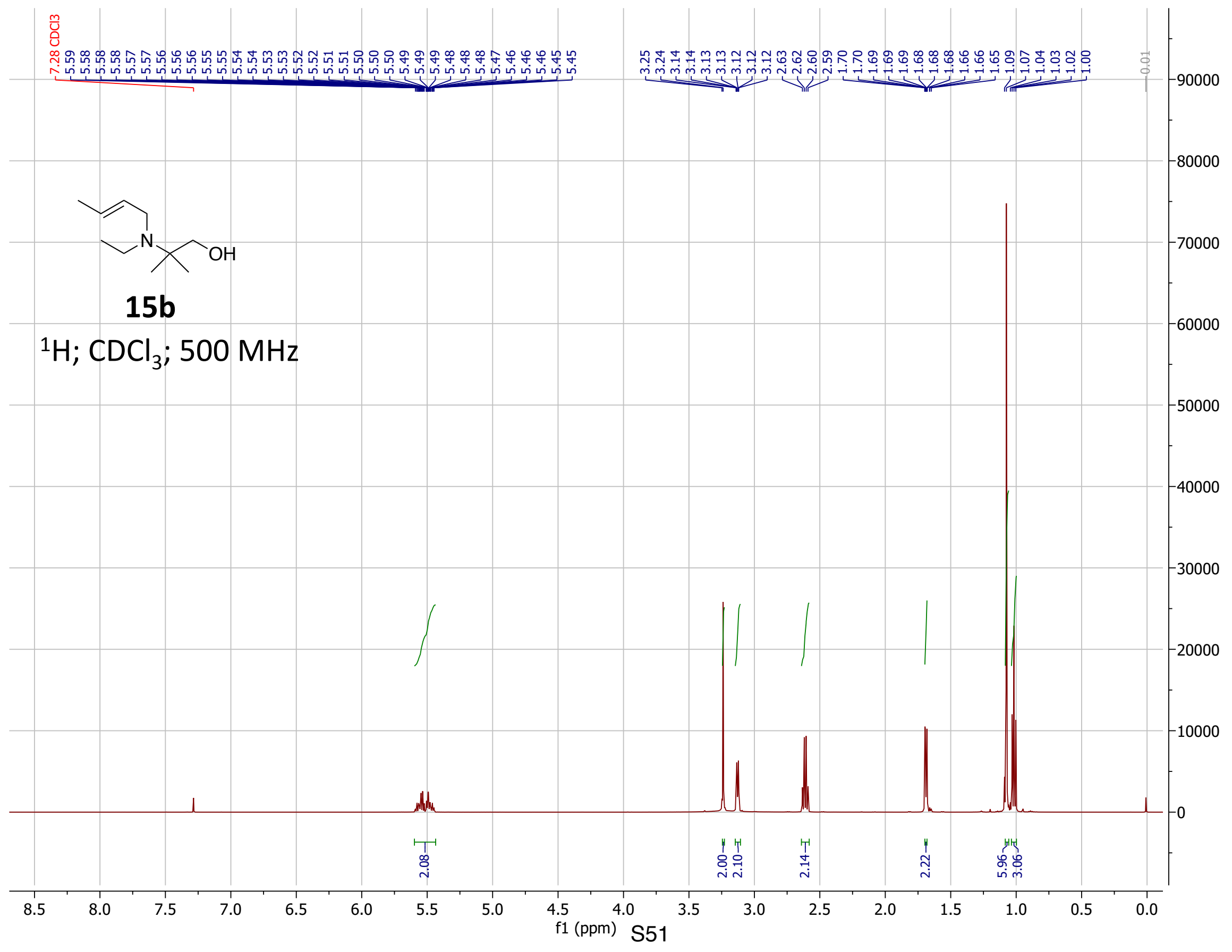




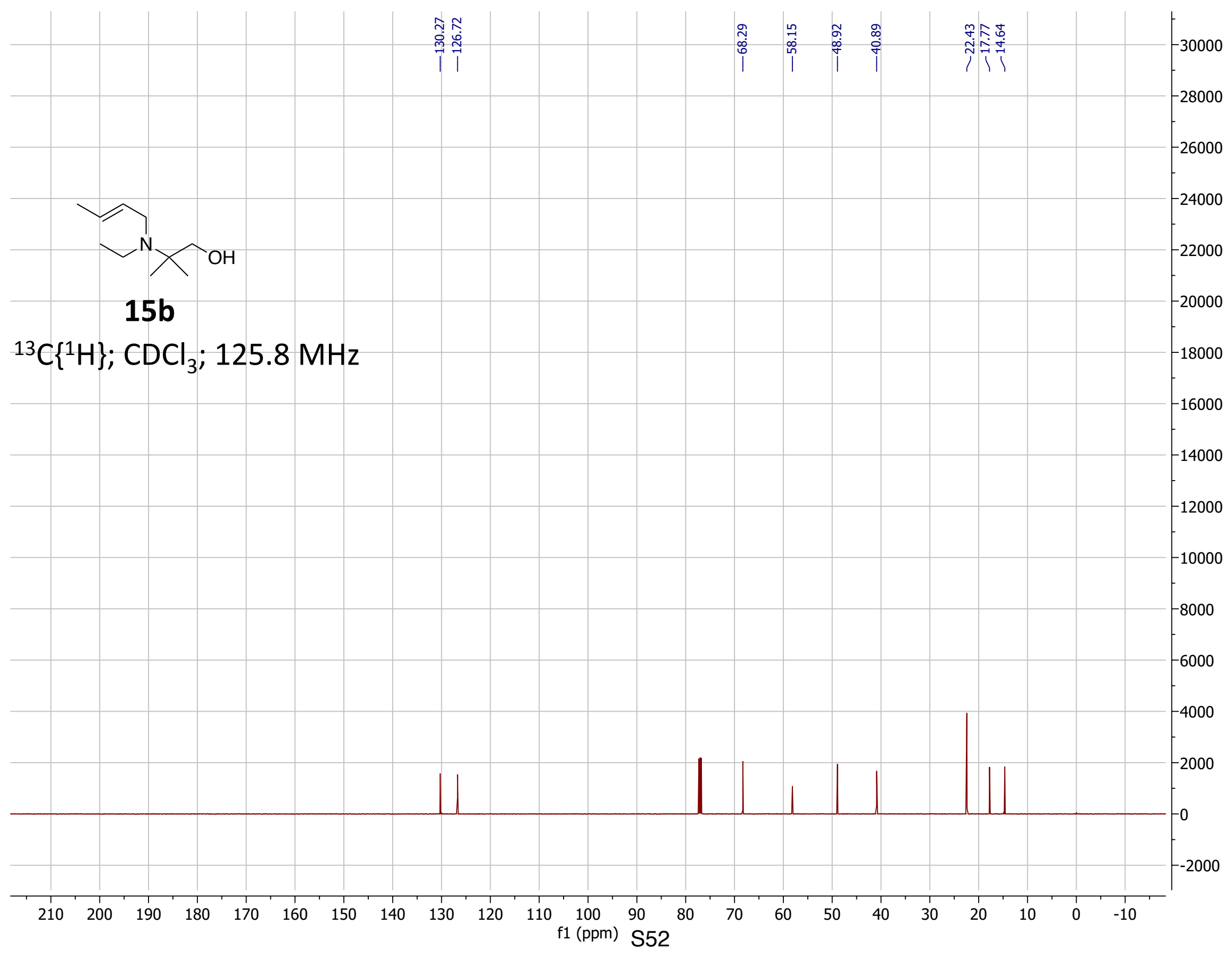




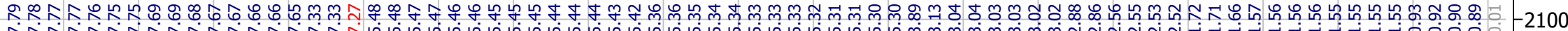

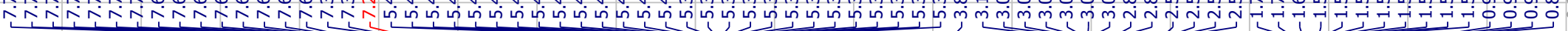

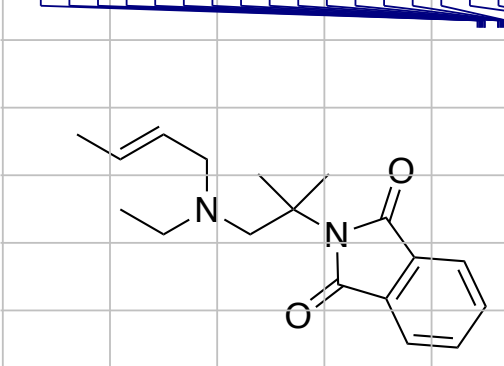

$16 b$

\section{${ }^{1} \mathrm{H} ; \mathrm{CDCl}_{3} ; 500 \mathrm{MHz}$}

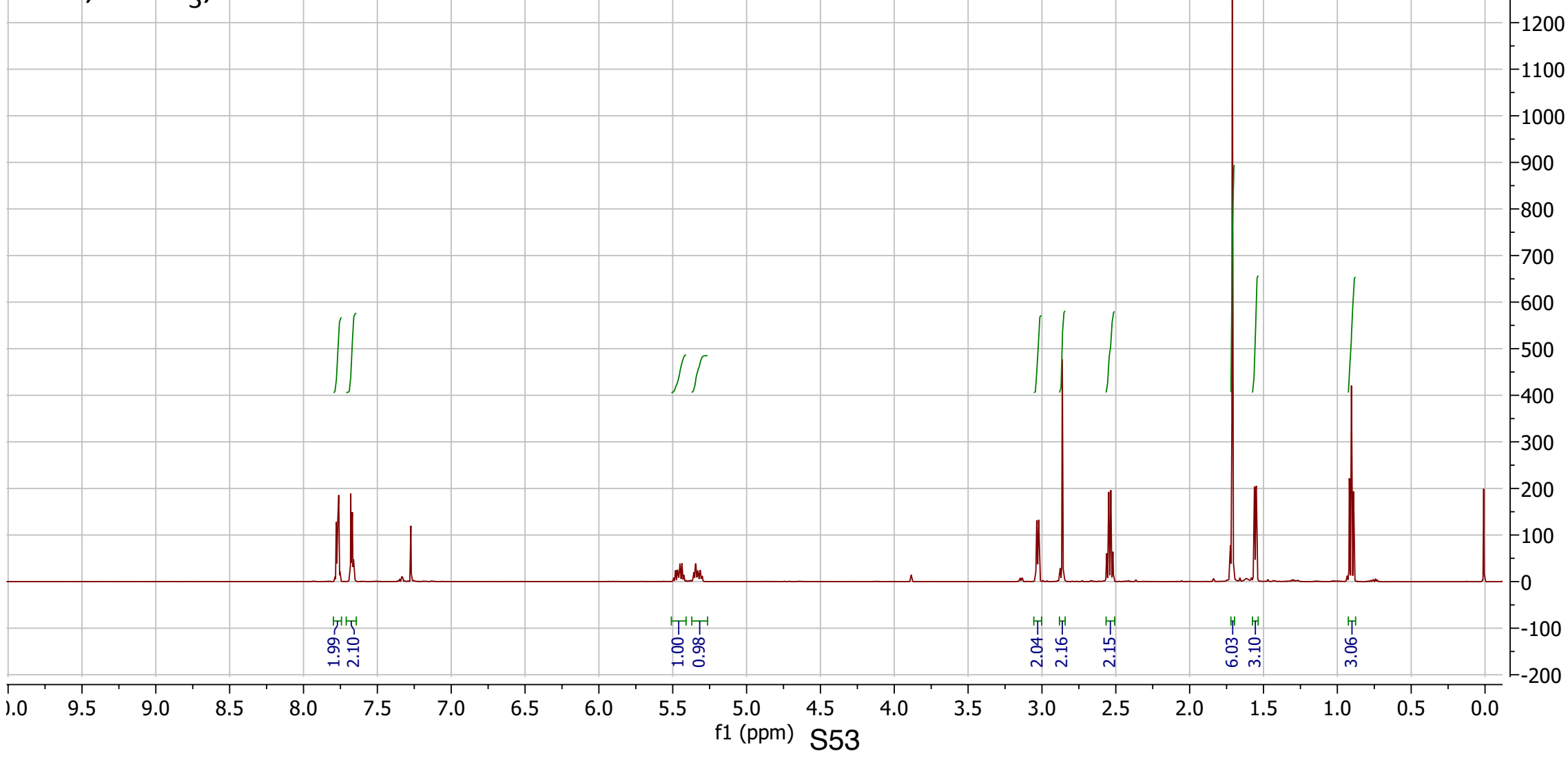



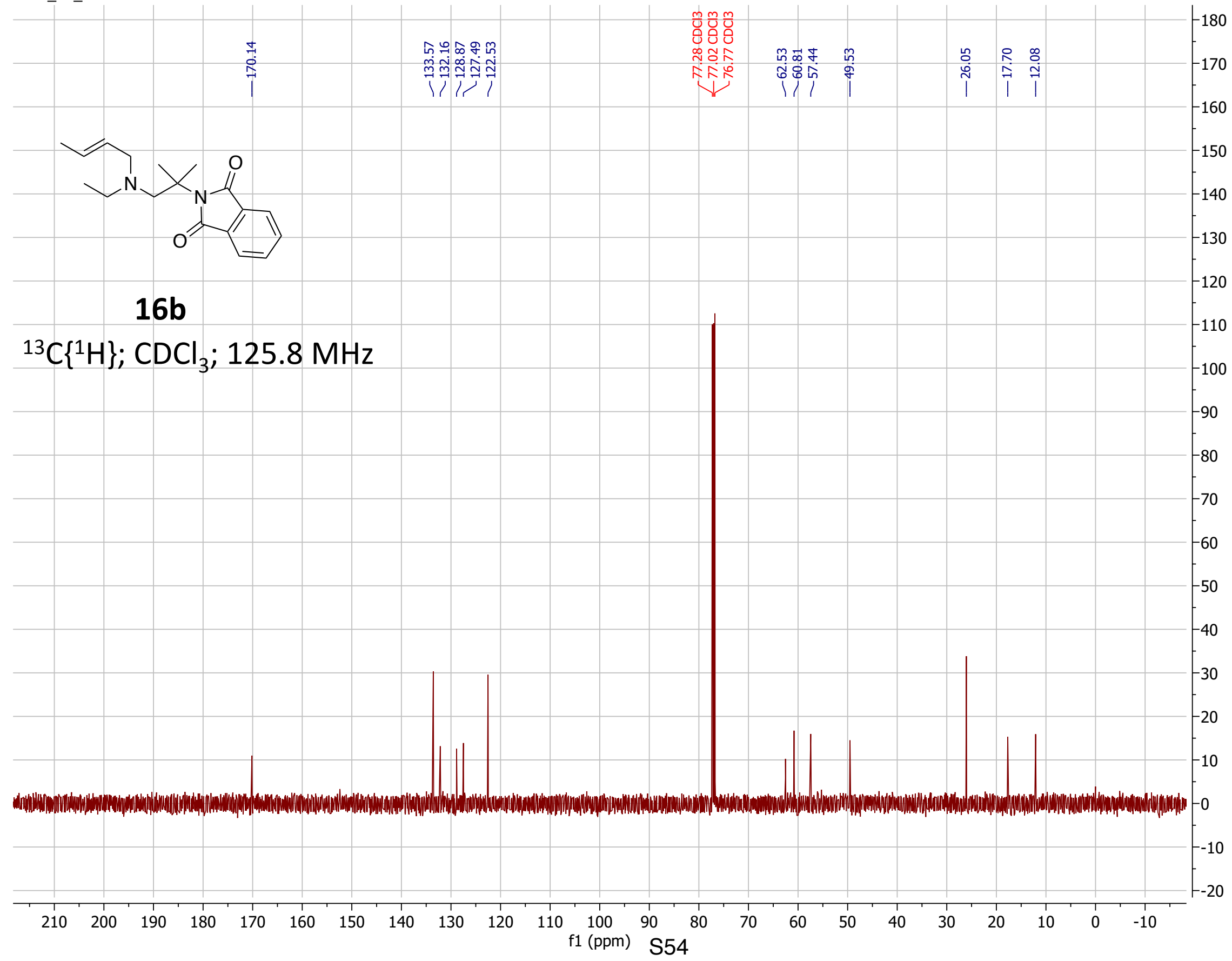


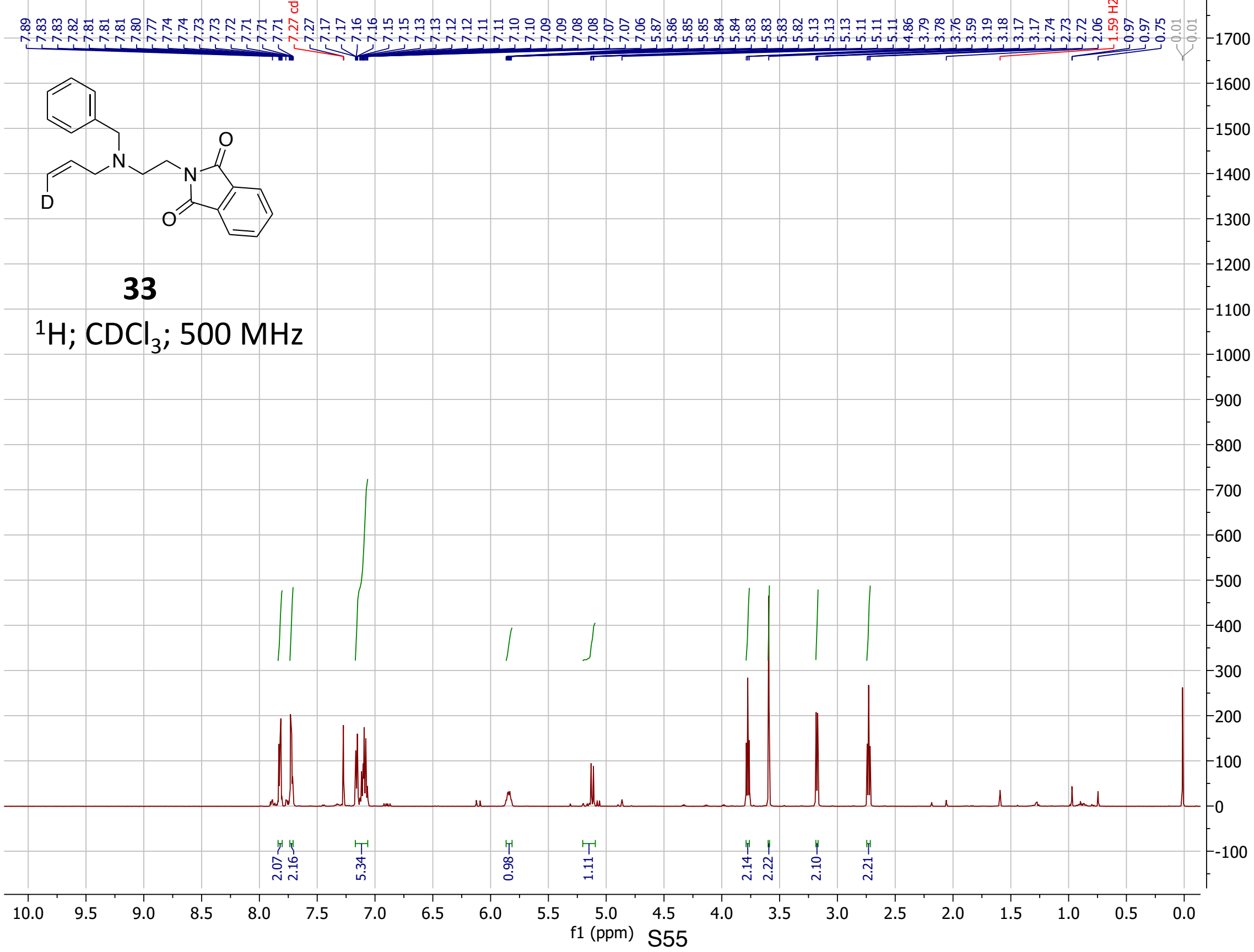




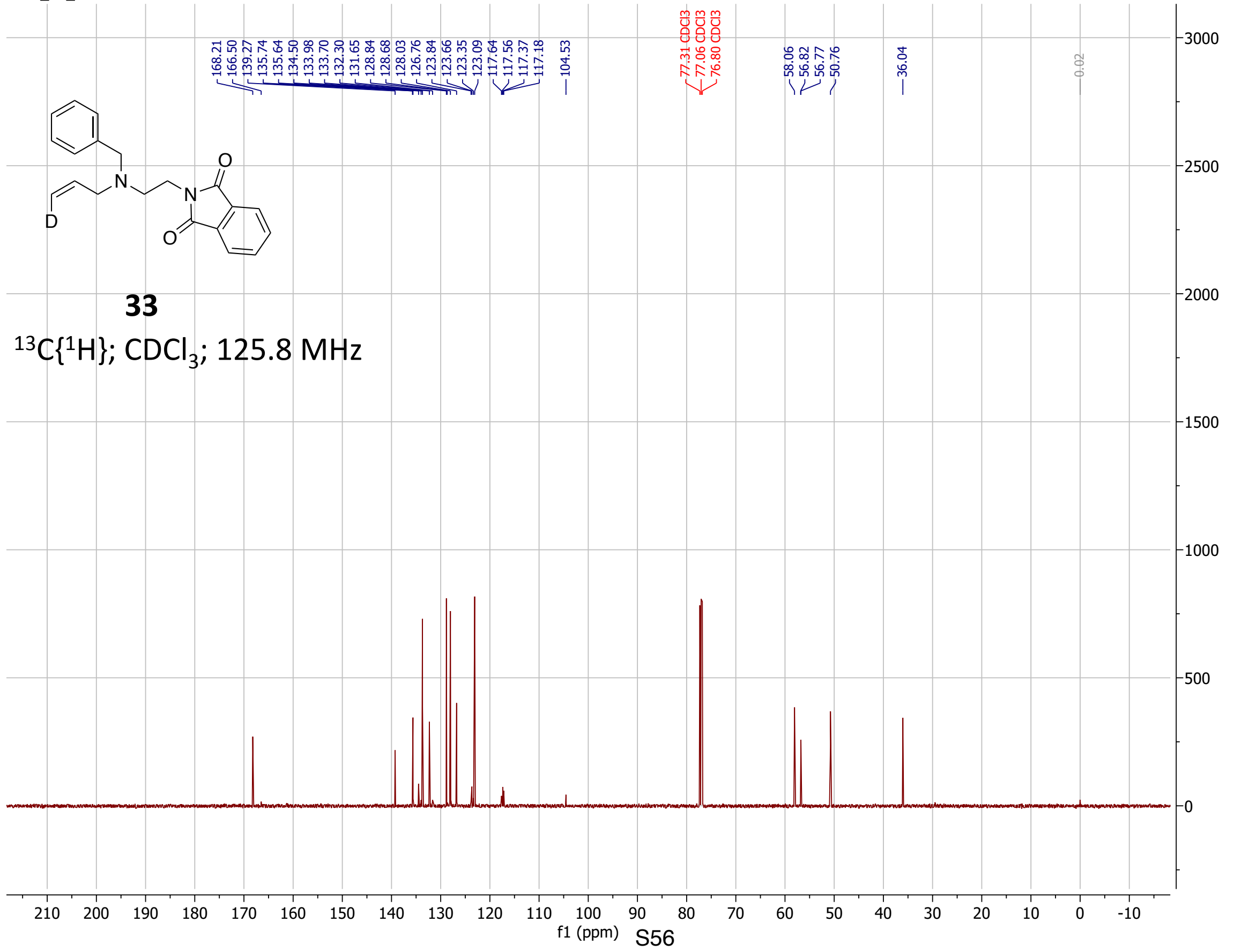




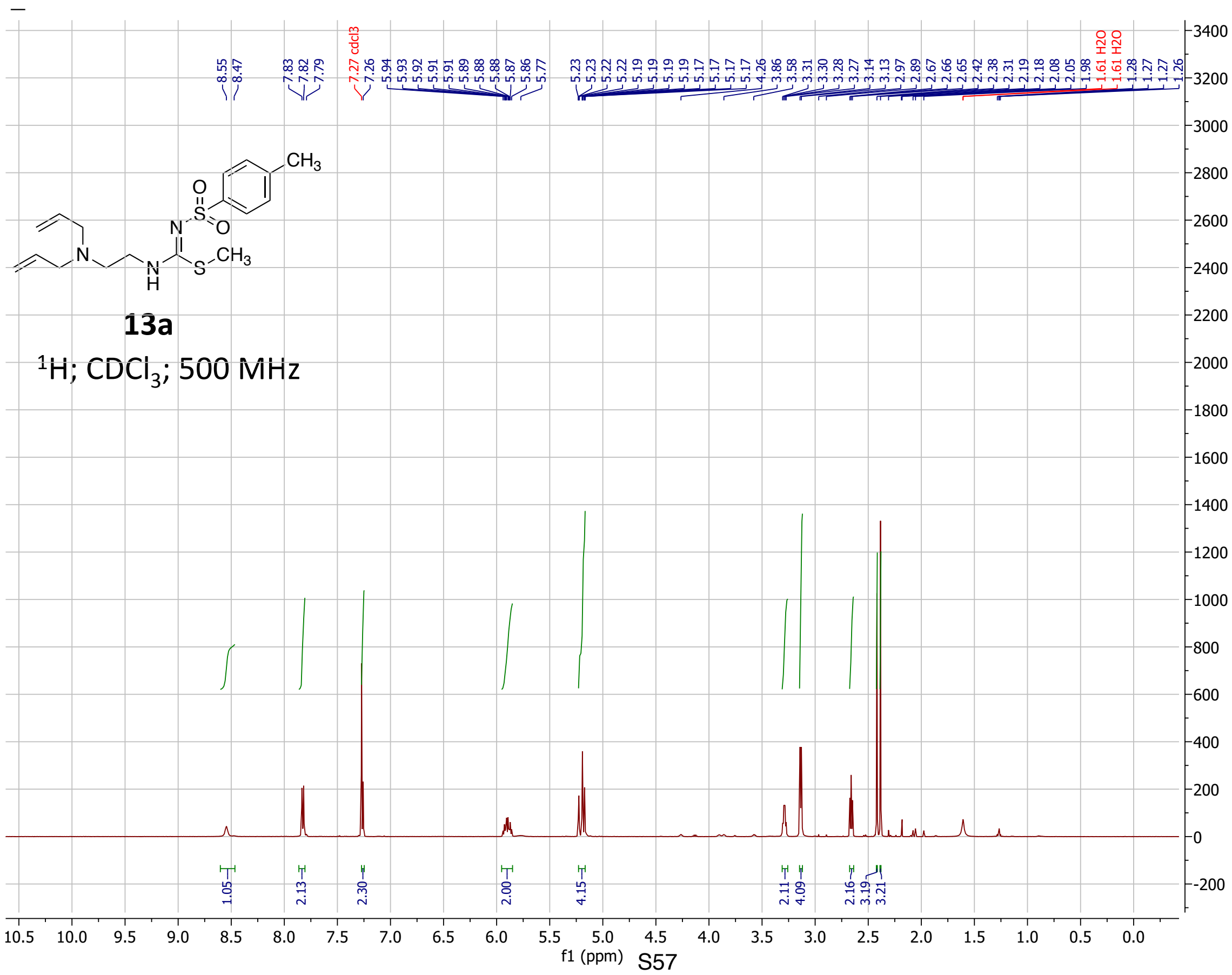




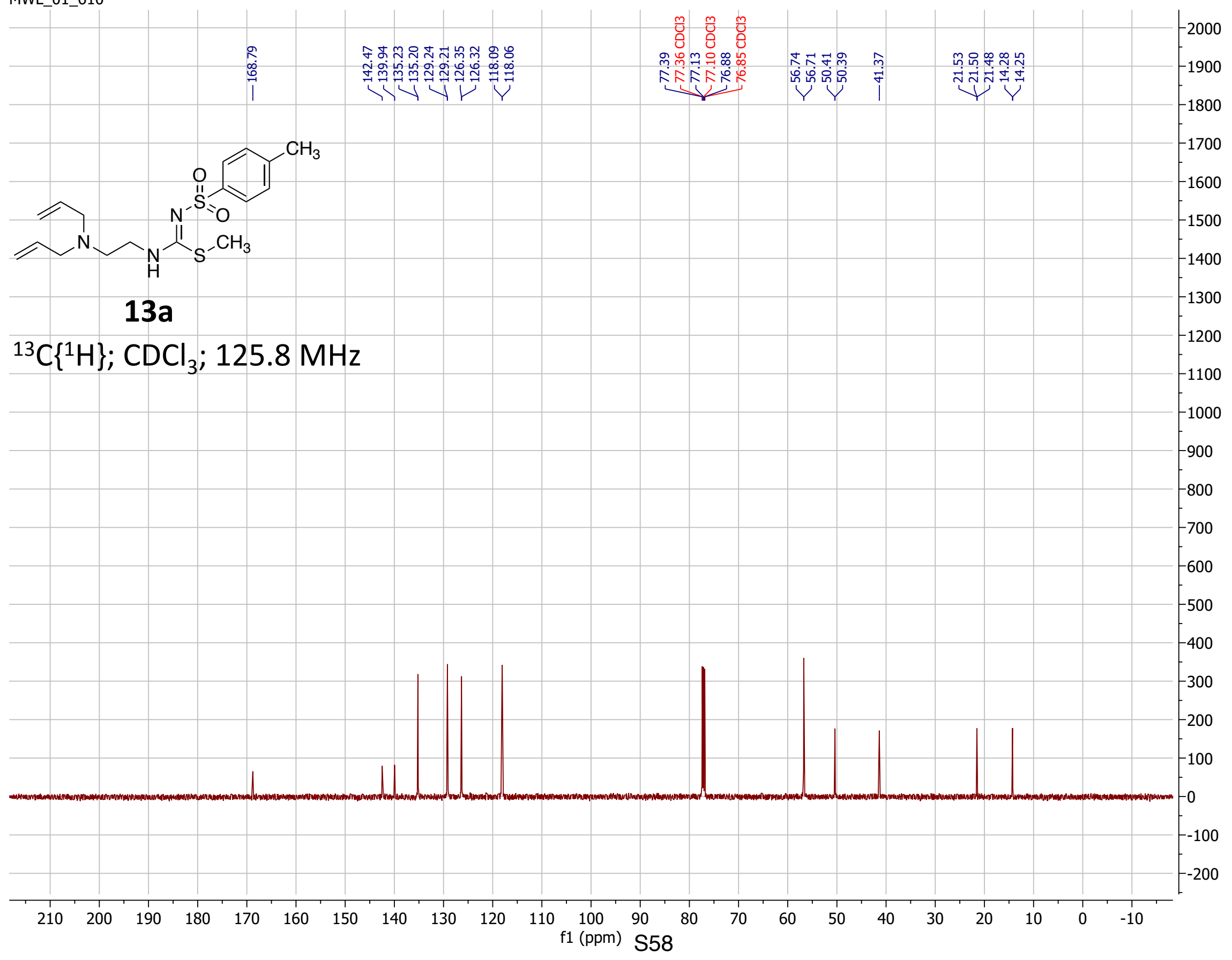




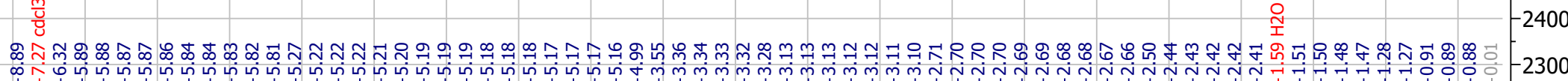

2 (III)

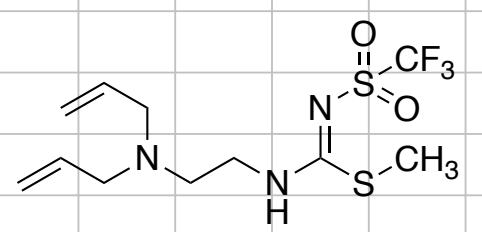

13b

${ }^{1} \mathrm{H} ; \mathrm{CDCl}_{3} ; 500 \mathrm{MHz}$

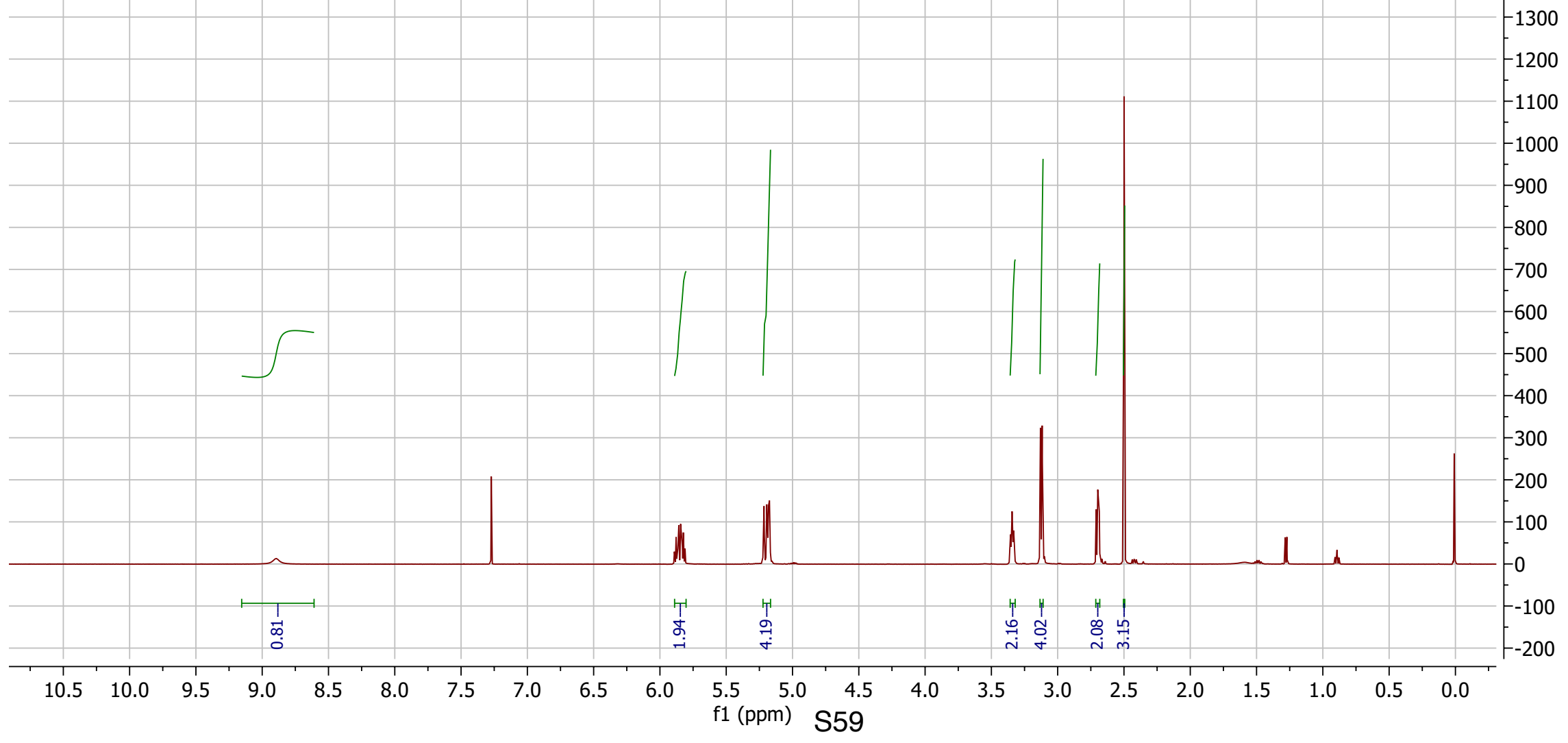




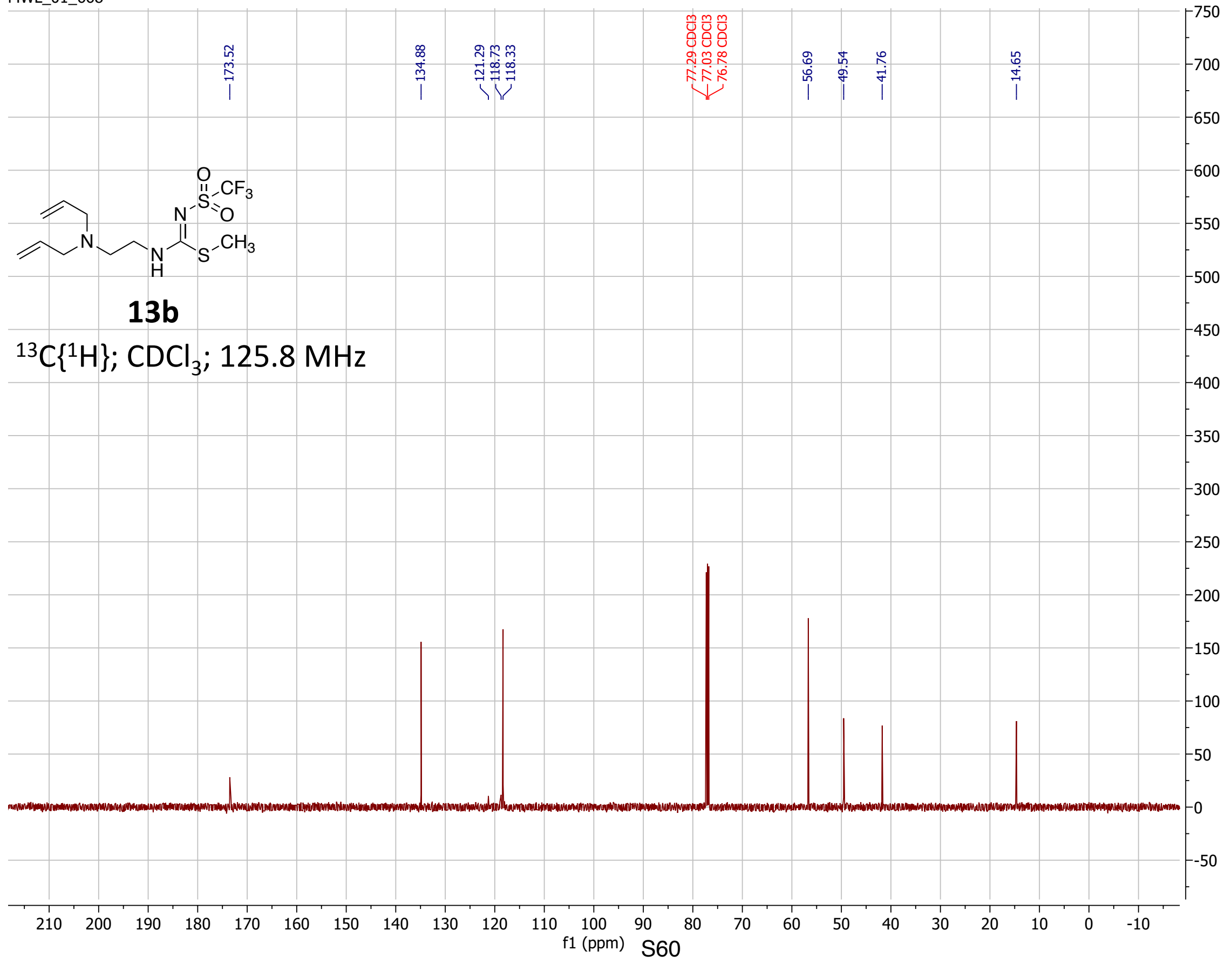




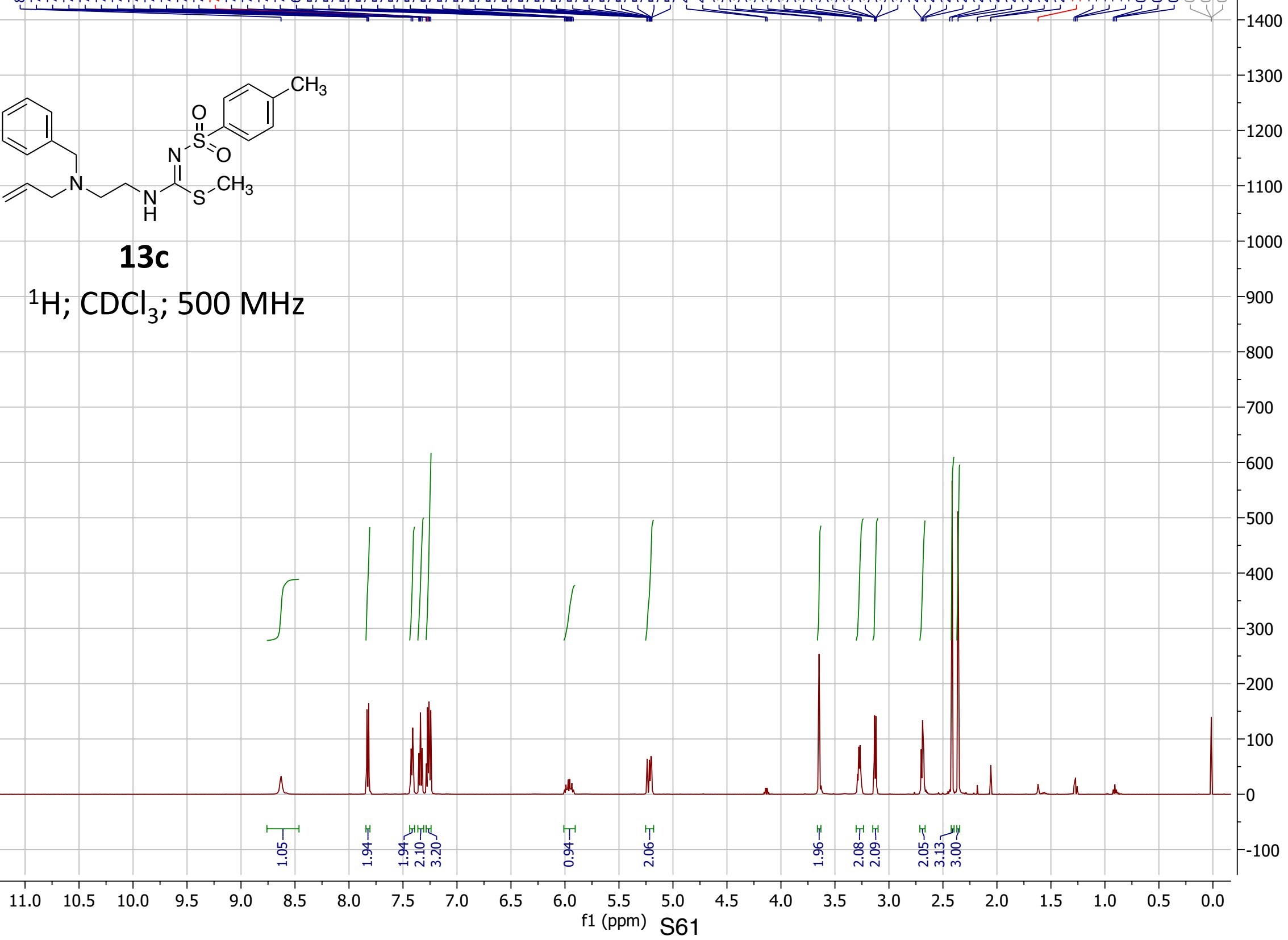




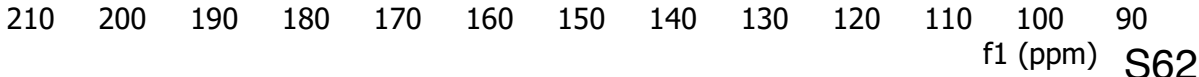


O $\mathrm{CH}_{3}$

$\rightarrow \quad \mathrm{N}^{-\mathrm{S}}=$ O $\underset{\mathrm{N}}{\mathrm{N}} \mathrm{N}_{\mathrm{H}}^{\mathrm{N}} \mathrm{CH}_{3}$

$13 d$

\section{${ }^{1} \mathrm{H} ; \mathrm{CDCl}_{3} ; 500 \mathrm{MHz}$}

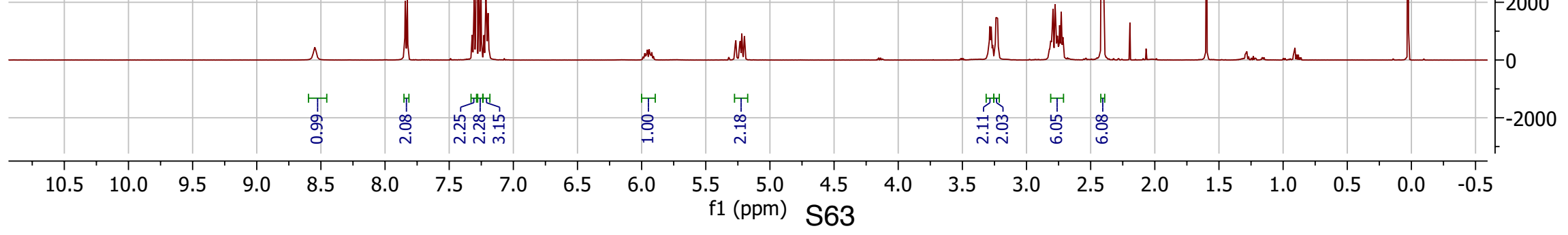


<smiles>C=CCN(CCNC(=NS(=O)(=O)c1ccc(C)cc1)SC)Cc1cccc(Br)c1</smiles>

\section{$13 e$}

\section{${ }^{1} \mathrm{H} ; \mathrm{CDCl}_{3} ; 500 \mathrm{MHz}$}

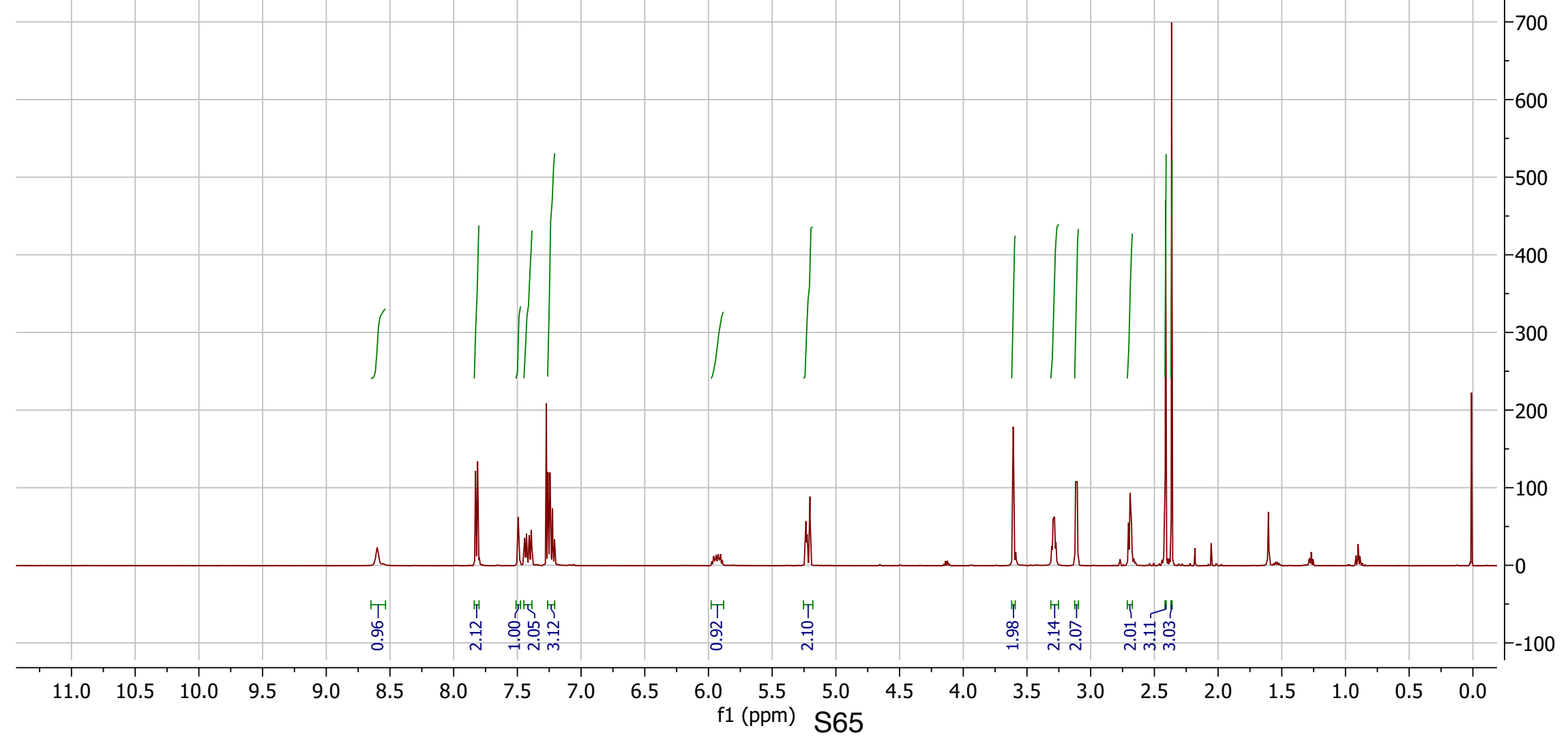



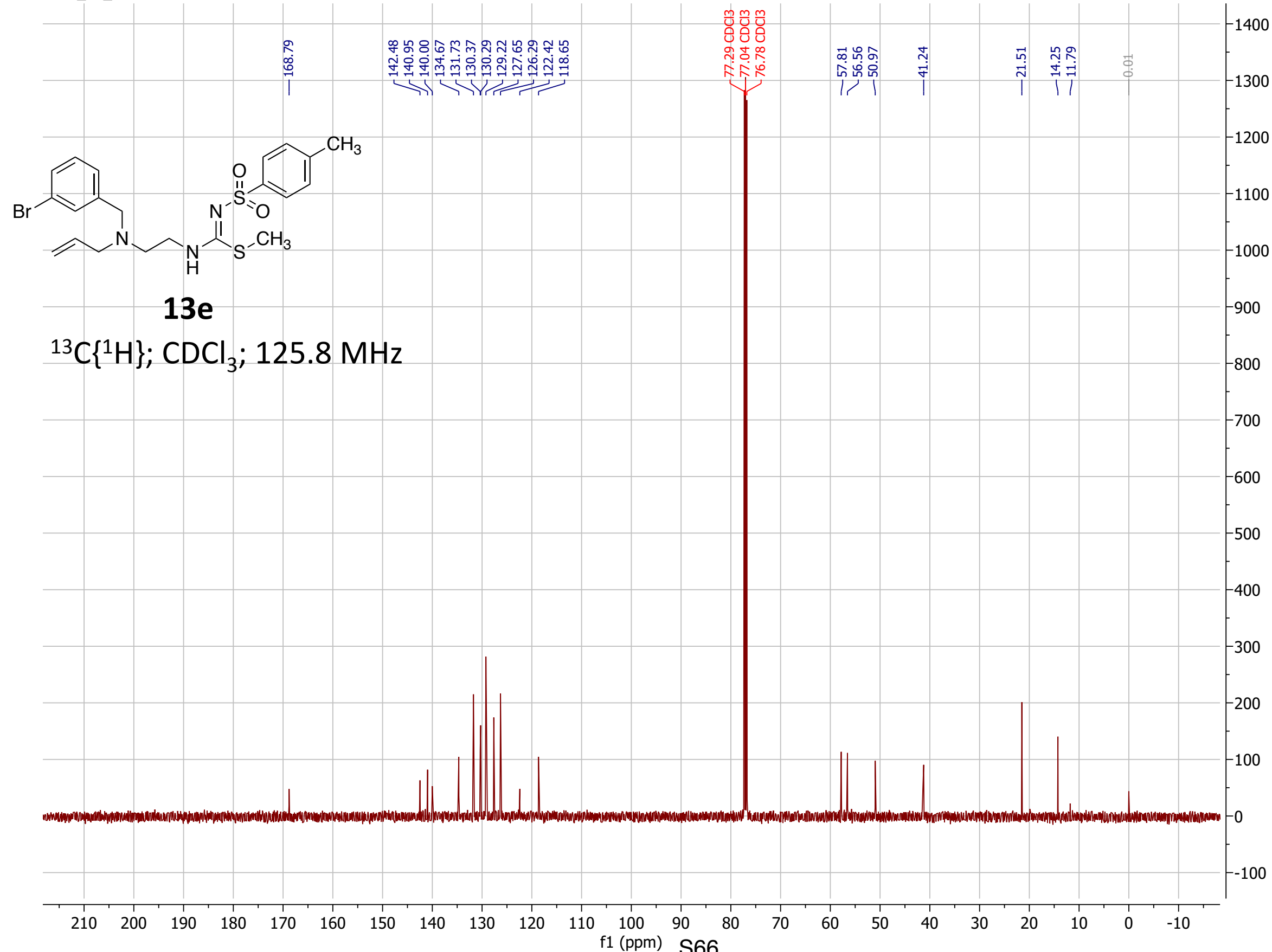


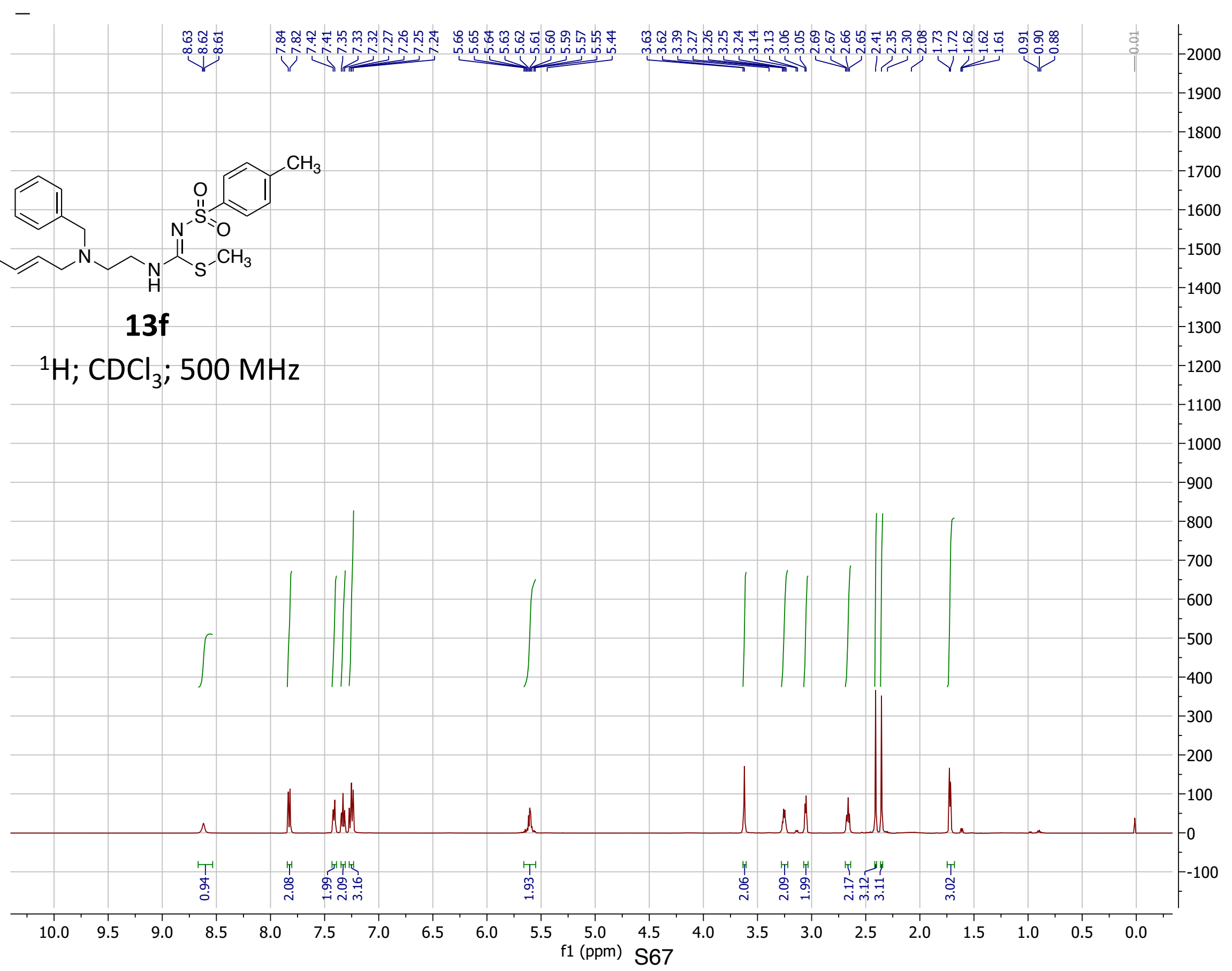



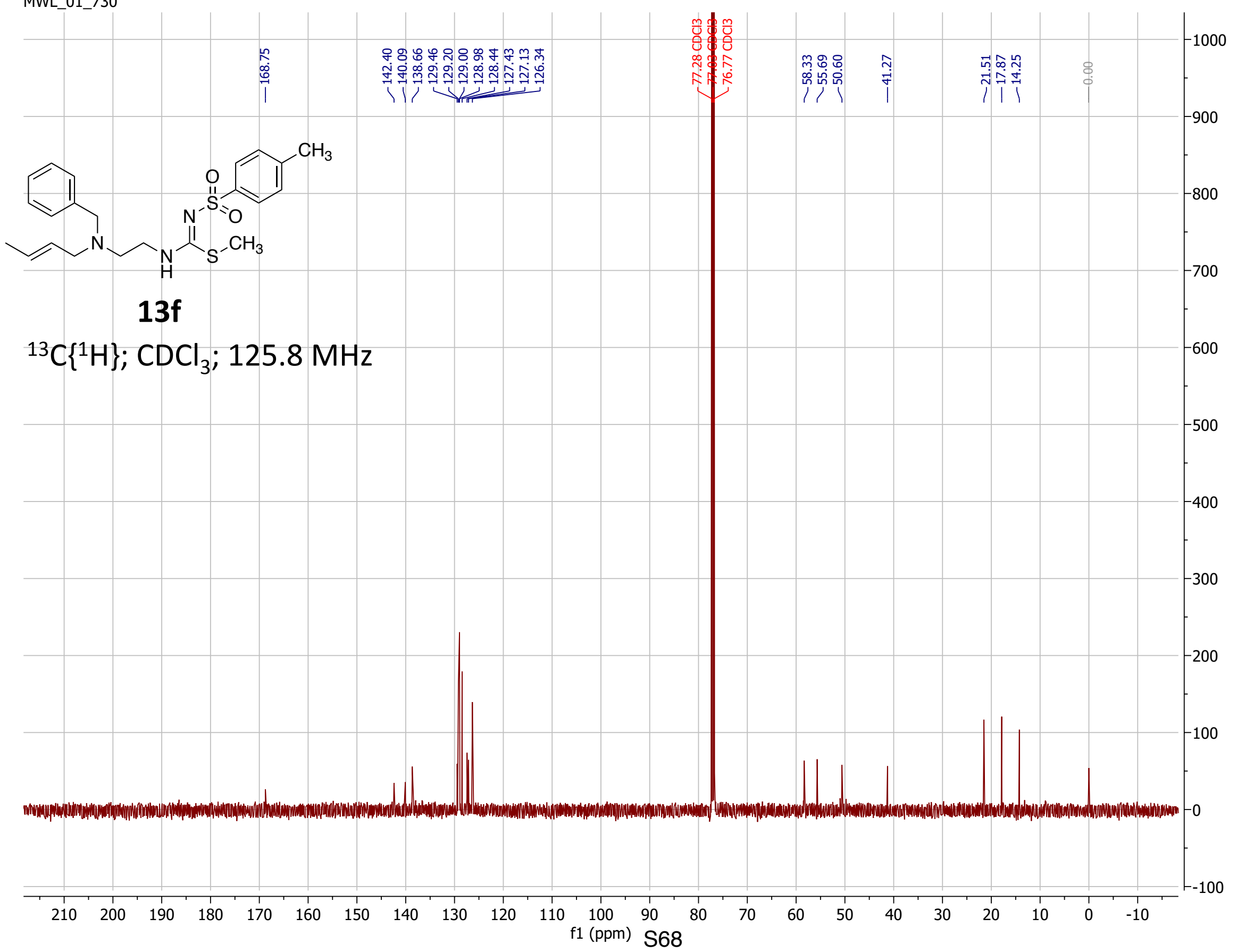


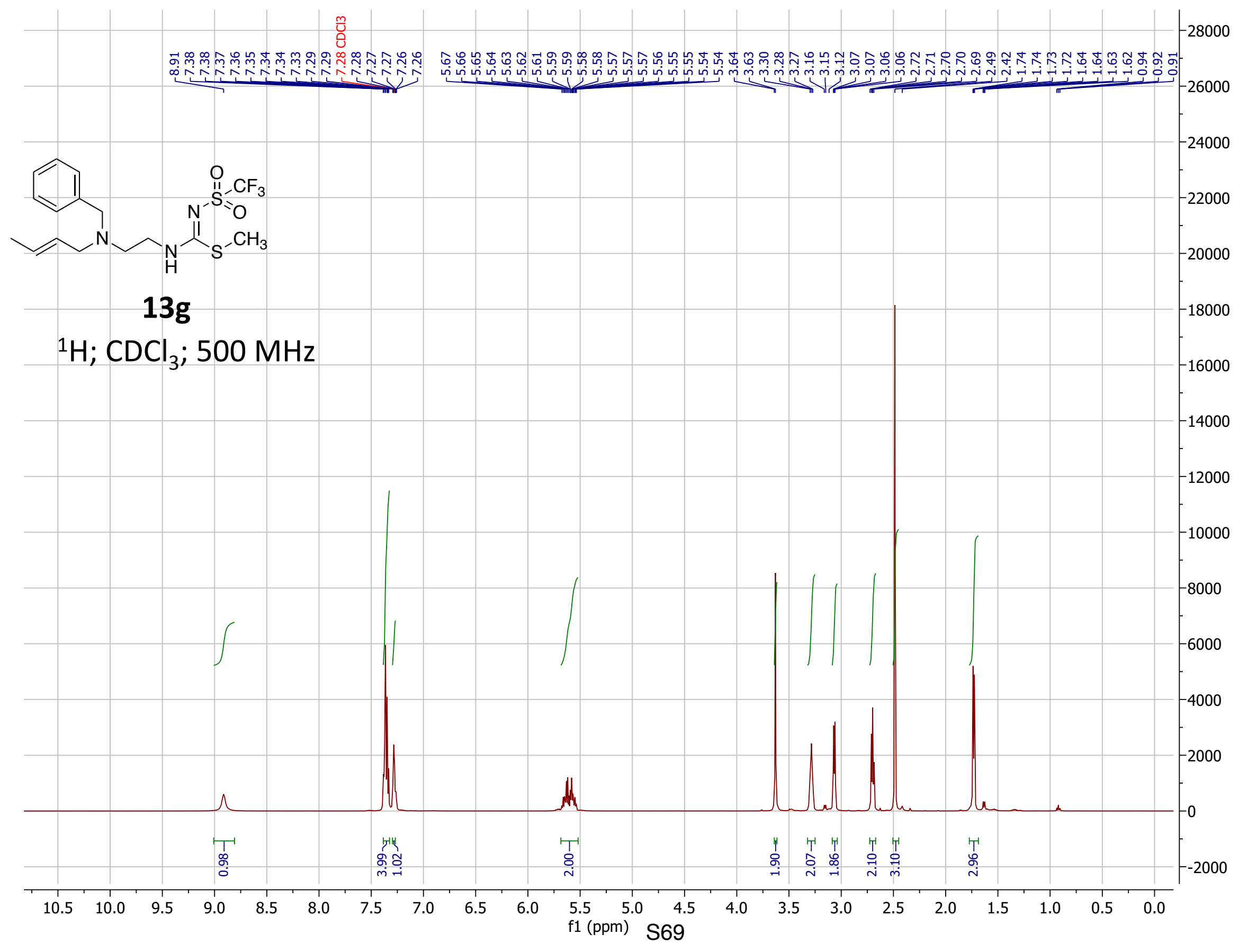




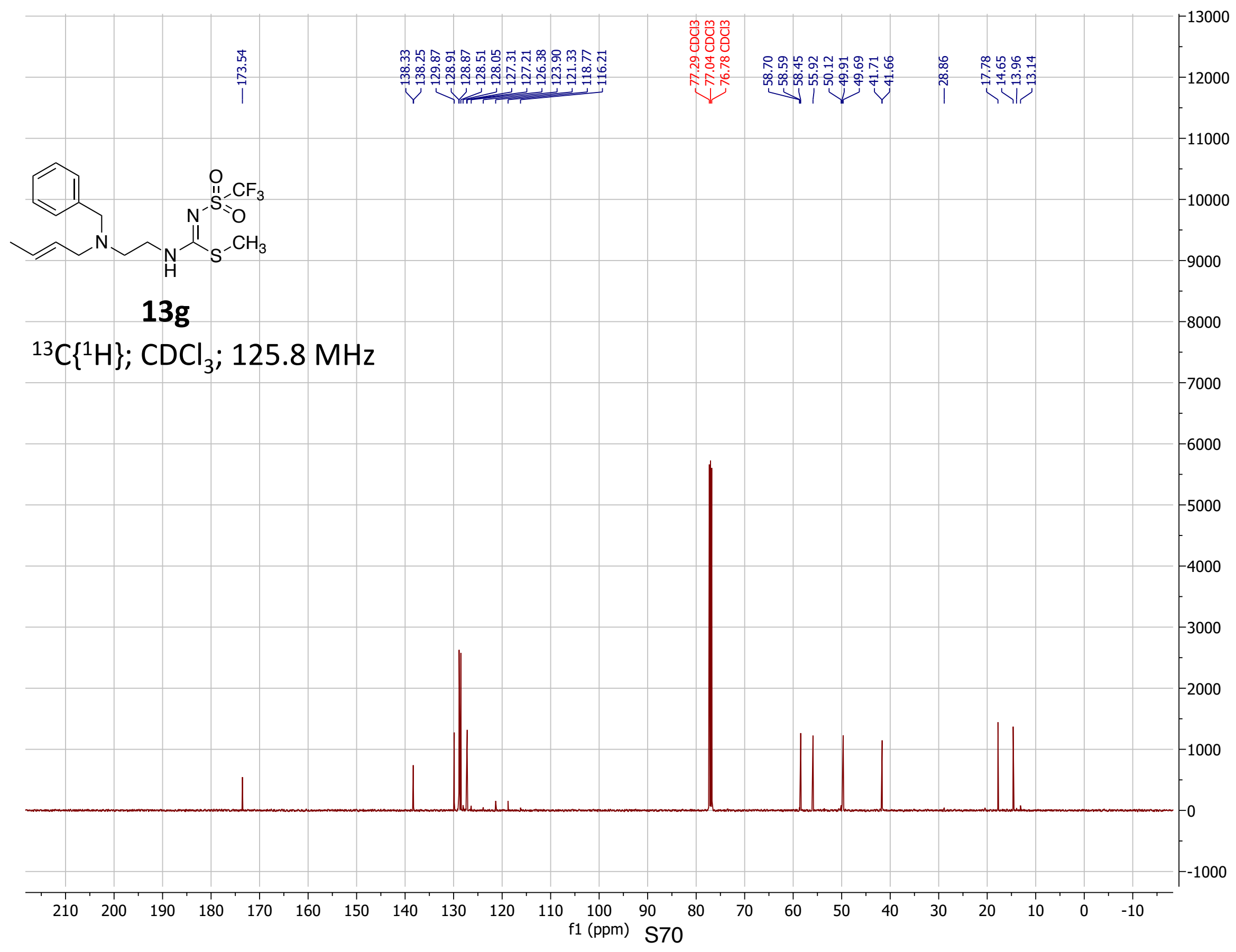




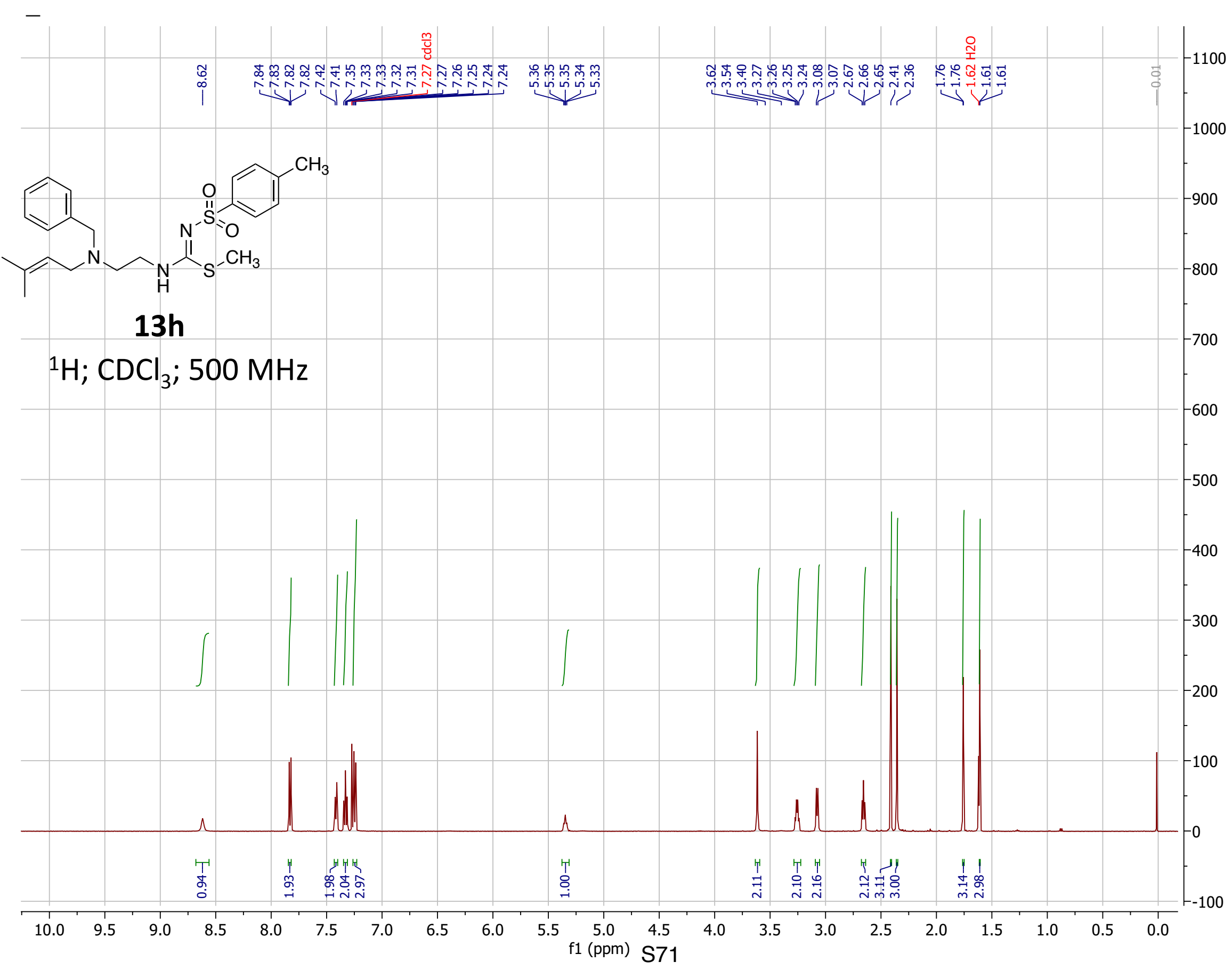




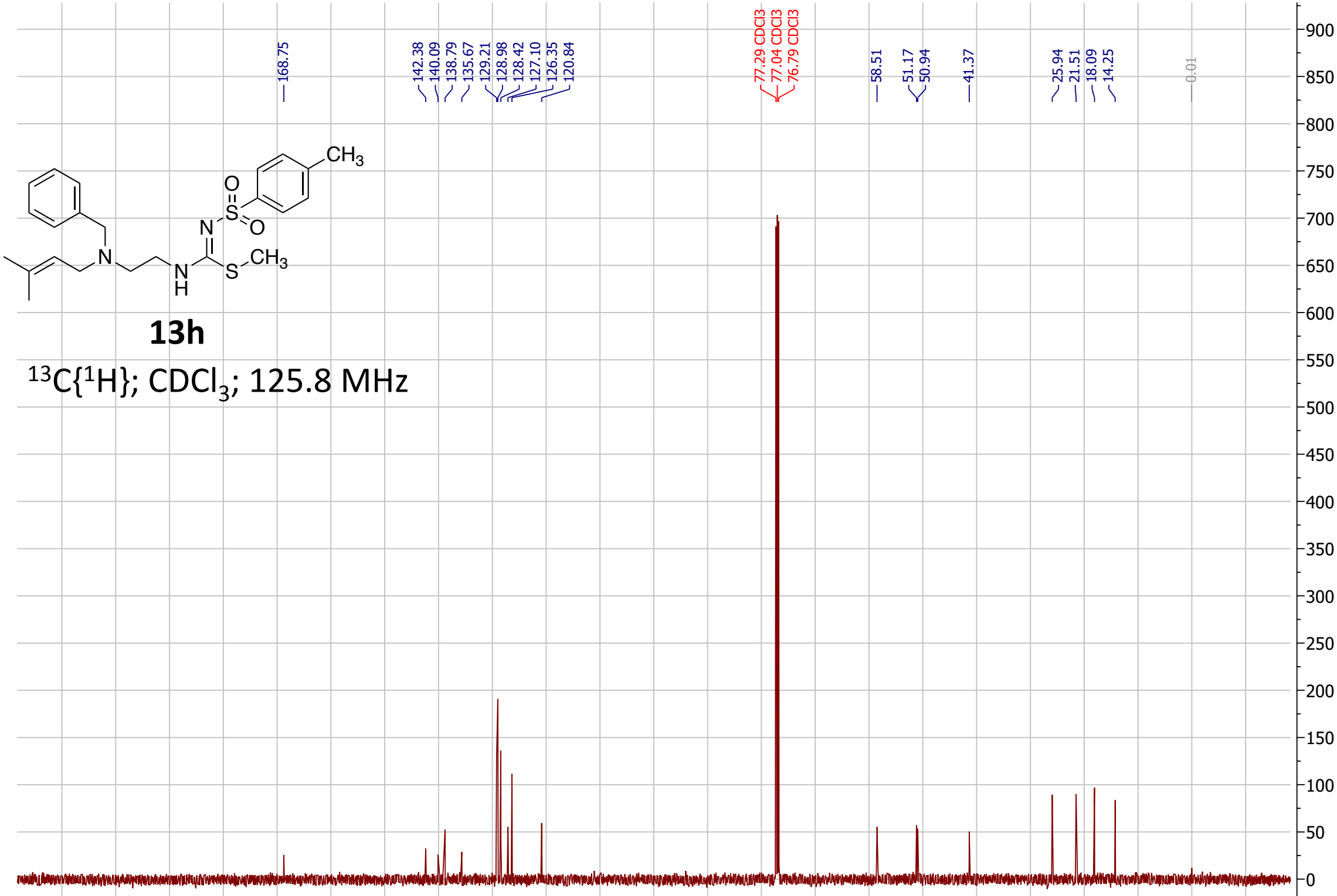




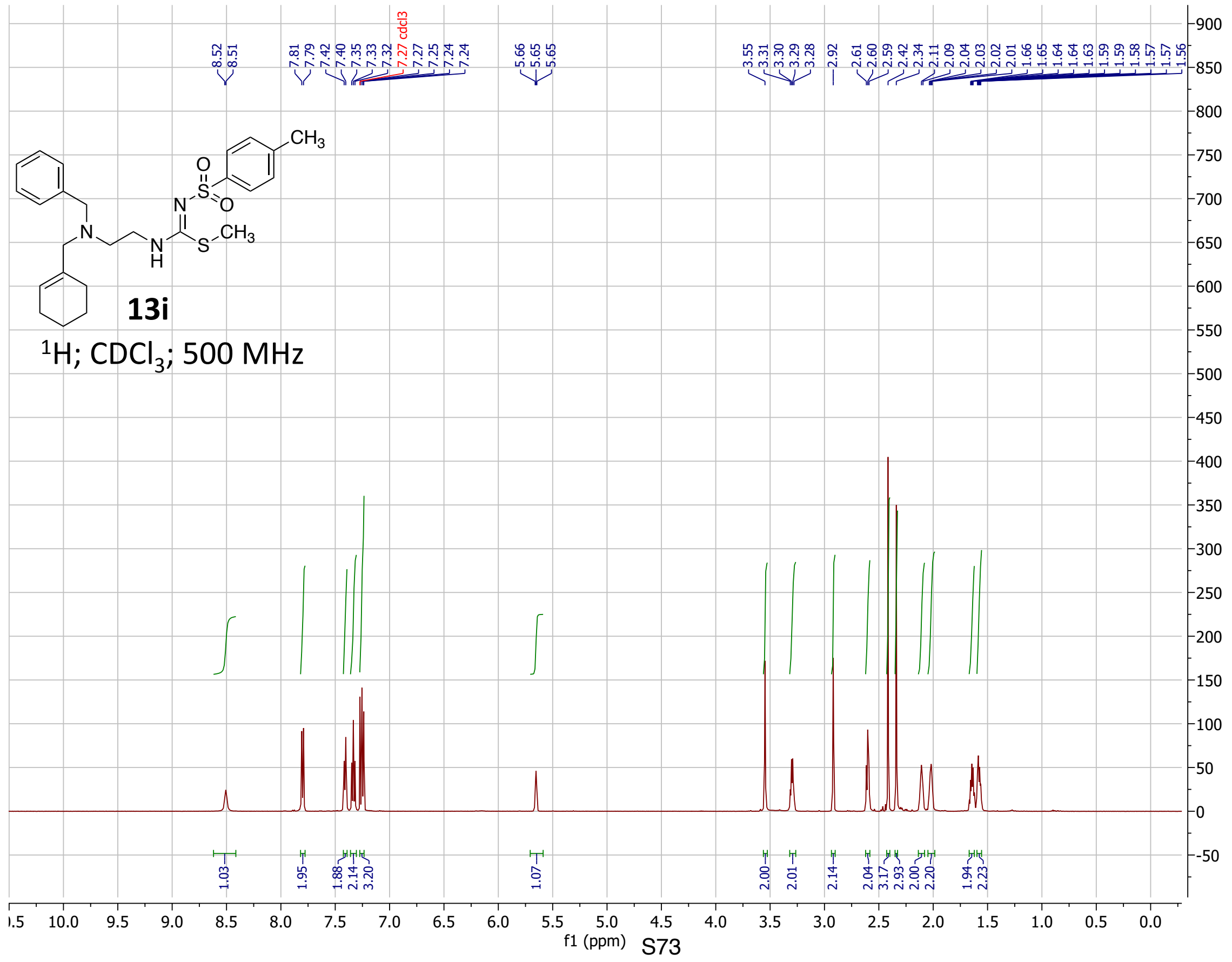




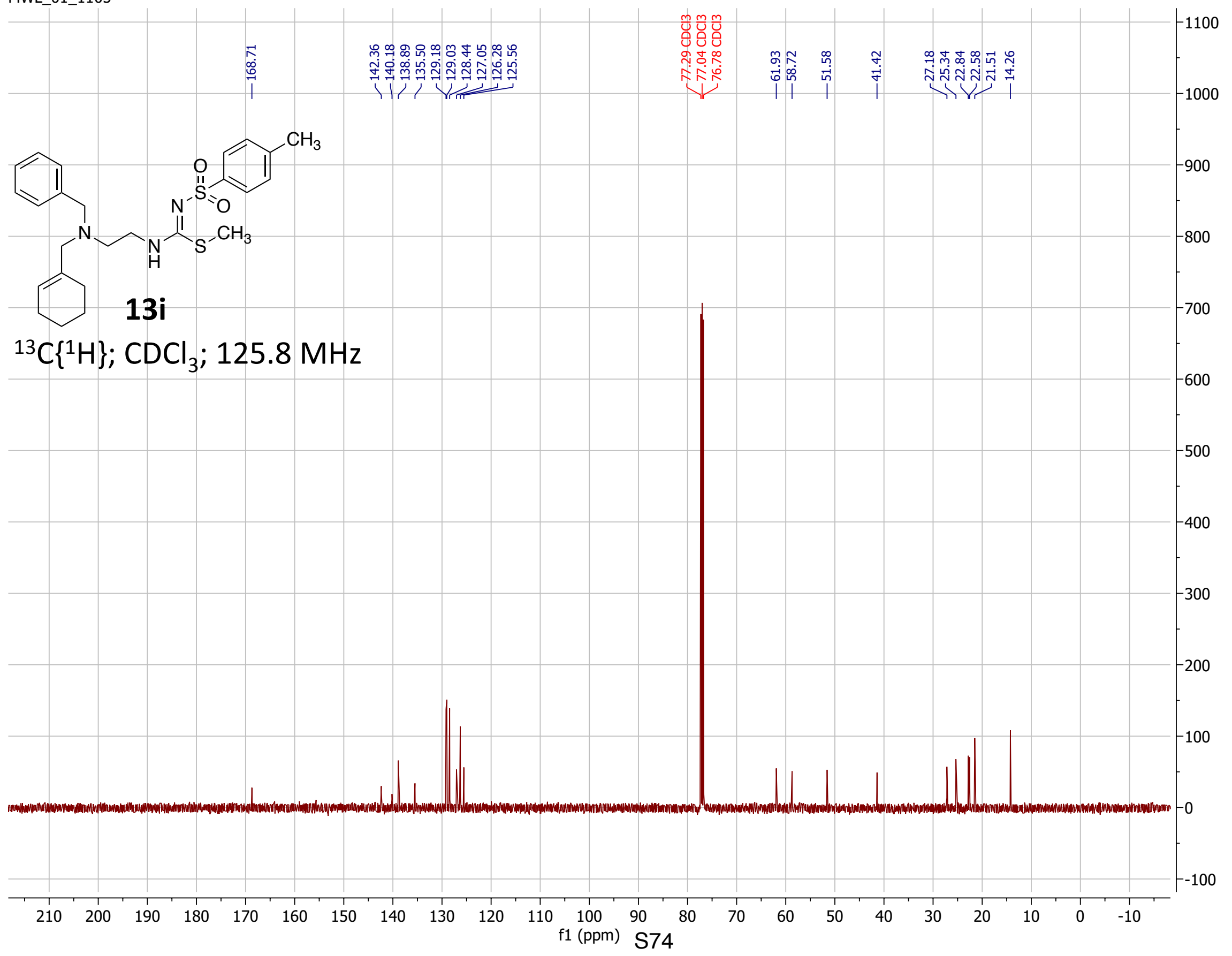


$7 \mathrm{CH}^{\mathrm{CH}_{3}}$

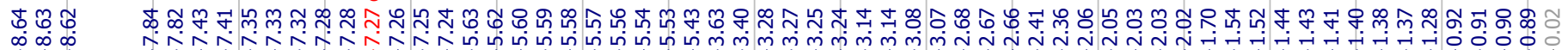

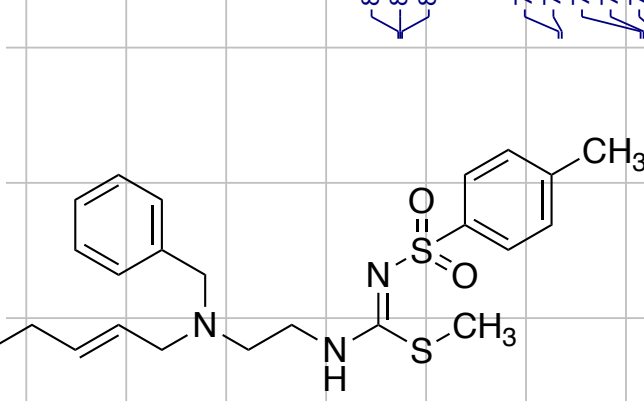

13j

${ }^{1} \mathrm{H} ; \mathrm{CDCl}_{3} ; 500 \mathrm{MHz}$

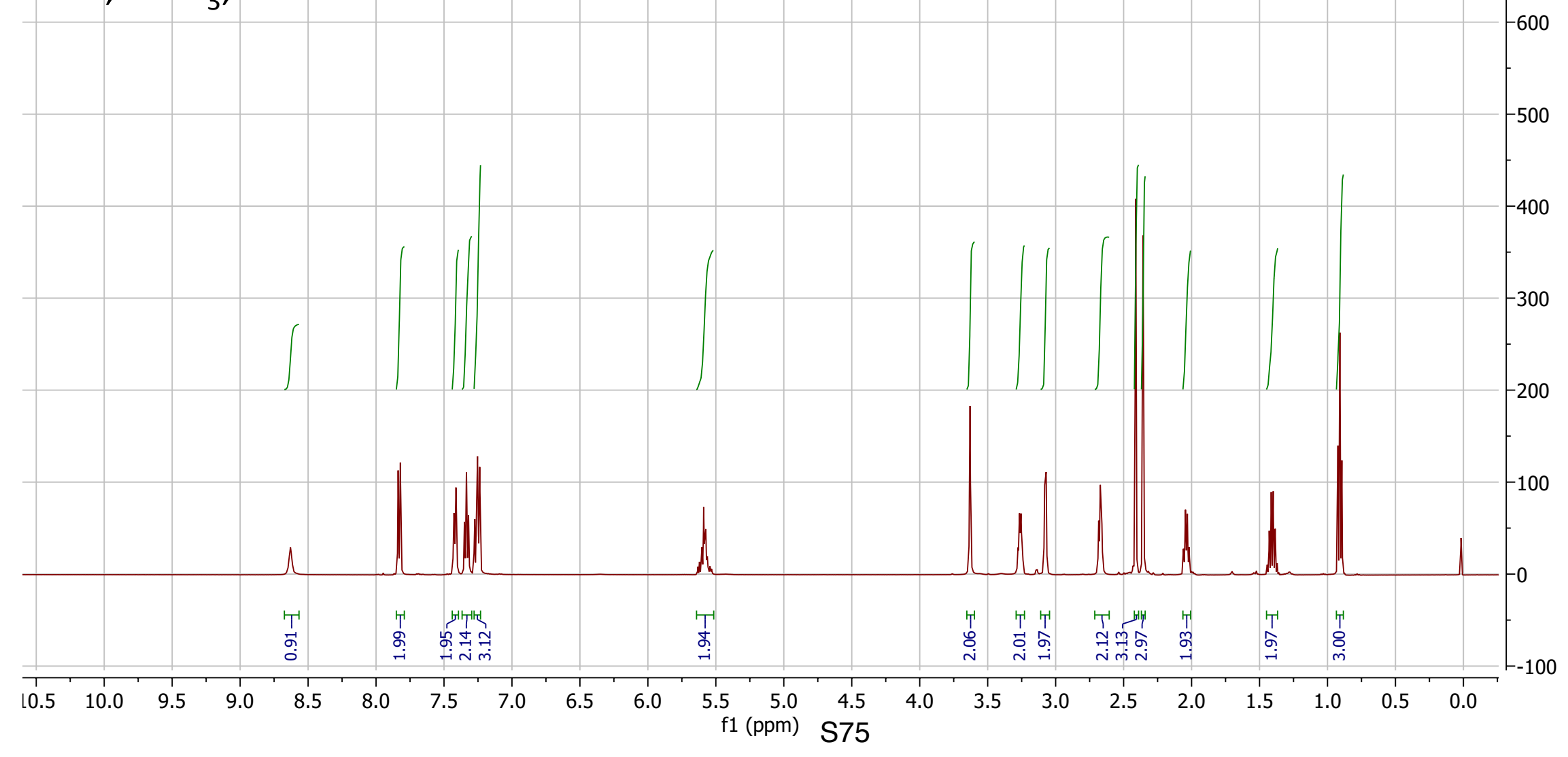



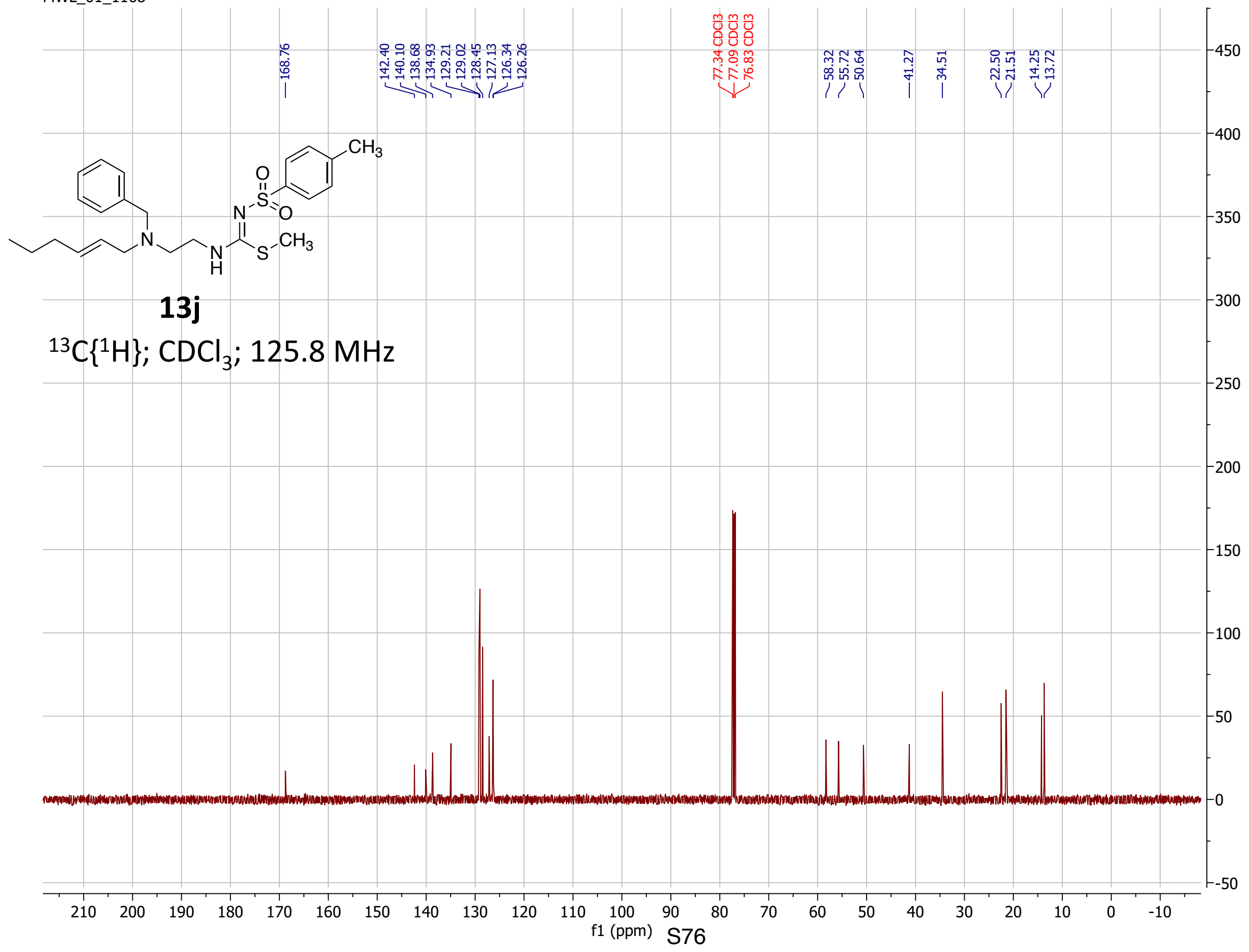


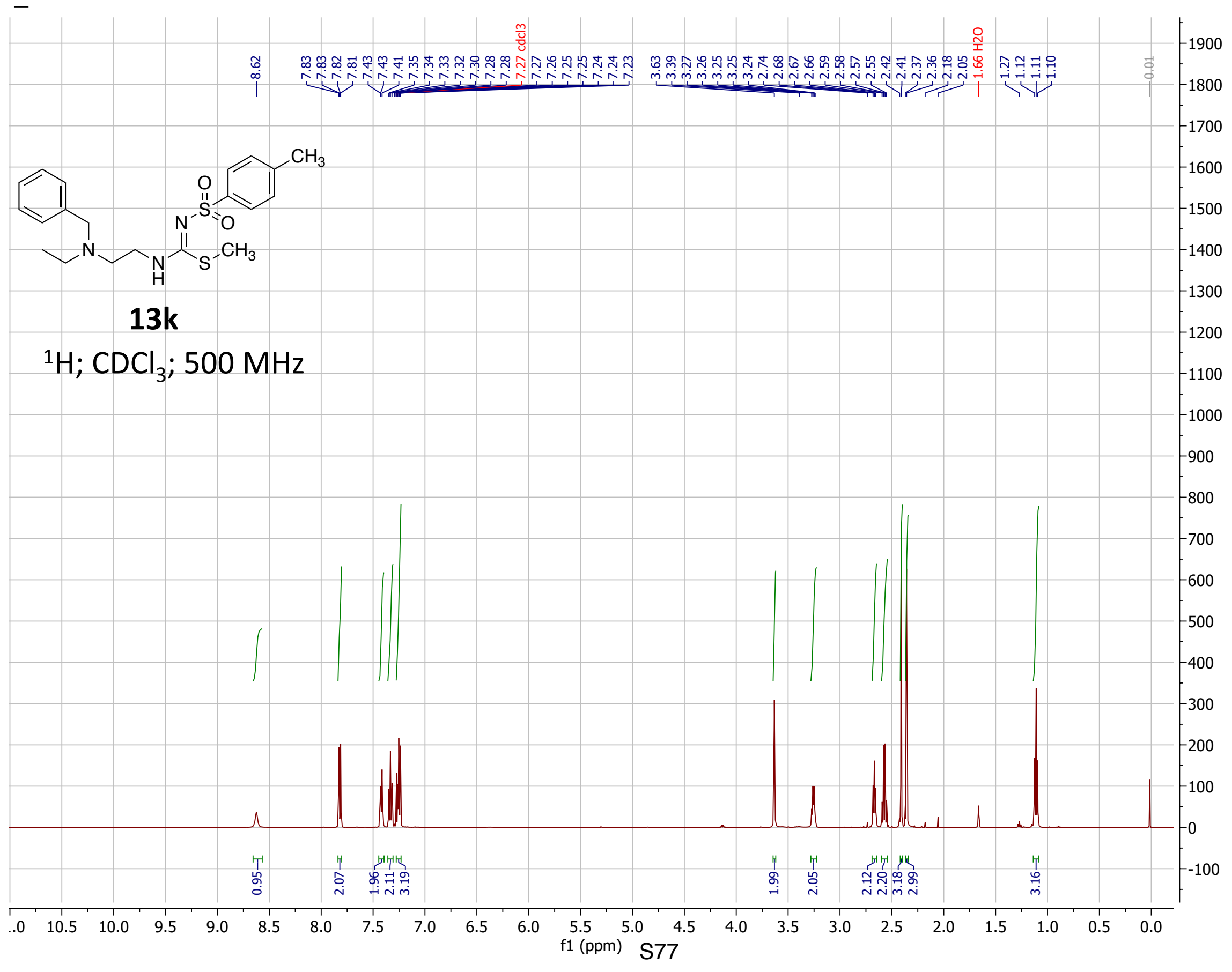




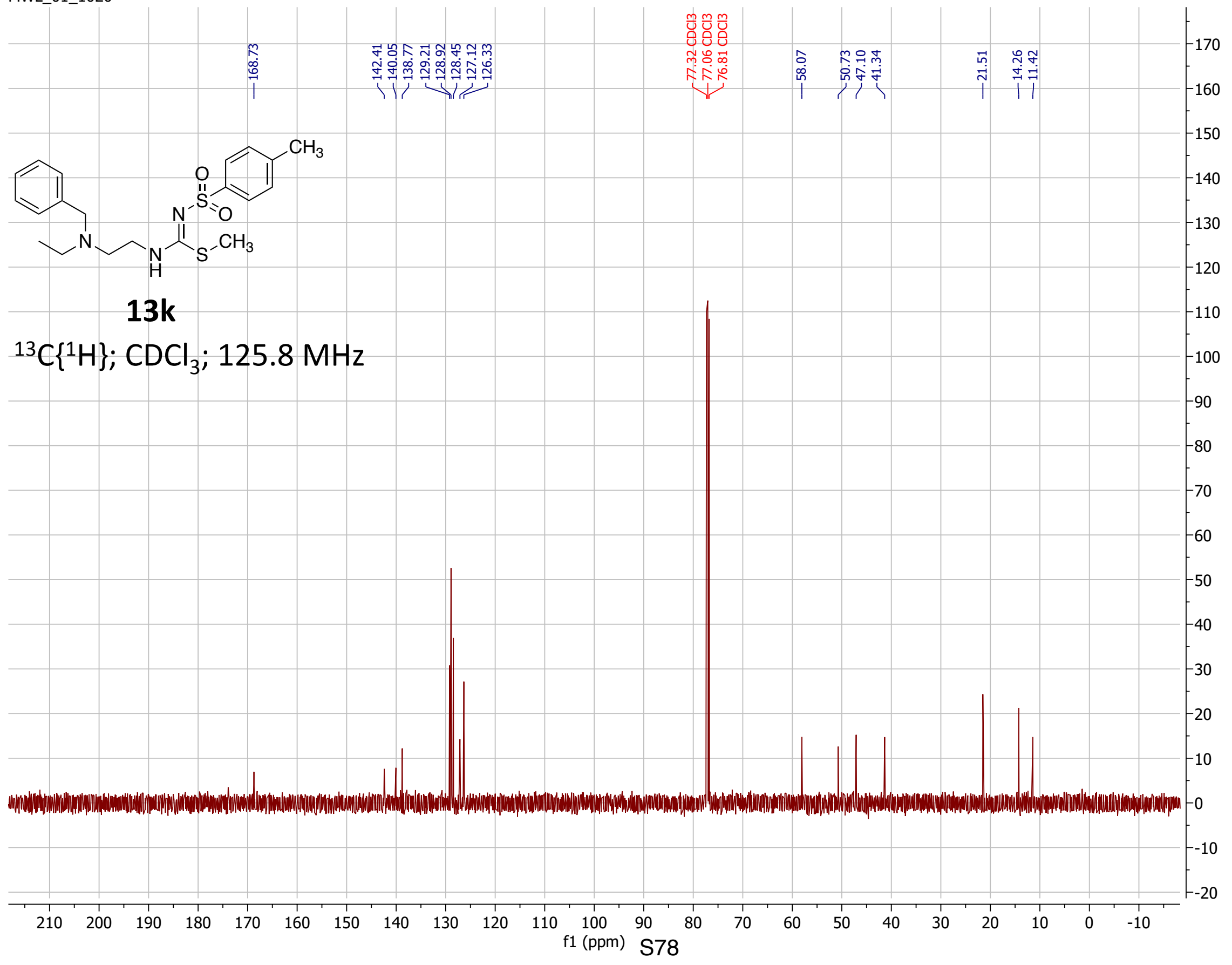




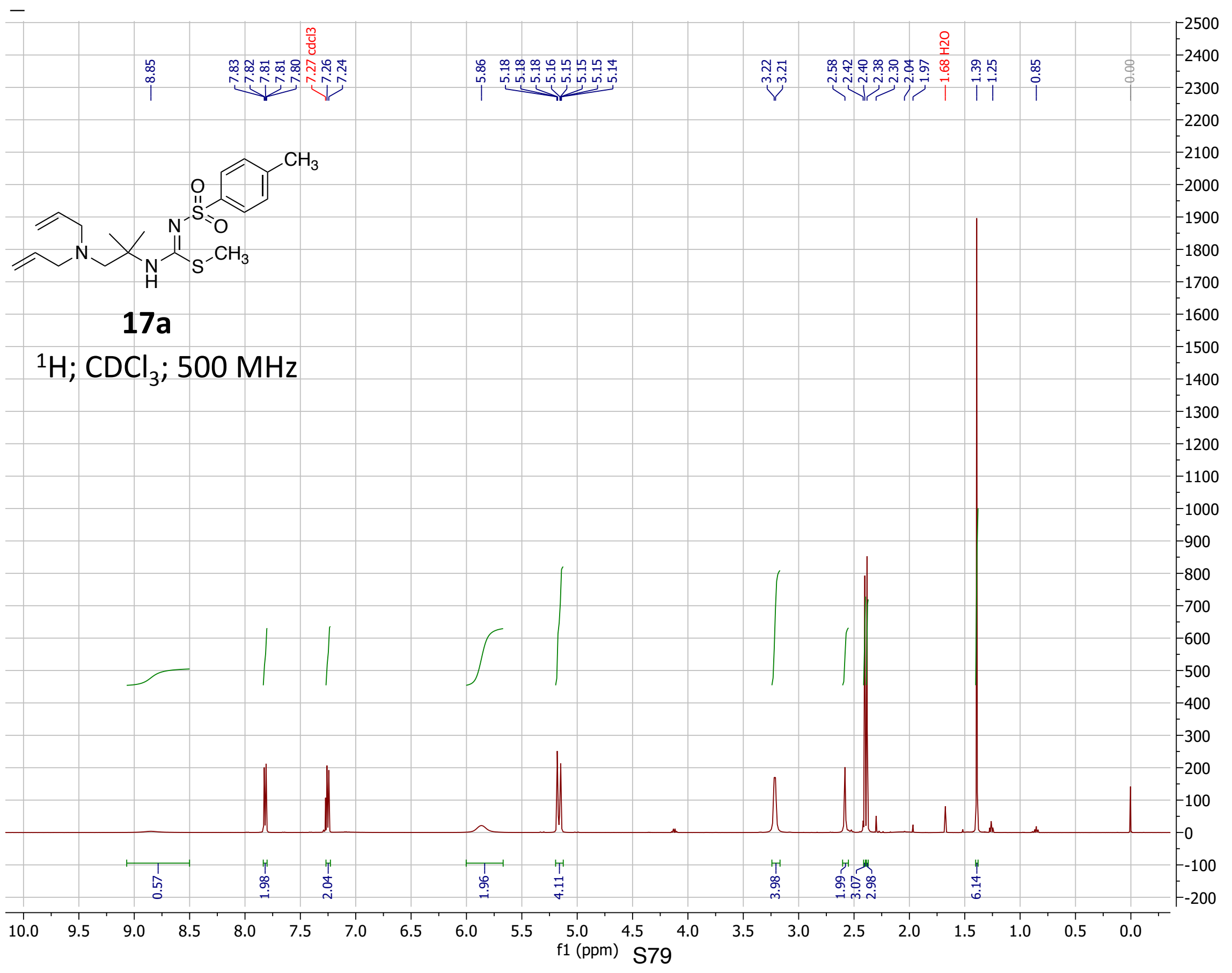




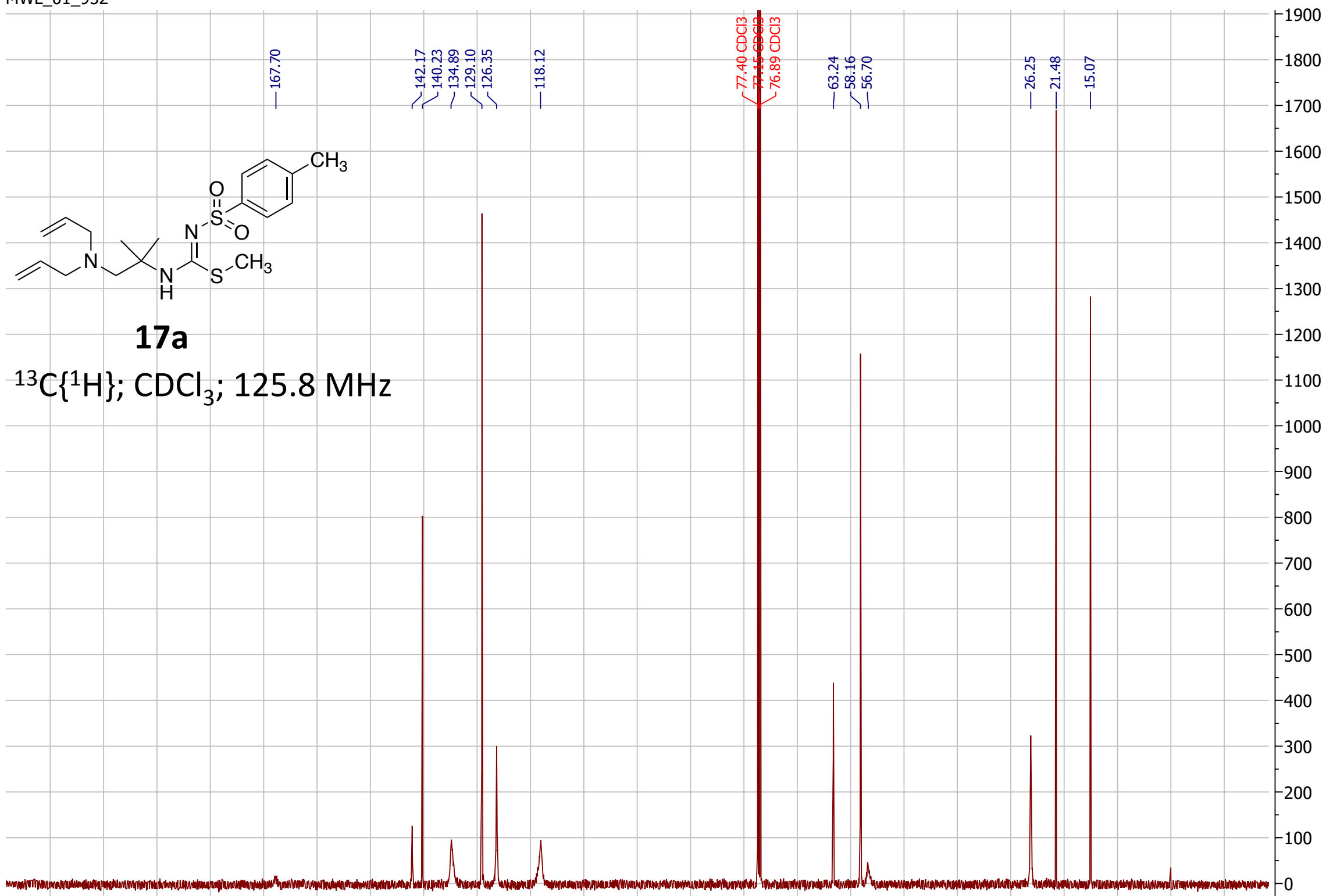




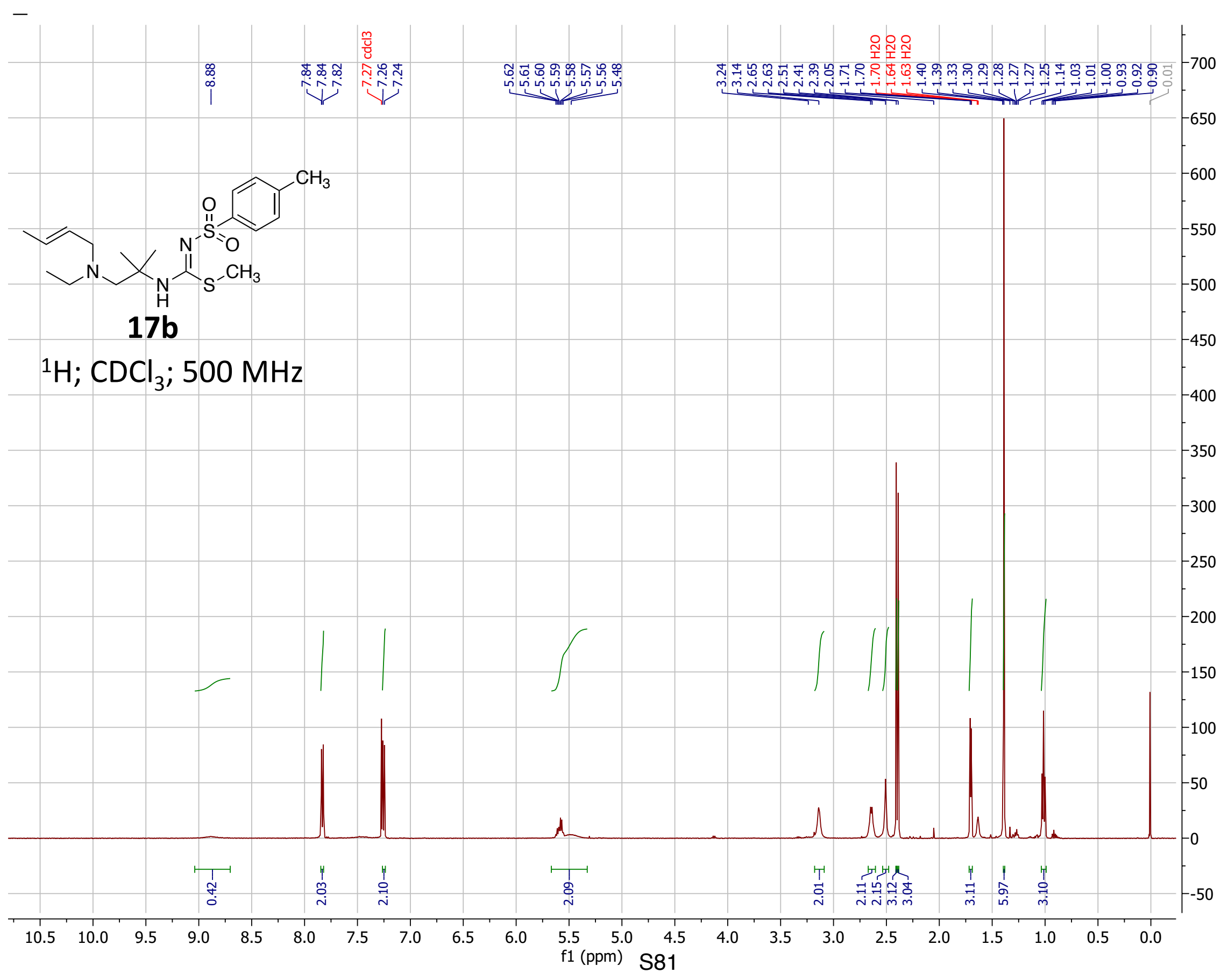




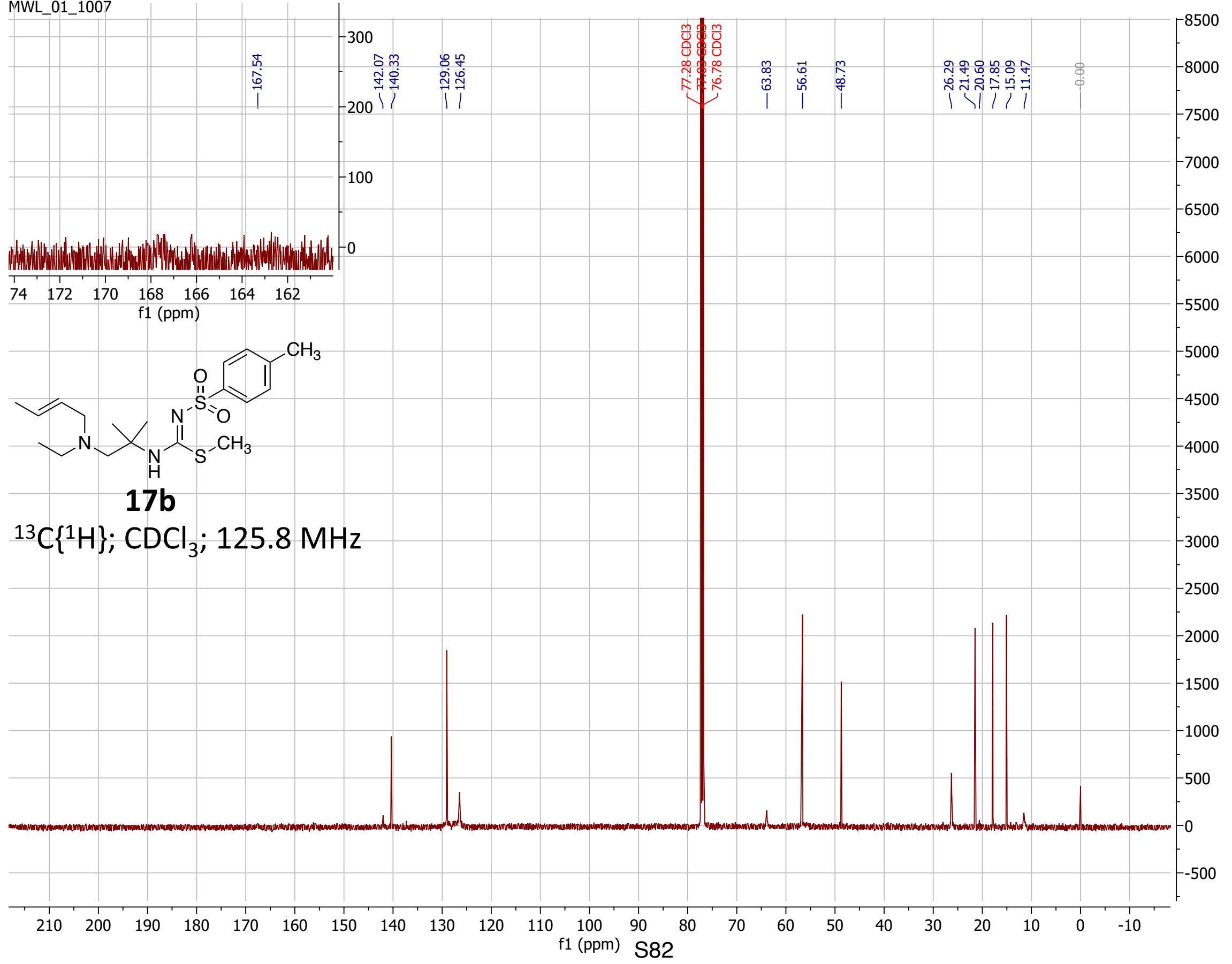


0 $\mathrm{CH}_{3}$<smiles>C=CCN(CC=C)C(C)(C)CNC(=NS(=O)C=CCC)SC</smiles>

20

\section{${ }^{1} \mathrm{H} ; \mathrm{CDCl}_{3} ; 500 \mathrm{MHz}$}

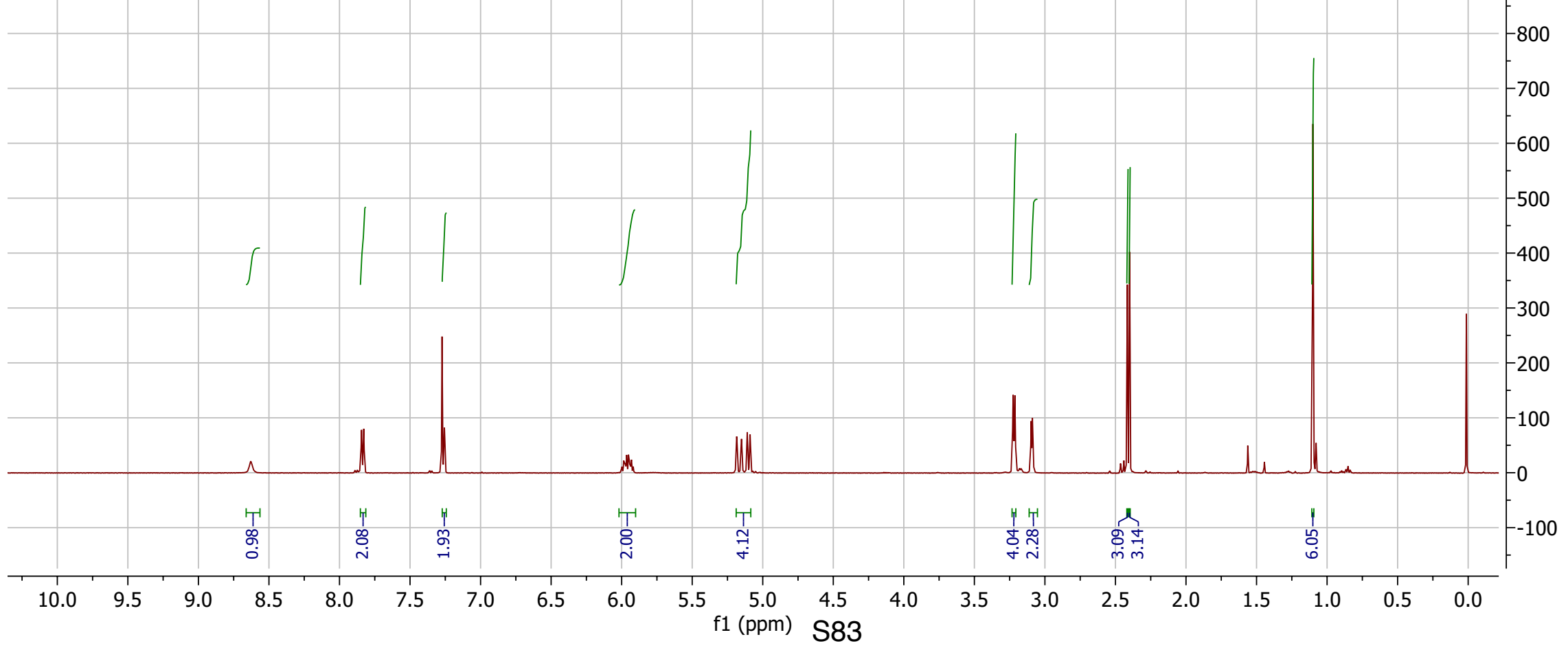



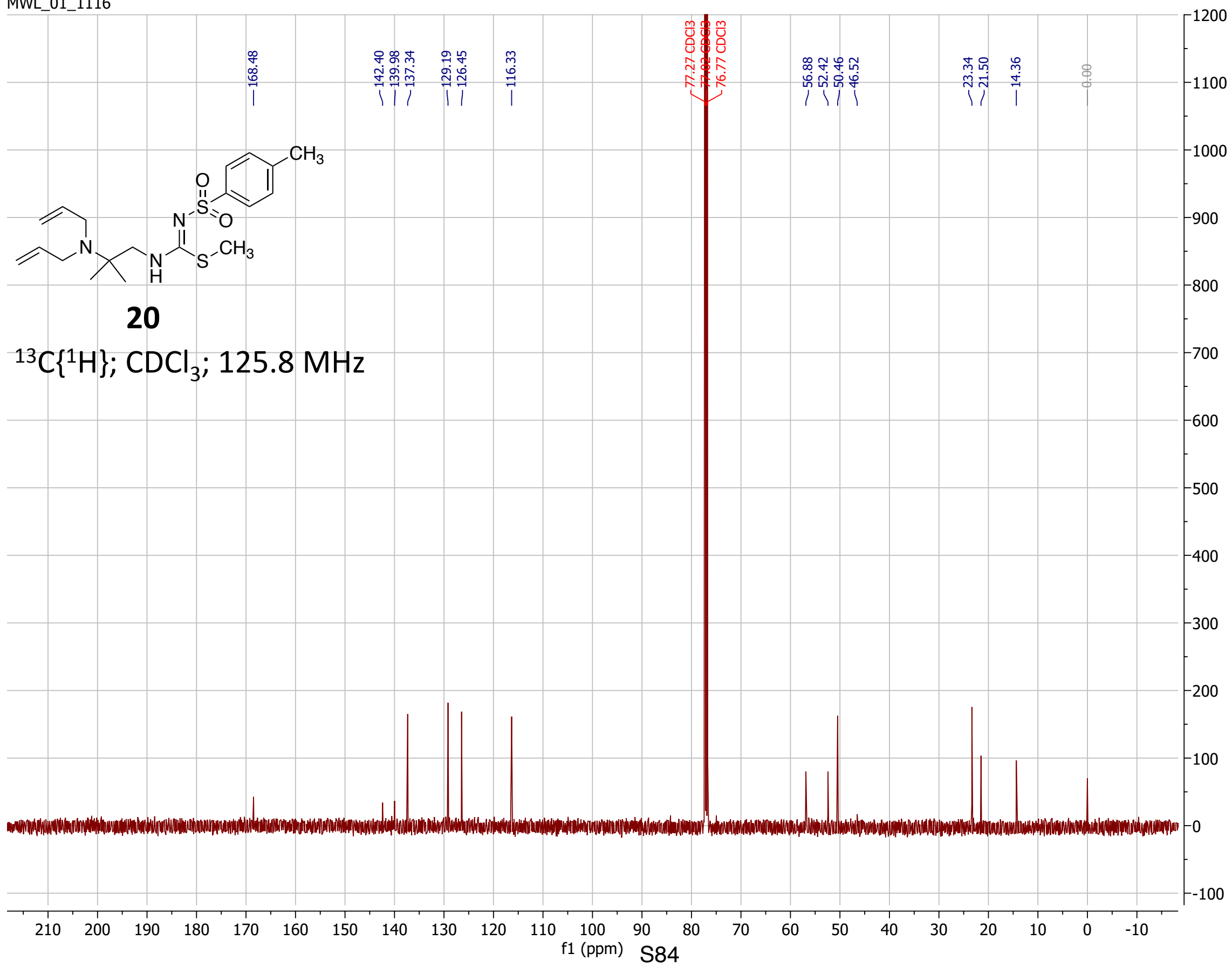


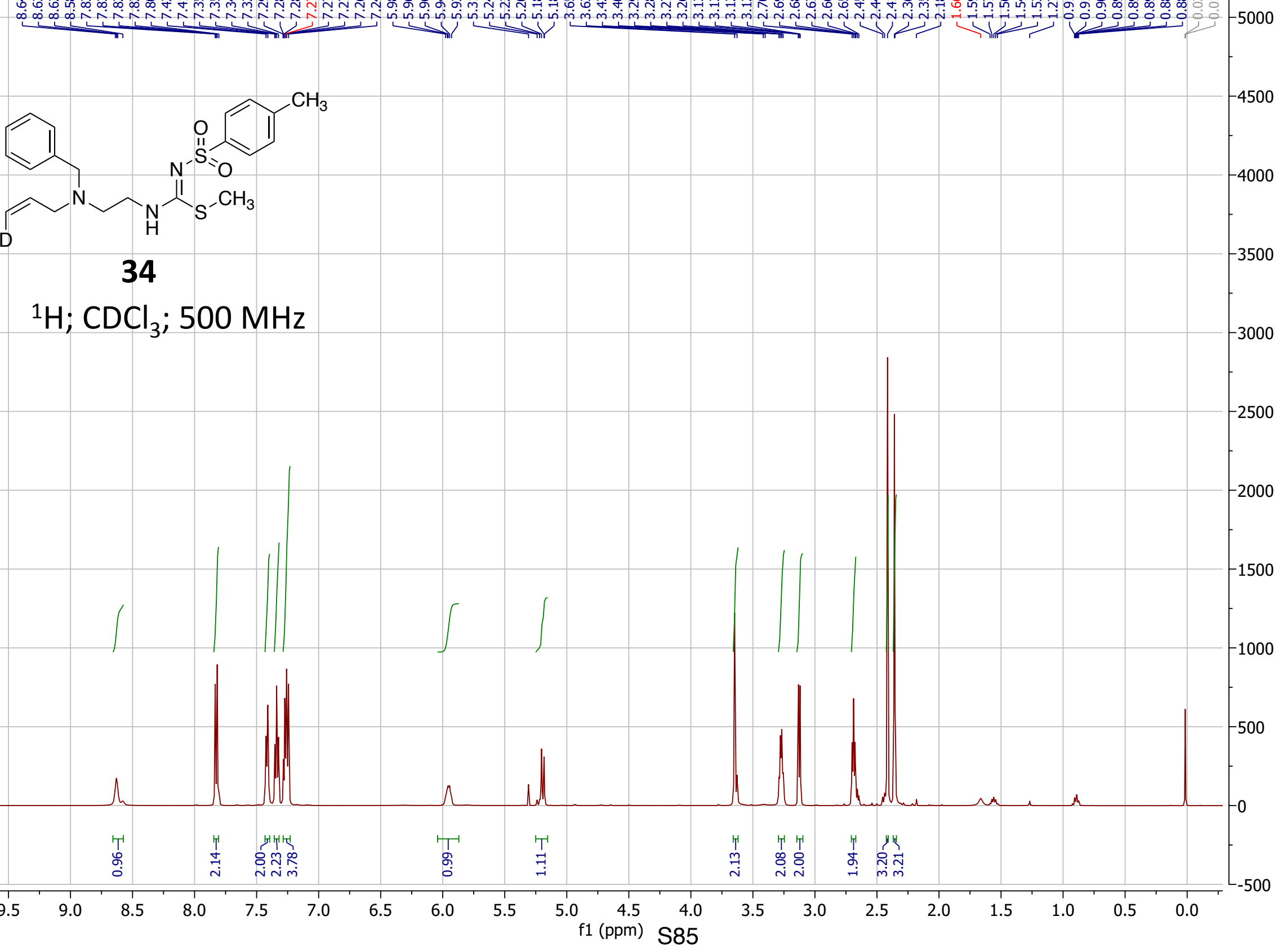




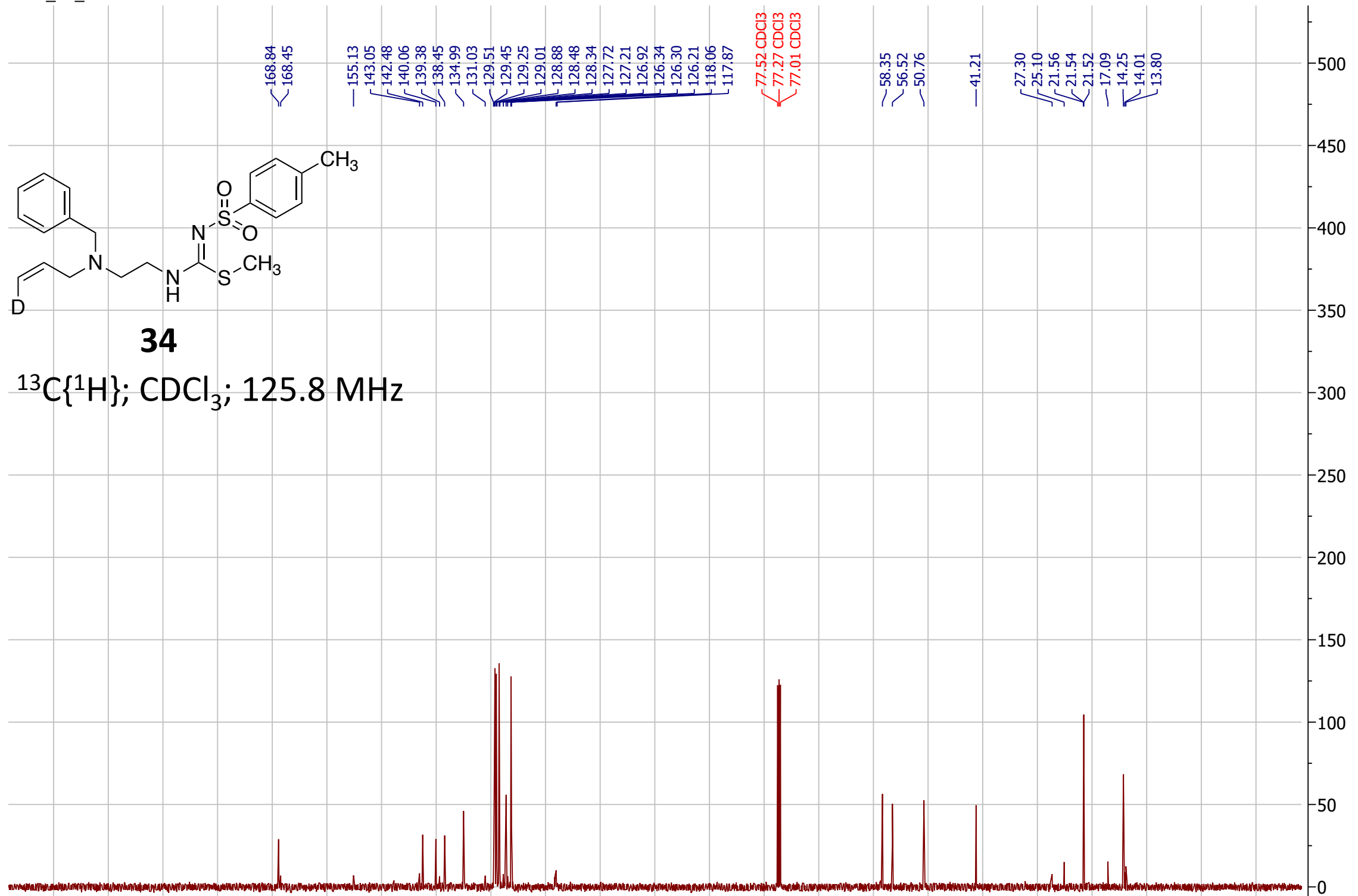




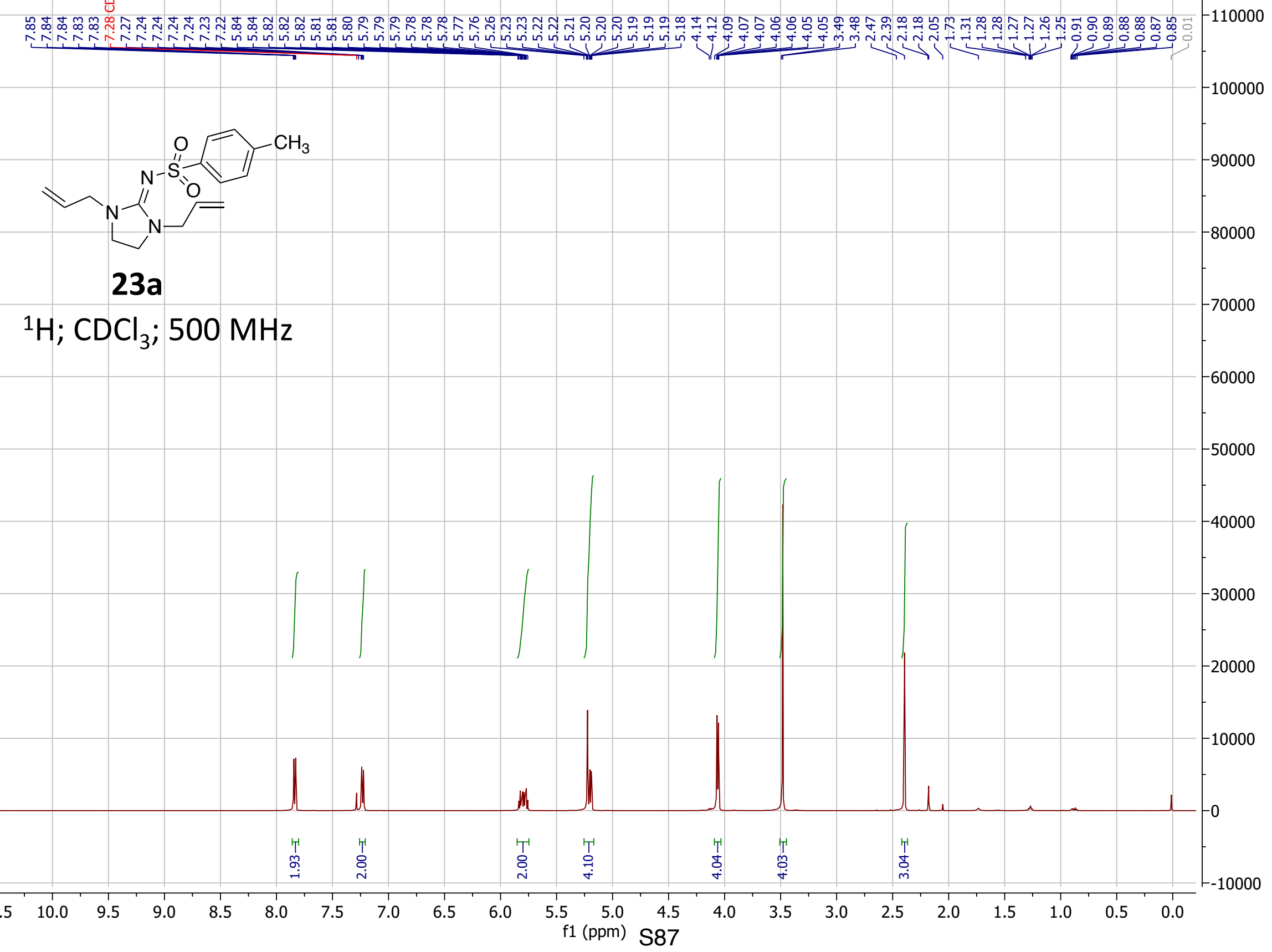




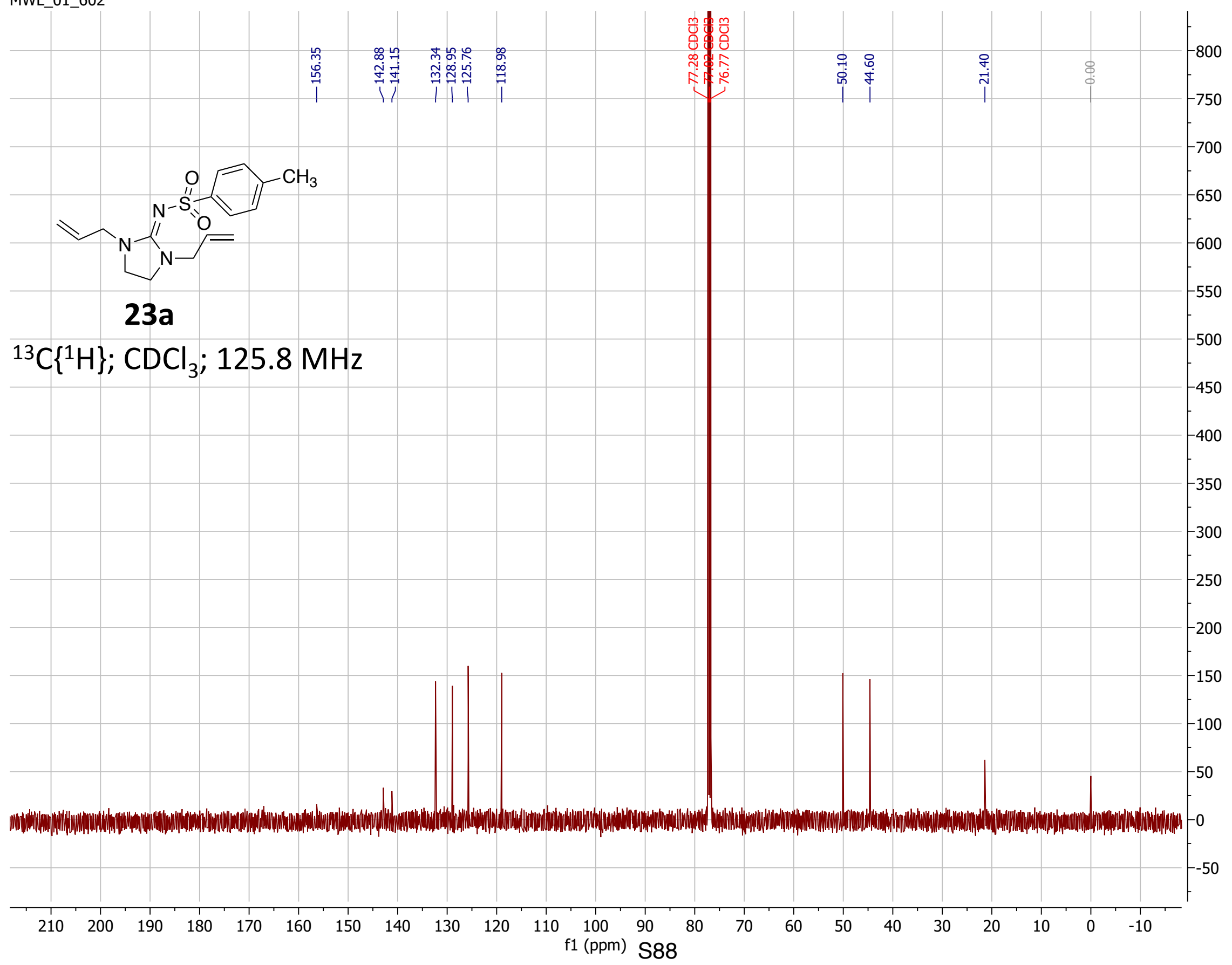




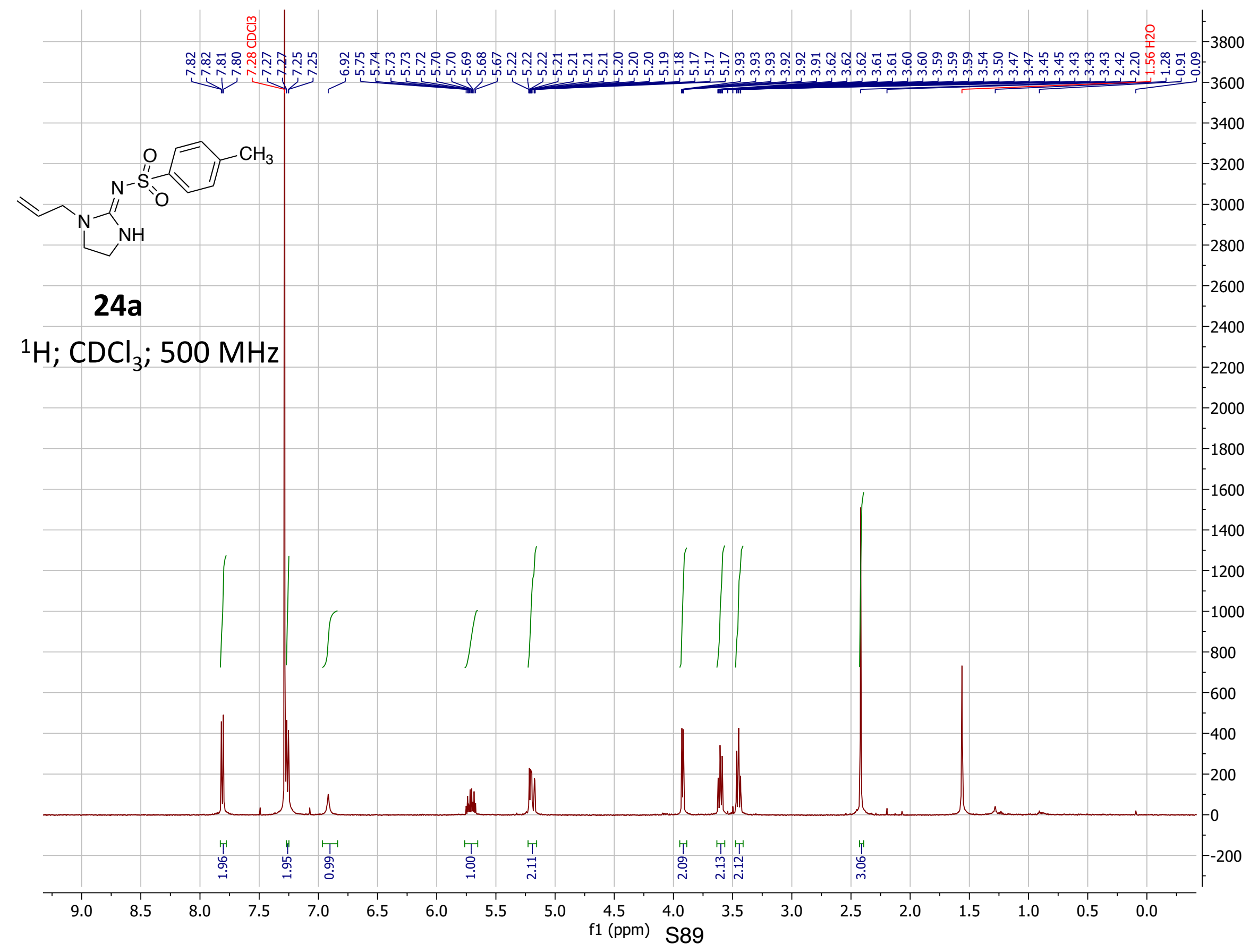




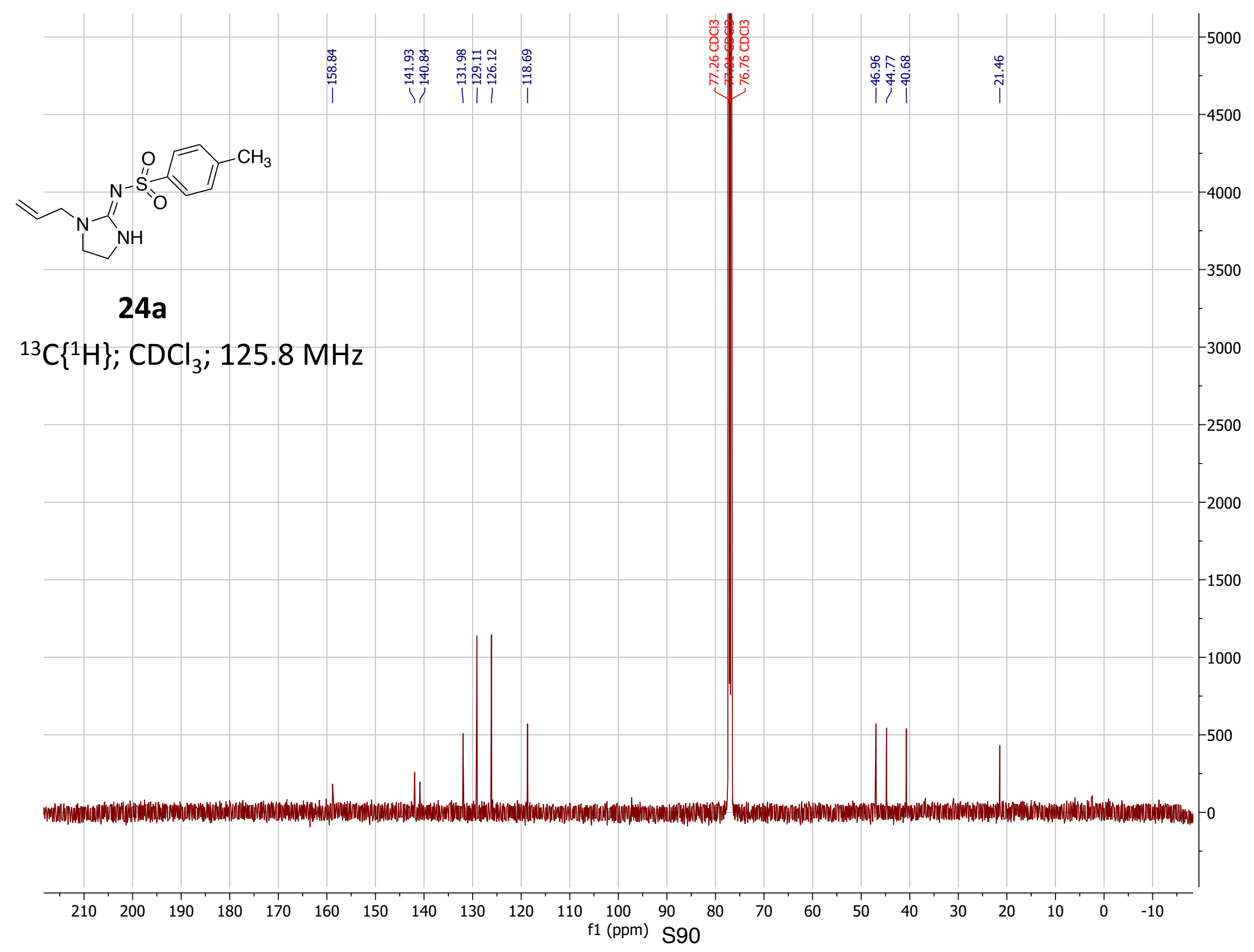




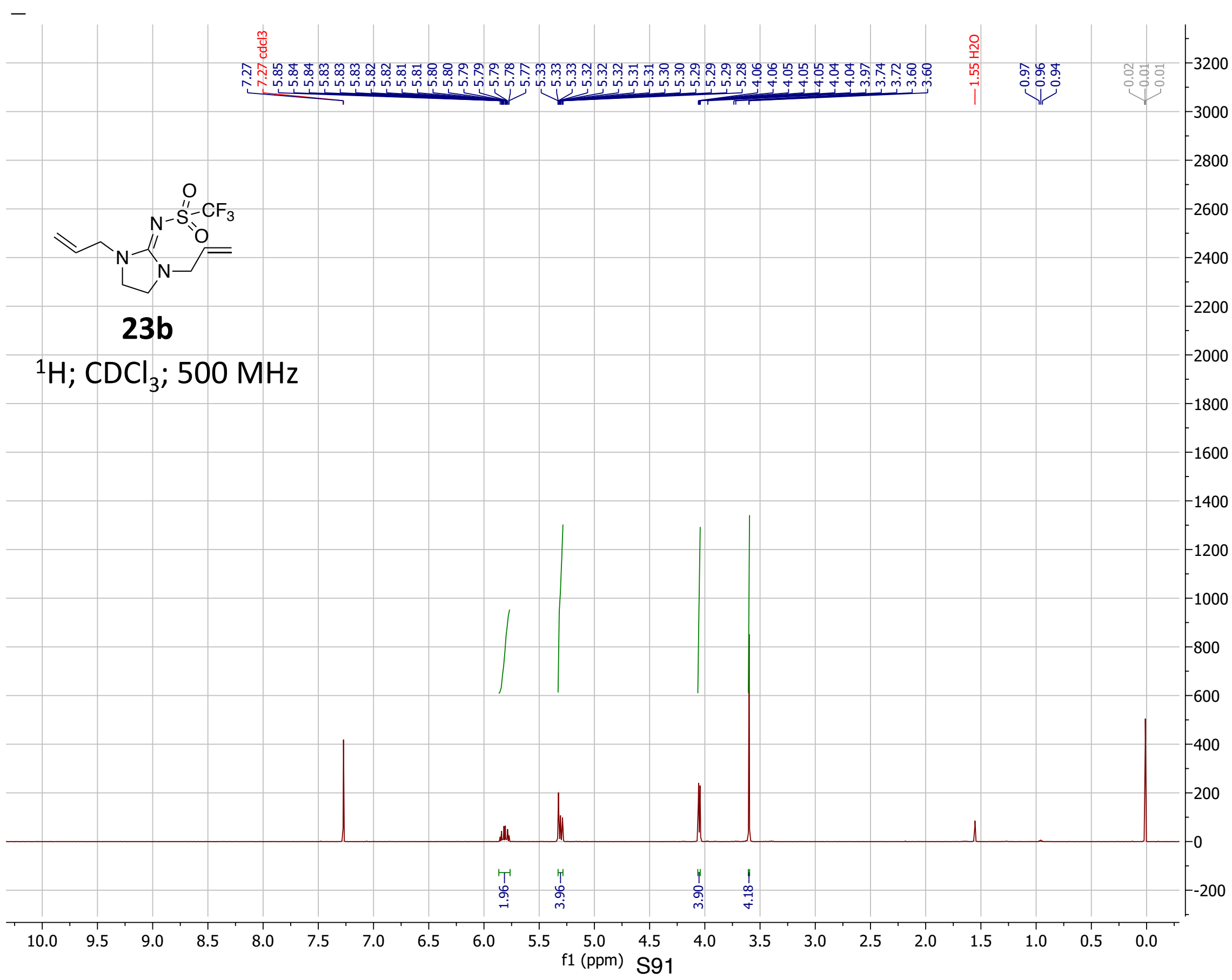




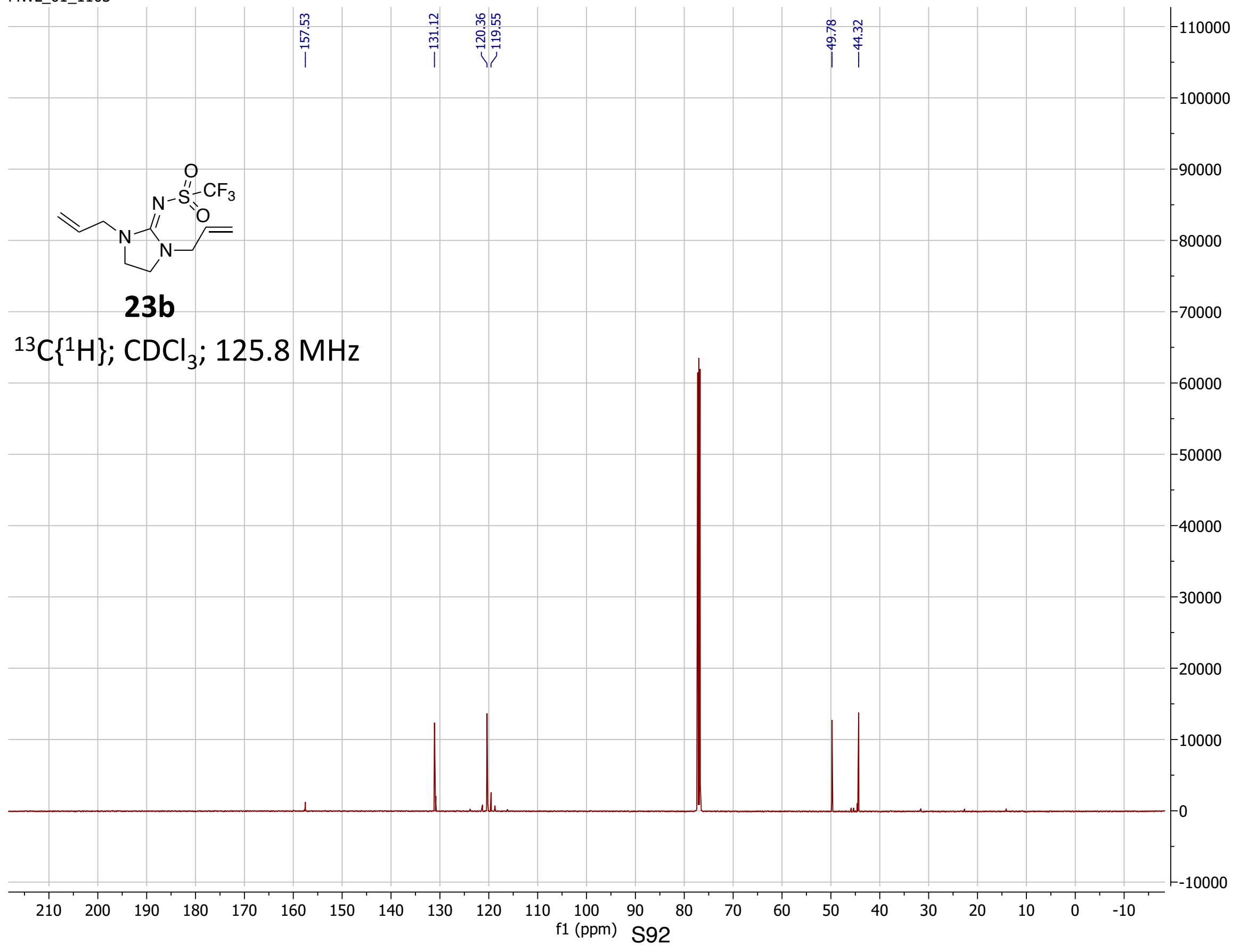




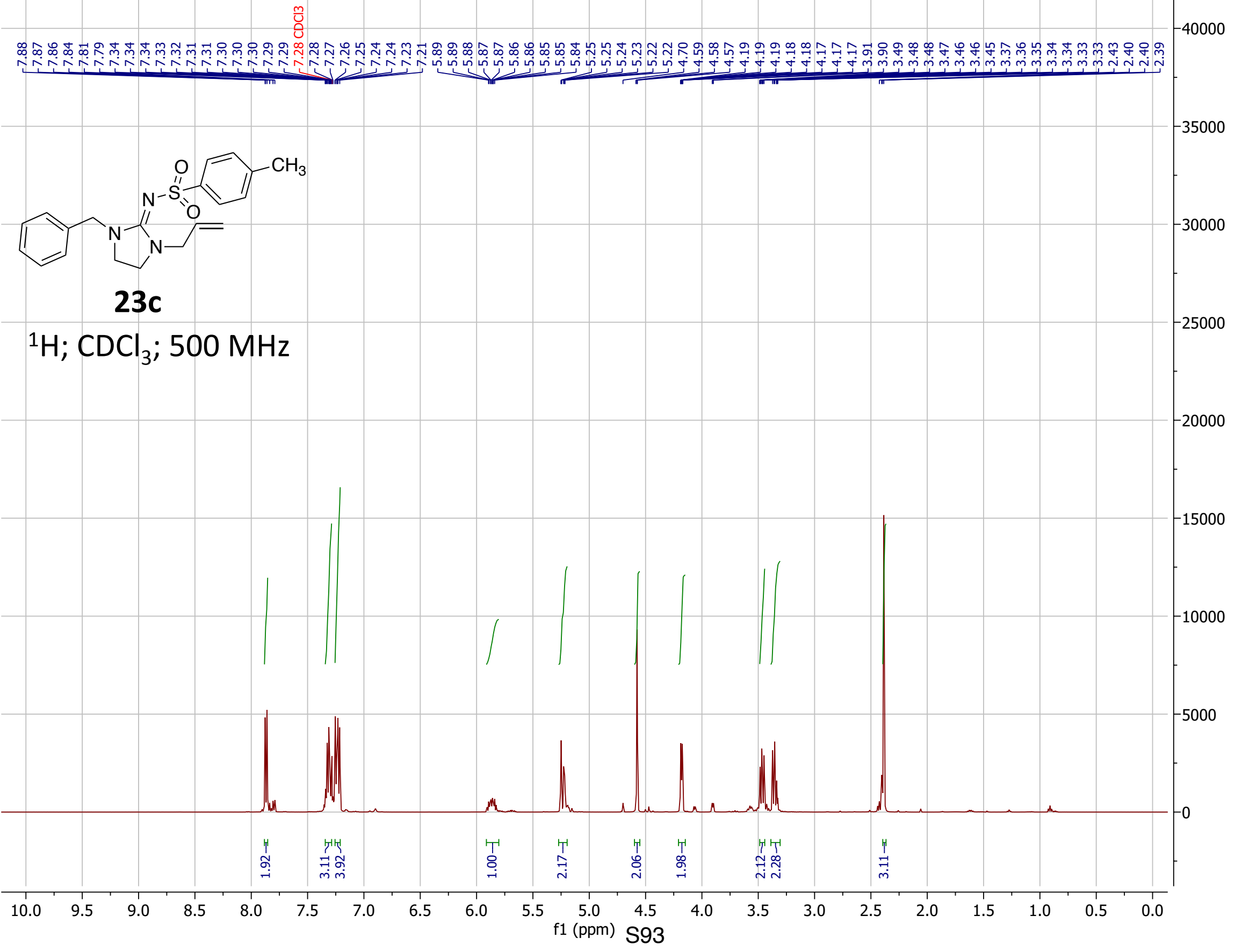




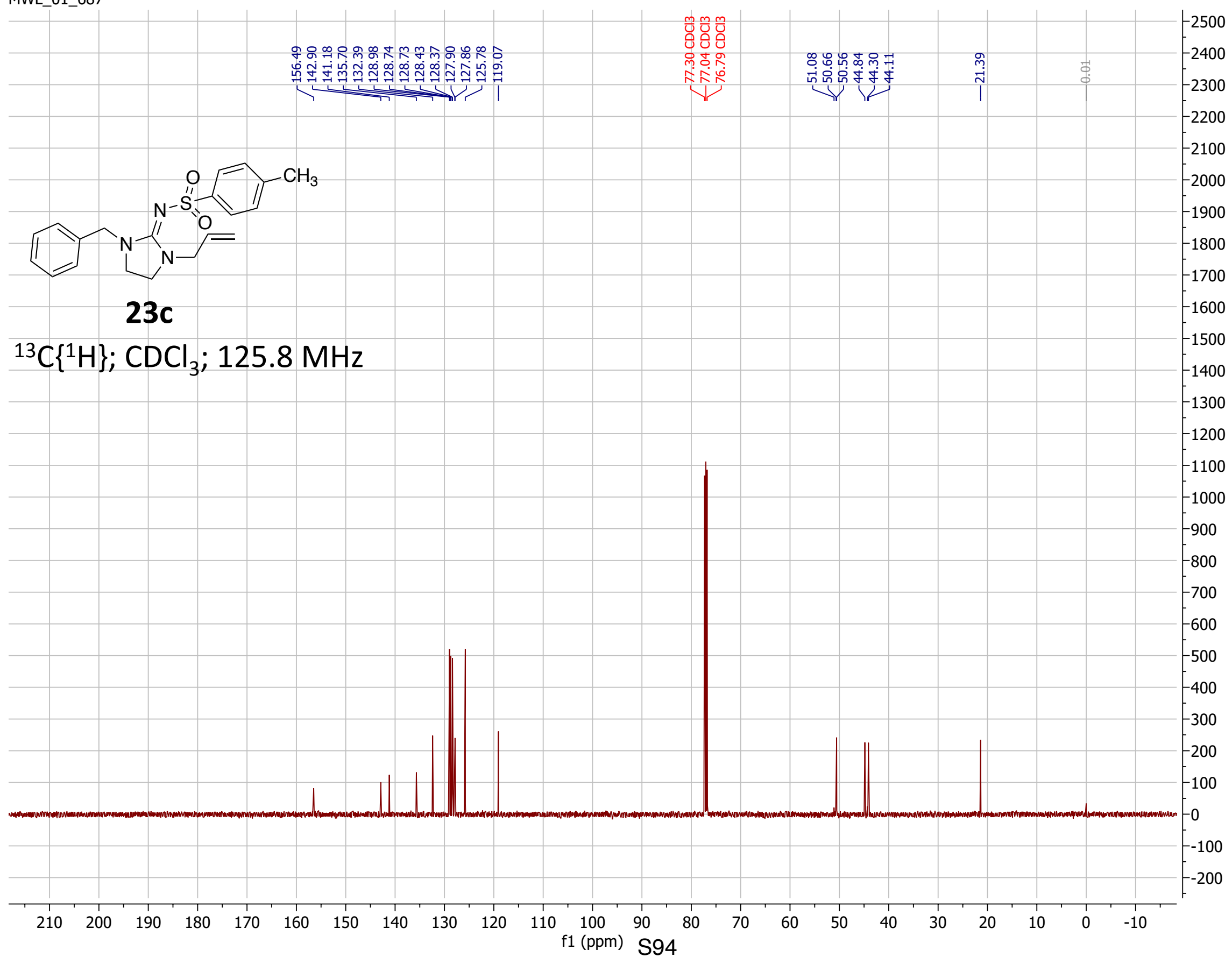


23d

\section{${ }^{1} \mathrm{H} ; \mathrm{CDCl}_{3} ; 500 \mathrm{MHz}$}

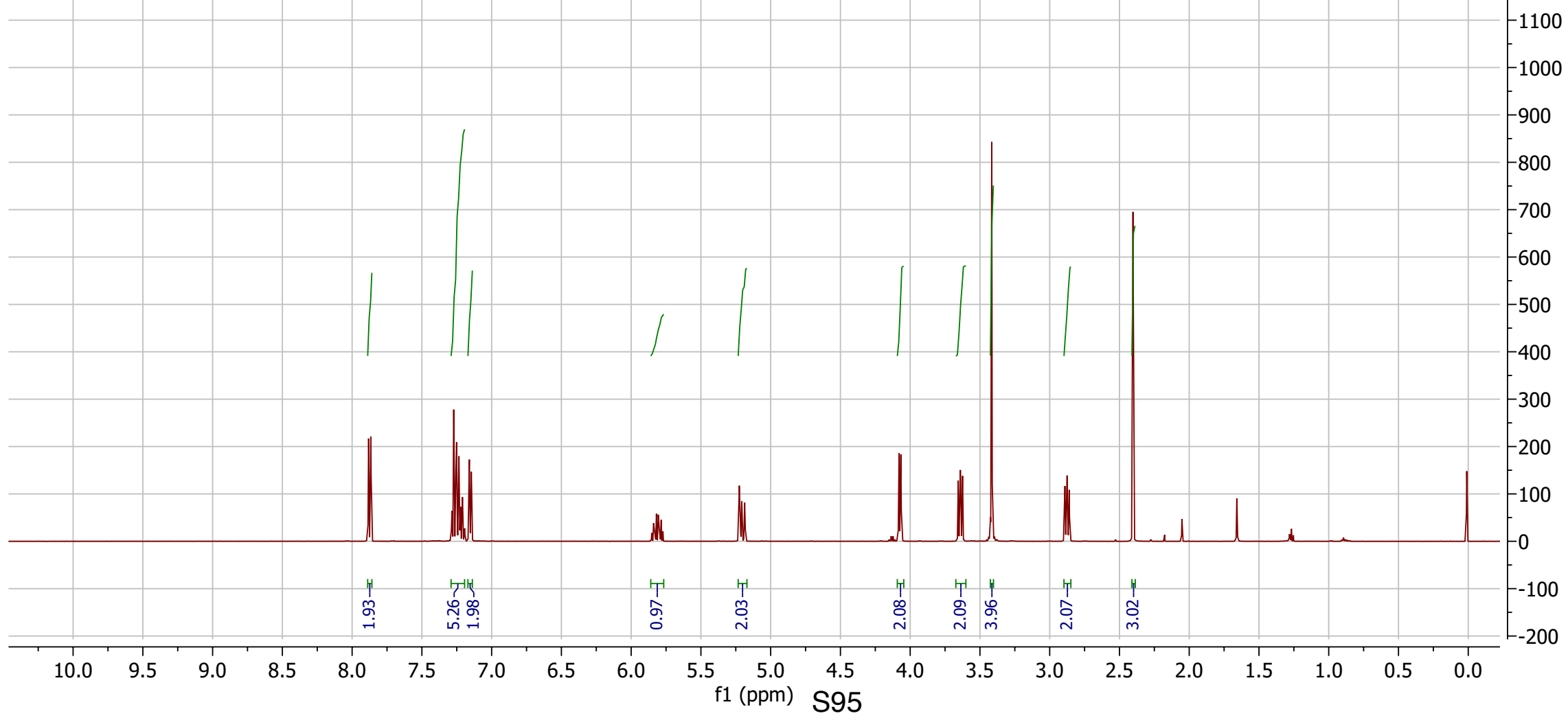




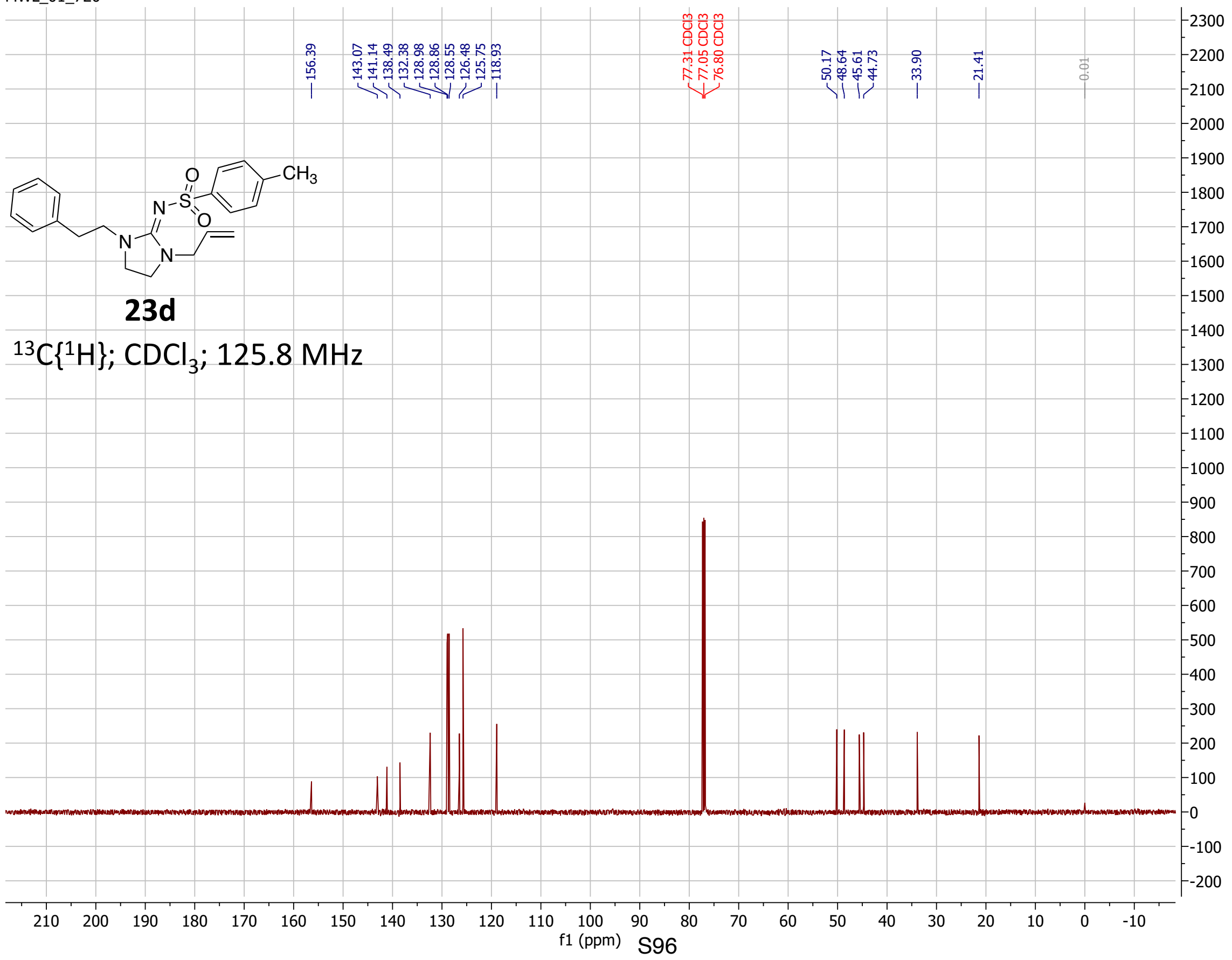




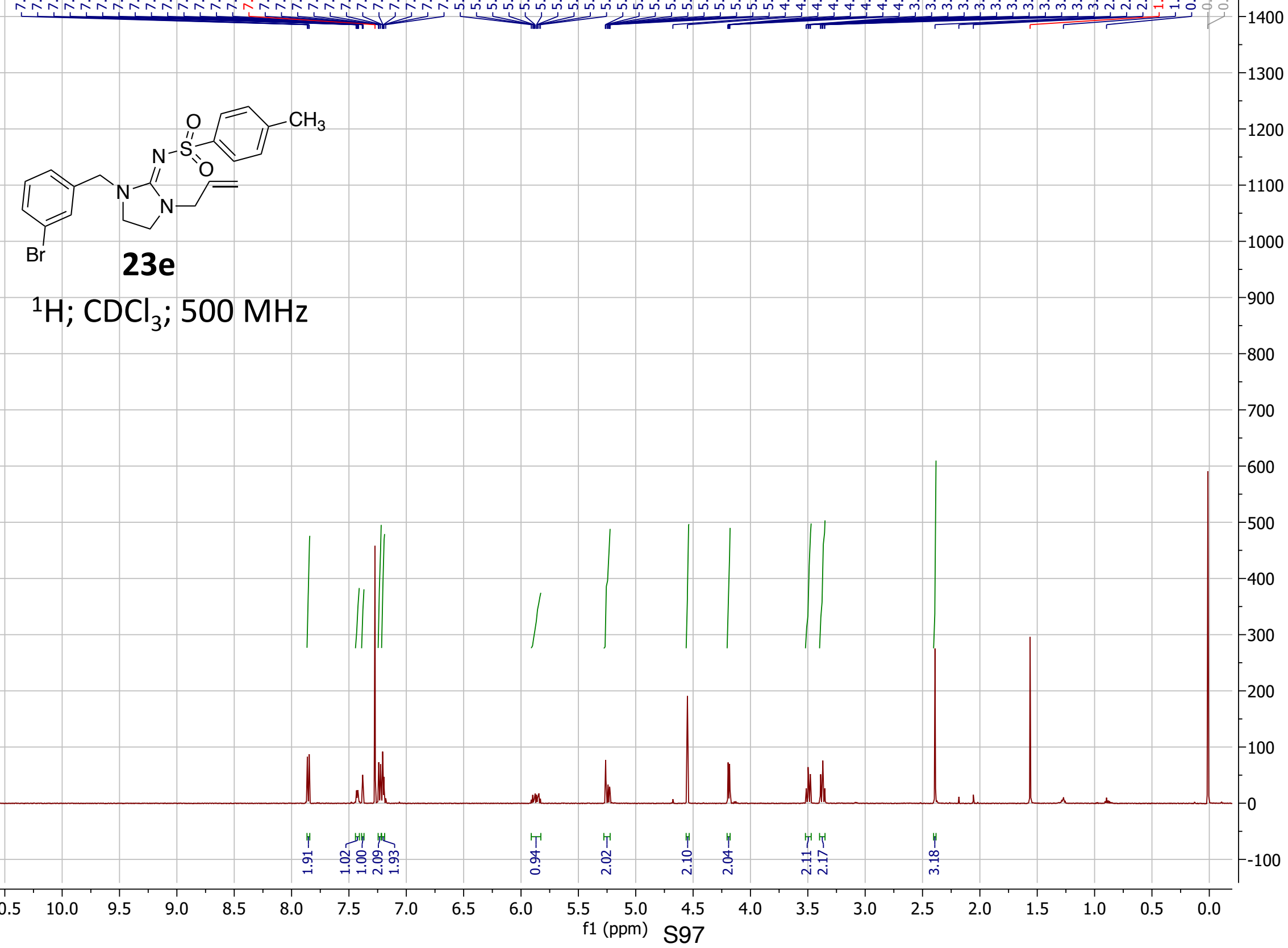




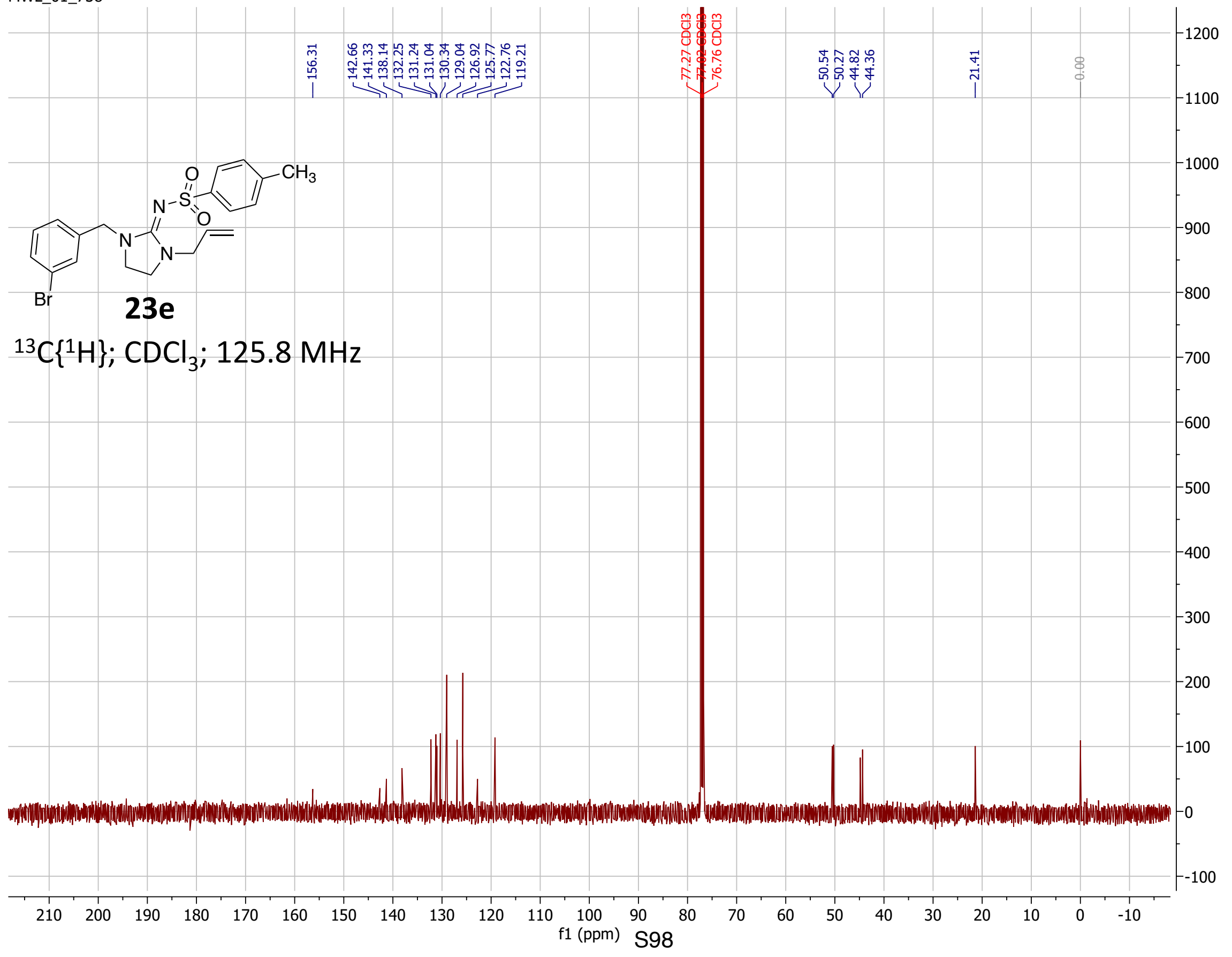




\section{$23 f$}

${ }^{1} \mathrm{H} ; \mathrm{CDCl}_{3} ; 500 \mathrm{MHz}$

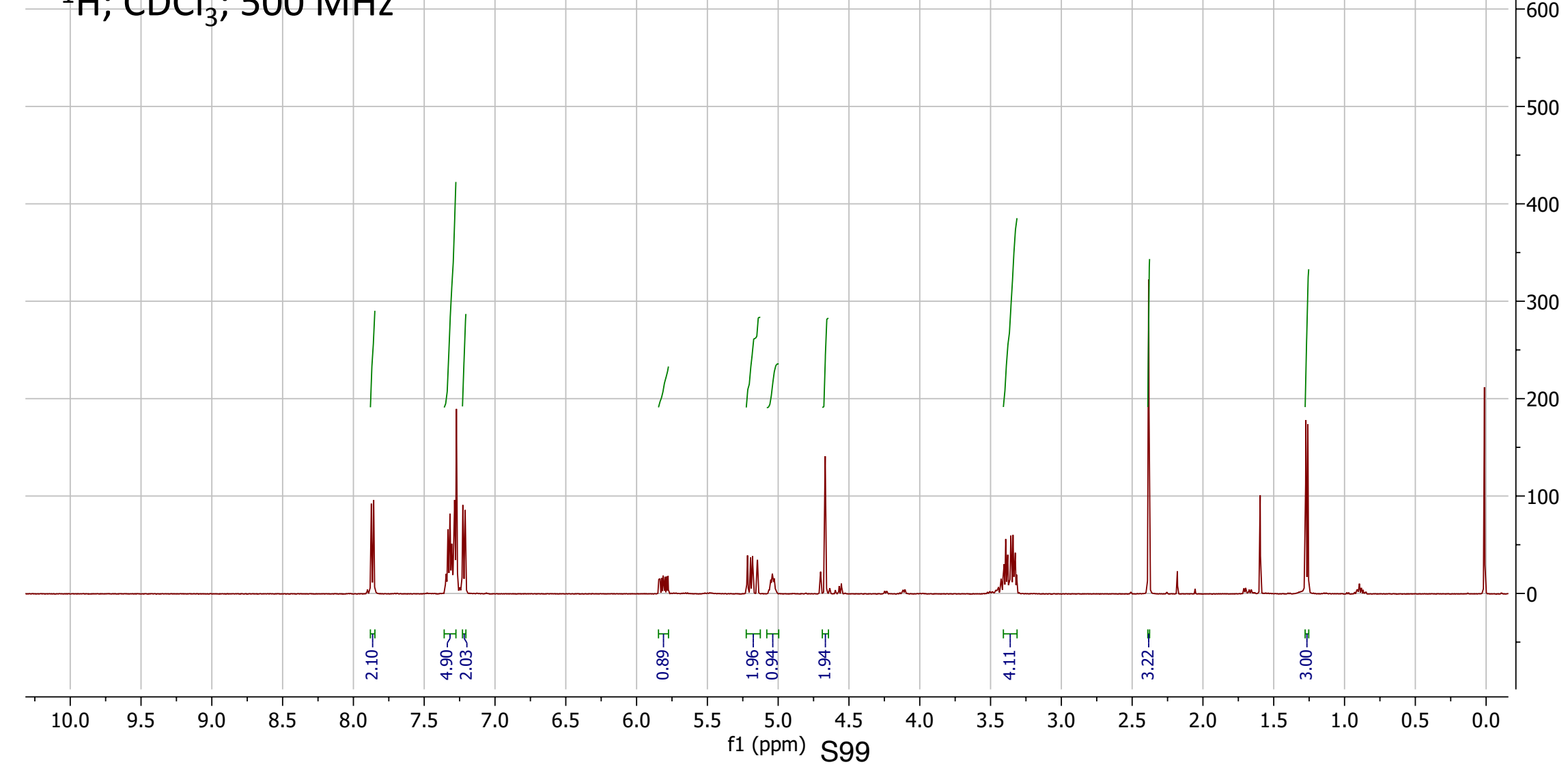




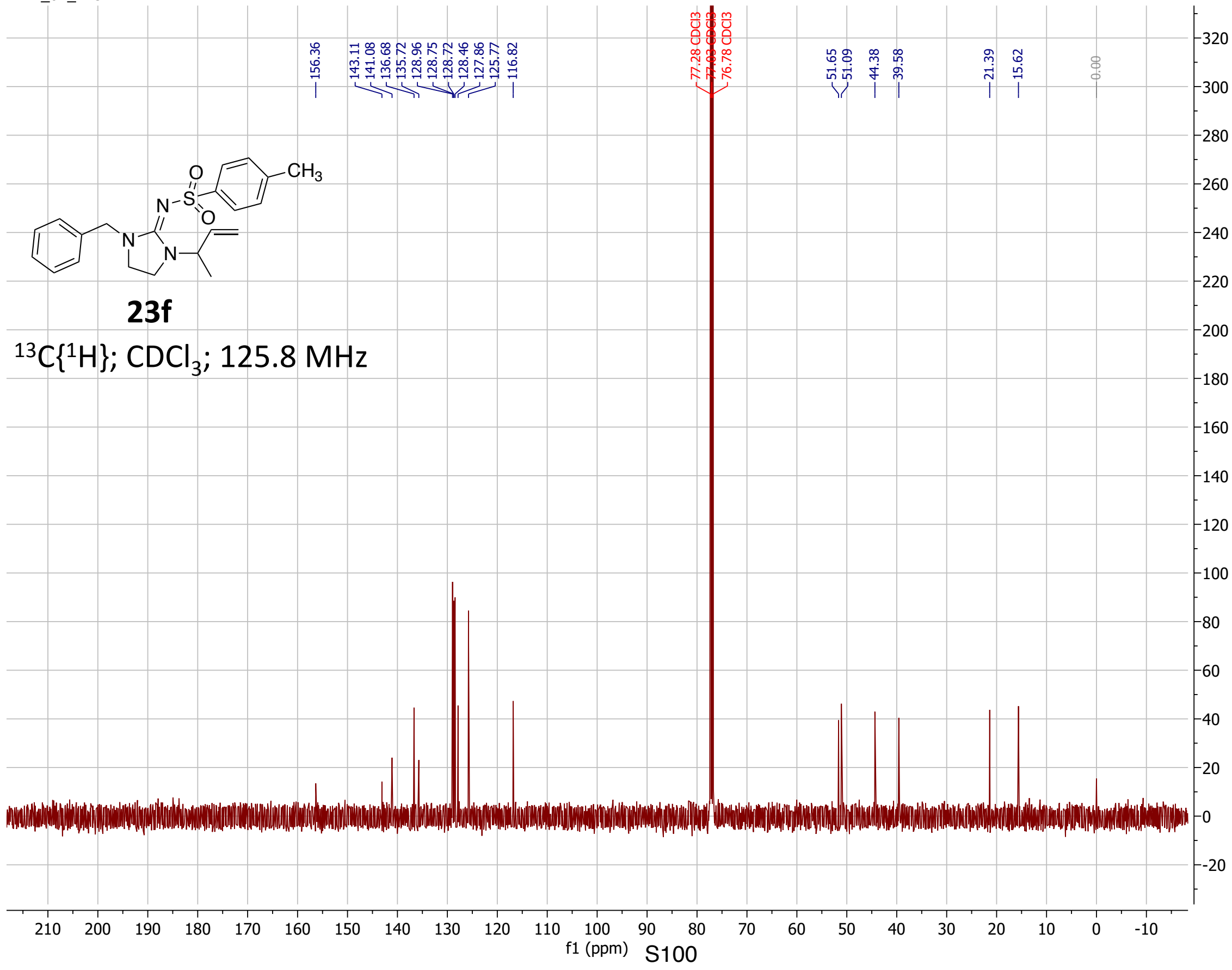




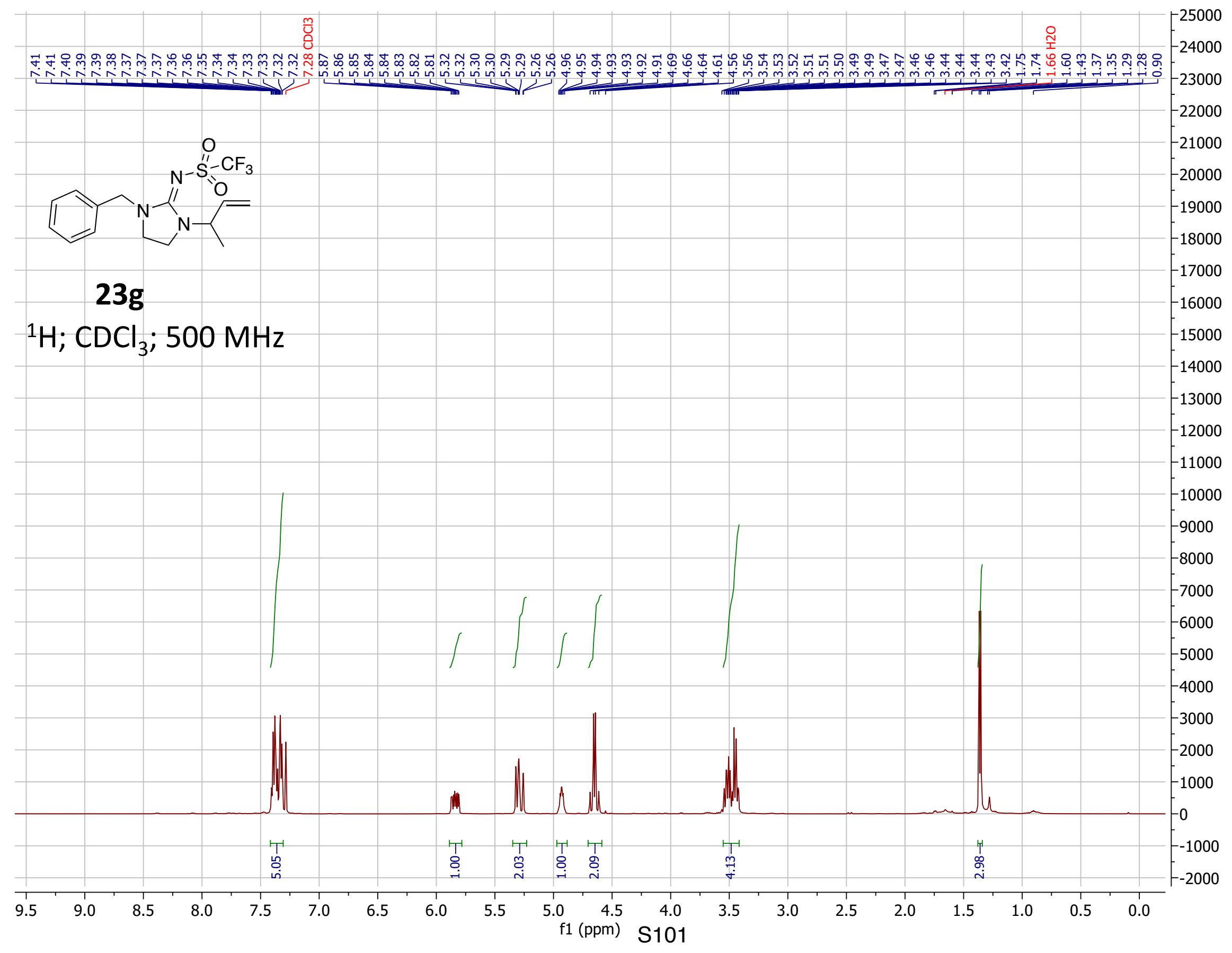




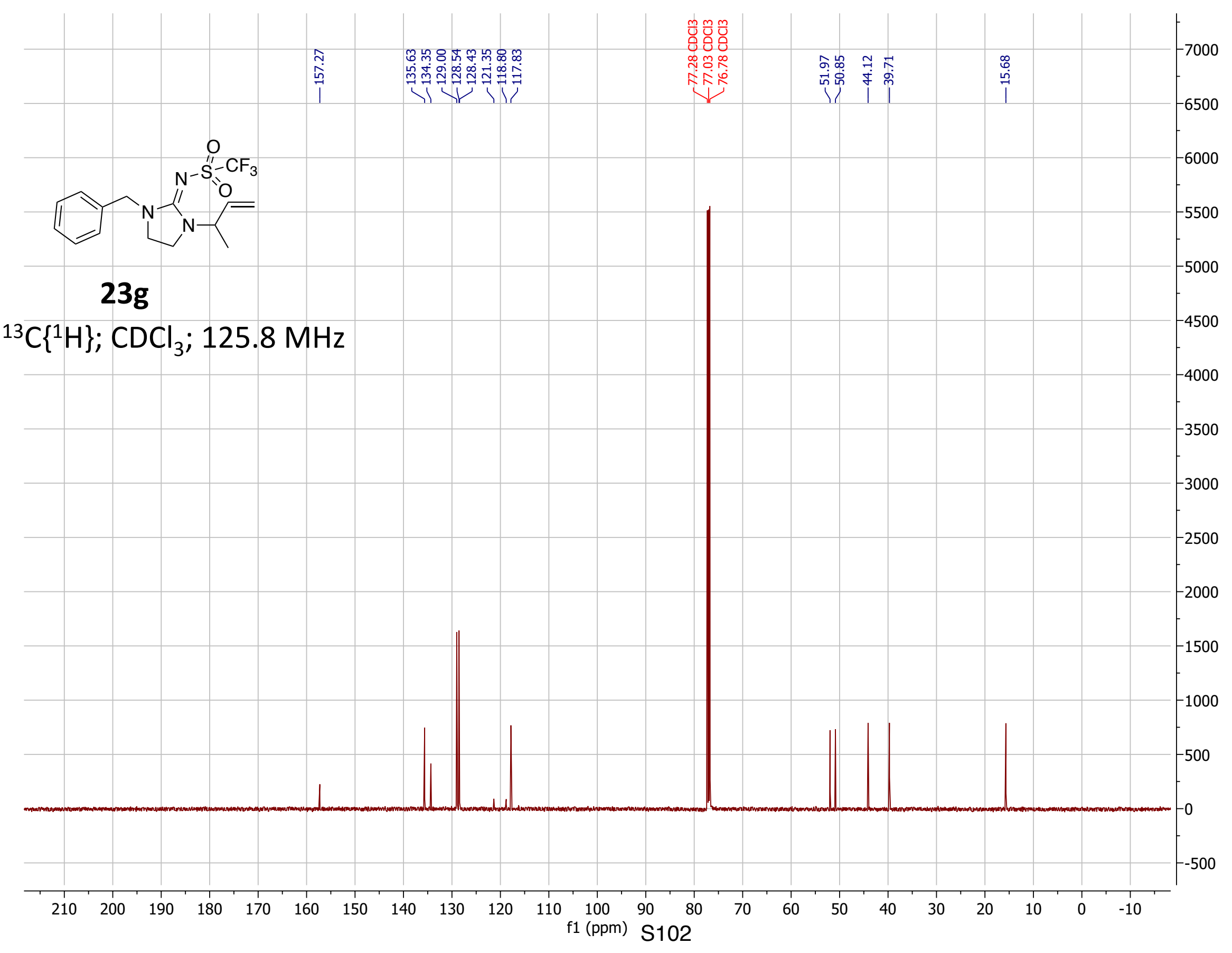




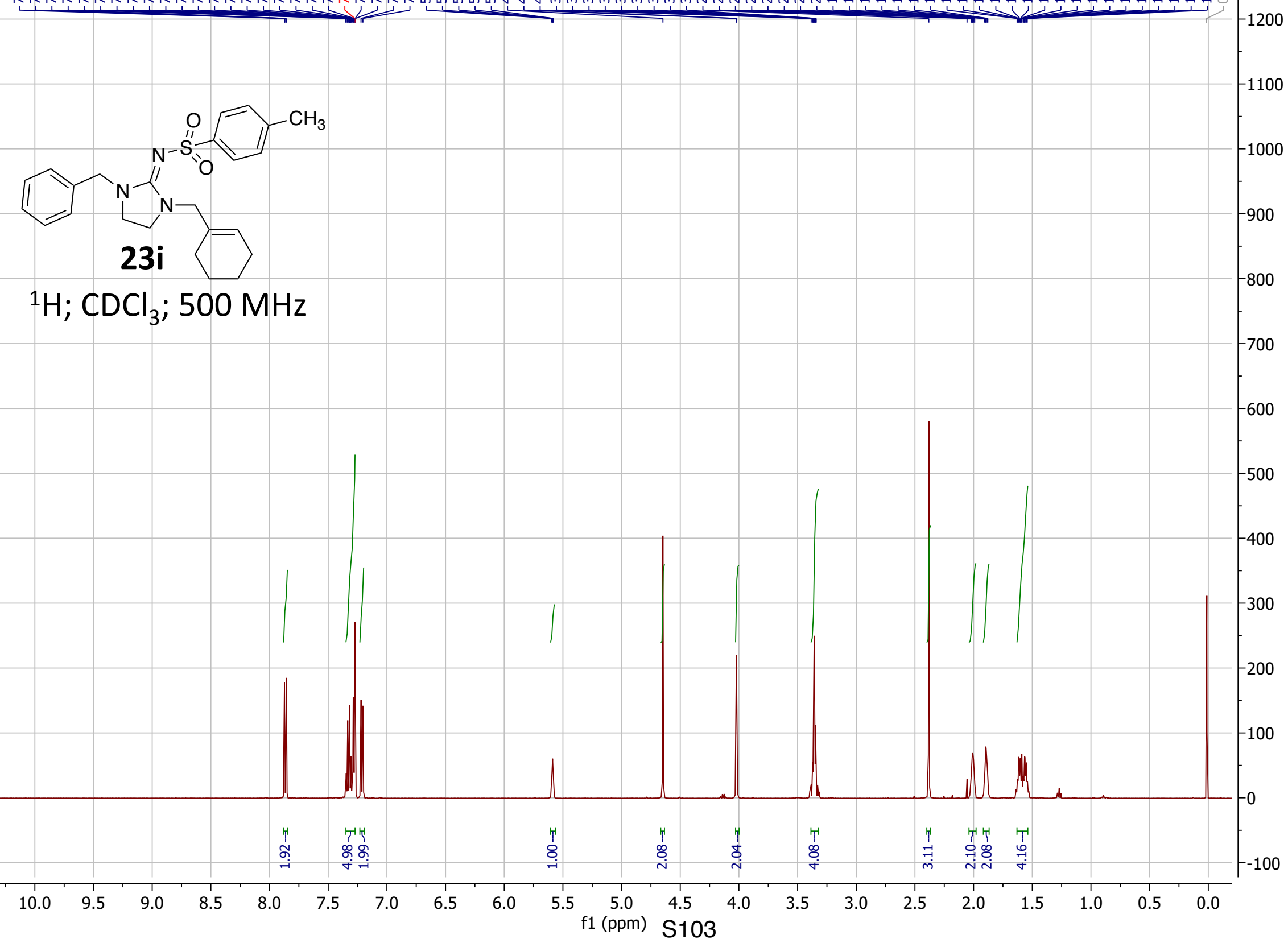




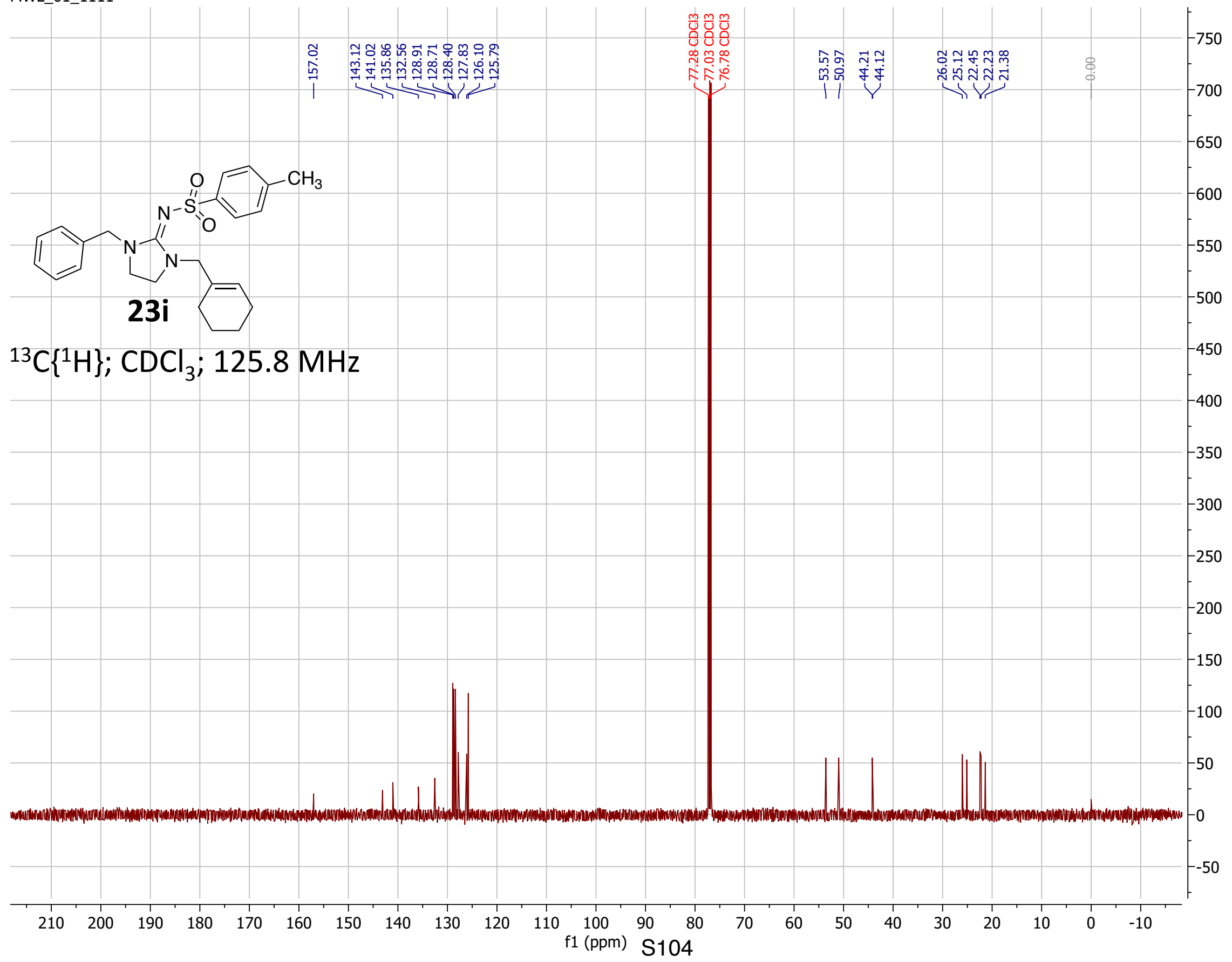


(-)

25

${ }^{1} \mathrm{H} ; \mathrm{CDCl}_{3} ; 500 \mathrm{MHz}$
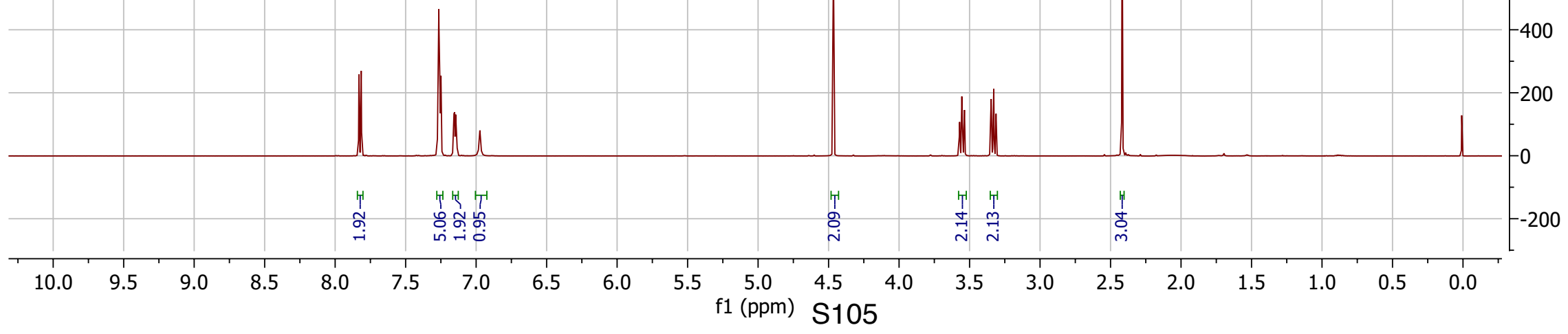


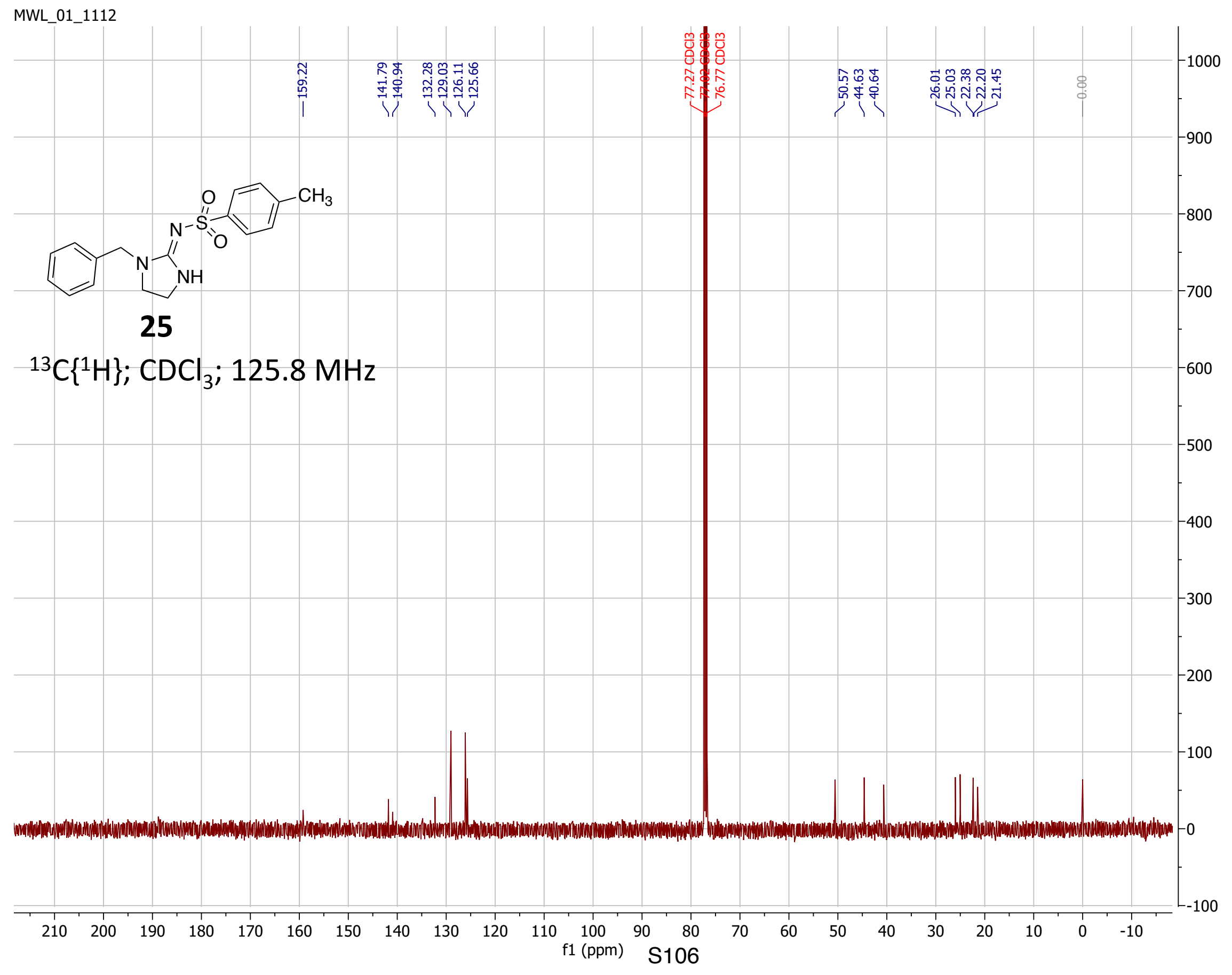




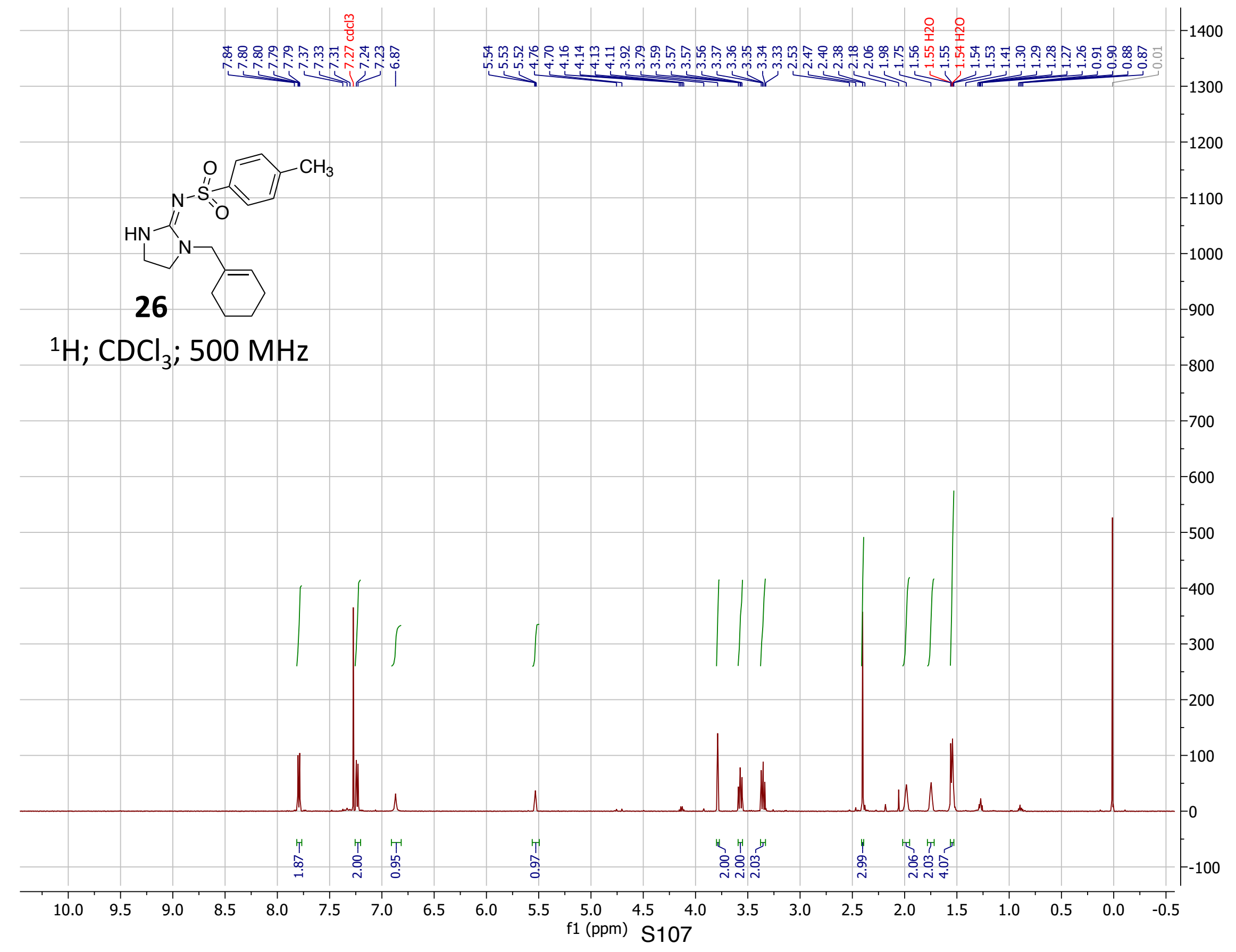




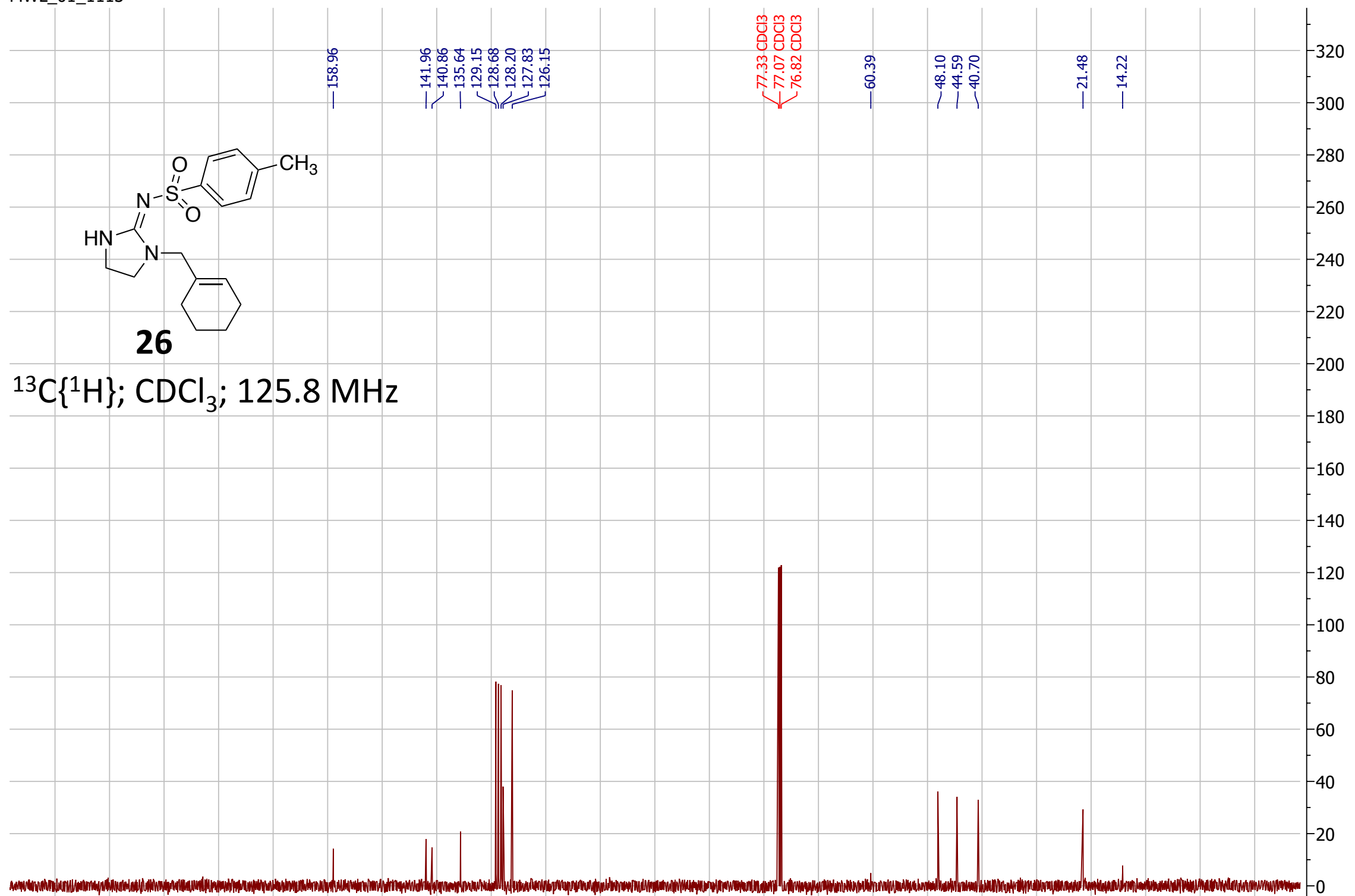




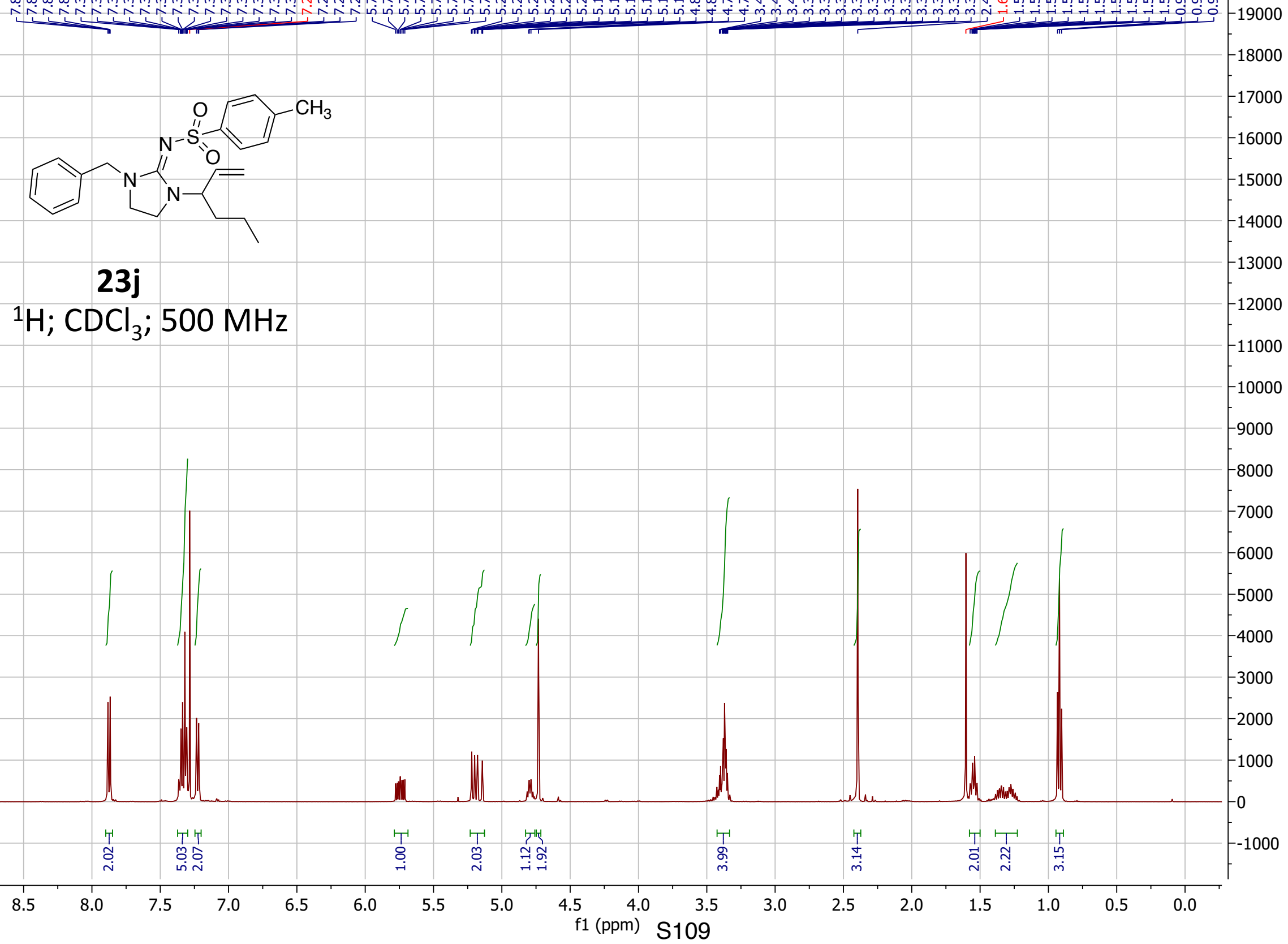




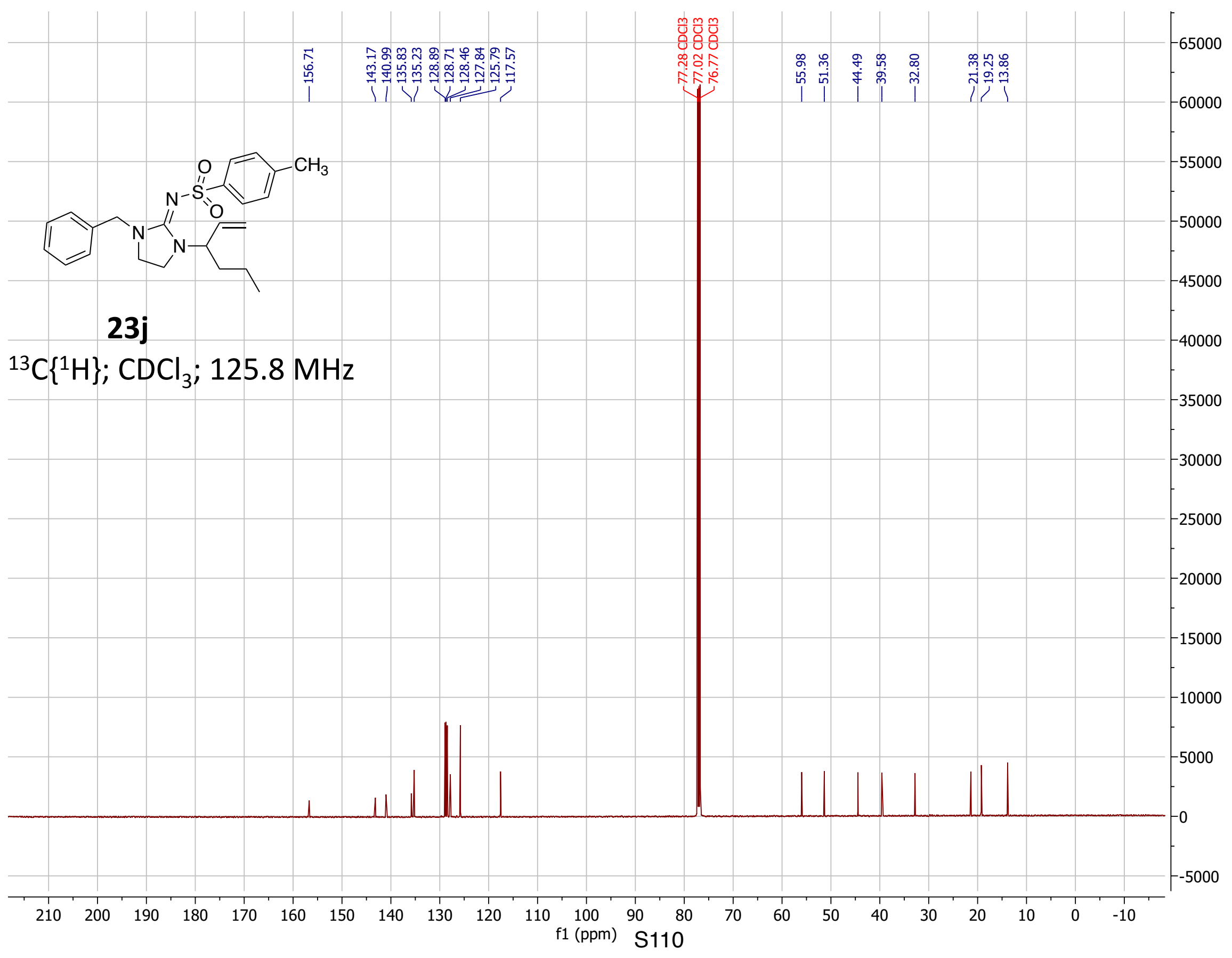




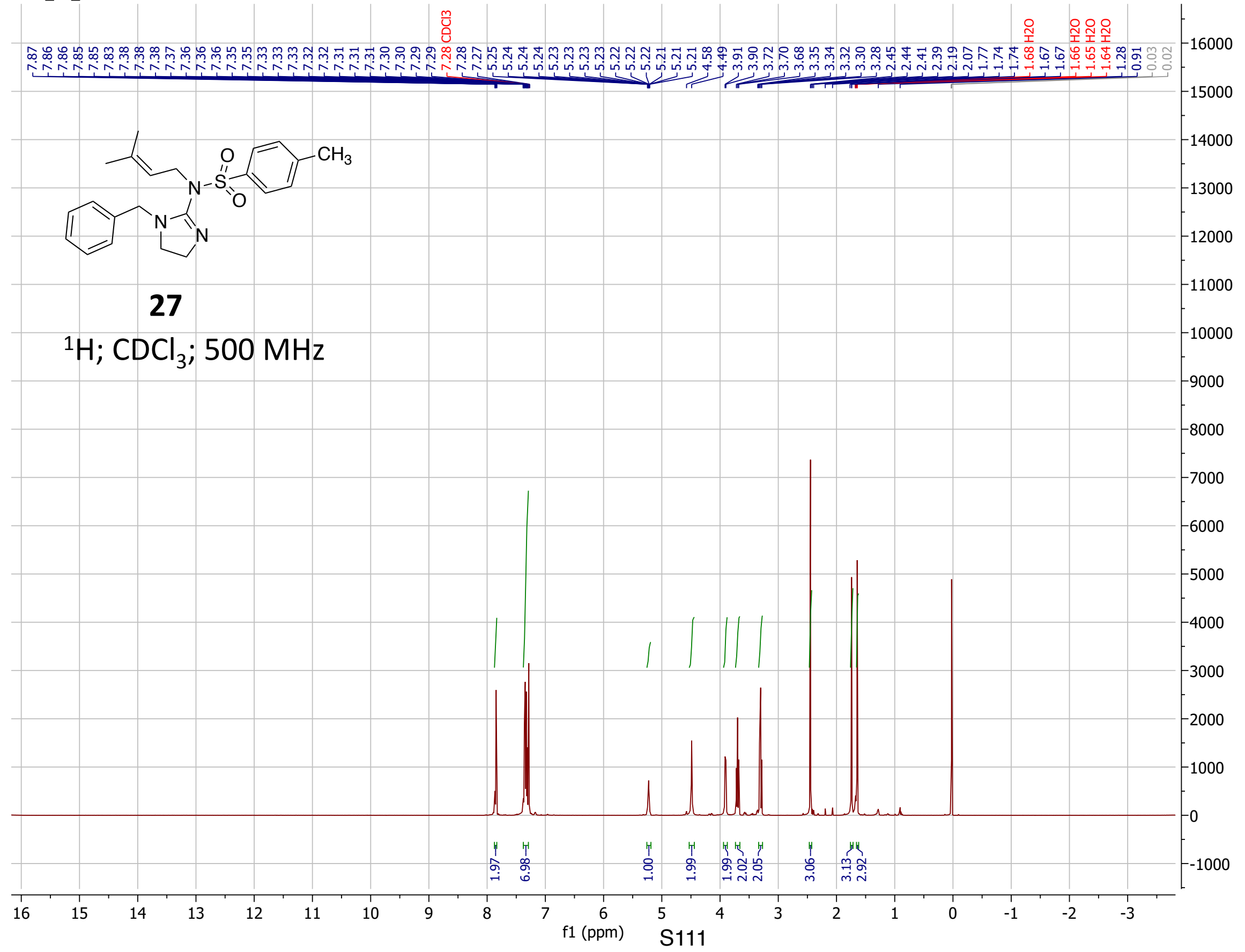




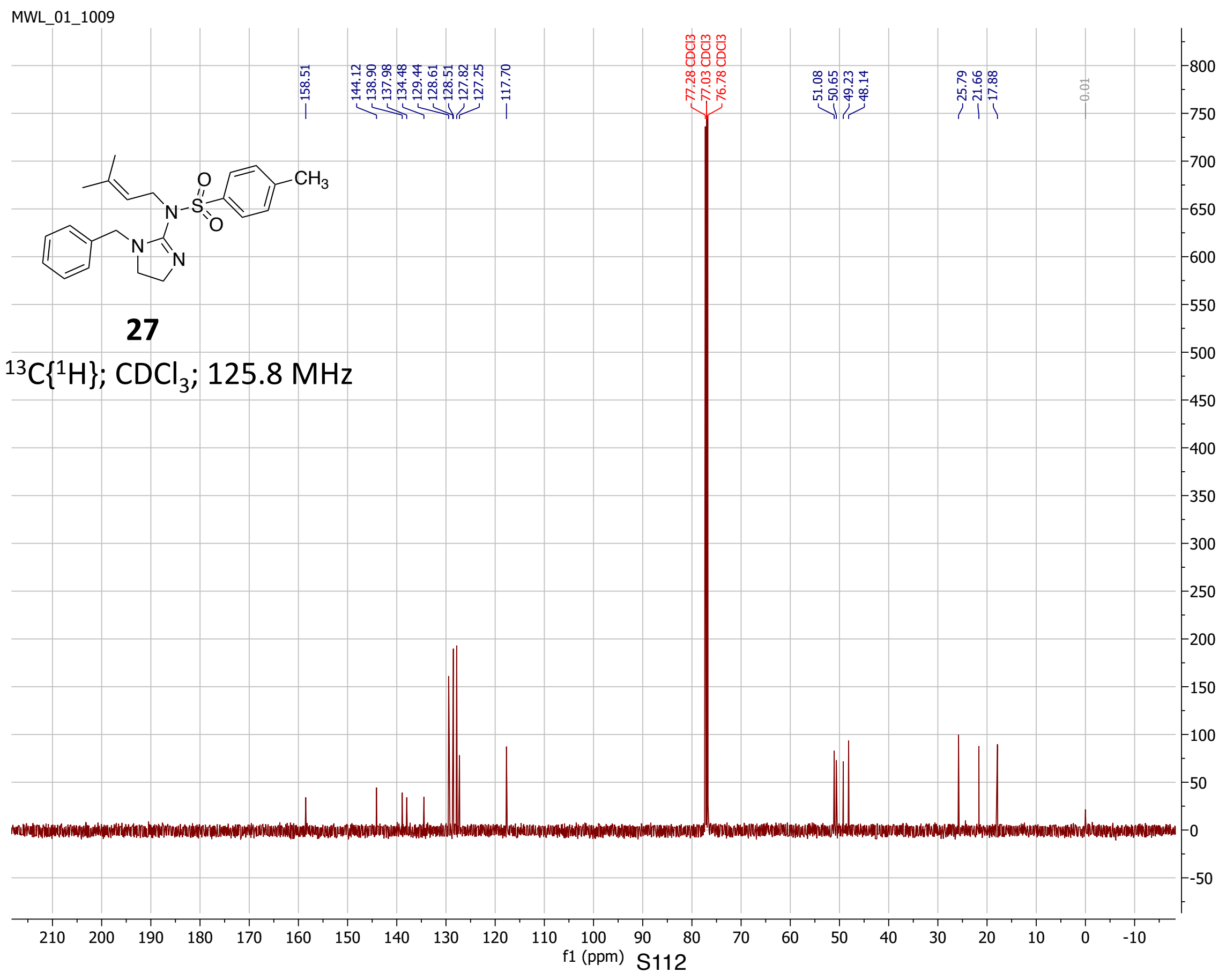




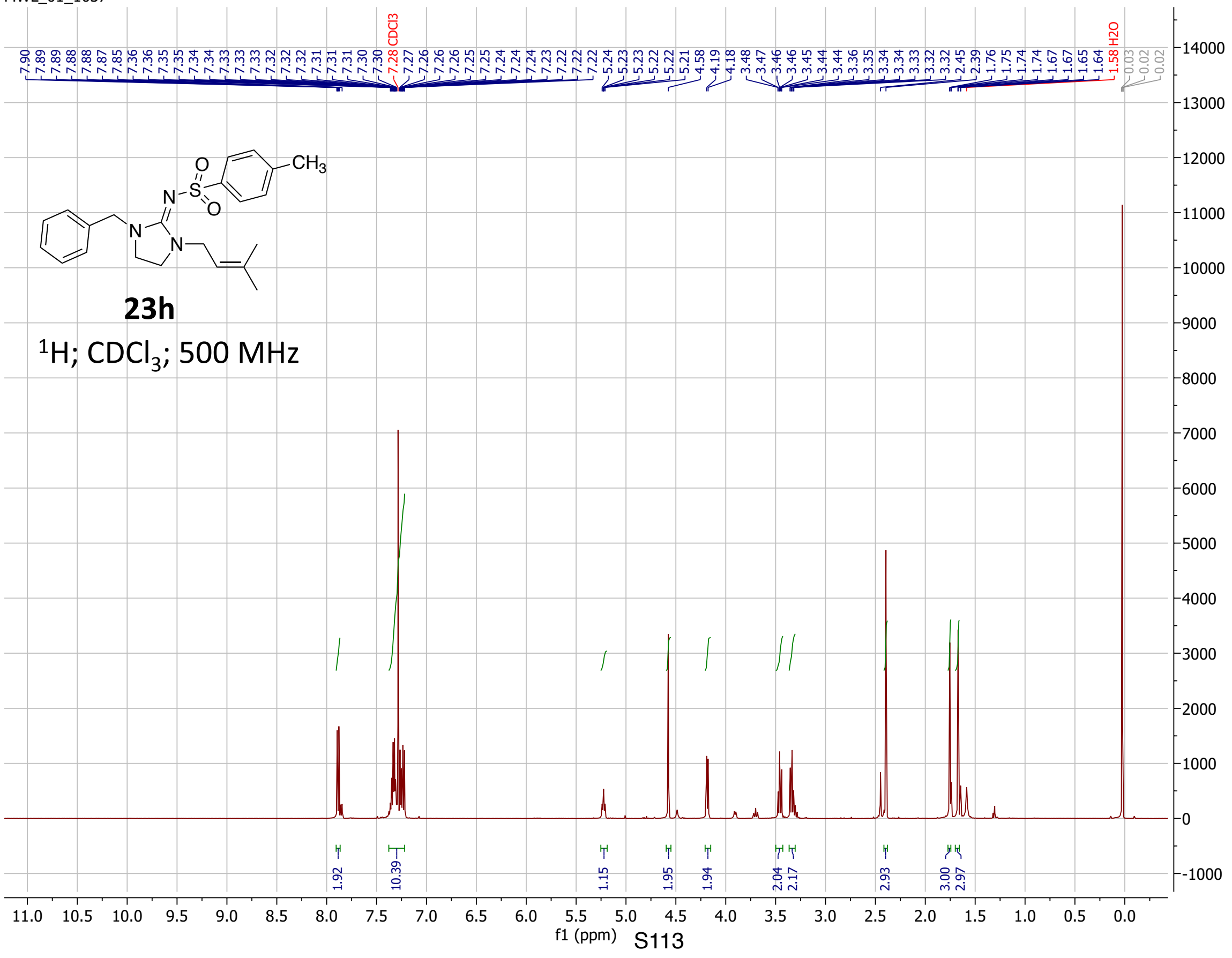



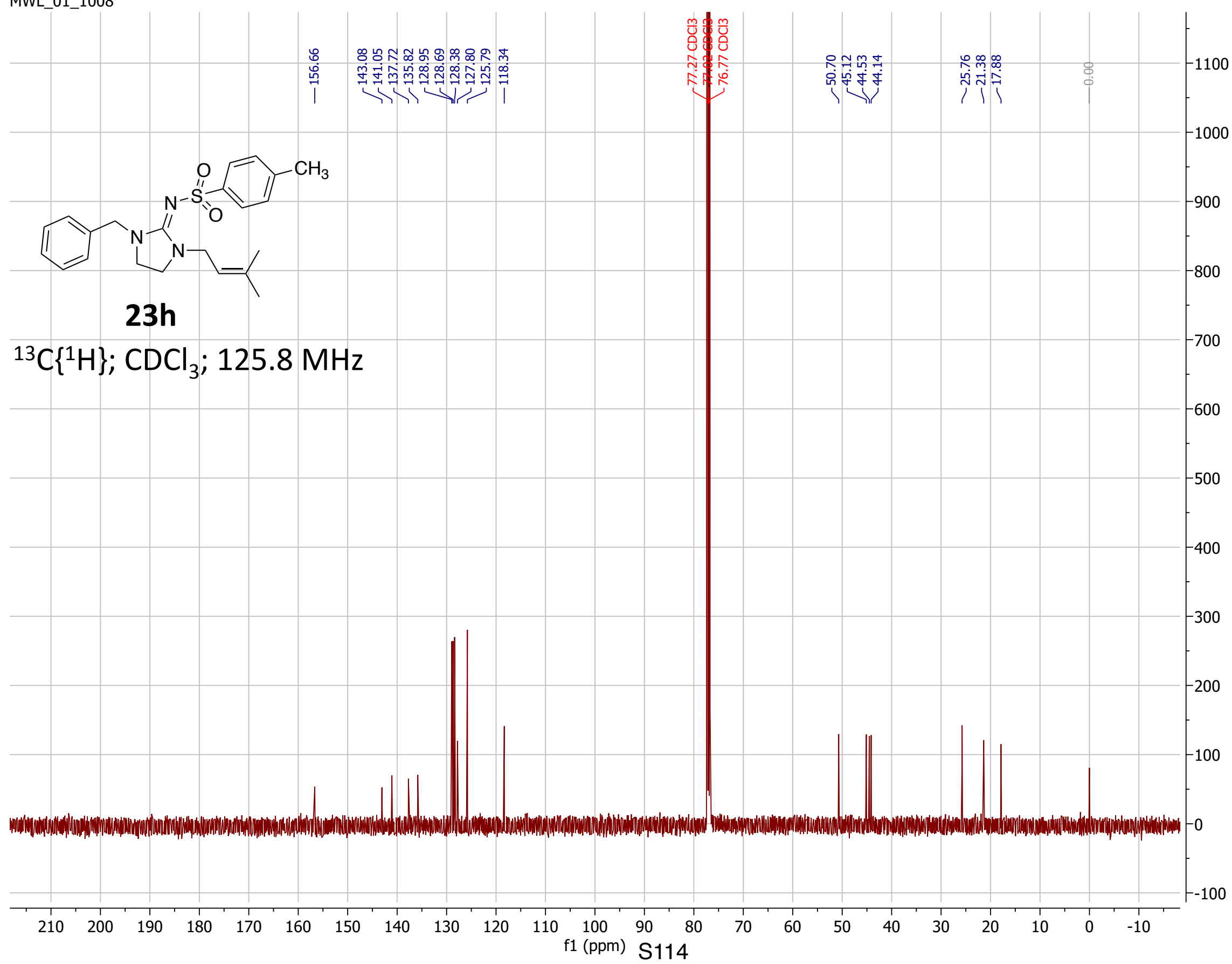


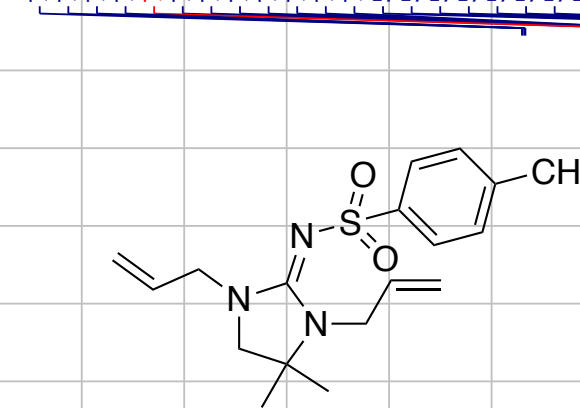

28

\section{${ }^{1} \mathrm{H} ; \mathrm{CDCl}_{3} ; 500 \mathrm{MHz}$}

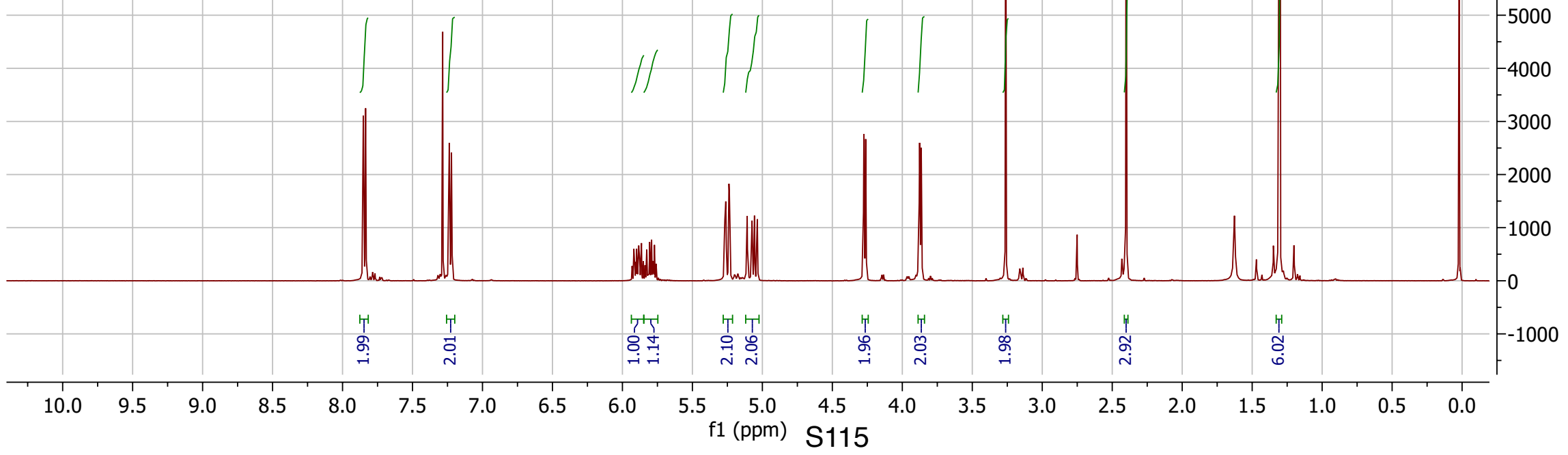




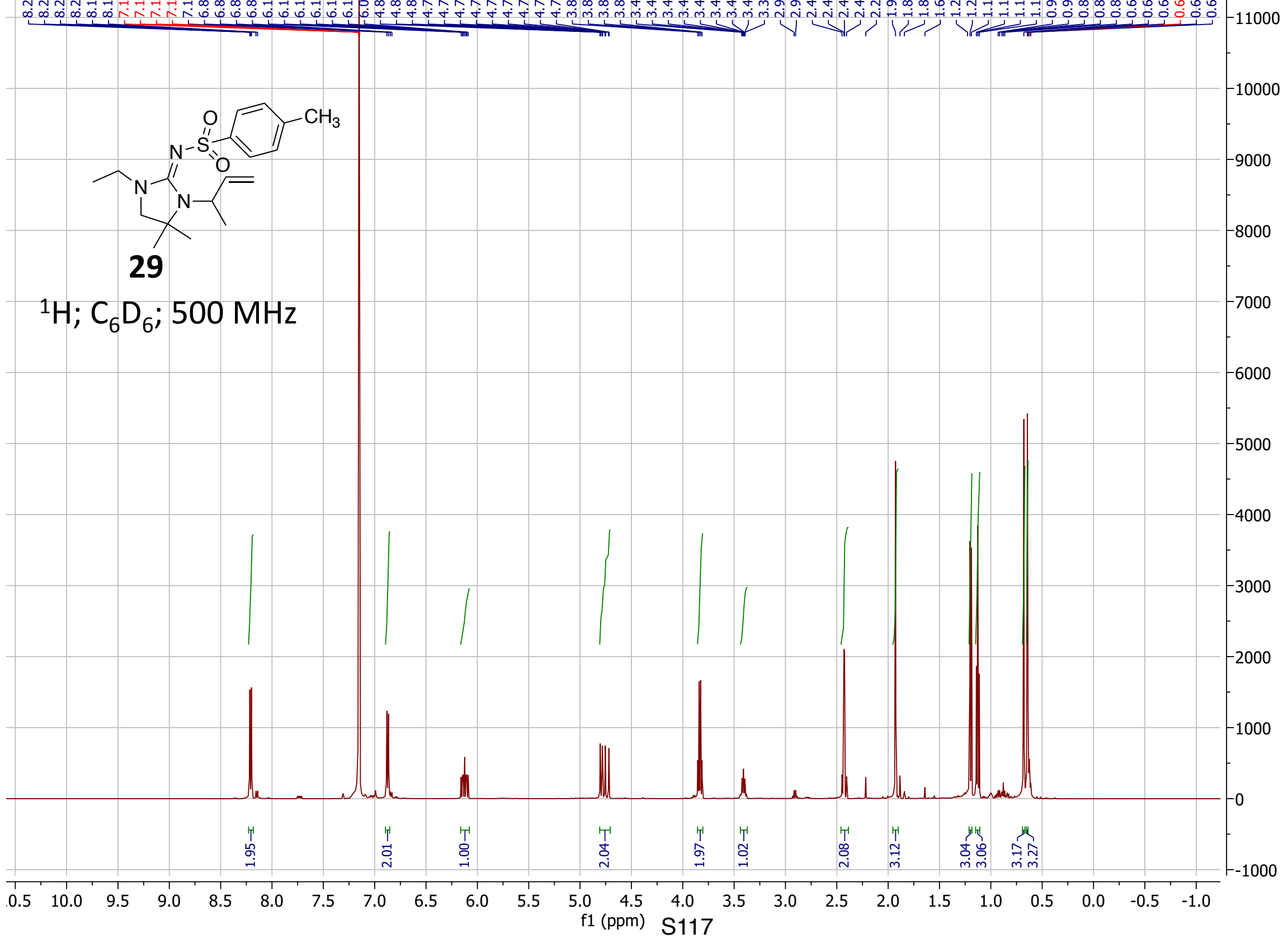




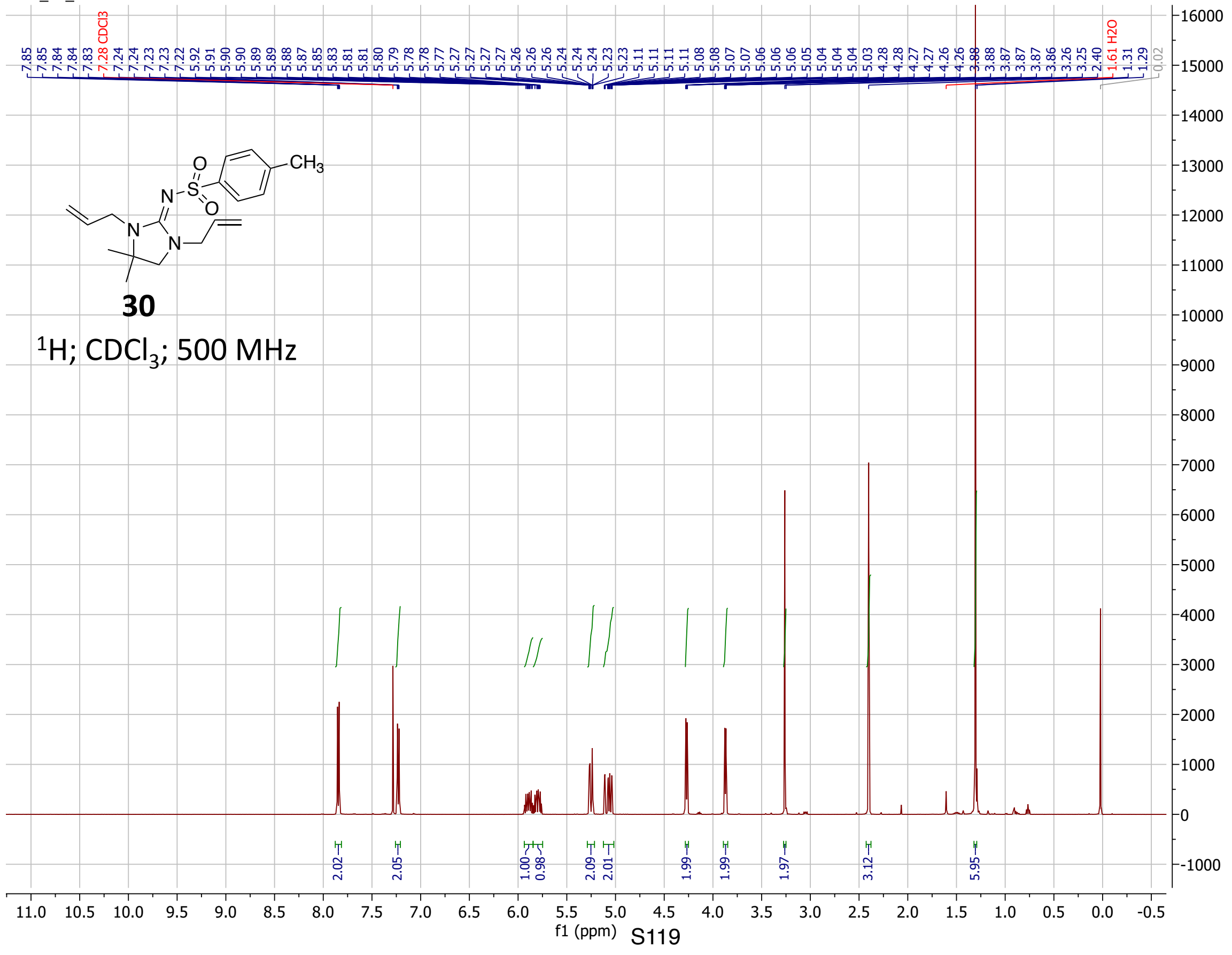




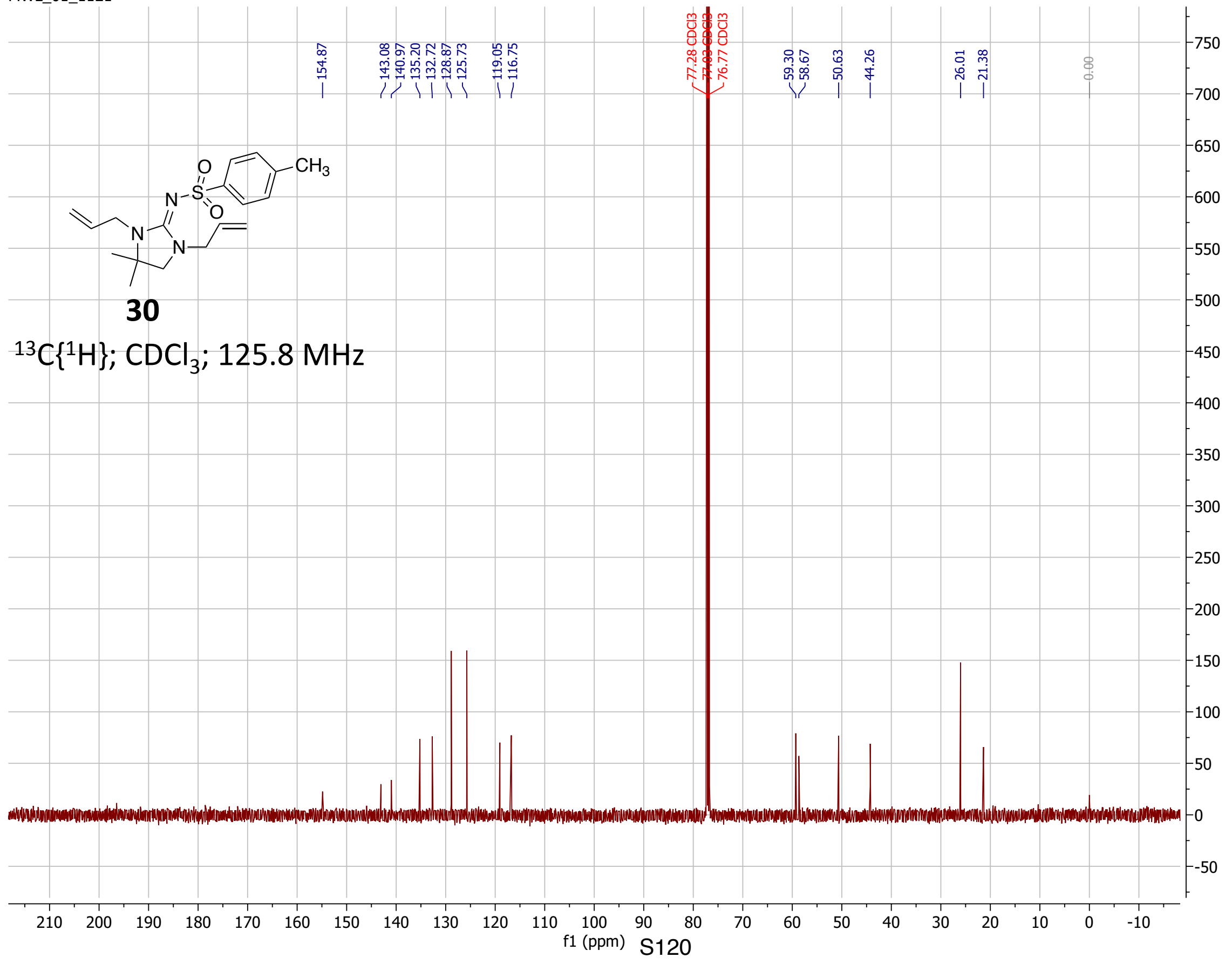




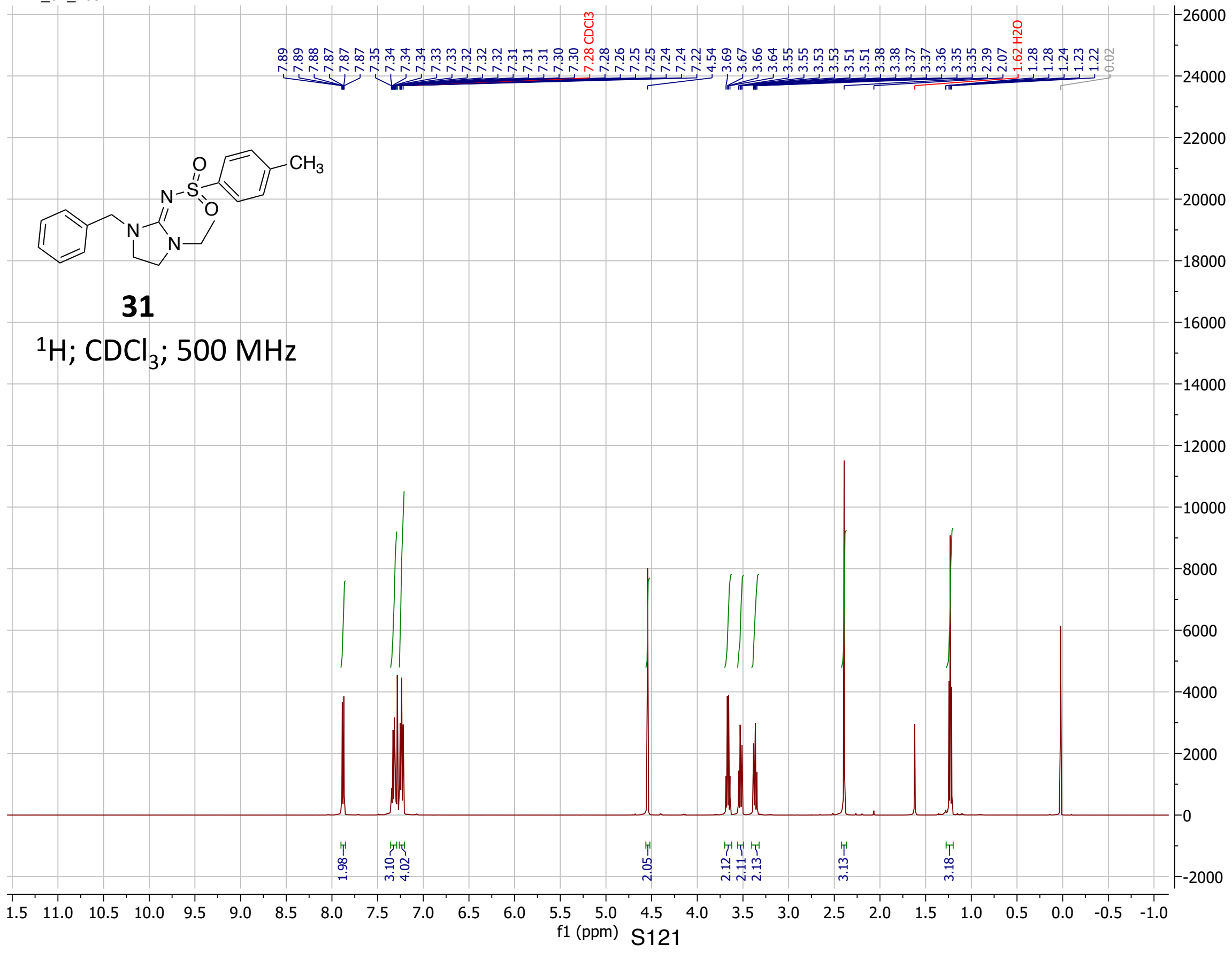




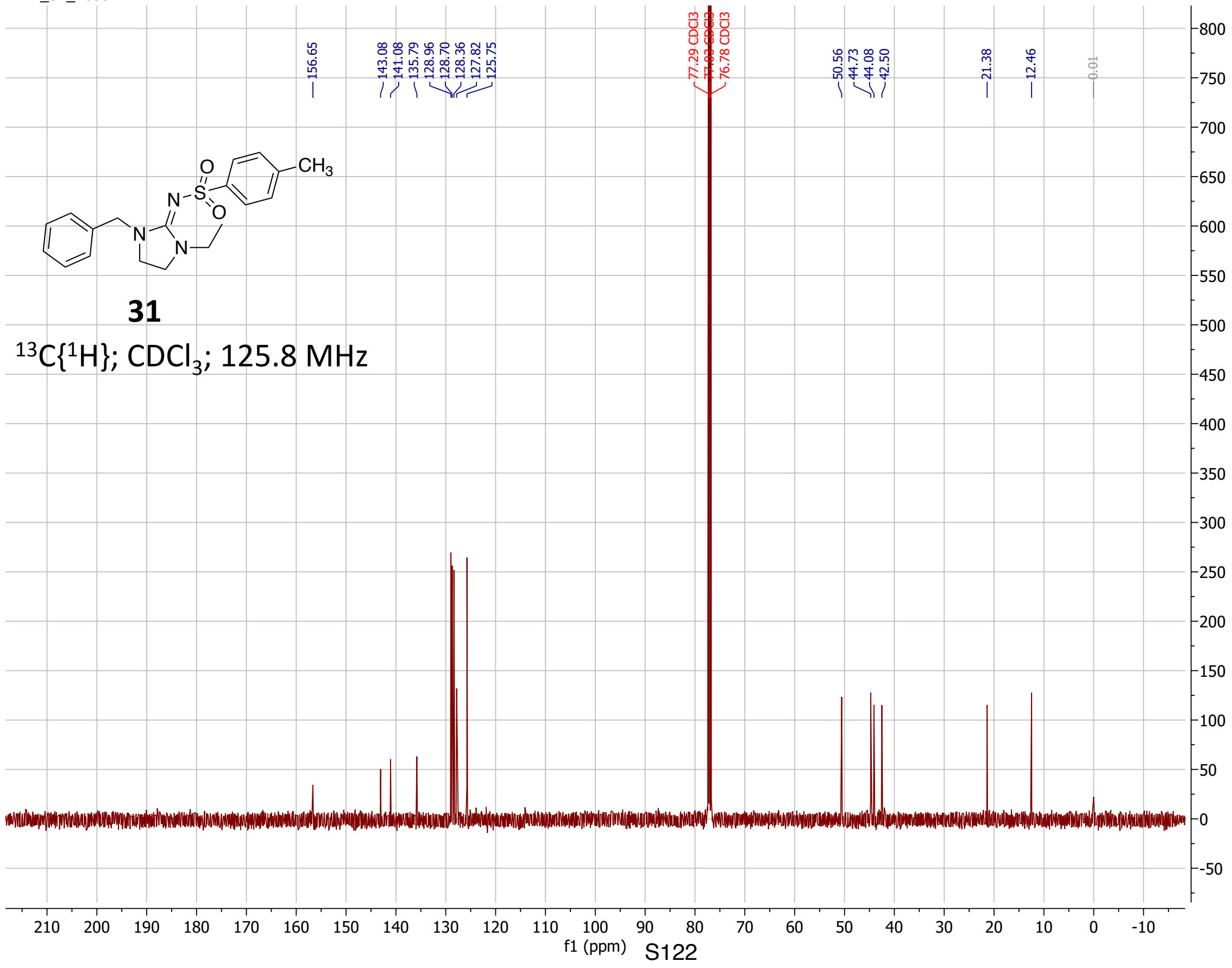




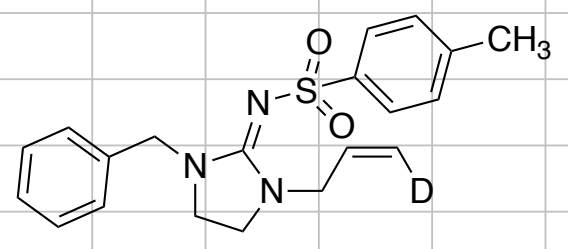

35 and 36

\section{${ }^{1} \mathrm{H} ; \mathrm{CDCl}_{3} ; 500 \mathrm{MHz}$}

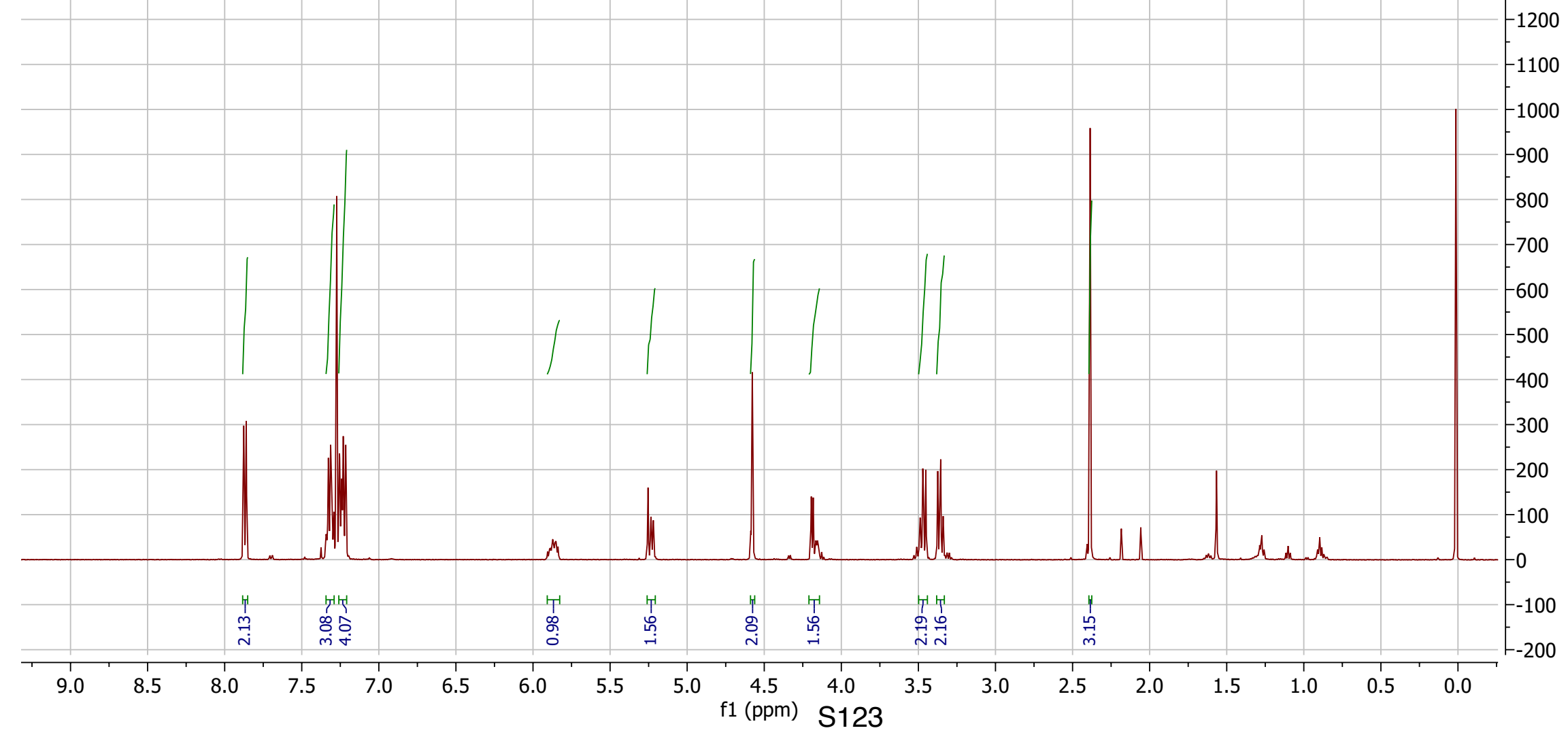




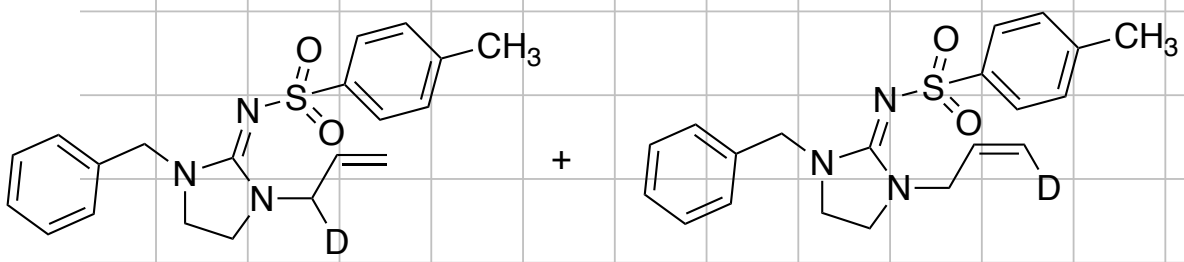

35 and 36

${ }^{13} \mathrm{C}\left\{{ }^{1} \mathrm{H}\right\} ; \mathrm{CDCl}_{3} ; 125.8 \mathrm{MHz}$

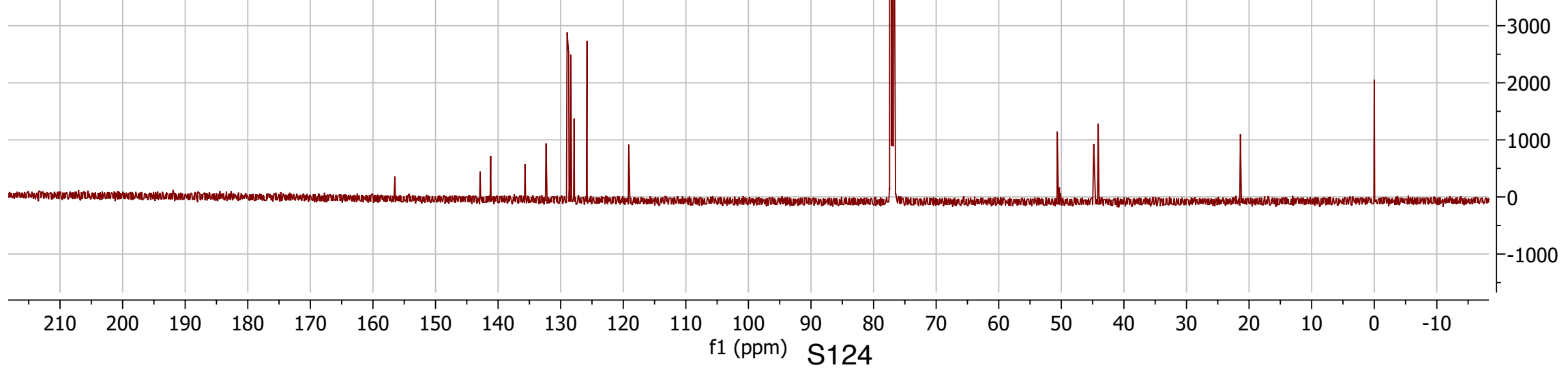




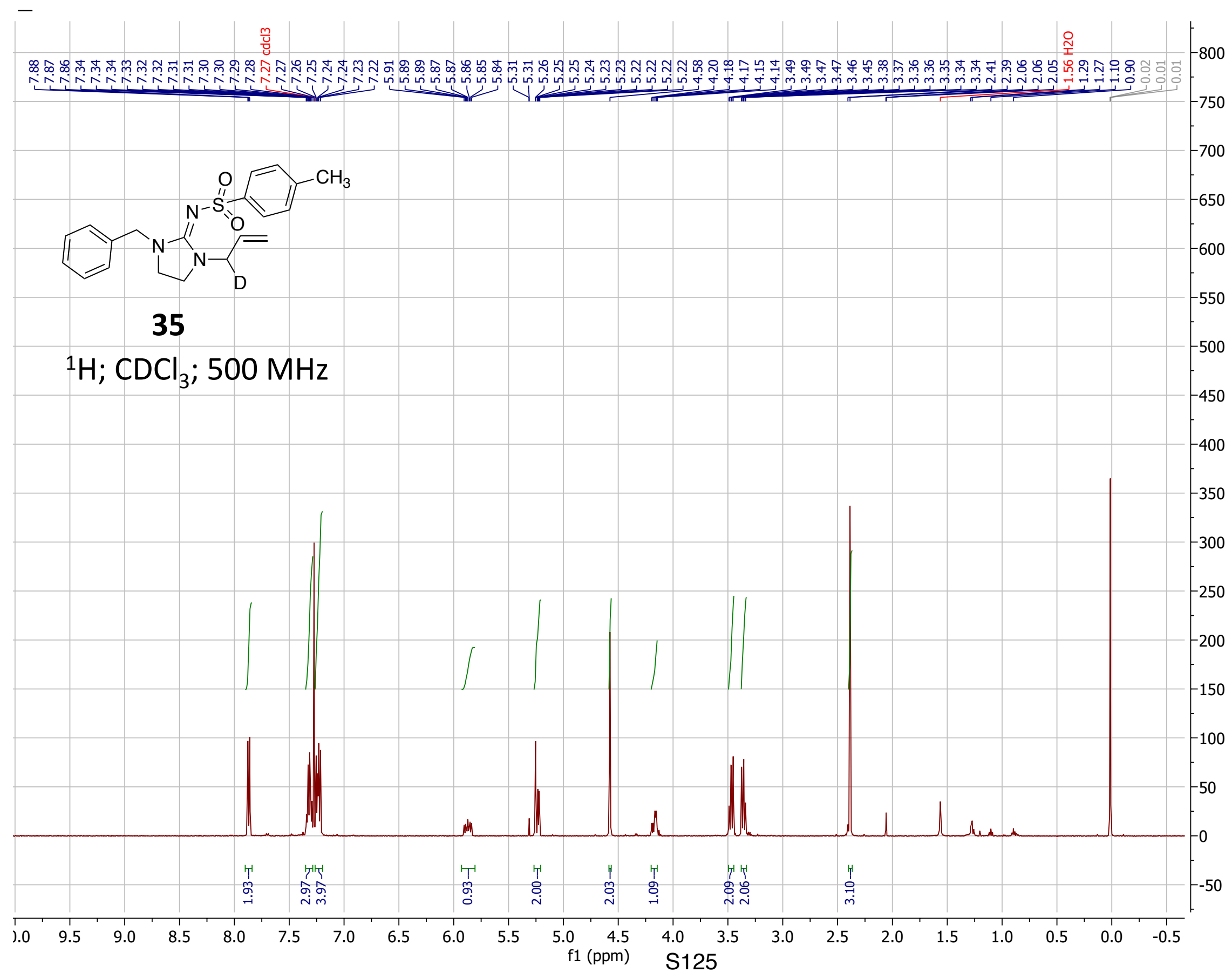




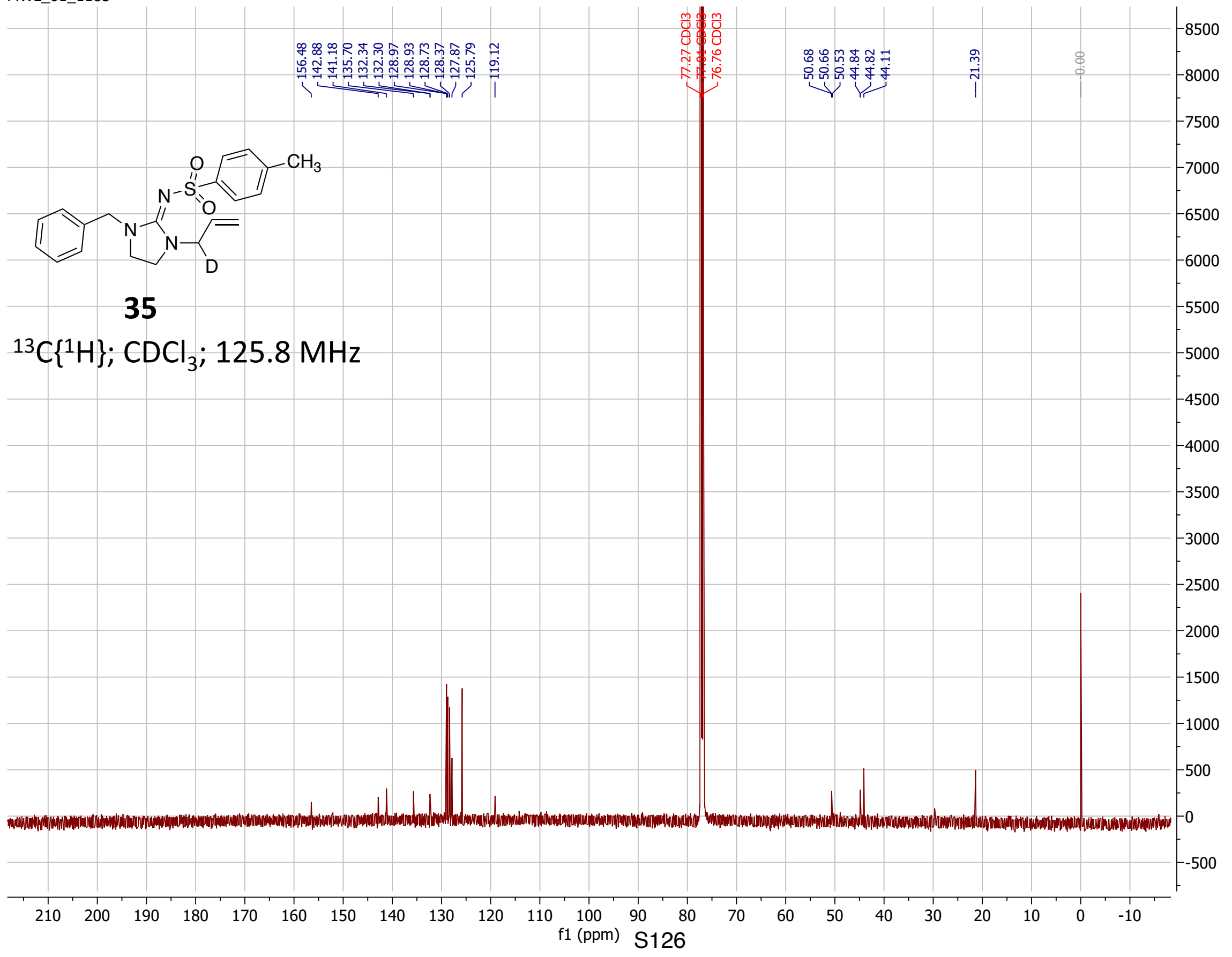




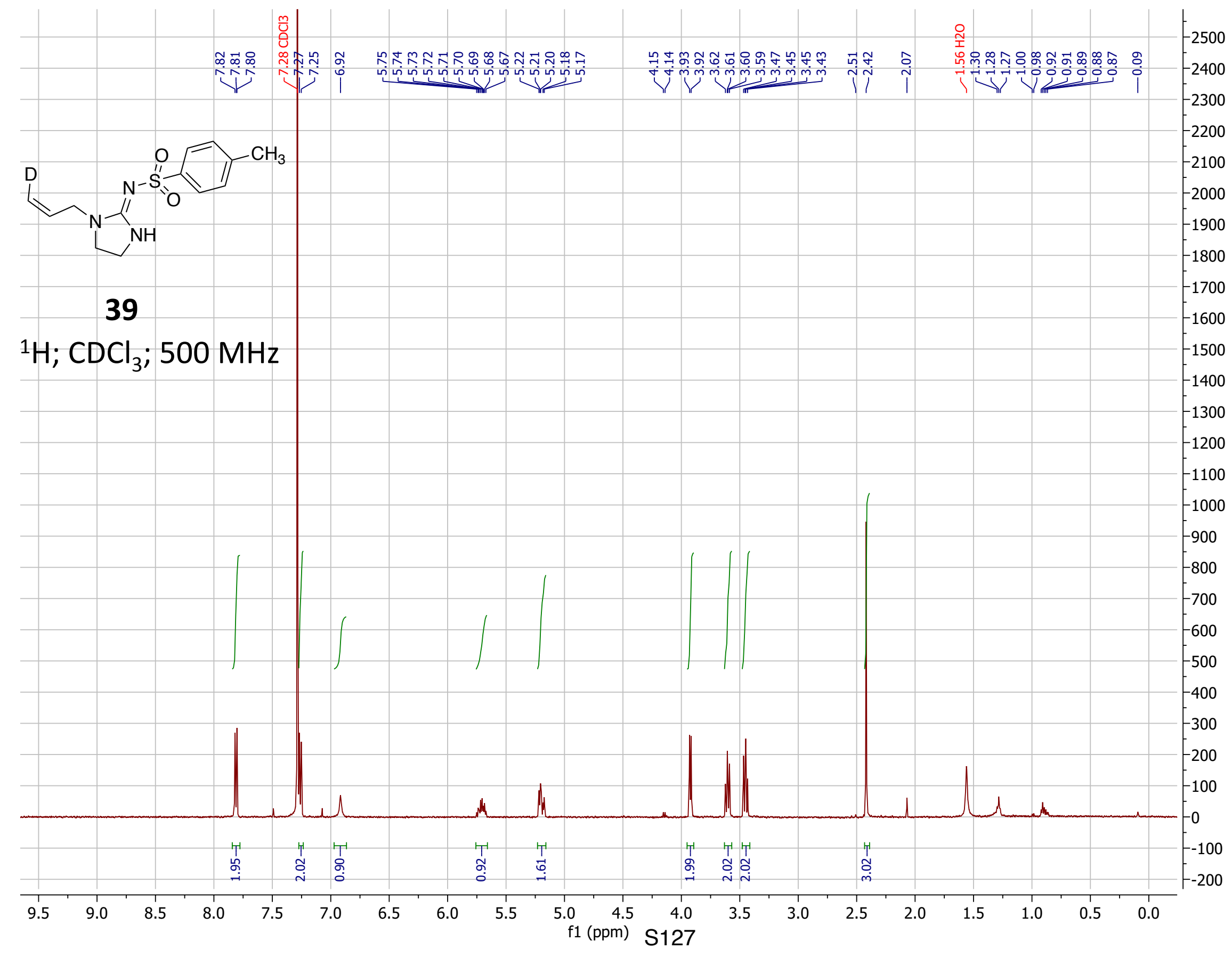




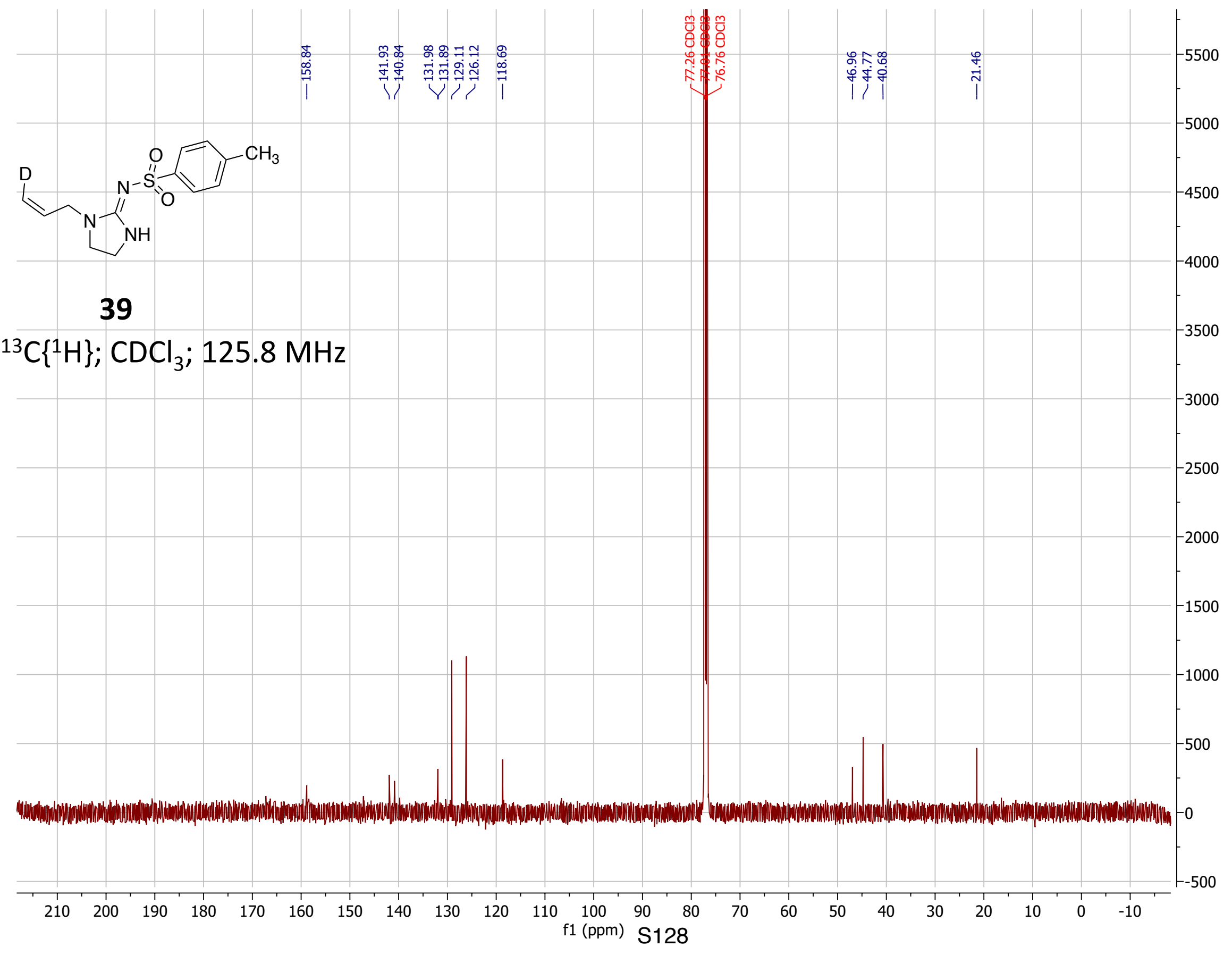

